\title{
Mathematical modeling of human brain physiological data
}

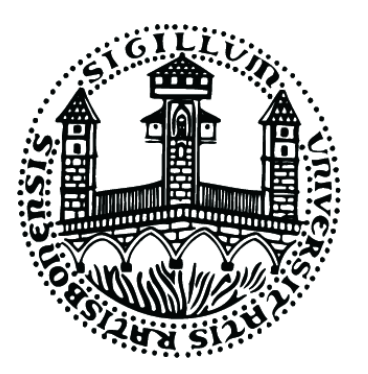

\section{DisserTATION}

zur Erlangung des Doktorgrades der Naturwissenschaften (Dr. rer. nat.) der Naturwissenschaftlichen Fakultät für Physik der Universität Regensburg

vorgelegt von

Matthias BöHм

aus

Regensburg 
Promotionsgesuch eingereicht am 22.12.2011.

Die Arbeit wurde durchgeführt am Institut für Biophysik und physikalische Biochemie unter der Anleitung von Prof. Dr. Elmar W. Lang.

Prüfungsausschuss:

Vorsitzender: $\quad$ Prof. Dr. Josef Zweck

1. Gutachter: $\quad$ Prof. Dr. Elmar W. Lang

2. Gutachter: Prof. Dr. Alexander Brawanski

3. Gutachter/Prüfer: Prof. Dr. Ingo Morgenstern 


\section{Contents}

1 Introduction 1

2 Anatomy of the human brain 5

2.1 Overview ...................... 5

2.2 Blood vessels . . . . . . . . . . . . . 6

2.2 .1 Arteries . . . . . . . . . . . . . . 6

2.2 .2 Capillaries . . . . . . . . . . . . . . . . . 10

2.2 .3 Veins . . . . . . . . . . . . . . . . 10

2.2 .4 Sinuses . . . . . . . . . . . . . . 10

2.3 Brain tissue . . . . . . . . . . . . . . . 11

2.4 Cerebrospinal fluid . . . . . . . . . . . . . . . . 12

3 Mathematical model $\quad 15$

3.1 First part: Cerebral Circulation . . . . . . . . . . . . 16

3.1 .1 Conservation of mass . . . . . . . . . . . . . 17

3.1 .2 Blood vessels . . . . . . . . . . . . . 18

3.1.3 Cerebrospinal fluid $(\mathrm{CSF}) \ldots \ldots \ldots \ldots$

3.1 .4 Brain tissue . . . . . . . . . . . . . . . . 21

3.1.5 Extra Compartment . . . . . . . . . . . . . 21

3.1.6 Set of differential equations . . . . . . . . . . . 22

3.2 Second Part: Oxygen Transport . . . . . . . . . . . . . . 22

3.2.1 Oxygen Dissociation Curve . . . . . . . . . . . 22

3.2 .2 Krogh cylinder . . . . . . . . . . . . . . . . 24

3.2.3 Theory of oxygen transport . . . . . . . . . 25

3.3 Connecting Part 1 and Part 2 . . . . . . . . . . 28

3.4 Extensions and modifications . . . . . . . . . . . . 29

3.4.1 Starling Resistor . . . . . . . . . . . . . . 29

3.4.2 Adjusting the Model . . . . . . . . . . . . . . 29

4 Default Values of the model 33

4.1 Fixed parameters . . . . . . . . . . . . . . . 34

4.1 .1 Volume ..................... 34

4.1 .2 Pressures . . . . . . . . . . . . . . . 34 
4.1 .3 Fluxes . . . . . . . . . . . . . . . . 34

4.1 .4 Constants . . . . . . . . . . . . . . 35

4.1.5 Capacitance . . . . . . . . . . . . . . 35

4.2 Calculated parameters . . . . . . . . . . . . 36

4.2 .1 Brain compartment . . . . . . . . . 36

4.2.2 CSF compartment . . . . . . . . . . . . 36

4.2 .3 Artery compartment . . . . . . . . . . . . 36

4.2.4 Capillary compartment . . . . . . . . . . . 37

4.2.5 Vein compartment . . . . . . . . . . . . 37

4.3 Boundary conditions for bHOM model . . . . . . . . . . 38

4.4 Adjustments for two hemispheres . . . . . . . . . . . . . 38

4.4 .1 Adjusted values . . . . . . . . . . . . . . . 38

4.4.2 Boundary conditions (two hemispheres) . . . . . . 39

4.5 Parameters for autoregulation . . . . . . . . . . . . . 40

4.5.1 Behavior of Autoregulation . . . . . . . . . . 40

4.6 Oxygen transport . . . . . . . . . . . . . . 45

4.6.1 Partial oxygen pressure in blood . . . . . . . . . 45

4.6.2 Properties of Krogh cylinder . . . . . . . . . . . . . . 46

4.6.3 Partial oxygen pressure in tissue . . . . . . . . . . 46

4.7 Summary . . . . . . . . . . . . . . . 46

5 General behavior of the bHOM model 47

5.1 Overview . . . . . . . . . . . . 47

5.2 No Swelling . . . . . . . . . . . . . . . . . . . . . 48

5.2.1 Case 1: no swelling, no autoregulation . . . . . . 48

5.2 .2 Case 2: no swelling, autoregulation . . . . . . . . 58

5.3 Swelling . . . . . . . . . . . . . . . . 69

5.3.1 Case 3: swelling, no autoregulation . . . . . . . . 69

5.3.2 Case 4: swelling, autoregulation . . . . . . . . . 75

5.4 Summary . . . . . . . . . . . . . . . . . 80

6 General behavior of the sHOM model 83

6.1 No swelling . . . . . . . . . . . . . . . 83

6.1.1 Case 1: no swelling, no autoregulation . . . . . . . . 83

6.1.2 Case 2: no swelling, autoregulation . . . . . . . . . 89

6.2 Swelling . . . . . . . . . . . . . . . . . 94

6.2.1 Case 3: swelling, no autoregulation . . . . . . . . . 94

6.2.2 Case 4: swelling, autoregulation . . . . . . . . . . 100

6.3 Summary . . . . . . . . . . . . . . . . 108

7 General behavior of esHOM model 109

7.1 Introductory remark . . . . . . . . . . . . . 110

7.2 No Swelling . . . . . . . . . . . . . . . . . . . . 110

7.2.1 Case 1: no swelling, no autoregulation . . . . . . . 110 
7.2.2 Case 2: no swelling, autoregulation . . . . . . . . . 114

7.3 Swelling . . . . . . . . . . . . . . . . . . 114

7.3.1 Case 3: swelling, no autoregulation . . . . . . . . 115

7.3.2 Case 4: swelling, autoregulation . . . . . . . . . . 120

7.4 Mixed autoregulation . . . . . . . . . . . . . . . 124

7.4.1 Case 5: no swelling, mixed autoregulation . . . . . 125

7.4.2 Case 6: swelling, mixed autoregulation . . . . . . . 133

7.5 Summary . . . . . . . . . . . . . . . . 140

8 Evaluation of the models 143

8.1 sHOM model . . . . . . . . . . . . . . . . . 144

8.1.1 Case 1: no swelling, no autoregulation . . . . . . . 144

8.1.2 Case 2: no swelling, autoregulation . . . . . . . . . 145

8.1.3 Case 3: swelling, no autoregulation . . . . . . . . . 145

8.1.4 Case 4: swelling, autoregulation . . . . . . . . . 147

8.2 esHOM model . . . . . . . . . . . . . . . . . . 147

8.2.1 Case 3: swelling, no autoregulation . . . . . . . . 148

8.2.2 Case 4: swelling, autoregulation . . . . . . . . . . 149

8.2.3 Case 5: no swelling, mixed autoregulation . . . . . . 150

8.2.4 Case 6: swelling, mixed autoregulation . . . . . . . 150

8.3 Placement of models . . . . . . . . . . . . . . . . . 151

8.3.1 Experimental data . . . . . . . . . . . . . . 152

8.3.2 Comparison with modeled data . . . . . . . . . . . 152

8.3 .3 Conclusion . . . . . . . . . . . . . . 159

9 Summary and outlook 161 


\section{Chapter 1}

\section{Introduction}

For the effective treatment of patients suffering from a severe brain damage it is indispensable to maintain an adequate oxygen supply to the patients' brain. To warrant this, several brain status parameters, as the temperature, the partial oxygen pressure in the tissue, the saturation of haemoglobin with oxygen and the pressure in the brain are continuously measured during treatment at the intensive care unit. Such a multimodal monitoring has been gaining more widespread use in daily practice, but is still under scientific examination, with respect to its effectiveness.

In the review "Does multimodality monitoring make a difference in neurocritical care?" [21], Sahuquillo et al. have examined the effectiveness of multimodal monitoring and pursued the question among clinicians, "whether or not neuromonitoring tools make a real difference in neurological outcome" [21], which is questioning the general usefulness of neuromonitoring itself.

Different analyses of the use of neuromonitoring, as were done by Shah et al. [24] and Cunningham et al. [4], showed, that the use of neuromonitoring itself does not significantly influence the progression of patients. In order to find the reason for this outcome, in [13] McIntosh et al. examined the cognitive processes of clinical staff in the interpretation of physiological multimodal monitoring data. Sahuquillo states, that according to this study, "senior doctors were able to make a correct diagnosis in only $68 \%$ cases, junior doctors in $58 \%$ and nurses in only $25 \%$ [13]" [21].

In [21] Sahuquillo gives a possible solution to the problem: namely, that "we need to use new computer-based methodologies and algorithms to analyses time-series data and extract useful information, independent of the skill of the enduser" [21], in order to extract useful information from multimodal monitoring for treating neurocritical patients.

From a physical point of view, the mathematical modeling of a complex system is one approach to gain insight into the dynamics of the system under different external conditions. To simplify the interpretation of the complex physiological processes, this mathematical model can be used, to reproduce 
the general behavior of the cerebral circulation and oxygen transport.

In this thesis, a basic model and two variations of this model will be presented. All these models have in common, that they consist of two parts. In the first part, the cerebral circulation, e.g. blood flow and cerebrospinal fluid flow is described by means of fundamental physiological assumptions. Here, a compartmental approach is used, in which compartments describe specific properties of arteries, capillaries, veins, brain tissue and cerebrospinal fluid. These compartments can interact with each other, where appropriate. In the second part, the oxygen transport from cerebral blood into the brain tissue is determined using the cerebral blood flow (CBF) as input.

First of all, a brief description of the anatomy of the human brain is given in chapter 2. The emphasis will be on specific key aspects of the human anatomy. These aspects will then be deployed in a mathematical model of the human brain in chapter 3 , which will allow to model the haemodynamical processes of the human brain and oxygen transport from the blood vessels into the tissue. The foundation of this work is based on a model, developed by Andreas Jung in cooperation with the Department of Neurosurgery of the University Hospital Regensburg. Due to some limitations of this model, two extensions will be proposed, which will improve the results gained with the original model substantially.

As the model will be used to simulate the haemodynamics of the human brain and oxygen transport into the tissue, a set of default parameters has to be determined, which will be done in chapter 4 .

After having provided a basis, an in-depth examination of the original model will be done in chapter 5 for four different scenarios, which cover certain areas of interest. In the first case, the simulations will be done with a disabled autoregulation mechanism, which will represent the easiest scenario from a model point of view. For the second case, the autoregulation mechanism will be enabled, a scenario, which represents the standard conditions of the human brain. As the model will be used to interpret neuromonitoring data of patients with a severe head injury, in the last two cases, the simulations will be done for the conditions, that a swelling occurs, with a disabled autoregulation mechanism for case 3 and an enabled autoregulation mechanism for case 4 . In this chapter, non- or not easily measurable values such as the compliances, resistances and volumes of the blood vessels, brain tissue and cerebrospinal fluid and the pressure inside each will be discussed.

Similarly, an extensive discussion will be done for the proposed extensions in chapter 6 and chapter 7 , with a focus on the differences between the models. In chapter 6 , the so called starling resistor will be used to simulate the resistance of the veins. Additionally to the use of the starling resistor, in chapter 7, a two hemisphere model will be used, contrary to the former approach. Therefore, an additional compartment for each type of blood vessel will be introduced. Also, this separation allows the discussion of two additional scenarios, in which the autoregulation mechanism is disabled in 
one hemisphere and enabled in the other and with and without a swelling.

In chapter 8 , the results of the extended models are re-examined from a medical point of view. Also, the alterations to the original model are justified, by using the simulations to reproduce and interpret a certain kind of behavior seen in measured data, recorded from patients with a server head injury. There, the partial oxygen pressure in tissue is anti-correlated to the mean arterial blood pressure and intracranial pressure. The cause of this behavior is not fully understood yet, but can be seen in more than $65 \%$ of the patient specific data sets [5]. It will be shown, that both proposed extensions, namely the use of the starling resistor for modeling the resistance of the veins and a two hemisphere approach, to the original model are necessary, to explain this kind of behavior from a model point of view in a qualitative and quantitative way.

In the last chapter, a summary of this work and an outlook to the future of this research will be given. 


\section{Chapter 2}

\section{Anatomy of the human brain}

In this chapter, a short introduction to the anatomy of the human brain will be given. From this foundation a model is derived in chapter 3, which will be used to simulate and analyze the haemodynamical processes and oxygen transport in the human brain.

The human brain itself is a complex and remarkable object. Although it accounts for less than $4 \%$ of an adult human body weight, it consumes about $15-17 \%$ of the total used oxygen of the human body [18].

In the next section an overview of the haemodynamical processes of the human brain, from an anatomical point of view, will be given. The following sections will give a more detailed description of certain aspects, that will be deployed in an abstract model of the human brain (see next chapter).

\section{$2.1 \quad$ Overview}

From a simplified point of view the haemodynamical processes in the brain can be summarized as follows: Oxygen saturated blood reaches the brain through the main arteries. There, the arteries split up into smaller arteries and arterioles, until they finally reach the capillary level. Arterioles are small arteries, which have the ability to actively change their diameter. In the capillaries, among other things, oxygen and carbon dioxide are exchanged with the tissue. Furthermore, this is the place where the production of the cerebrospinal fluid (CSF) takes place (see section 2.4). Then the capillaries reunite to venules, which are small veins but compared to veins stiffer, due to their smaller diameter, and veins. They ultimately end up into the big stiff veins called sinuses, from where venous blood flows back to the heart.

Mainly within the sagittal sinus the CSF will be absorbed. The arteries, arterioles, capillaries, venules and veins are surrounded by brain tissue, which itself is surrounded by the CSF. 


\subsection{Blood vessels}

The blood vessels itself can be divided into four classes: arteries/arterioles, capillaries, veins/venules and sinuses. Each class has different physiological properties and functions, which will be described in the next sections as needed for the model. From a model point of view arteries and arterioles can be treated as one type, although they have different properties (see section 2.2.1). The same can be done with venules and veins. Figure 2.1 shows a summary of the properties of all vessels except the sinuses.

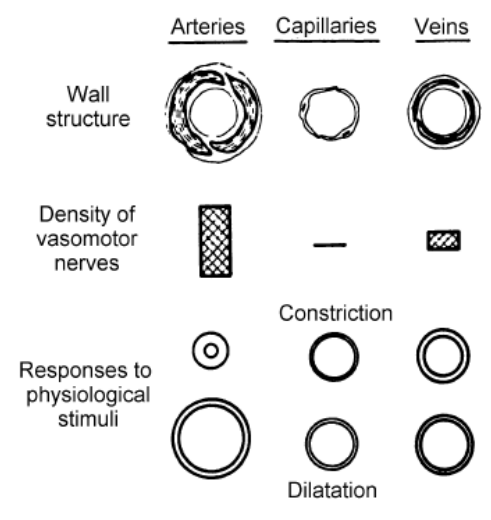

Figure 2.1: Scheme of the behavior of arteries, capillaries and veins related to their wall structure. The comparatively thick muscular layer and high density of vasomotor nerves account for considerable constriction and dilation ability of cerebral arteries. These responses are less pronounced in the veins (especially in the cerebral veins, which are devoid of a continuous muscles layer), and absent in the capillaries (original from [12], adapted from $[11])$.

\subsubsection{Arteries}

As mentioned before, the arteries supply the brain tissue among other things with oxygen and nutrition. There are four main arteries, namely the left and right internal carotid arteries and the left and right vertebral arteries, which are major arteries of the head and neck. All four main arteries originate directly from the aorta or branches from big vessels from the aorta and are located on the left and right side of the neck (compare fig. 2.2).

The two vertebral arteries merge into the basilar artery.* These three arteries are also called the vertebrobasilar system.

For the brain to function properly it is absolutely essential, that there is a steady supply of needed metabolites to the brain tissue, because the

${ }^{*}$ The technical term for this is called anastomosis. 


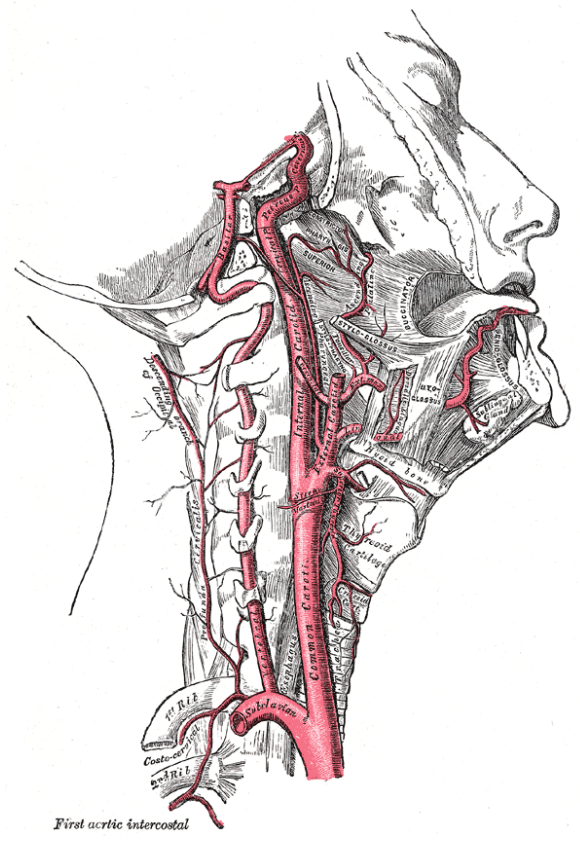

Figure 2.2: Shows the internal carotid artery (big blood vessel from bottom to top) and vertebral artery (smaller blood vessel from bottom to top) from the right side [6].

brain tissue cannot buffer oxygen (e.g., contrary to the muscle tissue). A steady supply is achieved by a regulation mechanism and an anatomical structure: the autoregulation of the arterioles and a network of connected and communicating arteries, called the circle of Willis.

\section{Autoregulation}

In medical literature autoregulation is defined as “... the capability of an organ to regulate its blood supply in accordance with its needs or as the intrinsic tendency of an organ to maintain constant blood flow despite changes in arterial perfusion pressure" [16].

As the arterioles have a muscular layer, contrary to the other classes of blood vessels (compare figure 2.1), they have the ability to actively change their diameter and therefore the blood flow.

A decrease in blood flow is achieved by constriction. As blood can be considered an incompressible fluid, by reducing the diameter of the arterioles an increase in the resistance of the blood vessels is achieved. Because the flux through the vessels depends on its resistance, the decrease of the diameter results in a decreased blood flow through the vessel. On the contrary, the blood flow can be increased, if the arterioles dilate, which results 
in a reduction of their resistance. Therefore, this regulation mechanism, which reacts on changes in blood flow and oxygen consumption, guarantees a steady supply over a wide range of the arterial blood pressure. The whole process to adjust the blood flow is called autoregulation.

It should be mentioned, that the change in volume is asymmetrical: The positive alteration in volume (dilation) is bigger than the negative alteration (constriction). As the change in diameter is limited, the range of the autoregulation is limited, too. If the maximal variation is obtained, there is no further possibility beyond that, to actively alter the blood flow.

In fig. 2.3 the cerebral blood flow (CBF) against the arterial blood pressure (ABP) of baboons is depicted (see [16]) with a working autoregulation mechanism.

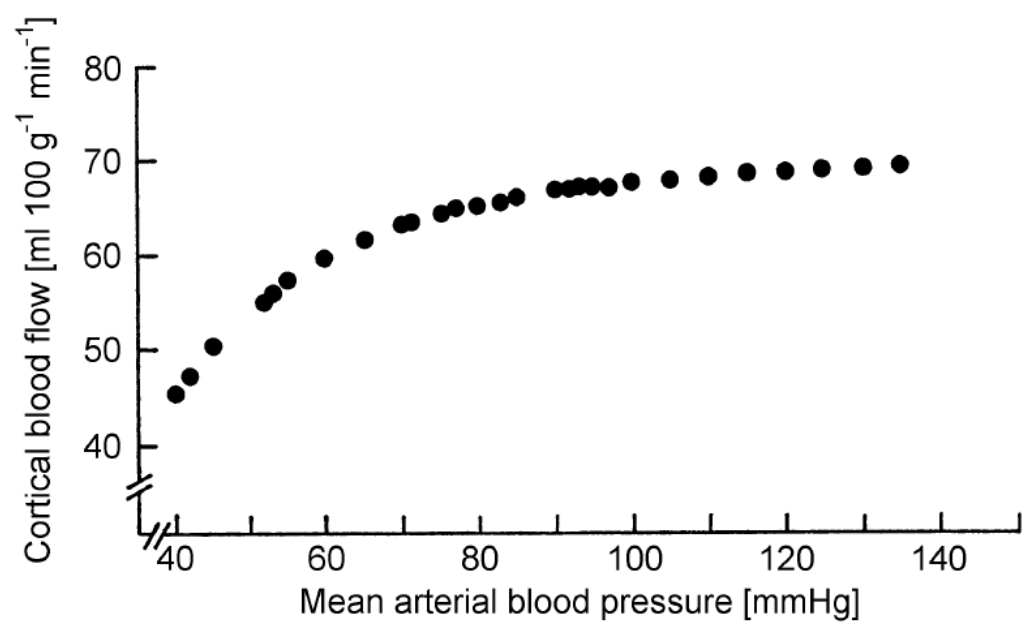

Figure 2.3: $\mathrm{CBF}$ against $\mathrm{ABP}$, measured on baboons [16].

As one can see, the blood flow is kept constant over a wide range of the ABP. In this experiment, for each point in the graph the ABP was fixed at a certain value and then the corresponding $\mathrm{CBF}$ was measured.

\section{Circle of Willis}

The circle of Willis (COW) is a ring of interconnected arteries, where most of the blood supply to the human brain stems from. Fig. 2.4 gives an anatomical view. It shows a view of the brain from below, with the front part of the brain located on the top. The circle of Willis itself is located approximately on the bottom side of the brain in the middle. A schematic plot of the COW is given in fig. 2.5, where the arteries forming the $\mathrm{COW}$ are located at the top of the picture. 


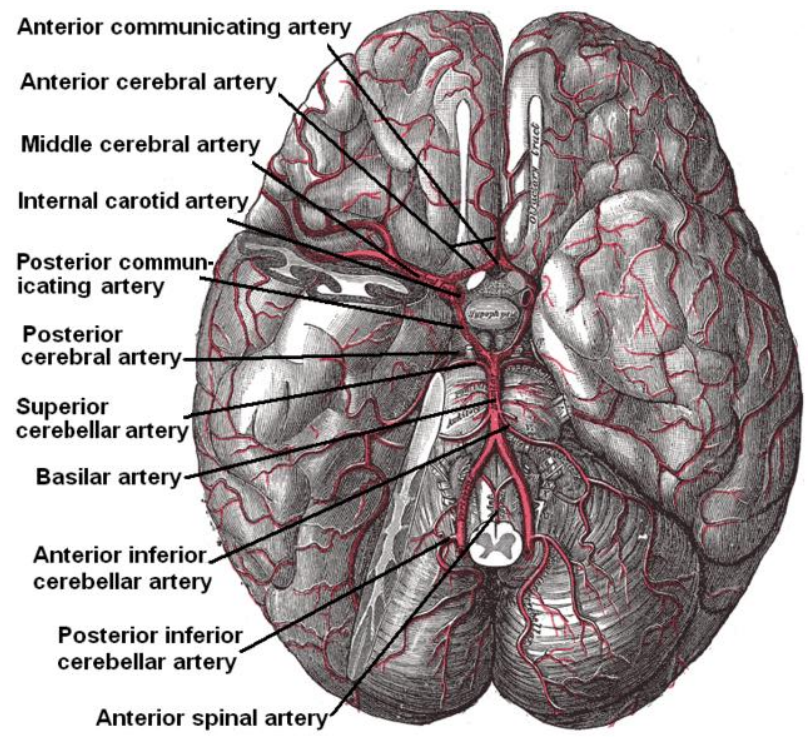

Figure 2.4: Anatomical view of the Circle of Willis from below [6].

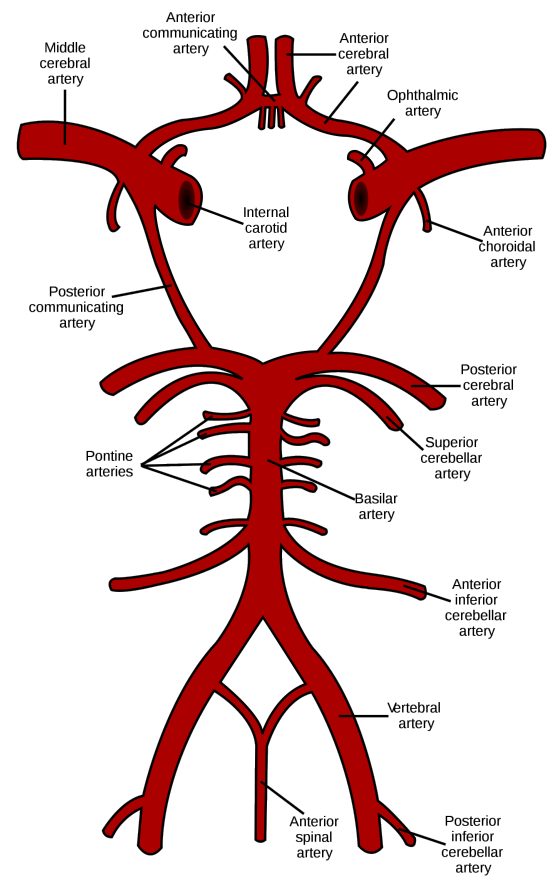

Figure 2.5: Schematic plot of the circle of Willis [31]; formed by the anterior and posterior communicating arteries, which create redundancies in the cerebral blood flow. 
The COW itself is formed by the anterior and posterior communicating arteries. The anterior communicating artery connects the left and right anterior arteries (at the center of the top of fig. 2.5), which are branches from the corresponding left and right internal carotid arteries (fig. 2.5, at the top on the left and right side). The posterior communicating arteries (fig. 2.5, below the internal carotid arteries) connect the posterior cerebral arteries and the middle cerebral arteries. The middle cerebral arteries are the continuation of the internal carotid arteries, whereas the posterior cerebral arteries are a branch of the basilar artery (fig. 2.5, center).

By the use of the communicating arteries redundancies in the cerebral blood flow are created. In the case of blocked or narrowed arteries the other arteries are able to preserve the cerebral perfusion and avoid ischemia, a shortage in blood supply.

\subsubsection{Capillaries}

Capillaries are branches from arterioles, and, contrary to arteries, thinwalled vessels (compare figure 2.1). They are the smallest type of blood vessel and therefore have the highest surface to diameter ratio compared to the other types of vessels. Due to these properties dissolved oxygen and nutrition can be transported to the tissue by diffusion. In return carbon dioxide and waste products are transported away from the tissue.

\subsubsection{Veins}

In contrast to the arteries veins are passive elements. Due to their size and structure veins behave like a rubber hose [11]. Depending on the difference between the blood pressure in the veins and the pressure in the brain tissue and CSF, respectively, their diameter can vary in size. If the pressure difference becomes negative, veins can collapse and therefore suspend cerebral blood flow in its corresponding surroundings.

\subsubsection{Sinuses}

The veins reunite into bigger veins, the sinuses. This network is also called the dural venous sinuses. It is located between the layers of the dura mater in the brain. Deoxygenated blood flows from internal veins and veins on the surface of the brain into the sinuses, which ultimately empty into the internal jugular veins. In addition, the cerebrospinal fluid (CSF) from the subarachnoid space, the interval between the arachnoid membrane and pia mater around the brain, is absorbed in the sinuses, mainly in the sagittal sinus superior (see section 2.4), which is the biggest sinus in the network. The sinuses have to be stiff, as the reabsorption process is pressure dependent and under normal conditions the pressure inside the sinuses is smaller than the CSF pressure. If the sinuses had the same physical properties as the 
veins, they would be compressed, because of the higher pressure of CSF . Similar to the veins this would result in a collapse of the sinuses.

\subsection{Brain tissue}

The human brain consists of a soft and compressible tissue and can be divided into a left and right hemisphere. The tissue can be distinguished into white and grey brain matter. Grey matter consists mainly of neural cell bodies, where most of the brain activity takes place. It is located mainly at the surface of the brain. The white matter consists mainly of so called axons, which connect various grey matter regions of the brain to each other and the grey matter to the rest of the body, respectively. They are the nerve tract. About $57 \%$ of the human brain is made up of grey matter whereas $43 \%$ is white matter [17]. However, the grey matter consumes more than $80 \%$ of the total oxygen used by the brain [18].

\section{Global pressure-volume curve}

From experimental data the ramifications of brain swelling are well known. There are two types of experiments [28]: Inflation of a balloon, to simulate a swelling, and injection of fluid from outside.

As for the balloon experiment, at the beginning, the extra volume occupied by the balloon can be compensated by a reduction of the CSF, so that the intracranial pressure (ICP) remains constant. When there is no CSF left, the ICP increases, due to a compression caused by the inflating of the balloon.

In the second type of experiment, a known amount of fluid is added or subtracted to the amount of CSF and its ramification on the CSF pressure is measured. Fig. 2.6 shows the outcome of such an experiment, the global pressure-volume curve (compare [26]).

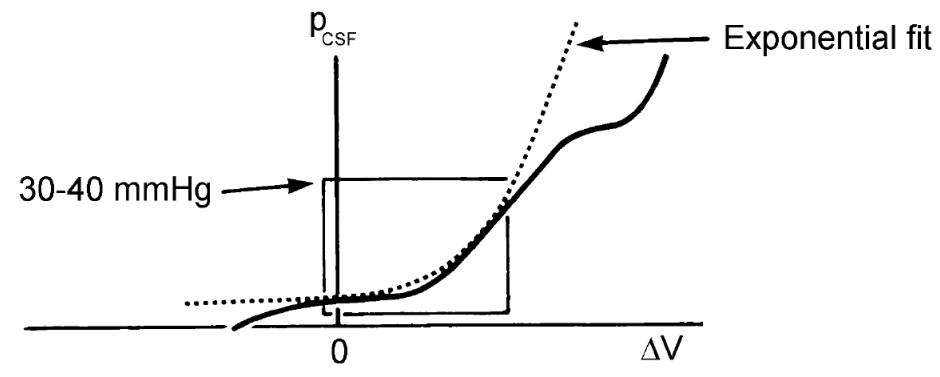

Figure 2.6: Measured global pressure-volume curve (adapted from [26]). 


\subsection{Cerebrospinal fluid}

The brain tissue is surrounded by the so called cerebrospinal fluid (CSF). It is able to absorb and distribute forces, that act from inside (e.g., tumor growth, swelling) and outside (accidental impact) on the brain.

CSF is generated by a network of blood vessels, called plexus choroidei (compare fig. 2.7, left). These reside in four cavities inside the human head: the ventricles.
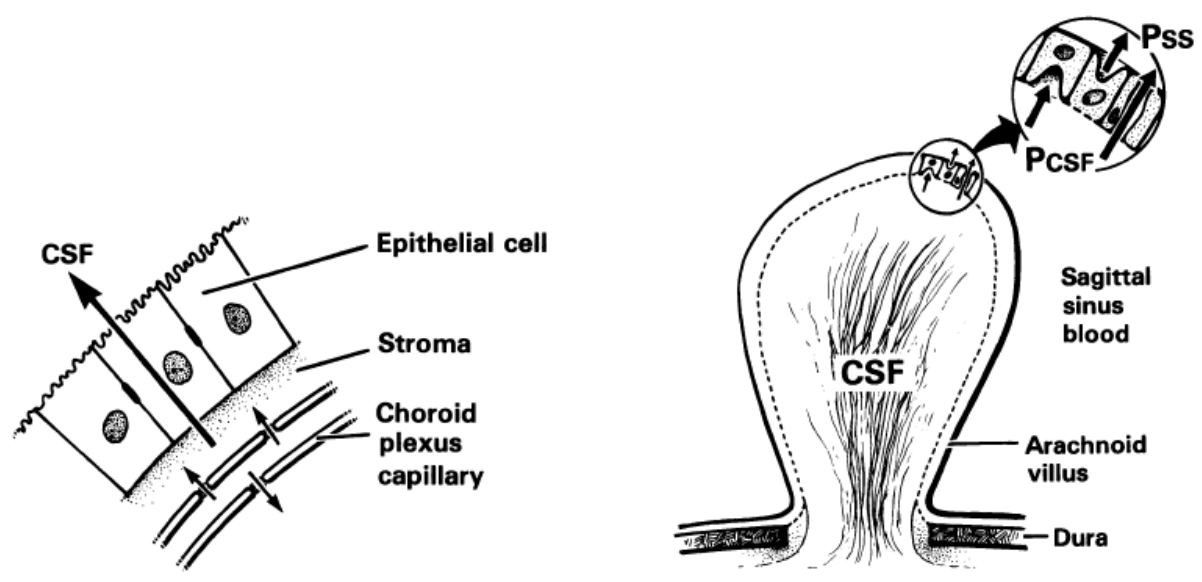

Figure 2.7: Cerebrospinal fluid is produced in the choroid plexus capillaries in the ventricles (left); the driving force is the pressure difference between the blood pressure at the capillary level and the CSF compartment. Cerebrospinal fluid absorption takes place mainly in the sagittal sinus (right) and depends on the pressure difference between the CSF pressure and the pressure in the sagittal sinus (figures from [26]).

There are four ventricles, namely the left and right side ventricle, and the $3 \mathrm{rd}$ and 4 th ventricle. The network is most potent in the side ventricles, where the bigger part of the CSF is produced. From the side ventricles CSF flows through the foramina interventricularia in the third ventricle, where it gains additional influx. From there it reaches the fourth ventricle and finally leaves this cavity to the subarachnoid space. Most of the CSF flows upward to the sinus sagitalis superior, a small part flows downward to the spinal cord. Reabsorption of the CSF occurs in the granulationes arachnoideales (compare figure 2.7, right) at the pia mater. The largest granulations reside in the sagittal sinus superior, which is shown in figure 2.8 , but are present in other sinuses, too. Figure 2.9 shows the whole CSF circulation.

The arachnoid granulations themselves act as one-way valves. Under normal conditions, the CSF pressure is higher than the pressure in the sagittal 


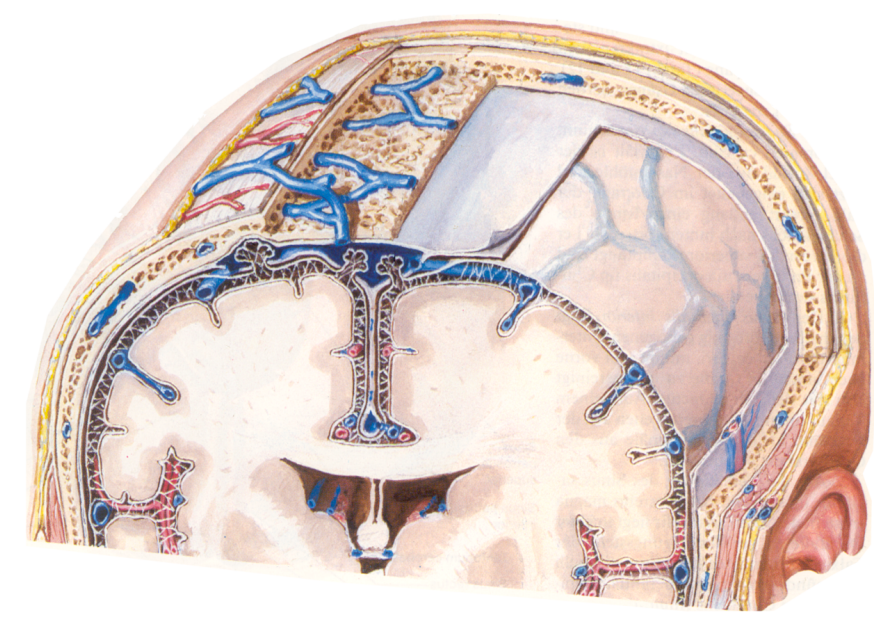

Figure 2.8: Frontal view of the cerebrospinal fluid absorption in the sagittal sinus (big venous blood vessel at the top of the brain; (adapted from [15]).

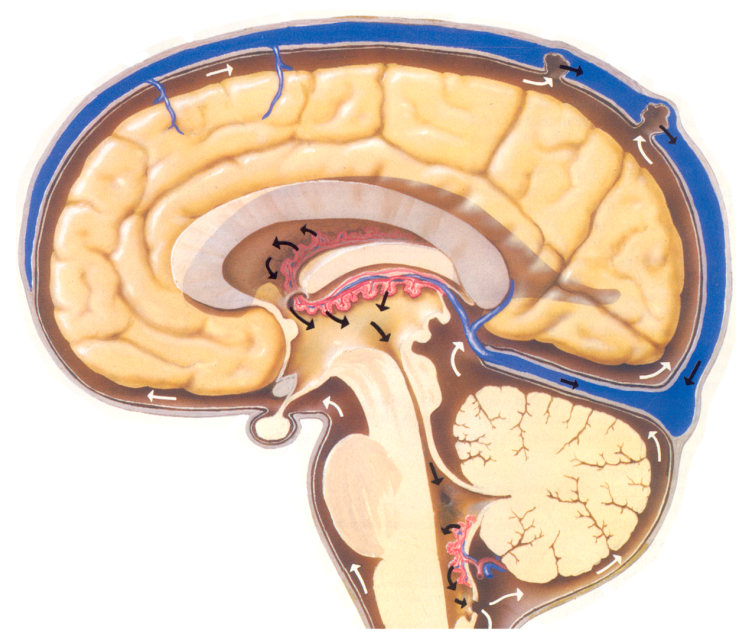

Figure 2.9: Cerebrospinal fluid circulation; CSF is produced in the ventricles (center of the brain). Most of the produced CSF flows upwards and is absorbed mainly in the sagittal sinus (big venous blood vessel at the top of the brain). The rest flows downwards the spinal cord (adapted from [15]). 
sinus. Therefore, CSF flows through the granulations into the blood. But if the CSF pressure is lower, fluid cannot flow back from the blood.

The amount of CSF of an adult is about 125-150 ml. Approximately 430-450 $\mathrm{ml}$ per day of CSF is produced and absorbed, so that about every 6-7 hours the complete CSF is exchanged (compare [15]).

By a change in volume the CSF regulates the capacity of the human skull, e.g., when a swelling inside the head occurs. With an increase of the pressure inside the skull, the pressure of the CSF increases, too. As the CSF production depends on the difference between the pressure in the plexus choroidei and the pressure in the CSF, this results in a lower CSF production. On the contrary, the CSF absorption increases due to an increase in the difference between CSF pressure and the pressure inside the sinuses, which altogether results in a decrease of CSF.

Experiments show, that from the total amount of CSF only $30 \mathrm{ml}$ can be used to adjust changes in cerebral volume, as stated in [11]. 


\section{Chapter 3}

\section{Mathematical model of the human brain}

For the effective treatment of patients suffering from a severe brain damage, it is indispensable to maintain an adequate oxygen supply to the patients' brain. To warrant this, several brain status parameters, such as the temperature, the partial oxygen pressure in the tissue, the saturation of haemoglobin with oxygen and the pressure in the brain are continuously recorded at the intensive care unit.

However, the interpretation of the recorded data set is not an easy task, due to the complex underlying physiological processes and their influence on each other. Therefore, it was necessary to develop a model, which is able to reproduce the general behavior of the cerebral circulation and oxygen transport, in order to gain a better understanding of the underlying processes and their connections.

The model used in this work is based on the work done by Andreas Jung in collaboration with the Department of Neurosurgery of the University Hospital Regensburg [11]. In the next sections a description of this basic model is given.

The model consists of two parts. In the first part, the cerebral circulation, e.g. blood flow and cerebrospinal fluid flow are modeled by means of simple physiological assumptions. In this case a compartmental approach is used. Its foundation is mainly based on the approach used/proposed by Ursino [29]. In the second part the oxygen transport from cerebral blood into the brain tissue is determined using the cerebral blood flow $(\mathrm{CBF})$ as an input.

To overcome some of its limitations in reproducing measured data, a couple of extensions and modifications to the model will be proposed in the last section. Additionally, these extensions will better reflect the natural conditions of human brain physiology.

To better distinguish the models among each other by name, the follow- 
ing naming convention will be used throughout this work: As all models are used to simulate the haemodynamical process inside the human brain and the oxygen transport, for all models the abbreviation HOM will be used. Here, HOM stands for "Haemodynamics and Oxygen transport Model". As the model used in [11] lays the basis for the extensions, it will be prefixed with a "b", which stands for "basic". If the starling resistor is used to model the resistance of the veins, a " $\mathrm{s}$ " is used as prefix. When a two hemisphere approach is used, the whole abbreviation will be prefixed with an "e", which stands for "extended". For example, if both extensions are used, the model will be called esHOM.

\subsection{First part: Cerebral Circulation}

To simulate cerebral circulation, one possible approach is to divide the fluid and matter constituents within the human head into several interacting subunits, so called compartments. Fig. 3.1 shows the layout of the model.

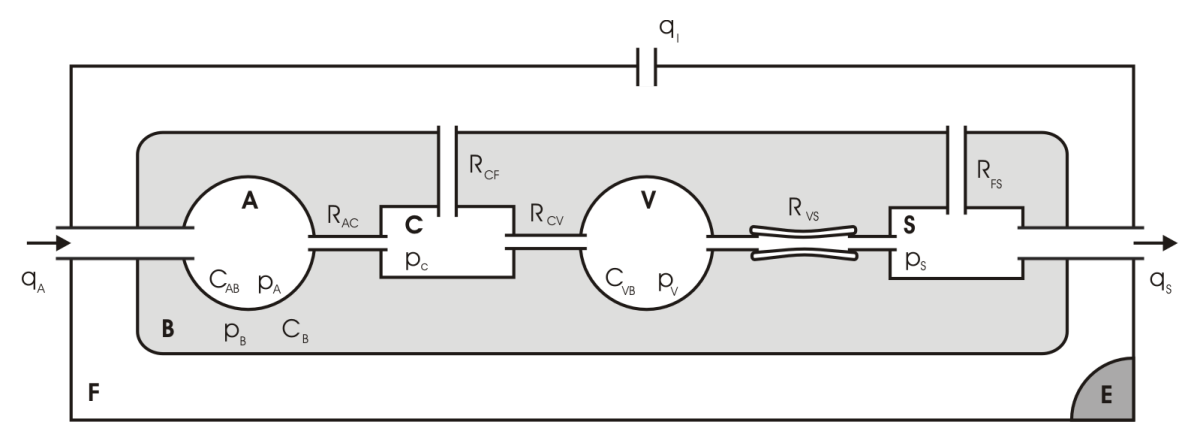

Figure 3.1: Abstract model for simulating haemodynamical processes inside the human brain; consists of compartments: A - arteries (combined with arterioles), C - capillaries, V - veins (combined with venules), S - sagittal sinus, B - brain tissue, F - cerebrospinal fluid, E - extra volume describing brain swelling. The in- and outflows of the system are given by $q_{X}$, the resistance between two compartments $X$ and $Y$ by $R_{X Y}$. The pressure inside a compartment $X$ is determined by $p_{X}$. Compartments, which can vary in size, are described by a compliance $C_{X(Y)}$.

Oxygen saturated blood reaches the brain through the main arteries (inflow of the model). There, the arteries split up into smaller arteries and arterioles (described by compartment A), until they finally reach the capillary level (described by compartment C). In the capillaries, among other metabolites, oxygen and carbon dioxide are exchanged with the tissue. Furthermore, this is the place where the production of the cerebrospinal fluid (CSF) takes place (internal inflow of compartment $\mathrm{F}$ ). Then, the capillaries 
reunite to venules and veins (compartment V) and ultimately end up into the big stiff veins called sinuses (compartment $S$ ), from where venous blood flows back to the heart (outflow of the model). Within the sagittal sinus the CSF will be absorbed (outflow of compartment F). The arterial system, capillaries, venous system and sinuses are surrounded by brain tissue (compartment B), which itself is surrounded by the CSF. All compartments are enclosed by the cranial bone, which therefore acts as closed compartment. For experimental reasons an extra compartment $\mathrm{E}$ is introduced, which allows the simulation of brain swelling. Additionally, it is possible to simulate the injection of fluid from outside with $q_{I}$ (pressure/volume experiments).

\subsubsection{Conservation of mass}

As a starting point for the modeling of the compartments the conservation of mass is used:

$$
\sum q_{i}=\frac{d m}{d t}=\underbrace{\frac{\partial m}{\partial \rho}}_{=V} \frac{\partial \rho}{\partial t}+\underbrace{\frac{\partial m}{\partial V}}_{=\rho} \frac{\partial V}{\partial t}
$$

whereas $m(t)=\rho(t) V(t)$. It is obvious, that a change in mass, and therefore a change in the density or/and volume of a compartment, can only occur, if the sum over all inflows and outflows $q_{i}$ is not zero.

For eq. 3.1 the following physiological assumptions have to be made:

- Blood and CSF are incompressible media ( $\rho=$ const).

- Brain tissue is a compressible medium $(\rho \neq$ const $)$.

- Flow in blood vessels is laminar; the Hagen-Poiseuille law is used to describe the flow $q_{X Y}$ between compartments $X$ and $Y$ :

$$
q_{X Y}=\frac{\Delta p_{X Y}}{R_{X Y}}=\frac{p_{X}-p_{Y}}{R_{X Y}}
$$

- Membranes between compartments are assumed to be elastic. The volume of the compartment can be calculated by

$$
V-V_{0}=\int_{0}^{p} C(\varphi) d \varphi \text { with } p=p_{\text {ext }}-p_{\text {int }}
$$

where the compliance $C(\varphi)$ describes a volume change per unit of pressure change across an elastic structure.

- The cranial bone acts as a closed compartment; the total volume is therefore constant (Monroe-Kellie doctrine [16]). Consequently, the change in total volume of all compartments equals zero:

$$
\sum_{i} \dot{V}_{i}=0
$$


Taking into account the different properties of each compartment, a nonlinear system of differential equations describing cerebral circulation including haemodynamics can be derived.

\subsubsection{Blood vessels}

For modeling purposes the blood vessels can be separated into four different types, namely arteries (and arterioles), capillaries, veins (and venules) and sinuses, whereas each type has its own properties.

They all have in common, that their resistance can be modeled by using the Hagen-Poiseuille law. The resistance $R_{X Y}$ is therefore inversely proportional to $r^{4}$ :

$$
R_{X Y}=\frac{k_{R}^{\prime}}{r^{4}}=\frac{k_{R}}{V^{2}}
$$

whereas $k_{R}$ is a constant. All blood vessels can be assumed to be straight pipes with radius $r$. Therefore, their volume $V$ is proportional to $r^{2}$.

\section{Arteries}

Arteries and arterioles, respectively, are active elements and are responsible for the so called autoregulation, a mechanism to maintain a constant cerebral blood flow over a wide range of blood pressure. This effect is achieved by the arteries/arterioles ability to dilate and constrict, which results in a variable resistance:

$$
R_{A C}=\frac{k_{R_{A C}}^{\prime}}{r^{4}}=\frac{k_{R_{A C}}}{V_{A}^{2}}=\frac{k_{R_{A C}}}{C_{A B}^{2}\left(p_{A}-p_{B}\right)^{2}}
$$

whereas $k_{R_{A C}}=$ const and $V_{A}=C_{A B}\left(p_{A}-p_{B}\right)$. The compliance $C_{A B}$ can be described by:

$$
\frac{d C_{A B}}{d t}=-\frac{1}{\tau}\left(C_{A B}-C_{A B r e g}(\alpha x)\right) \text { with } x=\frac{q_{A C}-q_{A C_{n}}}{q_{A C_{n}}}
$$

whereas $q_{A C}$ equals the cerebral blood flow and $q_{A C_{n}}=$ const describes the $\mathrm{CBF}$ required for the standard tissue metabolism. The degree of autoregulation is given by $\alpha$, and $\tau$ is the time constant of the regulation. The regulation term $C_{A B r e g}$ itself has the following form, which is proposed by Ursino and Lodi in [29]:

$$
C_{A B r e g}(\alpha x)=C_{A B n}-\Delta C_{A B} \cdot \tanh \left(\frac{\alpha x}{\Delta C_{A B}}\right)
$$

Thereby $\Delta C_{A B}$ represents the maximum gain for the autoregulation and $C_{A B n}$ is the compliance of the arteries for the standard CBF. As the autoregulation curve is not symmetrical, $\Delta C_{A B}$ assumes different values for 
dilation $(x<0)$ and constriction $(x>0)$ :

$$
\Delta C_{A B}= \begin{cases}\Delta C_{A B 1} & x<0 \\ \Delta C_{A B 2} & x>0\end{cases}
$$

Figure 3.2 shows the function $\tanh (\alpha x)$ for different $\alpha$. With raising difference between the $\mathrm{CBF}$ and the standard $\mathrm{CBF}$ the contribution of $C_{A B r e g}$ to the change in $C_{A B}$ increases, until it reaches an extremum, which is determined by $\Delta C_{A B}$. With increasing $\alpha$ this extremum is reached faster.

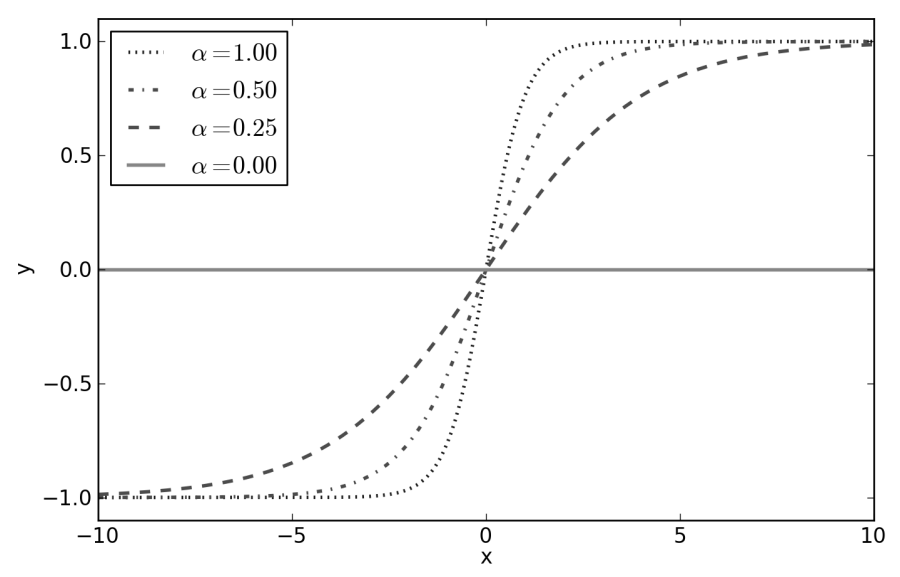

Figure 3.2: $\tanh (\alpha x)$ for different $\alpha$; with increasing $\alpha$ the slope around $x=0$ gets steeper.

\section{Capillaries}

Capillaries are passive elements. For the simplification of the model it is assumed, that they do not vary in size and therefore have a constant resistance:

$$
R_{C V}=\frac{k_{R_{C V}}}{V_{C}^{2}}=\text { const }
$$

It should be noted, that the production of CSF takes place at the capillary level (compare section 2.4), which will be described in section 3.1.3.

\section{Veins}

In contrast to the arteries, the veins are passive elements. Here, the venous system can be separated into two parts: 
1. The first part is leading from the small capillaries to the bigger veins; the resistance is assumed to be constant (small blood vessels named venules; contained in the capillary part of the model).

2. The second part is leading from the bigger veins to the sinuses; here, the veins behave like a rubber hose (no active elements). The volume depends only on the difference between venous blood pressure and the pressure surrounding the veins (intracranial pressure).

In order to determine the resistance of the veins itself

$$
R_{V S}=\frac{k_{R_{V S}}}{V_{V}^{2}}
$$

the volume of the veins has to be determined and therefore their compliance. In literature there are two methods to determine the volume of the veins. One approach is proposed by Ursino in [28]:

$$
C_{V B}=\frac{1}{k_{V}\left(\sqrt{\left(p_{V}-p_{B}\right)^{2}}+p_{V_{0}}\right)}
$$

Eq. 3.12 can then be used to calculate the change in volume of the veins $\dot{V}_{V}$, as done in [28]. The other approach is used in [11], where $\dot{V}_{V}$ is calculated by eq. 3.1 and using the assumption, that the density $\rho=1$ :

$$
\frac{d}{d t} V_{V}=q_{C V}-q_{V S}
$$

The second method will be used in this work.

\section{Sagittal Sinus}

The sinuses are stiff big veins, in which all blood is transported back to the heart and lung, to be accumulated again with oxygen and nutrition. Additionally, CSF is absorbed in the sinus (see section 3.1.3). In the original model, ${ }^{*}$ a constant outflow $q_{S}$ is assumed. This assumption is supported by short time range experimental measurements, which show a low variation in blood flow inside the sinus [28]. As the sinuses are stiff, their resistance does not change. Therefore, the pressure can be assumed to be constant, too:

$$
p_{S}=\text { const }
$$

\subsubsection{Cerebrospinal fluid (CSF)}

The brain tissue is surrounded by the so called cerebrospinal fluid. As mentioned before this fluid is produced at a capillary level and (mainly) absorbed

* proposed by Ursino and adapted by Jung 
at the sagittal sinus. The driving forces of production and absorption are the pressure differences between capillaries and the CSF compartment, and between the CSF compartment and the sagittal sinus, respectively:

$$
q_{C F}=\frac{p_{C}-p_{B}}{R_{C F}} \text { and } q_{F S}=\frac{p_{B}-p_{S}}{R_{F S}}
$$

It should be mentioned, that a reversal of both fluxes is not possible. Therefore, their resistance can be modeled by:

$$
\begin{aligned}
R_{C F}\left(p_{C}-p_{B}\right) & = \begin{cases}R_{C F n} & \text { if }\left(p_{C}-p_{B}\right)>0 \\
\infty & \text { else }\end{cases} \\
R_{F S}\left(p_{B}-p_{S}, V_{F}\right) & = \begin{cases}R_{F S n} & \text { if }\left(p_{B}-p_{S}\right)>0 \text { and } V_{F}>0 \\
\infty & \text { else }\end{cases}
\end{aligned}
$$

Also the amount of CSF volume must additionally be taken into account, as the situation can arise, that no fluid is available for absorption.

\subsubsection{Brain tissue}

The brain tissue consists of soft material, which means, it is a compressible medium. Because the membrane between brain tissue and the CSF compartment is very thin, the pressure difference between both compartments $\left(p_{B}-p_{F}\right)$ can be presumed to be zero. Experiments support this assumption (compare [32]).

For the model a modified version of the formula proposed by Ursino in $[29]$ is used for the compliance:

$$
C_{B}\left(p_{B}\right)=\frac{1}{k_{B}\left(\left|p_{B}\right|+p_{B_{0}}\right)}
$$

To overcome a divergence at $p_{B}=0$ an extra pressure $p_{B_{0}}$ is added, as proposed by [11].

\subsubsection{Extra Compartment}

To be able to simulate a swelling of the brain, two extra flows are introduced. These correspond to animal experiments, which are done in two ways [28]:

1. Inflation of a balloon; simulated with compartment E. The volume changes are defined by $q_{E}$.

2. Injection of fluid from outside; described by $q_{I}$.

With these experiments a brain swelling can be simulated in a controllable manner. 


\subsubsection{Set of differential equations}

Putting this altogether, one gets the following set of equations:

$$
\begin{aligned}
A: & q_{A}-q_{A C}=\dot{C}_{A B} \Delta p_{A B}+C_{A B} \Delta \dot{p}_{A B} \\
C: & q_{A C}-q_{C B}-q_{C V}=0 \\
V: & q_{C V}-q_{V S}=C_{V B} \Delta \dot{p}_{V B} \\
M K: & q_{I}+q_{E}+q_{C B}-q_{B S}= \\
& \quad C_{B} \dot{p}_{B}-\dot{C}_{A B} \Delta p_{A B}-C_{A B} \Delta \dot{p}_{A B}-C_{V B} \Delta \dot{p}_{V B} \\
S: & q_{V S}-q_{S}+q_{B S}=0
\end{aligned}
$$

with $R_{V S}=R_{V S}\left(V_{V}\right)$ and $R_{F S}=R_{F S}\left(p_{B}-p_{S}, V_{F}\right)$, and

$$
\begin{gathered}
\frac{d}{d t} V_{V}=q_{C V}-q_{V S}, \frac{d}{d t} V_{F}=q_{I}+q_{C B}-q_{B S} \\
\frac{d C_{A B}}{d t}=-\frac{1}{\tau}\left(C_{A B}-C_{A B r e g}(\alpha x)\right) \text { with } x=\frac{q_{A C}-q_{A C n}}{q_{A C n}}
\end{gathered}
$$

This set of equations is then solved numerically under the assumption of a constant outflow.

\subsection{Second Part: Oxygen Transport}

The second part of the model calculates the oxygen transport from cerebral blood to brain tissue. Blood flows through the lungs, where due to diffusion processes the blood is saturated with oxygen. Under normal conditions, $98 \%$ of oxygen molecules are bound to haemoglobin. Only $2 \%$ are dissolved in blood, whereas only dissolved oxygen can diffuse into brain matter [8]. The total amount of oxygen in blood $\mathrm{CbO}_{2}$ is then described by the following sum:

$$
\mathrm{CbO}_{2}=\underbrace{\beta \cdot \mathrm{SbO}_{2}}_{\approx 98 \%}+\underbrace{\gamma \cdot \mathrm{pbO}_{2}}_{\approx 2 \%}
$$

where $\beta=0.201 \frac{\mathrm{cm}^{3} \mathrm{O}_{2}}{\mathrm{~cm}^{3}}$ and $\gamma=3 \cdot 10^{-5} \frac{\mathrm{cm}^{3} \mathrm{O}_{2}}{\mathrm{~cm}^{3} \mathrm{mmHg}}$ (compare [22]). By $\mathrm{SbO}_{2}$ the saturation of blood with oxygen is given, whereas $\mathrm{pbO}_{2}$ describes the partial oxygen pressure in blood.

\subsubsection{Oxygen Dissociation Curve}

In general, the ratio between bounded and dissolved oxygen is described by the so called oxygen dissociation curve (ODC). The ODC depends on the $\mathrm{CO}_{2}$ content of the blood, as can be seen in fig. 3.3. As the curve has a steep slope between 10 and $50 \mathrm{mmHg}$, large variations in the saturation 


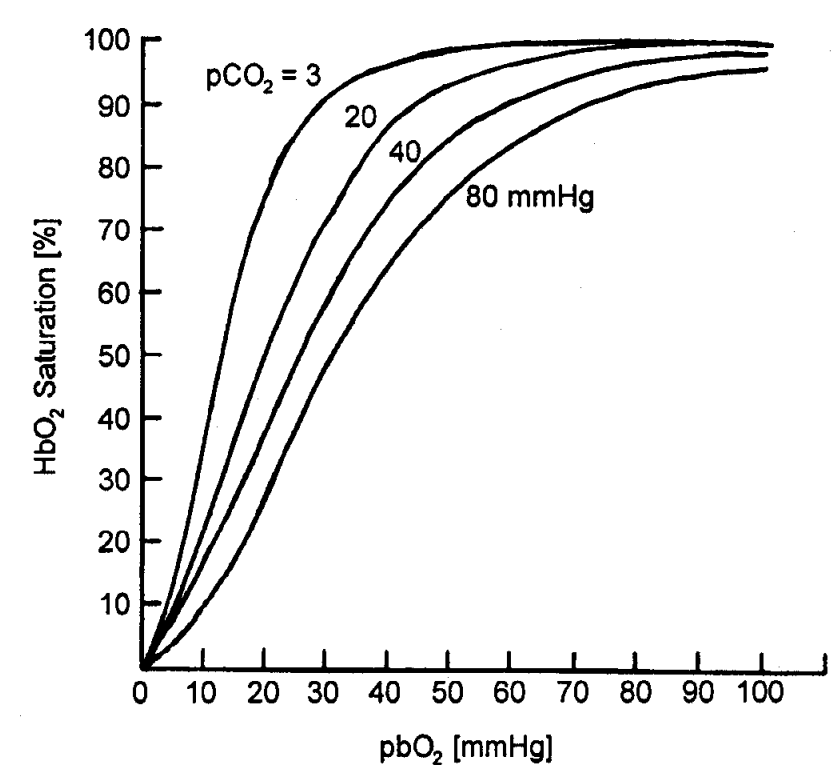

Figure 3.3: Oxygen dissociation curve; the diagram show the connection between the dissolved oxygen in the blood and the the partial pressure of oxygen $\left(\mathrm{pbO}_{2}\right)$ in blood, respectively, and the saturation of haemoglobin with oxygen $\left(\mathrm{HbO}_{2}\right)$. The curve depends on the partial carbon dioxide pressure $\left(\mathrm{pCO}_{2}\right)$, in which $\mathrm{pCO}_{2}=40 \mathrm{mmHg}$ depicts the standard value under normal conditions (adapted from [19]).

of haemoglobin with oxygen cause only small variations in partial oxygen pressure $\left(\mathrm{pbO}_{2}\right)$. Therefore, haemoglobin acts as a buffer for oxygen.

To model the saturation of haemoglobin with oxygen, here, a simple approximation for the ODC is used. Proposed by Hill in [9], the following variant of this equation, given in [27], is used:

$$
\mathrm{HbO}_{2} \text { saturation }=\mathrm{SbO}_{2}=\frac{\left(\frac{p}{p_{50}}\right)^{n}}{1+\left(\frac{p}{p_{50}}\right)^{n}}
$$

whereas $p$ is the partial pressure of oxygen in blood. The exponent $n$ and $p_{50}$ are constant values, whereupon $p_{50}$ is defined by the oxygen pressure in blood for a saturation of $50 \%$. The ODC dependence on $\mathrm{CO}_{2}$ is modeled with $n$. Both values can be determined experimentally (see section 4.6.1).

As mentioned before, for the oxygen supply to the tissue only dissolved oxygen in blood has to be taken into account. Because the surface/volume ratio of capillaries is much higher than for other vessels, oxygen supply of brain matter happens mainly at the capillary level. 


\subsubsection{Krogh cylinder}

The oxygen supply of brain tissue can be described by the so called Krogh cylinder [18], which is shown in fig. 3.4. The Krogh cylinder consist of

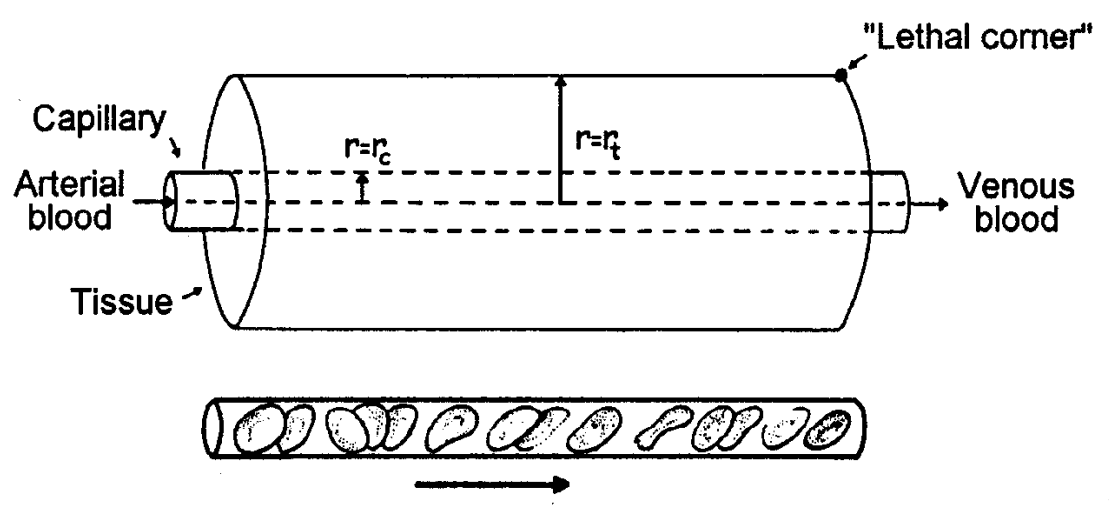

Figure 3.4: Krogh cylinder; capillaries with radius $r_{c}$ are surrounded by a tissue cylinder with radius $r_{t}$. Oxygenated blood cells flow through the capillaries and after dissociation into the blood, oxygen diffuses into the tissue. The point "Lethal corner" marks the point in the Krogh cylinder with the lowest oxygen content (adapted from [18]).

a capillary with radius $r_{c}$, which is surrounded by a tissue cylinder with radius $r_{t}$. It is assumed, that the oxygen pressure gradient between blood and tissue serves as the driving force of the diffusion process. For simplicity, only radial diffusion is taken into account. ${ }^{\dagger}$

For the capillaries the following additional assumptions for the model are made, which are not true in real life:

- Capillaries are straight pipes, running in parallel and having a unidirectional blood flow

- Capillaries are arranged in a hexagonal lattice (see fig. 3.5)

- Diameter is assumed to be constant and velocity profile of blood is uniform

- Blood flow is constant over the whole capillary length

- Capillary wall represents no resistance to the oxygen diffusion

- Oxygen pressure in capillary is assumed to be constant in $r$ and $\varphi$ 


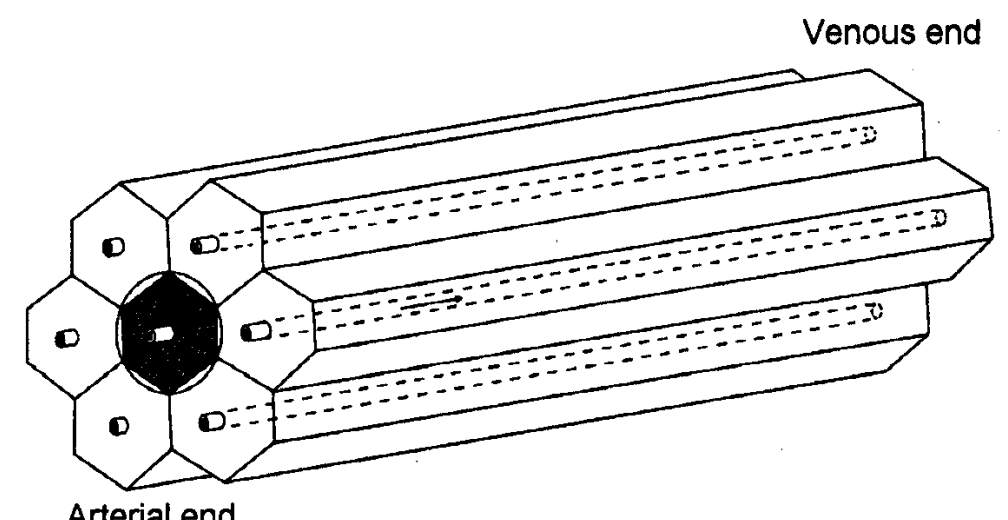

Arterial end

Figure 3.5: Theoretical configuration of capillaries in brain tissue; for optimal oxygen supply capillaries are arranged in a hexagonal lattice (adapted from $[18])$.

The oxygen consumption of brain matter is assumed to be independent of the local oxygen pressure. The cells may be represented as homogeneous volume distribution of sinks of oxygen, independent of time and position and the diffusion coefficient is assumed to be homogeneous.

\subsubsection{Theory of oxygen transport}

To calculate the oxygen supply of the tissue the mean oxygen pressure in the brain tissue is needed. The underlying process can be divided into two parts. In the first part, the oxygen pressure in blood has to be determined, whereas in the second part the diffusion of oxygen into and within the tissue is calculated.

These processes are derived in the following sections from simple assumptions and principles.

\section{Theory of oxygen transport: part 1}

As a starting point for the calculations of the oxygen pressure in blood, the equation of continuity is used:

$$
\frac{\partial \rho}{\partial t}+\operatorname{div} j=\alpha \quad \text { with } \quad j=\rho \cdot v
$$

\footnotetext{
${ }^{\dagger}$ As one is only interested in the mean oxygen pressure, it does not matter, because the integral over the axial and the radial diffusion is the same (compare [11]).
} 
where $\alpha$ describes the oxygen consumption of the surrounding tissue:

$$
\alpha=-\frac{\pi\left(r_{t}^{2}-r_{c}^{2}\right)}{\pi r_{c}^{2}} \cdot A
$$

It is assumed, that the tissue has a constant metabolism, which is given by $A\left[\frac{\mathrm{um}^{3} \mathrm{O}_{2}}{\mathrm{\mu m}^{3} \cdot \mathrm{s}}\right]$. For simplicity $\rho=\mathrm{CbO}_{2}$ is assumed. If the capillary is arranged in the $z$-direction, with $z=0$ denoting the beginning of the capillary, a stationary solution with $\frac{\partial \rho}{\partial t}=0$ and $\operatorname{div} j=\frac{d j}{d z}$ for the boundary condition $\mathrm{CaO}_{2}=\mathrm{CbO}_{2}(z=0)$ can be derived:

$$
\mathrm{CbO}_{2}(z)=\mathrm{CaO}_{2}+\alpha \cdot \frac{z}{v}
$$

To calculate the diffusion of oxygen into the tissue, the amount of dissolved oxygen and the partial pressure of oxygen in blood $p_{c}(z)$, respectively, is needed. This can be achieved by combining eq. 3.26 and eq. 3.27:

$$
\mathrm{CbO}_{2}(z)=\beta \cdot \frac{\left(\frac{p_{c}(z)}{p_{50}}\right)^{n}}{1+\left(\frac{p_{c}(z)}{p_{50}}\right)^{n}}+\gamma \cdot p_{c}(z)
$$

Rearranging eq. 3.31, so that it has the form of a polynomial equation, $p_{c}(z)$ can be provided, by determining the root of the following equation:

$$
\frac{\gamma}{p_{50}^{n}} \cdot p_{c}^{n+1}(z)-\frac{\mathrm{CbO}_{2}(z)-\beta}{p_{50}^{n}}+\gamma \cdot p_{c}(z)-\mathrm{CbO}_{2}(z)=0
$$

Here, $\mathrm{CbO}_{2}(z)$ can be expressed with eq. 3.30. Unfortunately, there is no analytical solution, whereof a solution for $\mathrm{CbO}_{2}(z)$ must be derived numerically. But an analytical solution can be derived, if the linear contribution of the dissolved oxygen in blood is ignored. As the dissolved oxygen amounts only $2 \%$ to the partial pressure, its contribution can be neglected as a first approximation. Then, by determining the saturation of haemoglobin in blood

$$
\mathrm{SbO}_{2}(z)=\frac{\left(\frac{p_{c}(z)}{p_{50}}\right)^{n}}{1+\left(\frac{p_{c}(z)}{p_{50}}\right)^{n}}
$$

and using the analog of eq. 3.30 for the haemoglobin saturation

$$
\mathrm{SbO}_{2}(z)=\mathrm{SaO}_{2}-\frac{r_{t}^{2}-r_{c}^{2}}{r_{c}^{2}} \frac{A}{v} \cdot z
$$

the partial pressure of oxygen can be determined:

$$
p_{c}(z)=p_{50} \cdot\left(\frac{\mathrm{SbO}_{2}(z)}{1-\mathrm{SbO}_{2}(z)}\right)^{\frac{1}{n}}
$$

Now the diffusion of oxygen into the tissue can be calculated by using the partial oxygen pressure in blood. 


\section{Theory of oxygen transport: part 2}

The oxygen diffusion into the tissue itself can be described by Fick's second law and using $j=-D \nabla p$ :

$$
\frac{\partial p}{\partial t}=D \cdot \Delta p-\frac{A}{c}
$$

whereas by parameter $D$ the $\mathrm{O}_{2}$ diffusivity is given, $A$ describes the $\mathrm{O}_{2}$ consumption and $c$ the $\mathrm{O}_{2}$ solubility. As axial diffusion can be ignored (compare section 3.2.2), eq. 3.36 can be rewritten using cylindrical coordinates. Assuming that the tissue is homogeneous and therefore gradient independent of $\varphi$, it follows:

$$
\frac{\partial p}{\partial t}=D \cdot\left(\frac{\partial p}{\partial r^{2}}+\frac{1}{r} \frac{\partial p}{\partial r}\right)-\frac{A}{c}
$$

This equation is then solved with the following boundary conditions:

$$
p=p_{c} \quad \text { at } \quad r=r_{c} \quad \text { and } \quad \frac{\partial p}{\partial r}=0 \quad \text { at } \quad r=r_{t}
$$

Therefore, the solution (for steady state) is:

$$
p_{t i}(r, z)=p_{c}(z)-\frac{A}{2 D c}\left(r_{t}^{2} \ln \frac{r}{r_{c}}+\frac{1}{2}\left(r_{c}^{2}-r^{2}\right)\right)
$$

To get the mean partial pressure in tissue $\bar{p}_{t i}$ one has to integrate over the tissue cylinder:

$$
\bar{p}_{t i}=\frac{1}{V_{t i}} \int_{0}^{L} \int_{0}^{2 \pi} \int_{r_{c}}^{r_{t}} p_{t i}(r, z) \cdot r d r d \phi d z
$$

whereas $V_{t i}$ is the volume of the tissue cylinder. Eq. 3.40 only holds, if $\bar{p}_{t i}$ is always positive, which for normal physiological conditions, as are assumed for the calculations, is always true. With eq. 3.39 and 3.40 it follows:

$$
\bar{p}_{t i}=\frac{1}{L} \int_{0}^{L} p_{c}(z) d z-\frac{A}{8 D c} \cdot \frac{-r_{t}^{4}+4 r_{c}^{2} r_{t}^{2}-3 r_{t}^{4}+4 r_{t}^{4} \ln \frac{r_{t}}{r_{c}}}{r_{t}^{2}-r_{c}^{2}}
$$

Using eq. 3.32 the integral over $p_{c}(z)$ can be solved numerically, but $p_{c}(z)$ has to be determined for every integration step. To avoid these extra computations an approximation can be derived using eq. 3.35 and 3.34, by ignoring the contribution of dissolved oxygen in blood:

$$
\bar{p}_{t i}=\frac{p_{50}}{L} \int_{0}^{L}\left(\frac{a-b z}{1-(a-b z)}\right)^{\frac{1}{n}} d z-\frac{A}{8 D c} \cdot \frac{-r_{t}^{4}+4 r_{c}^{2} r_{t}^{2}-3 r_{t}^{4}+4 r_{t}^{4} \ln \frac{r_{t}}{r_{c}}}{r_{t}^{2}-r_{c}^{2}}
$$

with

$$
a=\mathrm{SaO}_{2} \text { and } b=\frac{r_{t}^{2}-r_{c}^{2}}{r_{c}^{2}} \frac{A}{v}
$$

As there exists no closed solution for arbitrary n, eq. 3.42 has to be solved numerically. 


\subsection{Connecting Part 1 and Part 2}

To actually calculate the partial oxygen pressure in blood, the velocity of the blood itself is still needed (compare eq. 3.28). After solving the differential system in part 1 for a point in time $t$, the cerebral blood flow $(\mathrm{CBF})$ at this point in time can be calculated. The $\mathrm{CBF}$ is equivalent to:

$$
\mathrm{CBF}(t)=q_{A C}(t)=\frac{p_{A}(t)-p_{C}(t)}{R_{A C}(t)}
$$

The velocity of blood can then be determined from the CBF. This value for $v$ is used to calculate the oxygen diffusion into the tissue. Part 1 and part 2 will therefore be connected via the cerebral blood flow, which is sketched in figure 3.6.

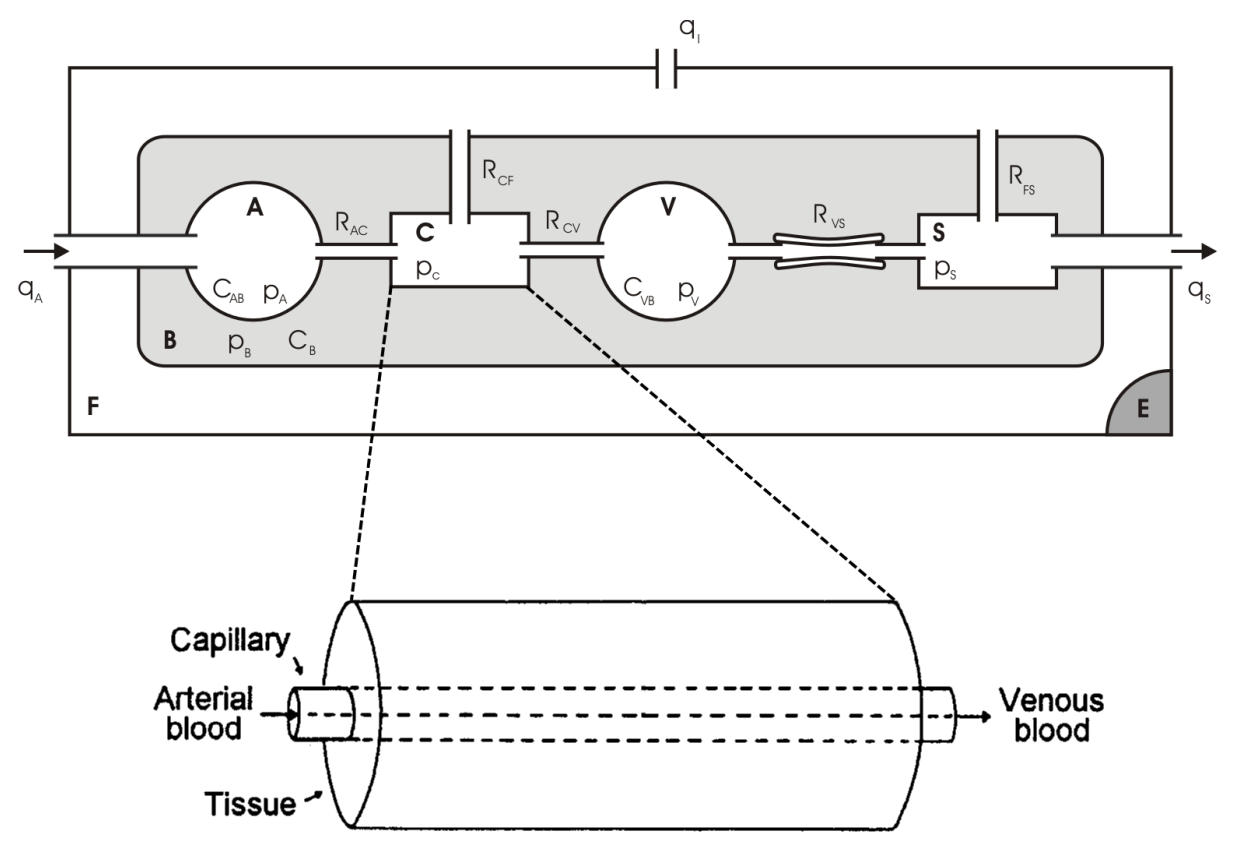

Figure 3.6: Connection between compartment model and oxygen transport.

As the (mean) partial oxygen pressure $\left(\mathrm{pbO}_{2}\right)$ in tissue itself can be measured with a Clark-type probe, the so called licox sensor, it is possible to verify the calculations of the model. It can be compared to real measured data, which will be done in chapter 8 . 


\subsection{Extensions and modifications}

As mentioned before the model has its limitations in reproducing measured data. Some of these limitations result from incomplete knowledge about the state of the brain, because of missing measurements, and the simplicity of the model. Therefore, extensions and modifications to the original model are necessary.

\subsubsection{Starling Resistor}

Due to high brain pressure, it is possible, that a situation occurs, in which the venous system can collapse. The original model is not able to simulate this state. Therefore, one has to alter the model to be able to simulate this important state of the brain. A common way is to assume, that the intracranial venous system behaves like a starling resistor [30].

Therefore, instead of being dependent on the volume $V_{V}$, the resistance $R_{V S}$ depends on the pressure differences between the veins and the sinus, and the veins and the brain pressure, respectively:

$$
R_{V S}= \begin{cases}\frac{1}{g_{V S}} \frac{p_{V}-p_{S}}{p_{V}-p_{B}} & \text { if }\left(p_{V}-p_{B}\right)>0 \\ \infty & \text { else }\end{cases}
$$

where $g_{V S}$ is the conductance between veins and sinus.

For a correct behavior of the model, the compliance of the veins has to be altered and can no longer be calculated with eq. 3.12. Instead, the compliance must be determined by the volume of the veins and the difference of the pressure inside the veins and the CSF pressure:

$$
C_{V B}=\frac{V_{V}}{p_{V}-p_{B}}
$$

To compare both scenarios, namely with and without the starling resistor, which will be done in chapter 6 , this value has to be adjusted in such a way, that $R_{V S}$ in the pressure dependent case and in the starling case are identical, if the system is in equilibrium.

\subsubsection{Adjusting the Model}

As the human brain consists of a left and right hemisphere, it is proposed to alter the model in such a way, that it reflects this structural condition.

\section{New Model}

Fig. 3.7 shows the new layout of the model. As before oxygen saturated blood reaches the brain through the main arteries. But then the inflow $q_{A}$ 


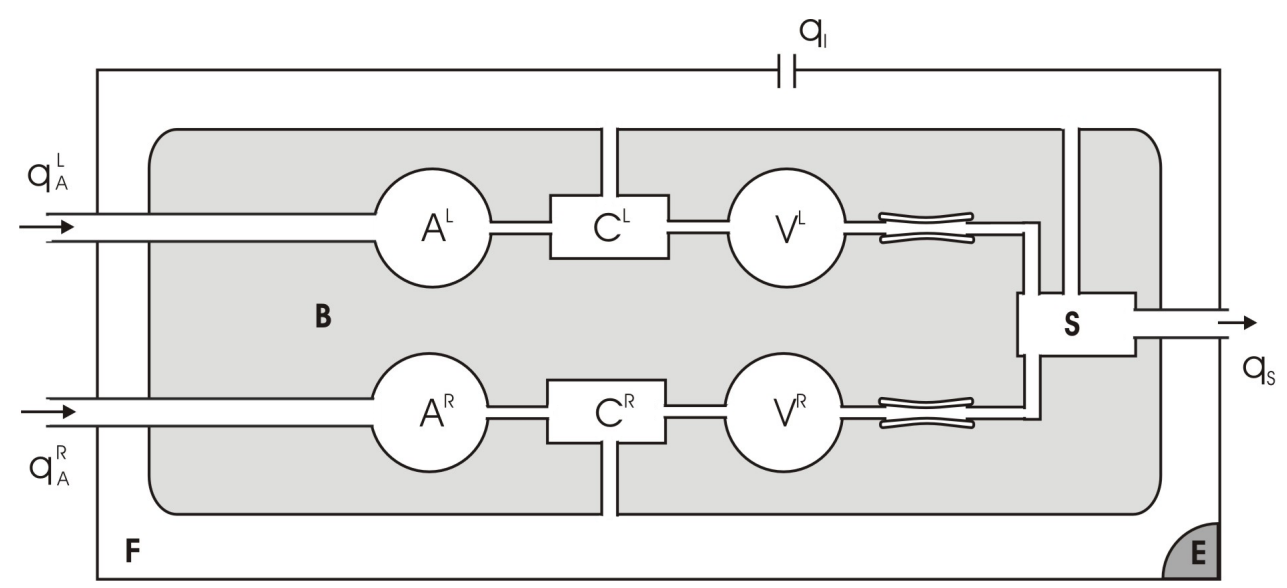

Figure 3.7: Extended Model; contrary to the old model (compare figure 3.1) the new model has 2 inflows (one for each hemisphere) and two separated vascular beds, which reunite at the compartment describing the sinuses. The superscript $L$ and $R$ denotes the left and the right hemisphere of a human brain. In addition, both hemispheres (see compartments $\mathrm{C}^{L}$ and $\mathrm{C}^{R}$ ) contribute to the CSF production.

splits up into two fluxes $q_{A}^{i}$ to supply the left or right hemisphere with blood. To calculate the new fluxes $q_{A}^{i}$ the following approach can be used.

Because fluxes in hydrodynamics behave like current in electrodynamics the whole resistance of the parallel connection is given by:

$$
R=\left(\frac{1}{R^{L}}+\frac{1}{R^{R}}\right)^{-1}=\frac{R^{L} \cdot R^{R}}{R^{L}+R^{R}}
$$

where $R^{i}=R_{A C}^{i}+R_{C V}^{i}+R_{V S}^{i}$ and $i \in\{L, R\}$. The superscript $L$ and $R$ denotes the left and the right hemisphere of a human brain. Since the sum of the inflows into the left and right hemisphere has to equal the inflow into the system, $q_{A}=q_{A}^{L}+q_{A}^{R}$ can be written as:

$$
q_{A}=\frac{p_{A}^{L} \cdot R^{R}+p_{A}^{R} \cdot R^{L}}{R^{L}+R^{R}}
$$

which determines the inflow to compartment $\mathrm{A}^{i}$.

An alternative approach can be derived by taking the anatomical conditions into account. As described in chapter 2, there exists a mechanism to guarantee a steady supply of oxygen to each area inside the brain: the circle of Willis. Considering the redundancies in the cerebral blood flow, it can be assumed, that the pressure in every artery, which branches from the circle, is identical. Therefore, $p_{A}^{L}=p_{A}^{R}$ can be used as input for the calculations in the model. The last approach is used for simulations in this work. 
As depicted in fig. 3.7 both hemispheres contribute to the CSF production. Compartment $\mathrm{S}$ now has two inflows from the venous system, which are the outflows of each hemisphere. The arterial system, capillaries and venous system of both hemispheres are surrounded by brain tissue, which itself is surrounded by the CSF, as before.

\section{New set of equations}

The new set of equations for the cerebral circulation can now easily be derived from the old ones. Because of the splitting into a left and right part there are three additional equations for the Compartments A, C and V. Extra flows and changes in mass have to be added were needed. The volume and the resistance of the veins has to be calculated separately for each compartment, but not the volume of the fluid compartment and its outflow resistance, as there is only one. The autoregulation mechanism of one hemisphere is modeled independently of the other one, the capacitances of the appropriate arteries have to be determined separately. Accordingly, the old set of equations transforms the following way:

$$
\begin{aligned}
A^{i}: & q_{A}^{i}-q_{A C}^{i}=\dot{C}_{A B}^{i} \Delta p_{A B}^{i}+C_{A B}^{i} \Delta \dot{p}_{A B}^{i} \\
C^{i}: & q_{A C}^{i}-q_{C B}^{i}-q_{C V}^{i}=0 \\
V^{i}: & q_{C V}^{i}-q_{V S}^{i}=C_{V B}^{i} \Delta \dot{p}_{V B}^{i} \\
M K: & q_{I}+q_{E}+\sum_{i} q_{C B}^{i}-q_{B S}= \\
& C_{B} \dot{p}_{B}-\sum_{i}\left(\dot{C}_{A B}^{i} \Delta p_{A B}^{i}+C_{A B}^{i} \Delta \dot{p}_{A B}^{i}+C_{V B}^{i} \Delta \dot{p}_{V B}^{i}\right)(3.5 \\
S: & \sum_{i} q_{V S}^{i}-q_{S}+q_{B S}=0
\end{aligned}
$$

with $R_{V S}^{i}=R_{V S}^{i}\left(V_{V}^{i}\right), R_{F S}=R_{F S}\left(p_{B}-p_{S}, V_{F}\right)$ and

$$
\begin{gathered}
\frac{d}{d t} V_{V}^{i}=q_{C V}^{i}-q_{V S}^{i}, \frac{d}{d t} V_{F}=q_{I}+\sum_{i} q_{C B}^{i}-q_{B S} \\
\frac{d C_{A B}^{i}}{d t}=-\frac{1}{\tau}\left(C_{A B}^{i}-C_{A B r e g}\left(\alpha^{i} \cdot x^{i}\right)\right) \text { with } x^{i}=\frac{q_{A C}^{i}-q_{A C n}}{q_{A C n}}
\end{gathered}
$$

Then, the partial CBF in each hemisphere can be determined and used for the calculations of oxygen transport to the tissue. 


\section{Chapter 4}

\section{Default values of the mathematical model}

To check the validity of the model, the parameters of the model must be chosen in a meaningful way. The simulations are supposed to produce results, which are comparable to known physiological behavior of the human brain in a qualitative manner. It is desired to not only understand the underlying processes, but also to be able to compare the results of the simulations itself to measured data sets. Therefore, the parameters have to be chosen in such a way, that the simulations produce results, which are directly comparable to real measured data in a quantitative way. This can be achieved by extracting known standard values from medical literature.

Unfortunately, there is no complete set of standard values, which can easily be used for the model. Due to natural variety, extracted values can only be specified for a range and even then, values from different sources differ. This means the parameters have to be determined in such a way, that the model is consistent in itself. In order to achieve this, a minimum set of parameters with meaningful values is extracted from the literature, so that the rest of the undetermined values can be calculated with the new determined parameters and this minimum set.

Because some values differ for the basic Haemodynamics and Oxygen transport Model (bHOM) and altered (sHOM)/extended (esHOM) models, at first the parameters are determined for the basic model. Using these values, the parameters for the extended model can be deduced in such a way, that both will produce equal results for the case, that the system is in equilibrium.

The parameters of the model, which represent the boundary conditions of the nonlinear system of equations, are a special case. In equilibrium, they are equal to the corresponding standard values, but they have to be determined separately in any other case, which has to be taken care of. Otherwise, the simulations may yield non-realistic results. 
As the autoregulation mechanism is a crucial part of the model, its parameters are discussed in a later section and their influence on the behavior of the model will be examined in more detail.

\subsection{Fixed parameters}

The parameters for the mentioned minimum set can be separated into five different types: volume, flux, constants, pressure and capacitance. In the following the chosen values for the fixed parameters of the model will be given.

\subsubsection{Volume}

Standard values for the volumes of each compartment can be extracted from medical literature. The here presented values are the same as in [11]:

$$
\begin{aligned}
& V_{B}=1000 \mathrm{ml}, \quad V_{A}=15 \mathrm{ml}, \quad V_{C}=10 \mathrm{ml}, \\
& V_{V}=40 \mathrm{ml}, \quad V_{S}=80 \mathrm{ml}, \quad V_{F}=30 \mathrm{ml}
\end{aligned}
$$

For the meaning of the subscripts, see figure 3.1.

For the simulation itself only the values for $V_{V}$ and $V_{F}$ are of interest. The other values are shown for comparison to see, how the volume differs for each compartment.

\subsubsection{Pressures}

For the pressure in the blood vessels and the pressure inside the brain and the cerebrospinal fluid (CSF), the following values are used (compare [7]):

$$
\begin{aligned}
& p_{A}=100 \mathrm{mmHg}, \quad p_{V}=15 \mathrm{mmHg}, \quad p_{B}=p_{F}=10 \mathrm{mmHg}, \\
& p_{C}=25 \mathrm{mmHg}, \quad p_{S}=5 \mathrm{mmHg}
\end{aligned}
$$

Here, the same units are used as in medical literature, which will allow a better comparison between values obtained in the simulations and results in other medical works. Figure 4.1 shows the distribution of the blood pressure, as it flows through the vascular bed. As mentioned before, brain and CSF pressure can be assumed to be equal.

\subsubsection{Fluxes}

For the flux $q_{C F}$, which describes the CSF production, the same value as in [11] is used. In an equilibrium state the same amount of CSF is absorbed as is produced and therefore the absolute values of the flux $q_{C F}$ and $q_{F S}$ are the same, but differ in sign:

$$
q_{C F}=0.4 \frac{\mathrm{ml}}{\mathrm{min}}, \quad q_{F S}=-0.4 \frac{\mathrm{ml}}{\mathrm{min}}
$$




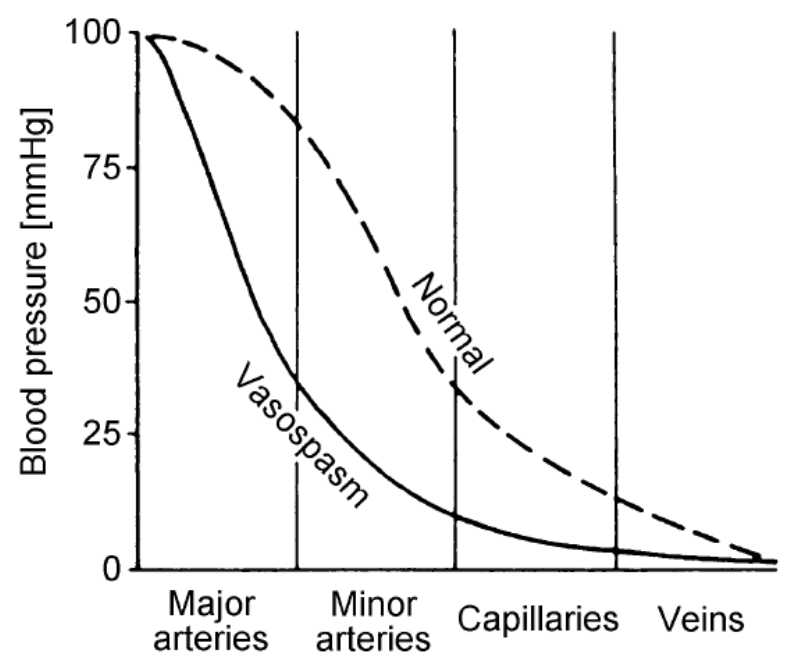

Figure 4.1: Blood pressure distribution of the vascular bed (adapted from [12]) for normal conditions and constricted blood vessels (vasospasm).

An established value for the cerebral blood flow (CBF) in literature, which in the model is identical to the flux from arteries to capillaries, is used as standard value:

$$
q_{A C}=\mathrm{CBF}=600 \frac{\mathrm{ml}}{\mathrm{min}}
$$

\subsubsection{Constants}

To calculate the capacitances for $C_{V B}$ (eq. 3.12) and $C_{B}$ (eq. 3.18) the following values for the constants are used, which are proposed by Ursino and Lodi in [29]:

$$
k_{V}=0.3 \frac{1}{\mathrm{ml}}, \quad k_{B}=0.26 \frac{1}{\mathrm{ml}}
$$

The values for the offsets in brain pressure and venous pressure are the same as used in [11]:

$$
p_{V_{0}}=2.5 \mathrm{mmHg}, \quad p_{B_{0}}=2.5 \mathrm{mmHg}
$$

\subsubsection{Capacitance}

The last parameter, which is needed for the minimal set, is the capacitance of the arteries $C_{A B}$. The standard value is:

$$
C_{A B n}=0.15 \frac{\mathrm{ml}}{\mathrm{mmHg}}
$$


which is given by Ursino and Lodi in [29].

In the next section the rest of the undetermined parameters will be deduced from this minimal set.

\subsection{Calculated parameters}

The complete set of parameters has to be chosen in such a way, that when the system starts in an equilibrium state the system remains in equilibrium, as long as there are no internal or external influences. This can be achieved by determining the remaining parameters in such a way, that all deviations in the nonlinear differential equation system are zero.

\subsubsection{Brain compartment}

With the default values for $k_{B}, p_{B}$ and $p_{B_{0}}$ and eq. 3.18 , the compliance of the brain tissue can be calculated:

$$
C_{B}=\frac{20}{39} \frac{\mathrm{ml}}{\mathrm{mmHg}} \approx 0.5128 \frac{\mathrm{ml}}{\mathrm{mmHg}}
$$

\subsubsection{CSF compartment}

Using the values for the fluxes $q_{C F}$ and $q_{F S}$ and pressures $p_{B}, p_{C}$ and $p_{S}$ of the previous section, the resistances $R_{C F}$ and $R_{F S}$ can easily be determined with eq. 3.2:

$$
\begin{aligned}
R_{C F} & =\frac{p_{C}-p_{B}}{q_{C F}} \\
R_{F S} & =\frac{p_{B}-p_{S}}{q_{F S}}
\end{aligned}
$$

The standard values for the corresponding resistances are then:

$$
R_{C F n}=37.5 \frac{\mathrm{mmHg}}{\mathrm{ml} / \mathrm{min}}, \quad R_{F S n}=12.5 \frac{\mathrm{mmHg}}{\mathrm{ml} / \mathrm{min}}
$$

\subsubsection{Artery compartment}

For the artery compartment, the parameters $k_{R_{A C}}$ and $R_{A C}$ have to be determined. With eq. 3.2, the standard values for $\mathrm{CBF}$, and pressures $p_{A}$ and $p_{C}$, the resistance

$$
R_{A C}=\frac{p_{A}-p_{B}}{\mathrm{CBF}}
$$

yields

$$
R_{A C n}=0.125 \frac{\mathrm{mmHg}}{\mathrm{ml} / \mathrm{min}}
$$


leading to the determination of the constant $k_{R_{A C}}$ by use of eq. 3.6:

$$
\begin{aligned}
k_{R_{A C}} & =R_{A C} C_{A B}^{2}\left(p_{A}-p_{B}\right)^{2} \\
& =\frac{729}{32} \frac{\mathrm{ml}^{2} \cdot \mathrm{mmHg}}{\mathrm{ml} / \mathrm{min}} \approx 22.781 \frac{\mathrm{ml}^{2} \cdot \mathrm{mmHg}}{\mathrm{ml} / \mathrm{min}}
\end{aligned}
$$

\subsubsection{Capillary compartment}

Using equation 3.20, which describes the in- and outflows of the capillary compartment, the resistance $R_{C V}$ can be calculated. It follows:

$$
R_{C V}=\frac{25}{1499} \frac{\mathrm{mmHg}}{\mathrm{ml} / \mathrm{min}} \approx 0.01668 \frac{\mathrm{mmHg}}{\mathrm{ml} / \mathrm{min}}
$$

\subsubsection{Vein compartment}

The last undetermined parameters are now: $k_{R_{V S}}, R_{V S n}, C_{V B n}$ and $g_{V S}$. In equilibrium the sum over the fluxes $q_{C V}$ and $q_{V S}$ has to be zero, as there can be no change in volume of the capillary compartment. Therefore, the standard value for the resistance $R_{V S n}$ yields:

$$
R_{V S n}=\frac{25}{1499} \frac{\mathrm{mmHg}}{\mathrm{ml} / \mathrm{min}} \approx 0.01668 \frac{\mathrm{mmHg}}{\mathrm{ml} / \mathrm{min}}
$$

Thus, the constant $k_{R_{V S}}$ has to be:

$$
k_{R_{V S}}=\frac{40000}{1499} \frac{\mathrm{ml}^{2} \cdot \mathrm{mmHg}}{\mathrm{ml} / \mathrm{min}} \approx 26.684 \frac{\mathrm{ml}^{2} \cdot \mathrm{mmHg}}{\mathrm{ml} / \mathrm{min}}
$$

To obtain the standard value for the capacitance $C_{V B}$ of the veins, eq. 3.12 is used. Its value is then:

$$
C_{V B n}=\frac{1}{k_{V}\left(\left|p_{V}-p_{B}\right|-p_{V_{0}}\right)}=\frac{20}{45} \frac{\mathrm{ml}}{\mathrm{mmHg}} \approx 0.4444 \frac{\mathrm{ml}}{\mathrm{mmHg}}
$$

In the starling case with

$$
R_{V S}=g_{V S} \frac{p_{V}-p_{S}}{p_{V}-p_{B}}
$$

it directly follows, that:

$$
g_{V S}=\frac{25}{1499} \frac{\mathrm{ml} / \mathrm{min}}{\mathrm{mmHg}} \approx 0.01668 \frac{\mathrm{ml} / \mathrm{min}}{\mathrm{mmHg}}
$$




\subsection{Boundary conditions for bHOM model}

The boundary conditions, which are used for the simulations as a starting point for equilibrium and are based on the values derived in the previous sections, are therefore:

\begin{tabular}{c|r|c} 
Parameter & Standard Value & Units \\
\hline$V_{E}$ & 0 & $\mathrm{ml}$ \\
$V_{F}$ & 30 & $\mathrm{ml}$ \\
$V_{V}$ & 40 & $\mathrm{ml}$ \\
$p_{B}$ & 10 & $\mathrm{mmHg}$ \\
$p_{V}$ & 15 & $\mathrm{mmHg}$ \\
$C_{A B}$ & 0.15 & $\frac{\mathrm{ml}}{\mathrm{mmHg}}$
\end{tabular}

To emphasize it again: If the starting point for the simulations is not equal to the equilibrium state, these values have to be adjusted.

\subsection{Adjustments for two hemispheres}

To compare the esHOM model with the bHOM or sHOM model, some of the parameters have to be adjusted, in order to make both models comparable. In the following the changes will be stated and explained. In section 4.4.2 the values for the boundary conditions are summarized.

\subsubsection{Adjusted values}

The changes to the standard values, which are needed for the two hemisphere model, can easily be summarized:

Fluxes: Like in a parallel circuit in electrodynamics, all fluxes into and from each hemisphere are halved. In particular, each hemisphere contributes only half the amount to the original CSF flux.

Resistance: As the pressure in each compartment stays the same, it follows, that the corresponding value of the resistance has to be doubled for each flux, that is halved.

Volume: The change in volume for all blood vessels in each hemisphere can be deduced from eq. 3.5. Therefore, it has to be divided by a factor $\sqrt{2}$.

Compliance: As the compliance is directly proportional to the volume, it follows from eq. 3.3 , that the compliance itself must also be divided by a factor of $\sqrt{2}$. 
For the simulation itself, a summary of the necessary altered standard values follows, whereas the subscript $n$ denotes, that a value is the standard value and the superscript $i$ is a placeholder for the left/right hemisphere:

$$
\begin{gathered}
R_{V S_{n}}^{i}=2 \cdot R_{V S_{n}} \\
R_{C V_{n}}^{i}=2 \cdot R_{C V_{n}}, \quad R_{C F_{n}}^{i}=2 \cdot R_{C F_{n}} \\
V_{V_{n}}^{i}=V_{V_{n}} / \sqrt{2} \\
C_{A B_{n}}^{i}=C_{A B_{n}} / \sqrt{2}
\end{gathered}
$$

One consequence of the modification to the volume of the venous compartment is, that the total volumes of the arterial and venous systems are approximately $42 \%$ bigger. This may seem to be in conflict with their standard volumes, given in section 4.1.1, but this modification can be justified. As already mentioned in the beginning of this chapter, standard values in literature vary. In [3] it is stated, that the ratio between the volume of the veins and arteries is about $2: 1$ and the total volume of both compartments is approximately $75 \mathrm{ml}$. There, the volume of the arteries is about $25 \mathrm{ml}$ and for the veins it is about $50 \mathrm{ml}$. According to these values, the new standard values for the volumes of the extended model, with $V_{A} \approx 21.2 \mathrm{ml}$ and $V_{V} \approx 56.6 \mathrm{ml}$, fit these assumptions better than the previously given standard values. Furthermore, the set of parameters has to be consistent, which led to this adjustment.

\subsubsection{Boundary conditions (two hemispheres)}

The boundary conditions for two hemispheres are therefore the same as in the basic model, except for the values for the volume of the veins $V_{V}$ and the compliance of the arteries $C_{A B}$. For these the values of the basic model have to be divided by the square root of two:

\begin{tabular}{c|r|c} 
Parameter & Two hemispheres & Units \\
\hline$V_{E}$ & 0 & $\mathrm{ml}$ \\
$V_{F}$ & 30 & $\mathrm{ml}$ \\
$V_{V}^{i}$ & $40 / \sqrt{2}$ & $\mathrm{ml}$ \\
$p_{B}$ & 10 & $\mathrm{mmHg}$ \\
$p_{V}^{i}$ & 15 & $\mathrm{mmHg}$ \\
$C_{A B}^{i}$ & $0.15 / \sqrt{2}$ & $\mathrm{ml}$ \\
\hline $\mathrm{mHg}$
\end{tabular}

It should be mentioned, that the number of boundary conditions is raised from 6 to 9 , due to the extra hemisphere. 


\subsection{Parameters for autoregulation}

As the autoregulation describes an aspect of the human brain, its values are not part of the minimal set. The minimal set was chosen in such a way, that the system remains in equilibrium, if there are no external or internal changes and therefore, no autoregulation is needed. But for the sake of completeness the used values are given.

The standard values for the parameters of the autoregulation used in the simulations in case of the bHOM model are:

$$
\begin{array}{cl}
\alpha=2, & \tau=10 \mathrm{~s} \\
\Delta C_{A B 1}=0.165, & \Delta C_{A B 2}=0.065
\end{array}
$$

For the esHOM model with two hemispheres not only $\Delta C_{A B x}$ has to be altered, in order to have the same range of the autoregulation, but $\alpha$ and $\tau$ have to be adjusted as well (compare chapter 7 ). The used values are:

$$
\begin{aligned}
\alpha^{i}=1.4, & \tau^{i}=2.5 \mathrm{~s} \\
\Delta C_{A B 1}^{i}=0.119, & \Delta C_{A B 2}^{i}=0.046
\end{aligned}
$$

for each hemisphere $i$. It should be mentioned, that in the extended model it is possible to use different parameters for each hemisphere, which should fit the natural fluctuations in the cerebral blood vessels system better. But for the sake of simplicity this is only done in the case of $\alpha$, to simulate a situation, in which the autoregulation is impaired in one hemisphere while still working in the other (see chapter 7).

\subsubsection{Behavior of Autoregulation}

Here, the influence of the free parameters in the autoregulation in the simulations are examined. There are four tunable parameters, namely:

- $\alpha$, which describes the state of the autoregulation: active $(\alpha>0)$ or impaired autoregulation $(\alpha=0)$.

- $\tau$, a time constant of the autoregulation mechanism.

- $\Delta C_{A B}$, which determines the range of the autoregulation curve and

- $q_{A C n}$, which determines the point of the CBF, in which the system is in equilibrium.

The right values for the parameters are crucial to simulate the right behavior of the brain. Unfortunately, it is not possible to extract meaningful values for those parameters from the literature, as the autoregulation mechanism in the model is a mathematical abstraction of the natural mechanism. Its values had to be determined by comparison with known behavior of the brain in experiments and common knowledge. As their general influence is also of high interest, in the following they are examined in more detail in the case of the basic model. 


\section{Influence of $\Delta C_{A B x}$}

To see the influence of $\Delta C_{A B}$ in figure 4.2, the arterial blood pressure (ABP) is plotted against the $\mathrm{CBF}$ for an active autoregulation $(\alpha=2)$ and different $\triangle C_{A B 1}$ and $\triangle C_{A B 2}$ :

\begin{tabular}{c|r|r} 
Run & $\Delta C_{A B 1}$ & $\Delta C_{A B 2}$ \\
\hline 1 & 0.200 & 0.040 \\
2 & 0.165 & 0.065 \\
3 & 0.130 & 0.090
\end{tabular}

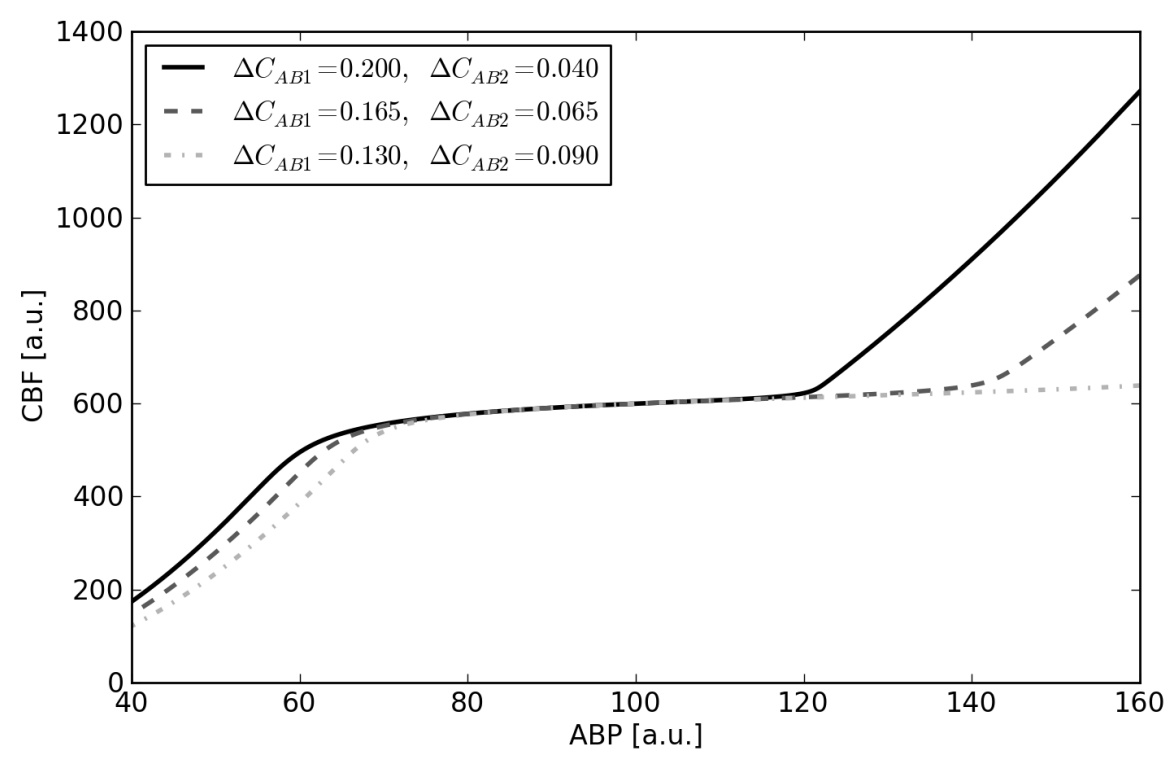

Figure 4.2: $\mathrm{CBF}$ against $\mathrm{ABP}$ for $\alpha=2$ and different $\Delta C_{A B x}$, done with the bHOM model; the influence of each $\Delta C_{A B x}$ is independent of each other.

As the influence of $\Delta C_{A B 1}$ is independent of $\Delta C_{A B 2}$ and vice versa, which can easily be verified by eq. 3.8, different values are used in each graph for a compact view. As it can be seen, a bigger value of $\Delta C_{A B x}$ results in an extension of the plateau, where the CBF is nearly constant. Therefore, the values can easily be adjusted as needed, to model the behavior extracted from experiments or common knowledge.

The behavior of the autoregulation does neither differ for the starling case nor for two hemispheres and is therefore not shown for convenience. The corresponding values for $\Delta C_{A B}$ can be calculated with the equations, in order to get comparable simulations for $\mathrm{bHOM} / \mathrm{sHOM}$ and esHOM models. 


\section{Influence of $\alpha$}

As mentioned before, the purpose of the autoregulation is to achieve a constant blood flow. In this section the influence of $\alpha$ is examined. This is done for the bHOM model. As there is no fundamental difference in the functioning of the autoregulation mechanism for the sHOM or the esHOM model (two hemispheres), those scenarios are neglected for convenience.

Fig. 4.3 shows the autoregulation mechanism for different values of $\alpha$ for the bHOM model. Here, $\Delta C_{A B 1}=0.165$ and $\Delta C_{A B 2}=0.065$ are used.

For an $\mathrm{ABP}$ of $100 \mathrm{mmHg}$ and a $\mathrm{CBF}$ of $600 \mathrm{ml} / \mathrm{min}$ all graphs intersect. At this point the term caused by the autoregulation vanishes, because the $\mathrm{CBF}$ equals the reference point for the autoregulation. As this marks the ideal value, no regulation has to be done. Also the effect of $\alpha$ is obvious. With increasing $\alpha$ a plateau is developed that gets flatter and broader, until the maximum width is reached. In this case the capability of the autoregulation to keep blood flow constant is limited to the region between an $\mathrm{ABP}$ of approximately 60 and $140 \mathrm{mmHg}$.

Fig. 4.4 shows the corresponding resistances $R_{A C}$. Here, the influence of $\alpha$ on the capacitance and therefore on the resistance can easily be seen. In respect to the standard value, the resistance increase/decrease raises with increasing $\alpha$, which is responsible for the flattening of the plateau mentioned earlier. In an analog way to the CBF for different $\alpha$ all graphs intersect for an $\mathrm{ABP}$ of $100 \mathrm{mmHg}$, as there the autoregulation term of the compliance $C_{A B}$ vanishes.

\section{Influence of $\tau$}

Until now, the influence of the deviation of the ABP has been neglected. If the ABP changes too quickly, the regulation circuit is too slow to compensate this change. This lag can be tuned by the parameter $\tau$. Figures 4.5 and 4.6 show the CBF against ABP for different $\alpha$, in which $\tau$ has been chosen in such a way, that the autoregulation mechanism can lag behind. This results in a hysteresis like behavior of the $\mathrm{CBF}$ graphs.

As the magnitude of $\tau$ is reduced from the largest value to the smallest, the lag is reduced up to a point, for which with smaller values of $\tau$ no further improvement is achieved $(\tau \approx 10 \mathrm{~s})$. This can easily be explained: for a certain value of $\tau$ the regulation mechanism has enough time to react on a change in $\mathrm{ABP}$ and compensate this change within the range of the autoregulation. If one reduces this value of $\tau$, there can be no improvement, as the optimal regulation has already been achieved within the time limit and therefore, there is no noticeable difference in the CBF. 


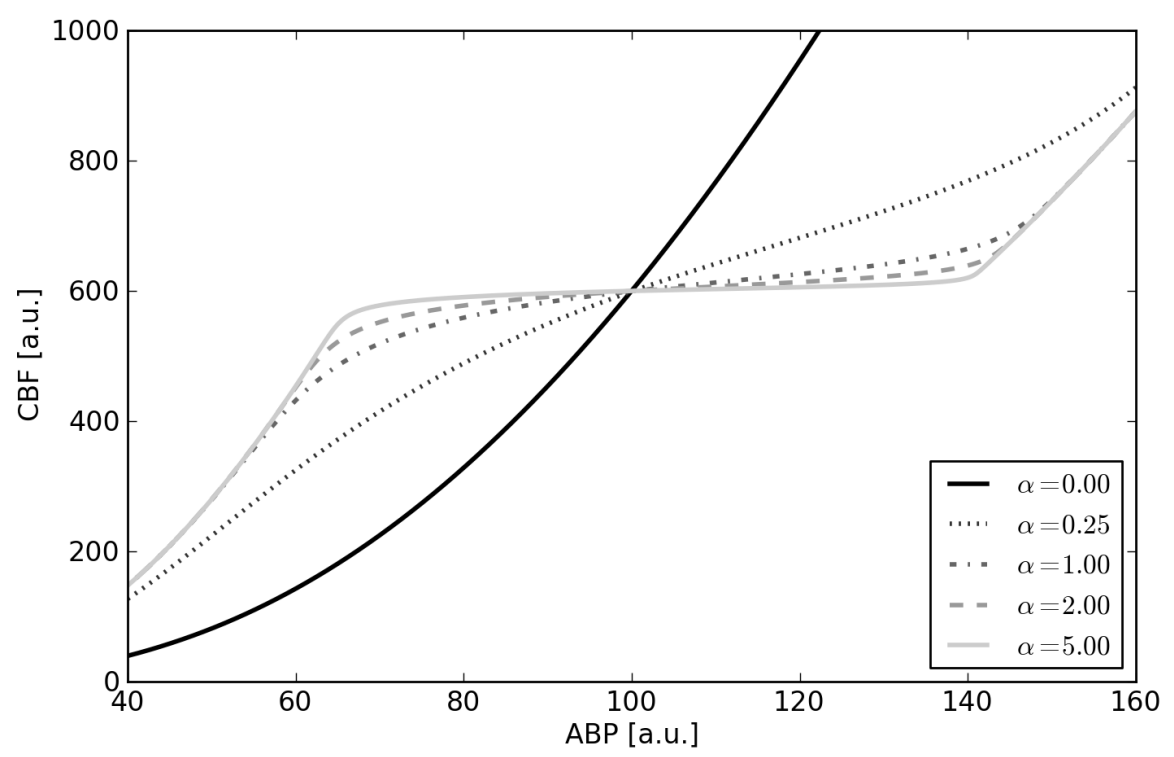

Figure 4.3: $\mathrm{CBF}$ against $\mathrm{ABP}$ for different $\alpha$ (bHOM).

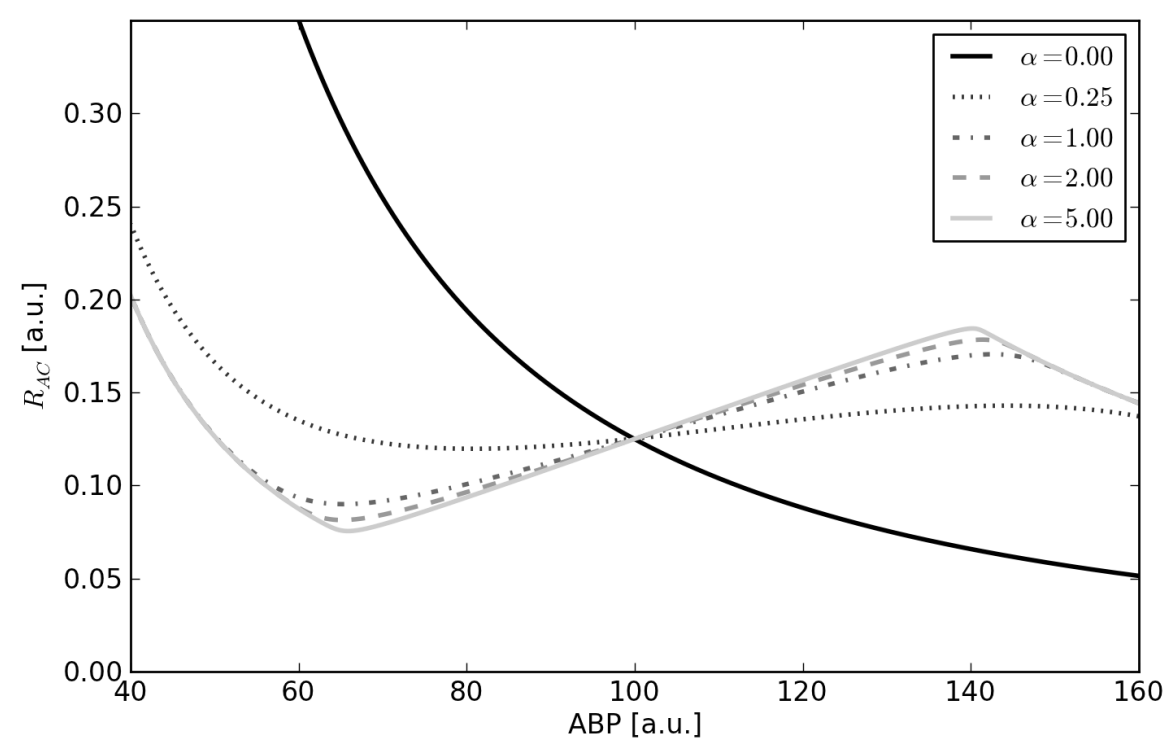

Figure 4.4: $R_{A C}$ against ABP for different $\alpha$ (bHOM). 


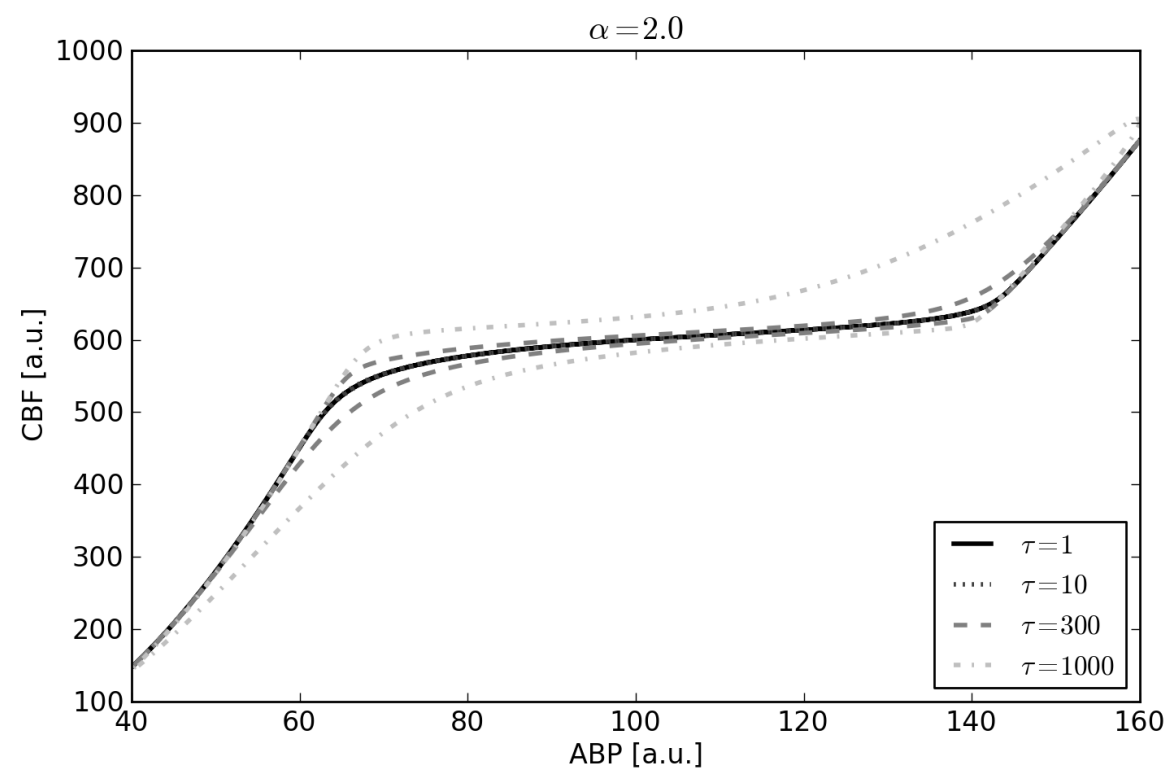

Figure 4.5: CBF against $\mathrm{ABP}$ for fixed $\alpha=2.0$ and different $\tau[\mathrm{s}](\mathrm{bHOM})$.

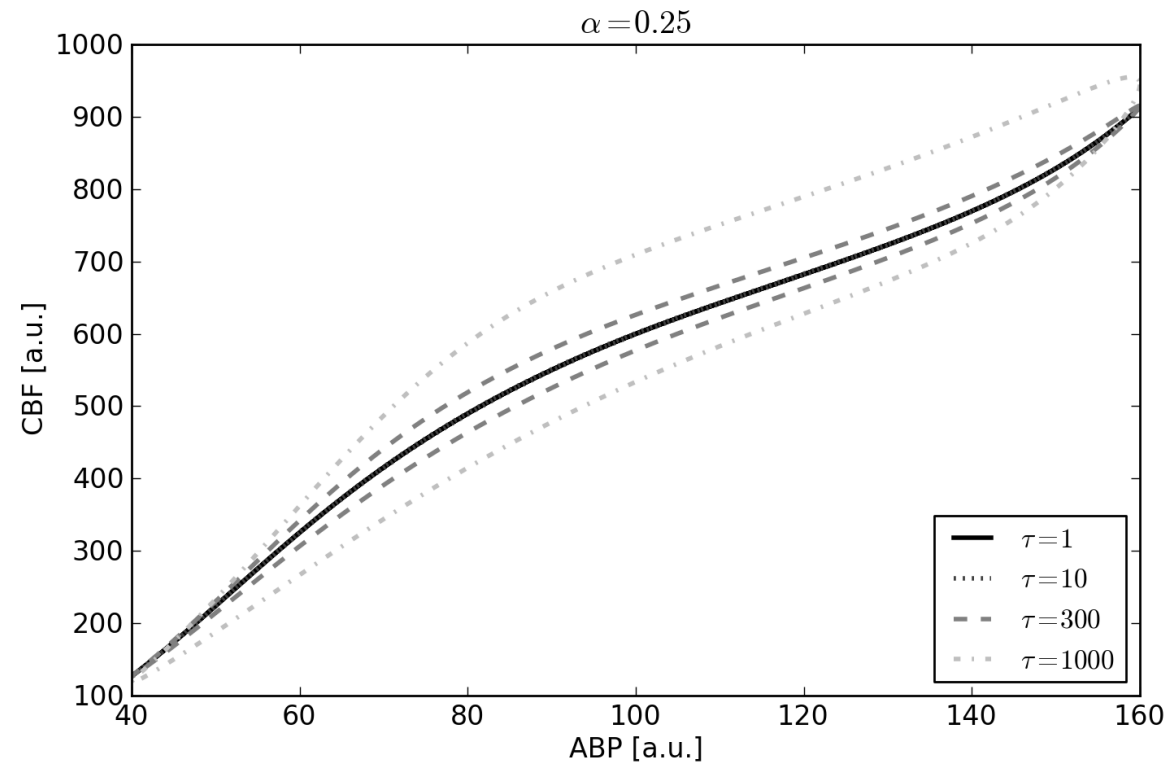

Figure 4.6: CBF against ABP for fixed $\alpha=0.25$ and different $\tau[\mathrm{s}](\mathrm{bHOM})$. 


\section{Point of autoregulation}

As already mentioned in the previous section, the default value for the $\mathrm{CBF}$ is about $600 \mathrm{ml} / \mathrm{min}$. If the $\mathrm{CBF}$ is different from this value and the autoregulation is not impaired, the blood flow through the system will be regulated in such a way, that the $\mathrm{CBF}$ will be brought as close as possible to the standard $\mathrm{CBF}$ value, with respect to the constrains. As the range of the autoregulation is limited, a CBF outside of this range will be altered towards the standard $\mathrm{CBF}$ by the autoregulation as much as it is possible. The range of the regulation mechanism is described by $\Delta C_{A B}$.

\subsection{Oxygen transport}

The determination of the minimal set of parameters for the oxygen transport is easier, as there are no parameters, which have to be calculated.

As in chapter 3 , the set of parameters can be split into different parts, namely the parameters to determine the

- partial oxygen pressure in blood,

- properties of the Krogh cylinder,

- partial oxygen pressure in the tissue,

as will be done in the following sections.

\subsubsection{Partial oxygen pressure in blood}

To solve the equation for the partial oxygen pressure in blood the values for $n$ and $p_{50}$ are needed. In the literature (compare [20], [23] and [33]) values for $n$ vary between 2.5 and 2.7 and for $p_{50}$ between 26 and $27.6 \mathrm{mmHg}$. Here, the following values are used (as in [11]):

$$
n=2.6 \quad \text { and } \quad p_{50}=26 \mathrm{mmHg}
$$

For the sake of completeness the values needed for calculating the total amount of oxygen used in eq. 3.26 are repeated:

$$
\begin{array}{r}
\beta=0.201 \frac{\mathrm{cm}^{3} \mathrm{O}_{2}}{\mathrm{~cm}^{3}} \\
\gamma=3 \cdot 10^{-5} \frac{\mathrm{cm}^{3} \mathrm{O}_{2}}{\mathrm{~cm}^{3} \mathrm{mmHg}}
\end{array}
$$

which are given in [22]. 


\subsubsection{Properties of Krogh cylinder}

To determine the oxygen transport from the capillaries into the tissue, the parameters of the Krogh cylinder are needed, such as size and density of the capillaries and the surrounding tissue.

Due to [2] the radius for capillaries is around $3.5 \mu \mathrm{m},{ }^{*}$ but their length is hard to determine. A range of $600-1050 \mu \mathrm{m}$ is assumed in [25]. In this work, the same value as in [11] is used, which is $L=770 \mu \mathrm{m}$.

The density of capillaries in grey matter is around 1000-1100 capillaries per $\mathrm{mm}^{2}$, whereas 300 capillaries $/ \mathrm{mm}^{2}$ is typical for white matter (see [16]). As the sensor ${ }^{\dagger}$ for measuring the partial oxygen pressure in tissue is placed into white matter, the appropriate capillary density is used. Here, a value of 330 capillaries $/ \mathrm{mm}^{2}$ is assumed as in [11].

The values for the Krogh cylinder are therefore:

$$
r_{c}=3.5 \mu \mathrm{m}, \quad r_{t}=31 \mu \mathrm{m} \hat{=} 330 \frac{\text { capillaries }}{\mathrm{mm}^{2}} \text { and } \quad L=770 \mu \mathrm{m}
$$

The last value needed for calculating the oxygen transport from the capillaries to the tissue is the velocity of the blood. Under normal conditions the value of the velocity of blood is around

$$
v=400 \frac{\mu \mathrm{m}}{\mathrm{s}}
$$

as stated in [14].

\subsubsection{Partial oxygen pressure in tissue}

For the calculations of the partial oxygen pressure in the tissue the values for oxygen consumption, diffusivity and solubility are needed. Their values can be extracted from [1] and [14]:

$$
\begin{array}{r}
A=4.5 \cdot 10^{-4} \frac{{\mu \mathrm{m}^{3} \mathrm{O}_{2}}^{\mu \mathrm{m}^{3} \cdot \mathrm{s}}}{D=1.8 \cdot 10^{3} \frac{\mu \mathrm{m}^{2}}{\mathrm{~s}}} \\
c=2.6 \cdot 10^{-5} \frac{\mu \mathrm{m}^{3} \mathrm{O}_{2}}{\mu \mathrm{m}^{3} \cdot \mathrm{mmHg}}
\end{array}
$$

\subsection{Summary}

In this chapter a complete set of meaningful parameters is given, which makes it possible to simulate the haemodynamical processes and oxygen transport inside the human brain and compare the results with real world measurements, which will be done in the next chapters.

\footnotetext{
${ }^{*}$ Remark: As the size of blood cells is around $7.7 \mu \mathrm{m}$, the blood cells are squeezed, when going through capillaries.

${ }^{\dagger}$ The so called licox sensor.
} 


\section{Chapter 5}

\section{General behavior of the bHOM model}

As the general validity of the model has already been confirmed in [11], it is of general interest, to see how the model behaves for different parameter settings. With this knowledge, one should be able to make predictions for the behavior of the brain under specific physiological conditions.

Therefore, in this chapter the behavior of the basic Haemodynamics and Oxygen transport Model (bHOM) is examined in more detail, in order to understand, what the underlying mechanisms are, that are responsible for the behavior of the system.

To examine the behavior of the model, in the next sections a closer look will be taken, how each compartment in the simulated system behaves in general. Thus, different simulations are prepared, in which at least one aspect of the system is altered.

At first, the simulations were done without the autoregulation mechanism enabled $(\alpha=0)$, as for this scenario the analysis of the system will be the easiest from a model point of view. Then, the complexity of the model is increased by analyzing the system with an enabled autoregulation $(\alpha=2)$, as this scenario represents the standard conditions of the human brain. The same analyses are made with and without the autoregulation for an occurrence of a swelling, to simulate the state of a patient with a severe head injury.

\subsection{Overview}

For simplicity, all simulations are done with a sinusoidal arterial blood pressure (ABP) with an amplitude of $60 \mathrm{mmHg}$, a period of 15000 and for 30000 points in time. The starting point of the ABP lies at $100 \mathrm{mmHg}$, which will also be the starting point of the discussion, if not stated otherwise. For the analyses of the simulations, the development of the system can be divided 
into four different time segments. Each segment consists of 3750 points in time and the whole pattern is repeated every 15000 points in time, due to the periodicity of the used ABP.

The segments are divided into the following intervals, in which the ABP is between:

1. 100 and $160 \mathrm{mmHg}$, raising

2. 100 and $160 \mathrm{mmHg}$, falling

3. 40 and $100 \mathrm{mmHg}$, falling

4. 40 and $100 \mathrm{mmHg}$, raising

To be able to compare all simulations with different aspects/parameters, this setup will be used for all simulations with an artificial ABP as input throughout this work.

The amplitude of $60 \mathrm{mmHg}$ for the $\mathrm{ABP}$ was chosen is such a way, that the ABP reaches levels outside the scope of an intact autoregulation mechanism. This can happen under natural conditions of the human physiology and will also allow a better understanding of the autoregulation.

\section{$5.2 \quad$ No Swelling}

In this section the behavior of the bHOM model is examined for the simpler scenarios in more detail. This will be done for autoregulation en- and disabled, but without a swelling $\left(V_{E}=0 \mathrm{ml}\right)$. A closer look will be taken, how the volumes, pressures, resistances and compliances of interest behave in general.

\subsubsection{Case 1: no swelling, no autoregulation}

In the first scenario, the simulations were done with impaired autoregulation. Because of reduced complexity of the model, this makes the analysis of the system easier. Also, it is of general interest, how the system, as a whole, behaves without an enabled autoregulation.

\section{CBF}

One of the important properties of this model is its ability to calculate the cerebral blood flow $(\mathrm{CBF})$ of the system. As mentioned in chapter 3, the $\mathrm{CBF}$ is needed to simulate the oxygen transport from the capillaries to the brain tissue. Therefore, understanding the underlying mechanisms, which are responsible for the behavior of the $\mathrm{CBF}$, is crucial.

Figure 5.1 shows the CBF for the bHOM model with disabled autoregulation and without a swelling. The graph can be characterized as monoton- 


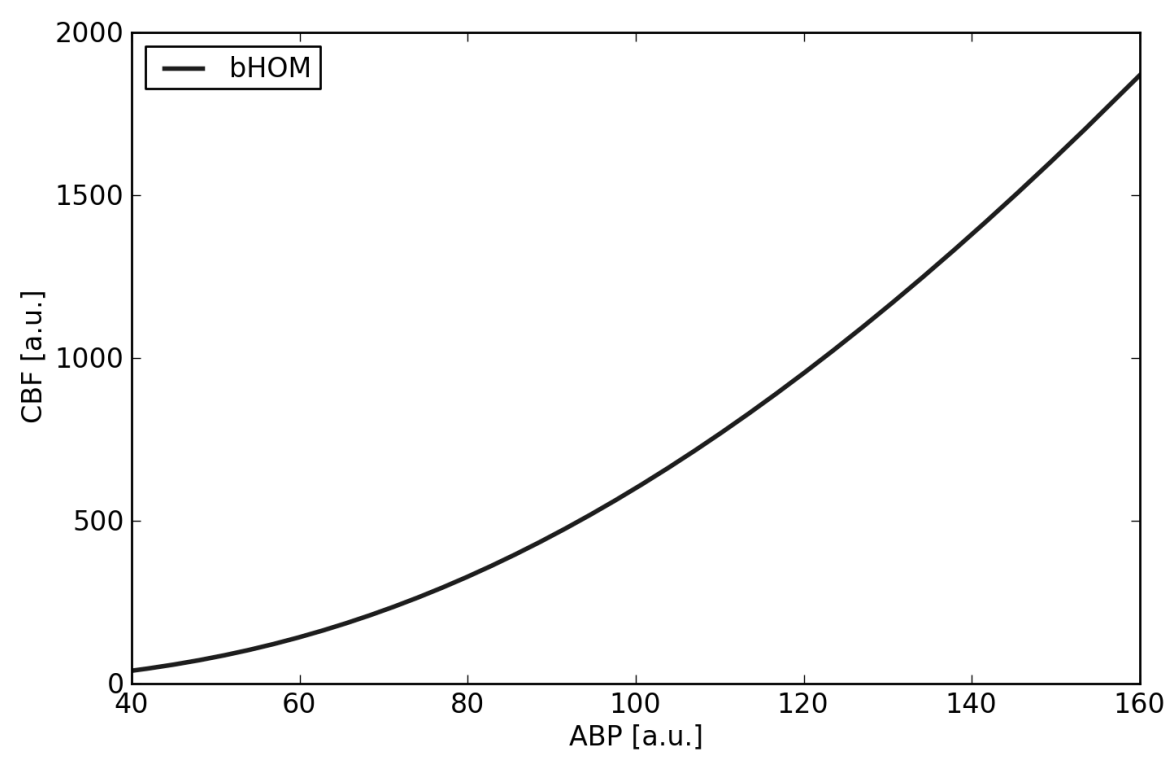

Figure 5.1: $\mathrm{CBF}$ against $\mathrm{ABP}$ with $\alpha=0.0$ and $V_{E}=0 \mathrm{ml}$ (bHOM; case $1)$.

ically increasing, whereas the curve gets flatter for values below $100 \mathrm{mmHg}$ and steeper for values above $100 \mathrm{mmHg}$. For understanding its general form, in the following sections a closer look on the underlying processes will be taken.

\section{Pressure}

As the the system is driven by the $\mathrm{ABP}$, at first the pressures inside the compartments are examined. In fig. 5.2 the time evolution of the pressure inside each compartment is shown. The pressure of the cerebrospinal fluid (CSF) compartment is not plotted, as it is assumed to be equal to the pressure inside the brain tissue (compare [32] and section 4.1.2).

It is obvious, that the pressure inside each compartment in general follows the development of the ABP. In the beginning (segment 1), the pressure in each compartment increases with the increasing ABP, until the ABP reaches its maximum at the end of segment 1 . From there, the pressure in each compartment decreases with the decreasing ABP (segment 2 and 3), until it reaches its minimum at the end of segment 3 . Thereafter, each pressure increases, until its standard value is reached again at the end of segment 4. The whole cycle repeats itself, due to the periodicity of the ABP.

In figure 5.3, the pressures inside each compartment are plotted against 


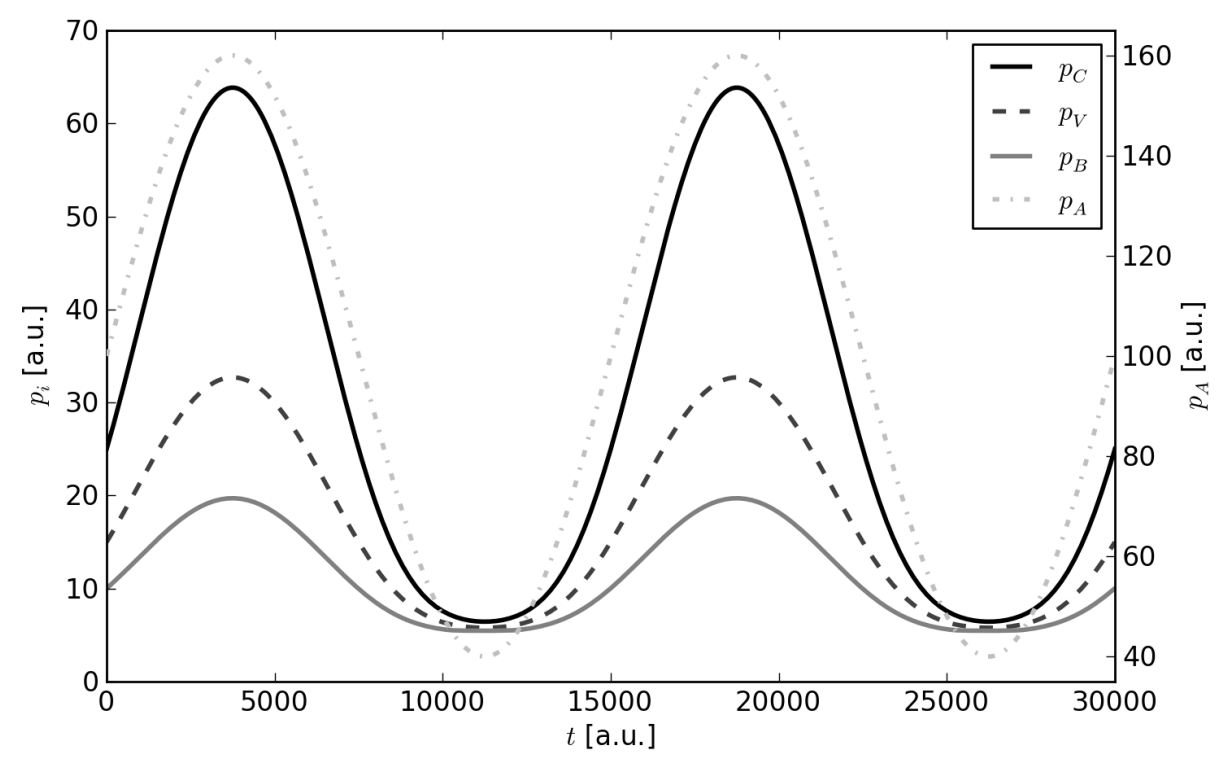

Figure 5.2: $p_{i}$ against time with $\alpha=0.0$ and $V_{E}=0 \mathrm{ml}$ (bHOM; case 1).

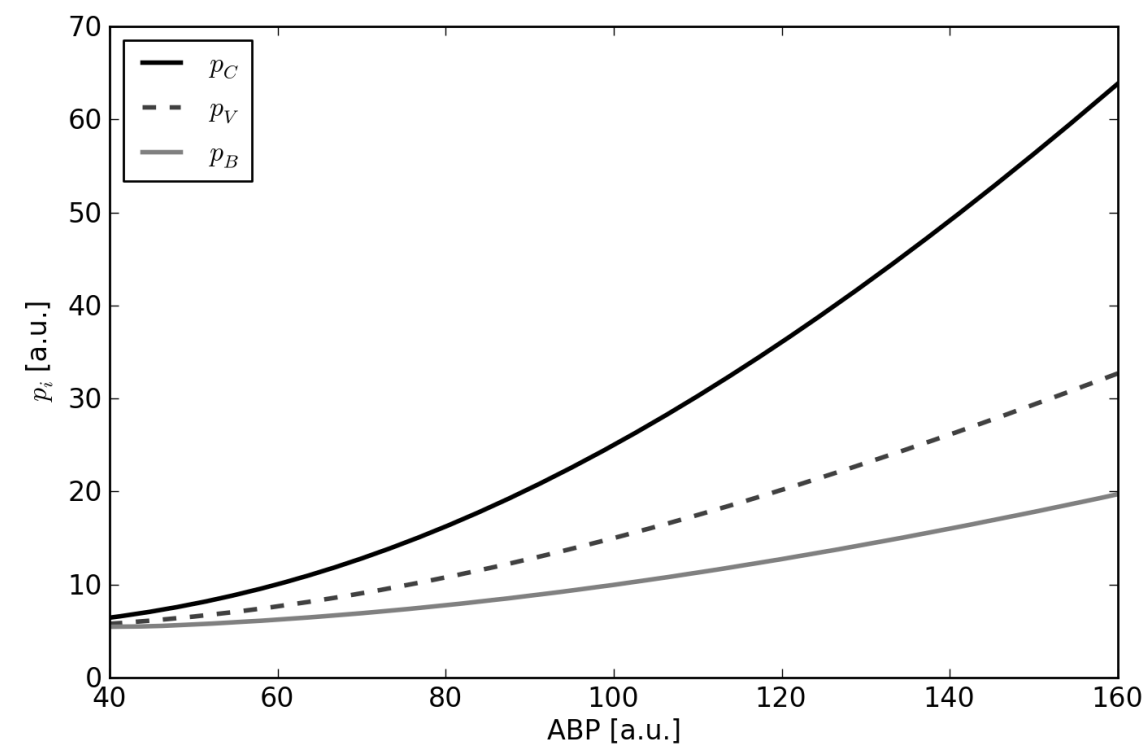

Figure 5.3: $p_{i}$ against ABP with $\alpha=0.0$ and $V_{E}=0 \mathrm{ml}$ (bHOM; case 1). 
the $\mathrm{ABP}$, whereas $p_{A}$ is not plotted, because it equals the ABP. In this form, the differences between the pressures inside each compartment are better reflected. As can be seen, the run of the curves for the pressures has an equal behavior, except for the ABP, which in this graph would be linearly increasing. Furthermore, the pressure gradient inside the blood vessels mentioned in section 4.1.2 can obviously be reproduced in a qualitative way: the pressure inside the arteries is always higher than inside the capillaries, which is bigger than the pressure inside the veins. Here, the intracranial pressure (ICP) is always below the pressure of the depicted blood vessels.

For decreasing pressures below $100 \mathrm{mmHg}$, all pressure curves of figure 5.3 seem to converge at around $5 \mathrm{mmHg}$. This is due to the fact, that the pressure inside the sagittal sinus, which is not depicted, is fixed at $5 \mathrm{mmHg}$. This marks the lowest possible pressure inside the system. Using ABP pressure values below $5 \mathrm{mmHg}$ for simulations does not make sense, as the blood flow would be reverted, which is not possible.

Table 5.1 summarizes the important data points, which are at the maximum, minimum and the default value for $\mathrm{ABP}$, and its range.

\begin{tabular}{l|r|r|r|r} 
Pressure $[\mathrm{mmHg}]$ & $\min$ & default & $\max$ & range \\
\hline$p_{A}$ & 40.00 & 100.00 & 160.00 & 120.00 \\
$p_{C}$ & 6.44 & 25.00 & 63.85 & 57.41 \\
$p_{V}$ & 5.78 & 15.00 & 32.70 & 26.92 \\
$p_{B}$ & 5.47 & 10.00 & 19.71 & 12.24
\end{tabular}

Table 5.1: Ranges for pressures with $\alpha=0.0$ and $V_{E}=0 \mathrm{ml}$ (bHOM; case $1)$.

\section{Compliances}

To better understand the nature of the volume changes, a closer look on the corresponding compliances is taken. As mentioned in chapter 3, the compliance of a compartment describes the development of the elastic membrane between two compartments and therefore, is a crucial part in the volume development.

For the model, the relevant compliances are $C_{A B}, C_{V B}$ and $C_{B}$. Figure 5.4 shows the graphs of these compliances, plotted in the time domain, whereas in figure 5.5 the graphs are plotted against ABP.

As the simulations are done with $\alpha=0, C_{A B}$ has to remain constant (compare eq. 3.7), as there is no autoregulation. It should be mentioned, that a constant compliance does not mean, that the volume of the corresponding compartment does not change, which can be deduced from eq. 3.3. Therefore, although the compliance for $C_{A B}$ is constant, the volume of the 


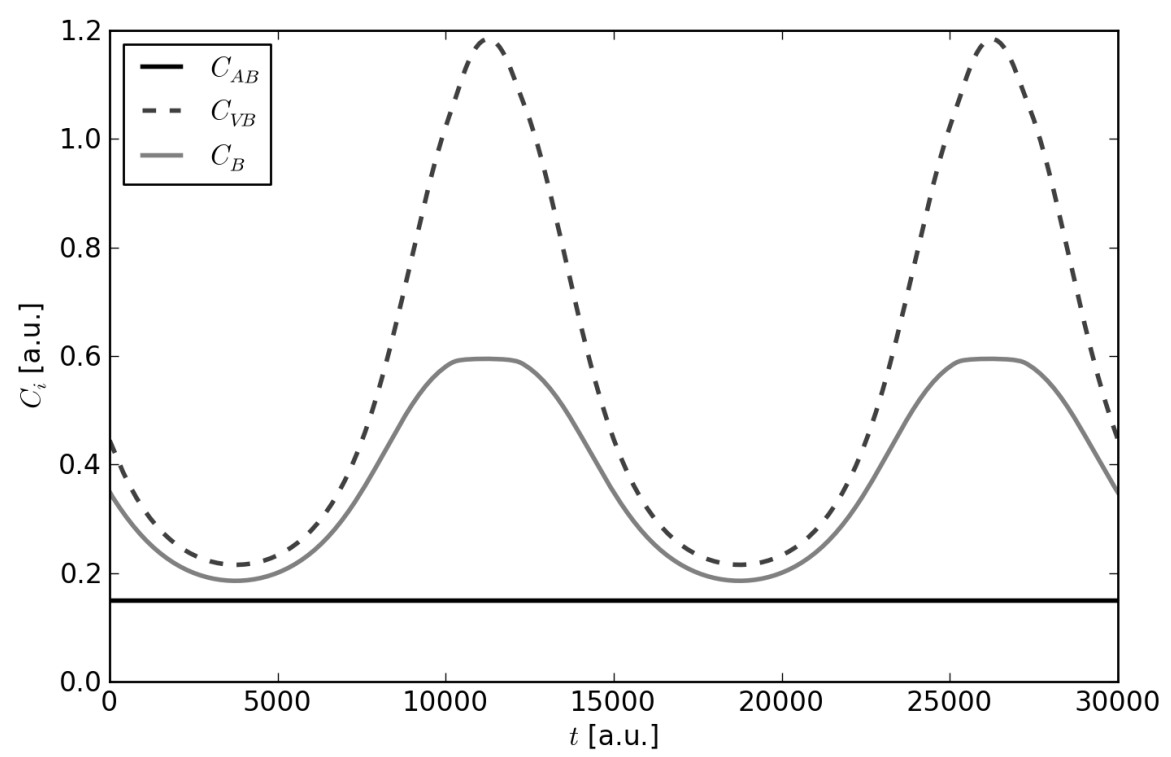

Figure 5.4: $C_{i}$ against time with $\alpha=0.0$ and $V_{E}=0 \mathrm{ml}$ (bHOM; case 1).

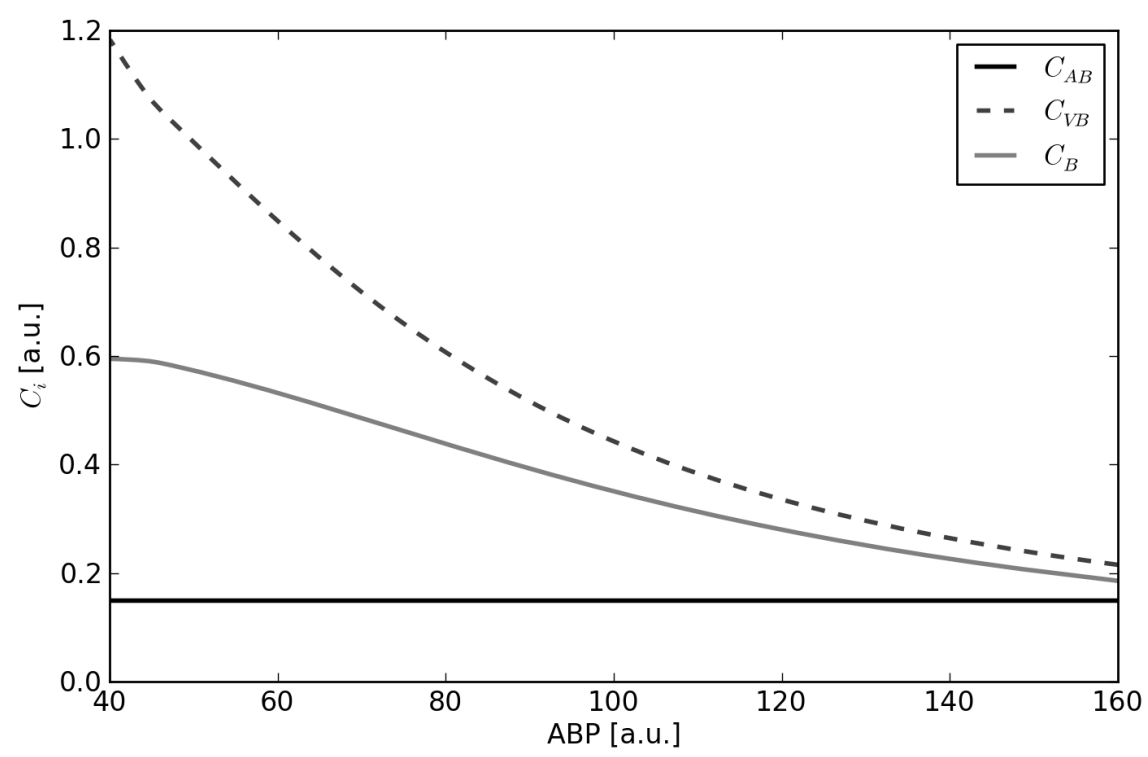

Figure 5.5: $C_{i}$ against ABP with $\alpha=0.0$ and $V_{E}=0 \mathrm{ml}$ (bHOM; case 1). 
arteries will change, depending on the pressure difference between the arteries and the brain tissue (compare with the following section about volumes).

To better understand the behavior of the other two compliances, the following formula, which describes the compliance of the brain tissue and veins, is useful:

$$
C=\frac{1}{k\left(|\Delta p|+p_{0}\right)}
$$

where $k$ is a constant and $p_{0}$ an offset, to avoid a singularity in case of $\Delta p=0$. Depending on the compliance, $\Delta p$ is the pressure difference between pressure inside the veins and brain tissue $\left(C_{V B}\right)$, and equals ICP $\left(C_{B}\right)$, respectively. For an $\mathrm{ABP}$ above about $120 \mathrm{mmHg}$, both graphs of $C_{V B}$ and $C_{B}$ have a similar slope with a small offset between them. This suggests, that in this range the pressure difference $p_{V}-p_{B}$ is correlated to $p_{B}$. For smaller $\mathrm{ABP}$ values, the graphs differ significantly. Whereas $C_{V B}$ increases more than linearly for decreasing $\mathrm{ABP}$, due to monotonic falling pressure difference $\Delta p$, the compliance $C_{B}$ increases less than linearly for decreasing $\mathrm{ABP}$ and flattens for an $\mathrm{ABP}$ of approx. $46 \mathrm{mmHg}$, from where on it becomes nearly constant. The reason for this behavior is the development of the ICP, which converges for smaller ABP values to a value of $5 \mathrm{mmHg}$. This suggests, that the change in volume of the brain tissue should also become constant below an ABP of $46 \mathrm{mmHg}$. On the contrary, the volume of the veins should decrease, although the pressure inside the veins converges to a value of $5 \mathrm{mmHg}$, due to the gradient of $C_{V B}$.

Table 5.2 summarizes the values for important ABP data points (minimum and maximum of $\mathrm{ABP}$ and their default values).

\begin{tabular}{l|r|r|r|r} 
Compliance $\left[\frac{\mathrm{ml}}{\mathrm{mmHg}}\right]$ & $\min$ & default & $\max$ & range \\
\hline$C_{A B}$ & 0.15 & 0.15 & 0.15 & 0.00 \\
$C_{V B}$ & 0.22 & 0.44 & 1.18 & 0.96 \\
$C_{B}$ & 0.19 & 0.35 & 0.59 & 0.40
\end{tabular}

Table 5.2: Ranges for compliances with $\alpha=0.0$ and $V_{E}=0 \mathrm{ml}$ (bHOM; case 1$)$.

\section{Resistances}

For the resistances only the graphs for the resistance of the arteries $R_{A C}$ and for the veins $R_{V S}$ are of interest. By definition, the resistance of $R_{C V}$ is constant. The involved resistances of the CSF, namely the resistance $R_{C F}$ of the inflow and $R_{F S}$ for the outflow, are also constant, as in this scenario the corresponding pressure differences are and the amount of CSF is always positive (compare eq. 3.16 and 3.17). The graphs are depicted in figure 5.6 in the time domain and in figure 5.7 against $\mathrm{ABP}$, respectively. 


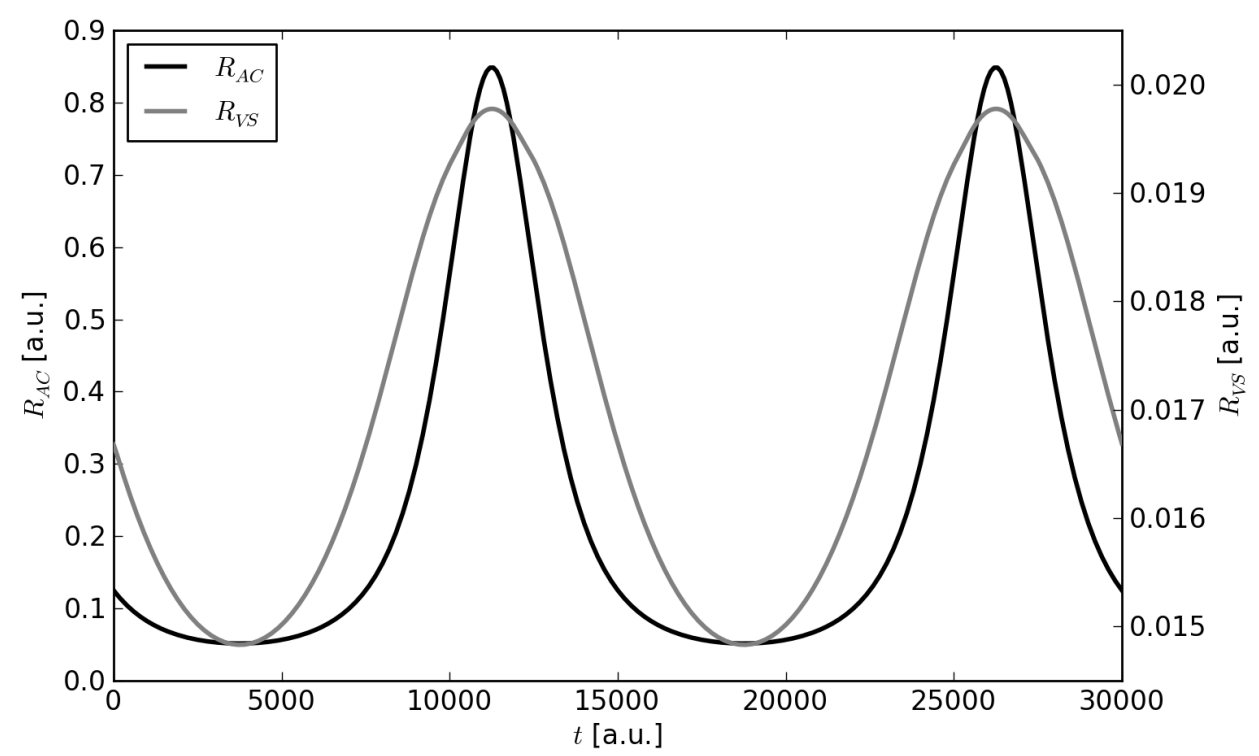

Figure 5.6: $R_{X Y}$ against time with $\alpha=0.0$ and $V_{E}=0 \mathrm{ml}$ (bHOM; case 1).

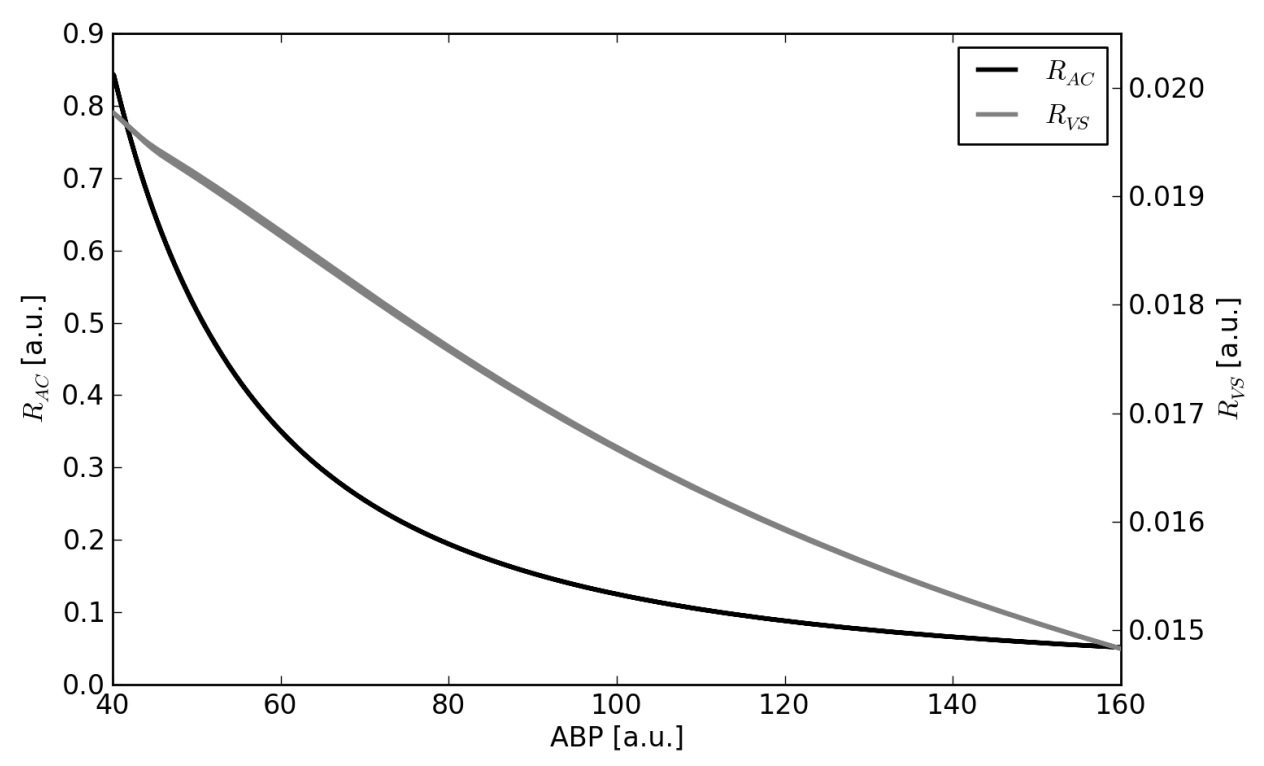

Figure 5.7: $R_{X Y}$ against $\mathrm{ABP}$ with $\alpha=0.0$ and $V_{E}=0 \mathrm{ml}$ (bHOM; case $1)$. 
The trend of $R_{A C}$ contains no surprises. For the maximum of ABP the resistance has the smallest value as, due to the highest pressure difference between arteries and brain tissue, the volume of the arteries has its maximum. For decreasing ABP their volume decreases and with it the resistance increases, until it hits its maximum for the smallest ABP and ICP difference, due to the smallest diameter, which corresponds to the smallest $\mathrm{ABP}$. In a first order approximation, the graph is proportional to $1 / \mathrm{ABP}^{2}$ (compare eq. 3.6; here, $C_{A B}$ is constant and $\mathrm{ABP} \gg \mathrm{ICP}$ ), which suggests a linear increase of the volume of the arteries with increasing ABP below $100 \mathrm{mmHg}$. But due to the smaller pressure difference between ABP and ICP for smaller ABP values, the deviation from a linear behavior should become more dominant for decreasing $\mathrm{ABP}$ values.

As for the trend of $R_{V S}$ it can be approximated by a linearly decreasing function. Here, it is obvious, that the arteries and veins are modeled differently, due to their different behavior in their resistances. Contrary to $R_{A C}$, no deduction about the development of the corresponding volume can be made, as the volume of the veins depends on $C_{V B}$, which is not constant. Also the volume $V_{V}$ depends on the pressure difference between the veins and brain tissue, not on the ABP.

Now, together with the previous sections, the development of the volume of the compartments can be explained and its behavior understood. A closer look on how the volumes develop in general will be taken in a following section.

\section{Volumes}

An interesting aspect of the model is the possibility, to see, how the volume of each compartment develops. In order to better compare the volume changes of each compartment, here, the volumes minus the standard value for the corresponding volume are plotted and will be labeled with $\Delta V_{i}$. This allows a side by side depiction of the curves, as the changes in volume are in a comparable range, but the absolute values differ in the range of two orders of magnitude (compare section 4.1.1).

In figure 5.8 the change in volume $\Delta V_{i}$ of each compartment against time is shown. For discussion, the graphs are separated into four segments (as described in section 5.1).

In the first segment, due to a raising ABP, the pressure inside the arteries and therefore, the pressure inside the veins as well, increases. As in this scenario the change of volume for the arteries only depends on their compliance, which is constant, and on the pressure difference between ABP and ICP, which increases, the volume of the arteries has to increase. The volume of the veins has to increase as well, as the inflow into the vein compartment increases faster than its outflow. This is due to the fact, that $p_{C}-p_{V}$, as $R_{C V}$ remains constant, increases much faster than $p_{V}-p_{S}$ (compare fig. 5.3 


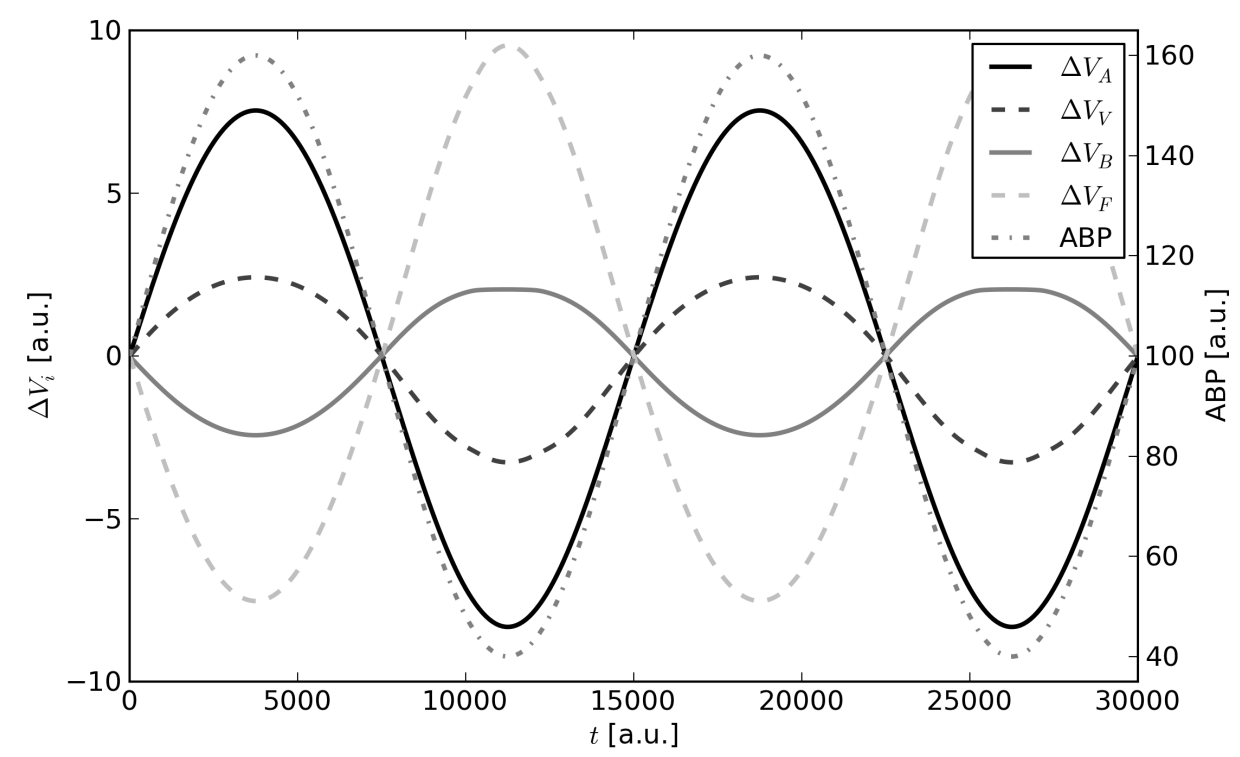

Figure 5.8: $\Delta V_{i}$ against time with $\alpha=0.0$ and $V_{E}=0 \mathrm{ml}$ (bHOM; case 1).

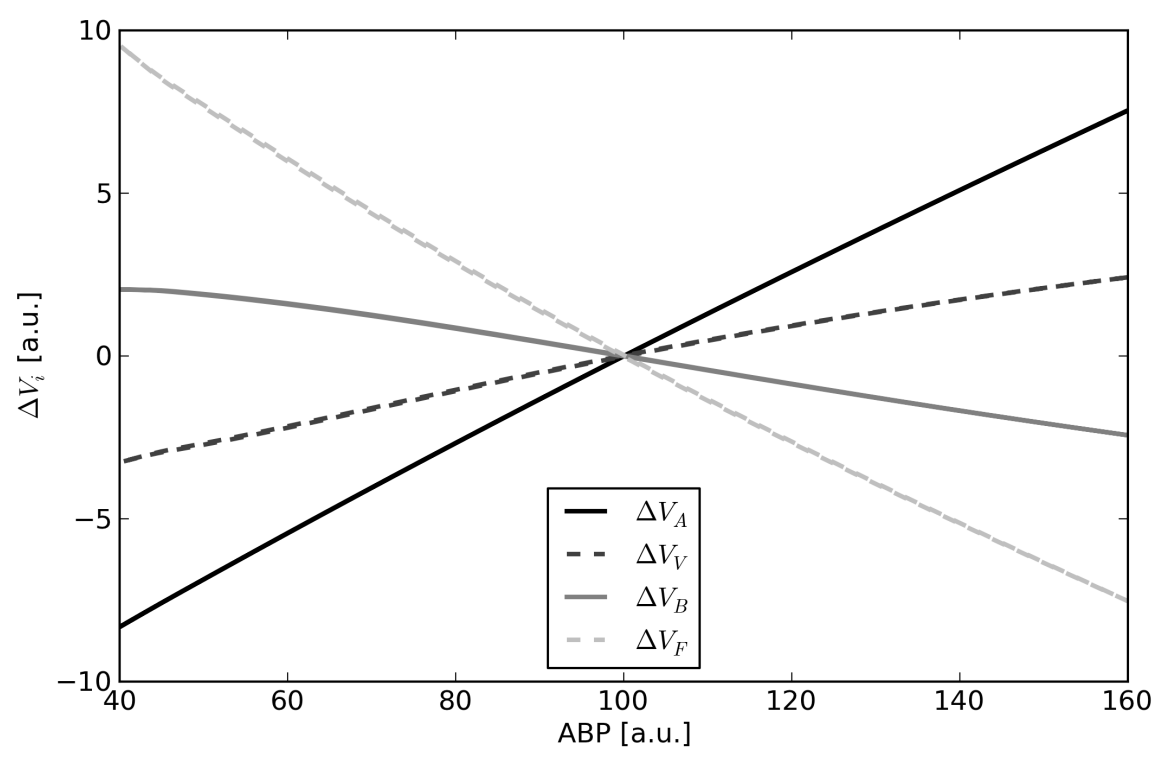

Figure 5.9: $\Delta V_{i}$ against ABP with $\alpha=0.0$ and $V_{E}=0 \mathrm{ml}$ (bHOM; case 1). 
and table 5.1). The decrease in $R_{V S}$, which enhances the increased outflow of the vein compartment, is too small, to compensate this.

The volume of the brain tissue decreases, as does the volume of the CSF compartment. The process is reverted in segment two. In the third and forth segment, the process happens in an analog way: Due to the falling ABP in the third segment, the volume of the arteries and veins decreases, and the volume of the brain tissue and CSF increases. This process is reverted in the forth segment. The change in volume is linear in a first approximation, as can be seen in fig. 5.9, which shows $\Delta V_{i}$ against ABP. This was suggested in the previous sections for the arteries, but holds in a first approximation for all volume changes.

An interesting result is, that for an $\mathrm{ABP}$ higher than $100 \mathrm{mmHg}$, the change in volume of the veins is nearly identical to the reverse change of volume of the brain tissue (compare table 5.3). An analog result can be drawn for the volume change of the arteries and the CSF.

For an ABP lower than $100 \mathrm{mmHg}$ the results differ slightly. The graphs for the volume change of the arteries and veins are still nearly linear, but the volume change of CSF and brain tissue is not. As the pressure inside the brain converges for small ABP values, as well as the compliance of the brain tissue, the change in volume also has to become constant, which can be seen in the graph. On the contrary, the change of CSF raises in order to compensate this behavior of the brain tissue.

Another difference lies in a steeper negative change for the veins and arteries for an ABP below $100 \mathrm{mmHg}$ than for the positive change for an ABP above $100 \mathrm{mmHg}$. This can directly be seen in table 5.3, where the important values for the maximal and minimal volume changes are summarized.

\begin{tabular}{l|r|r|r}
$\Delta$ Volume $[\mathrm{ml}]$ & $\min$ & $\max$ & range \\
\hline$\Delta V_{A}$ & -8.32 & 7.54 & 15.86 \\
$\Delta V_{B}$ & -2.43 & 2.04 & 4.47 \\
$\Delta V_{F}$ & -7.53 & 9.54 & 17.07 \\
$\Delta V_{V}$ & -3.27 & 2.42 & 5.69
\end{tabular}

Table 5.3: Ranges for volume changes with $\alpha=0.0$ and $V_{E}=0 \mathrm{ml}$ (bHOM; case 1).

In case of the arteries, due to the constant compliance, the reason lies in the faster decreasing pressure difference between $\mathrm{ABP}\left(p_{A}\right)$ and $\operatorname{ICP}\left(p_{B}\right)$, which can directly be deduced from eq. 3.3. In case of the veins, the reason lies in the development of the in- and outflow of the venous compartment. With decreasing ABP below $100 \mathrm{mmHg}$, the inflow into the venous compartment decreases faster than the outflow. As the pressure difference between the capillaries and veins is getting more similar with decreasing ABP 
(compare fig. 5.3), due to the assumed constant resistance of the capillaries the change of the inflow decreases more or less linearly. But the outflow decreases less than linearly: Though the resistance of the veins increases linearly (compare fig. 5.7), the pressure difference between the veins and sagital sinus decreases less than linearly, due to the assumed constant pressure inside the sinus. The difference is compensated by the CSF production, which results in a higher value of the CSF production in segment three and four, than in the CSF absorption in the first and second segment.

For $\Delta V_{i}$, only a qualitative description can be done. Another view on the development of the volume can be achieved, if the deviation of the volume is used, which gives a more quantitative description. Figure 5.10 shows $\dot{V}_{i}$ of the different compartments in the time domain, whereas in figure 5.11 they are depicted against ABP.

The aforementioned correlation between the volume change of the arteries and CSF, and the volume change of the brain tissue and veins can be seen in figure 5.11. For ABP values above $100 \mathrm{mmHg}$ the change in volume is nearly inversely identical in the corresponding compartments (the corresponding deviations of the volumes are anti-correlated, which can be seen in figure 5.10), whereas for smaller values, the difference between $\dot{V}_{B}$ and $\dot{V}_{V}$ is compensated by the CSF. Also, the stagnation of the volume change of the brain tissue is better reflected in this depiction.

But there seems to be a problem with the behavior of the veins and the brain tissue volume at an ABP of approximately $47 \mathrm{mmHg}$. There are bumps in the graphs and in the case of $\dot{V}_{V}$, the graph has even a nonmonotonic slope. From a medical point of view, there is no reason, why such a disturbance should occur. This indicates, that a modification to the model is needed, although in general, the model behaves as expected.

\subsubsection{Case 2: no swelling, autoregulation}

After having analyzed the behavior of the model without autoregulation in the previous section, here, the simulations will be repeated with an enabled autoregulation mechanism. The parameters for the autoregulation mechanism, used in the simulations, are the values given in chapter 4 .

From a medical point of view this represents the normal conditions inside the human brain: autoregulation on and no swelling. To get a better understanding of the underlying processes involved in the general haemodynamics of the human brain a highly varying ABP is used, to see, how the system behaves inside the limits of the autoregulation mechanism and outside.

\section{CBF}

As before, first, we will take a look at the $\mathrm{CBF}$ against $\mathrm{ABP}$, which is pictured in figure 5.12 for enabled autoregulation $(\alpha=2)$, together with the 


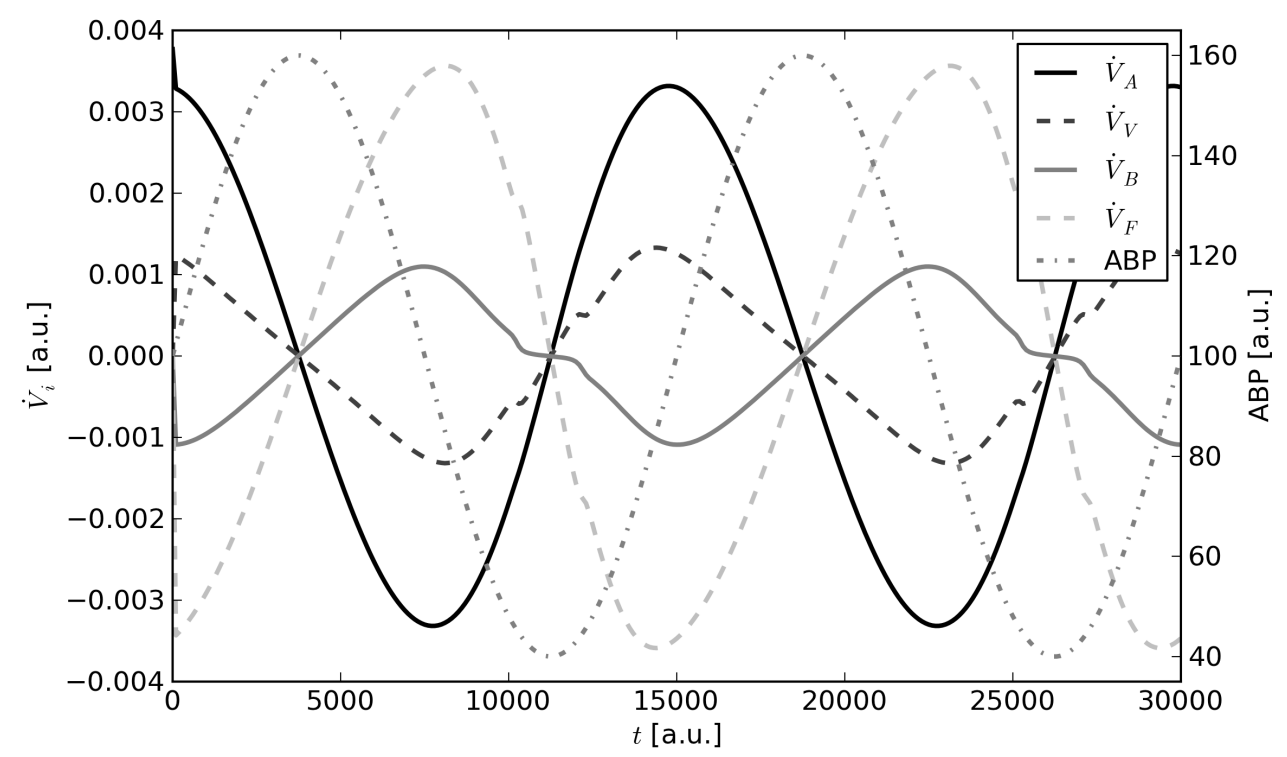

Figure 5.10: $\dot{V}_{i}$ against time with $\alpha=0.0$ and $V_{E}=0 \mathrm{ml}$ (bHOM; case 1 ).

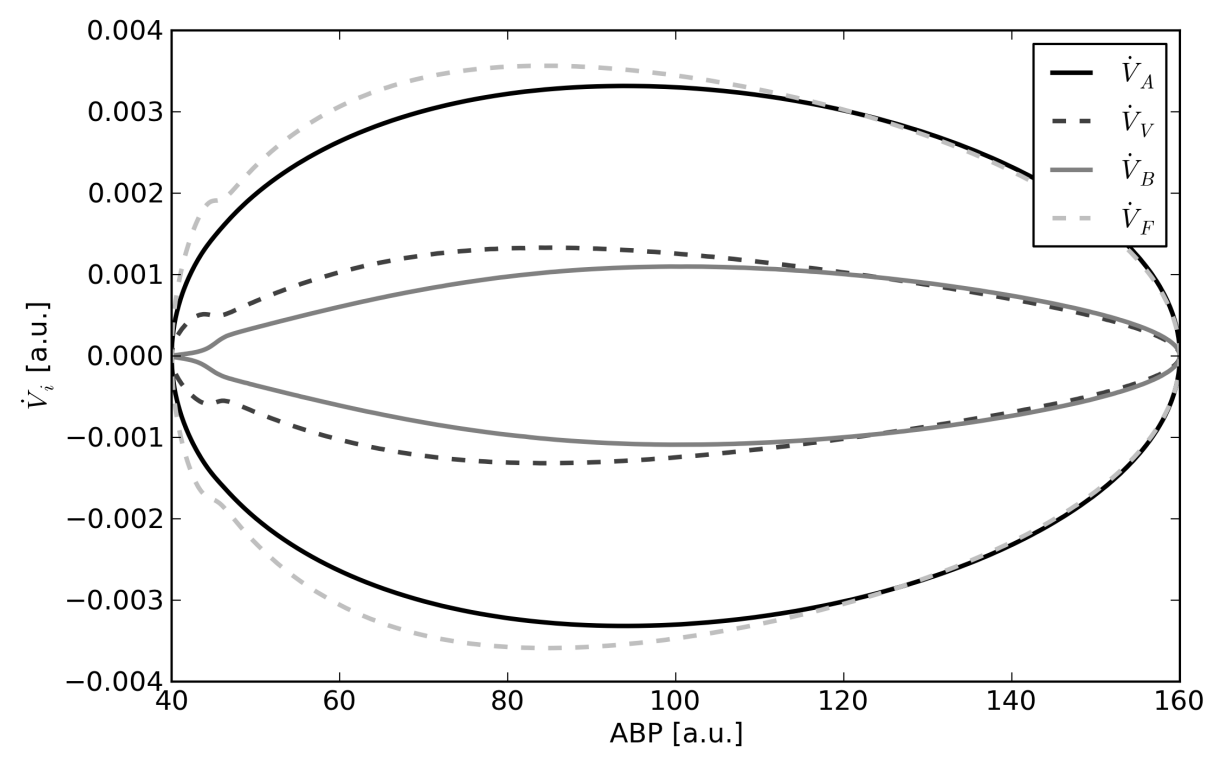

Figure 5.11: $\dot{V}_{i}$ against ABP with $\alpha=0.0$ and $V_{E}=0 \mathrm{ml}$ (bHOM; case 1). 


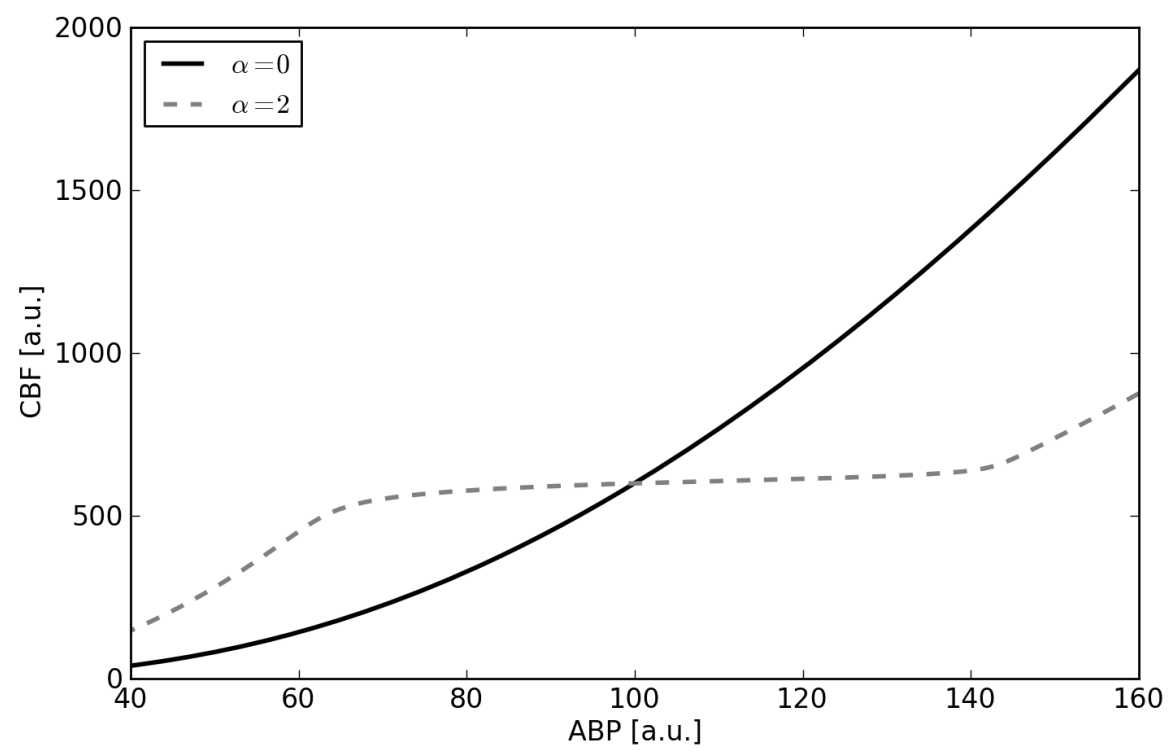

Figure 5.12: CBF against ABP for disabled $(\alpha=0.0)$ and enabled $(\alpha=2.0)$ autoregulation and no swelling (bHOM; case 2$)$.

CBF of the previous case for disabled autoregulation $(\alpha=0)$.

If the CBF of the simulations with and without autoregulation are compared directly, amongst others the ranges of CBF for both scenarios differ. For a functioning autoregulation, the $\mathrm{CBF}$ reaches values for the highest $\mathrm{ABP}$ values, which are only half as big. For the lowest $\mathrm{ABP}$ values, the $\mathrm{CBF}$ is still above $150 \mathrm{ml} / \mathrm{min}$ and raising fast for increasing $\mathrm{ABP}$ values, until a plateau is reached, whereas for a disabled autoregulation CBF values above $150 \mathrm{ml} / \mathrm{min}$ are reached not until an ABP of approximately $60 \mathrm{mmHg}$.

But the most obvious difference lies in the plateau between an $\mathrm{ABP}$ of about $60 \mathrm{mmHg}$ and $140 \mathrm{mmHg}$. As already seen in section 4.5.1, this plateau has developed due to the autoregulation mechanism. But now a more in-depth view for the course of the graphs will be made by examining the underlying processes, to get a better understanding of how this plateau is achieved. Additionally, it will be examined, how the internal processes of the system are altered compared to the previous scenario with disabled autoregulation.

As the only difference between both simulations lies in the altering of the compliance $C_{A B}$, due to $\alpha \neq 0$, contrary to the former discussion the graphs of the compliance are examined first. 


\section{Compliances}

The only reason for the alteration of the CBF lies in the ability of the arteries to actively change their compliance, depending on the actual $\mathrm{CBF}$ value, as described in chapter 2 and 3. Therefore, it is not surprising, that the graphs for the compliances, shown in fig. 5.13 in the time domain and in fig. 5.14 against ABP, differ from the former scenario (compare with fig. 5.4 and 5.5).

At first, the compliance of the arteries is not constant anymore. As described in section 4.5.1 the compliance is altered in such a way, that the actual $\mathrm{CBF}$ is as close as possible to its standard value. Furthermore, the limits of the autoregulation mechanism can easily be seen in fig. 5.14: For $\mathrm{ABP}$ values lower than $60 \mathrm{mmHg}$ the compliance is constant, as there is no possibility to actively further increase the diameter. With raising values for $\mathrm{ABP}$ the compliance decreases, due to reducing the diameter, until it reaches its minimum at an ABP value of approximately $140 \mathrm{mmHg}$. From here, the active part of the arteries is totally constricted. Equally to the previous case, the compliance remains constant from that point on for higher ABP values, but it should be mentioned, that the volume of the arteries still changes, due to the pressure difference between arteries and brain tissue. The graph also reflects the asymmetry of the autoregulation mechanism. For ABP values above $100 \mathrm{mmHg}$, the maximal change in $C_{A B}$ is smaller than for $\mathrm{ABP}$ values below (compare table 5.4), due to different $\Delta C_{A B}$.

\begin{tabular}{l|r|r|r|r} 
Compliance $\left[\frac{\mathrm{ml}}{\mathrm{mmHg}}\right]$ & $\min$ & default & $\max$ & range \\
\hline$C_{A B}$ & 0.085 & 0.150 & 0.315 & 0.230 \\
$C_{V B}$ & 0.355 & 0.444 & 0.845 & 0.490 \\
$C_{B}$ & 0.292 & 0.350 & 0.527 & 0.253
\end{tabular}

Table 5.4: Ranges for compliances with $\alpha=2.0$ and $V_{E}=0 \mathrm{ml}$ (bHOM; case 2$)$.

The shape of the compliances of the brain tissue and veins looks different compared to the arteries: For values between 60 and $140 \mathrm{mmHg}$, the regulation range of the arteries, the compliances $C_{V B}$ and $C_{B}$ remain approximately constant, whereas for higher values, both decrease $\left(C_{V B}\right.$ stronger than $C_{B}$ ) and for lower values than $60 \mathrm{mmHg}$ both increase (also, $C_{V B}$ stronger than $C_{B}$ ). As both $C_{V B}$ and $C_{B}$ are only pressure dependent (compare eq. 3.12 and 3.18), it can be deduced, that the pressure inside the brain and veins remain constant inside the autoregulation range, and follows the trend of the CBF development outside of the range. Due to the smaller ranges of the compliances (except $C_{A B}$ ), the variation of the pressure should also be smaller (compare next section about pressure), when compared to the previous case. 


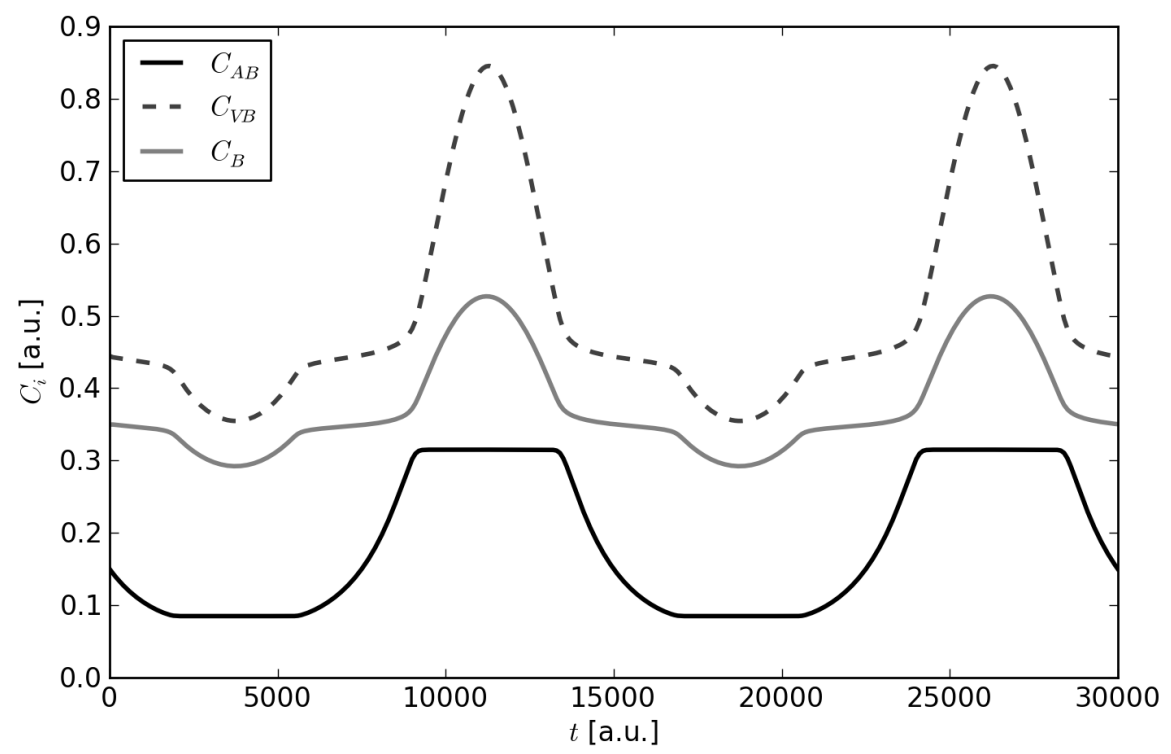

Figure 5.13: $C_{i}$ against time with $\alpha=2.0$ and $V_{E}=0 \mathrm{ml}$ (bHOM; case 2).

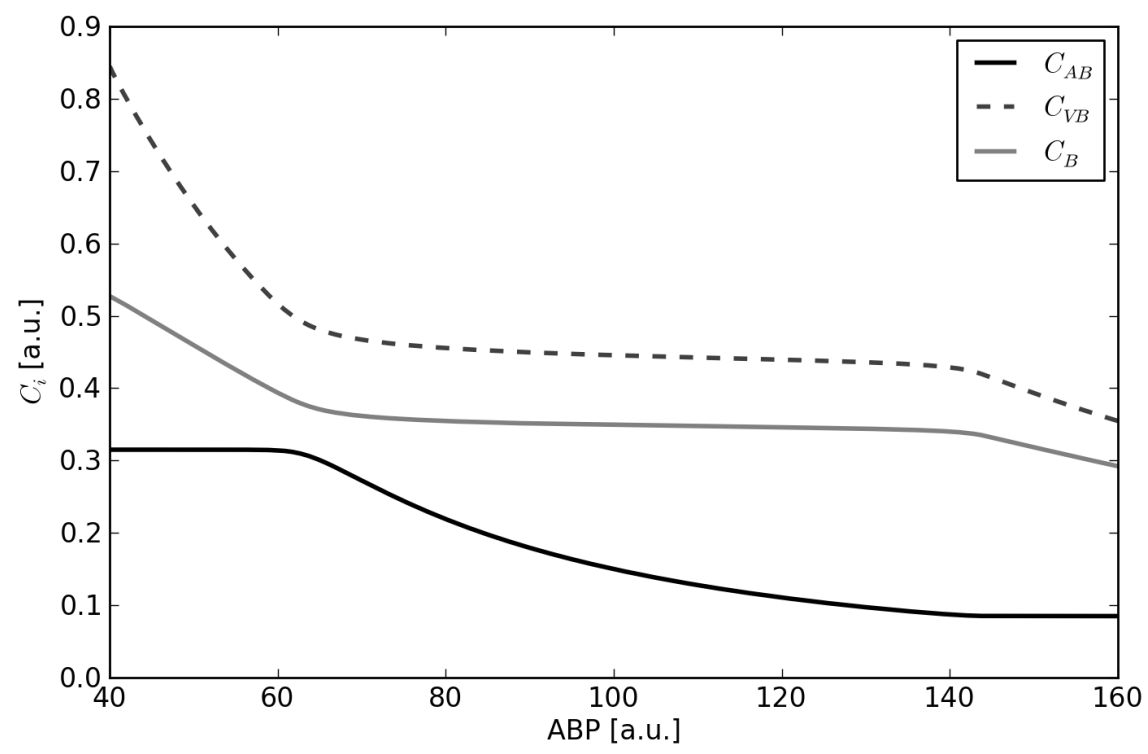

Figure 5.14: $C_{i}$ against $\mathrm{ABP}$ with $\alpha=2.0$ and $V_{E}=0 \mathrm{ml}$ (bHOM; case 2). 


\section{Pressure}

In fig. 5.15 and 5.16 the graphs for the pressures $p_{C}, p_{V}$ and $p_{B}$ are depicted. For the graphs in the time domain, $p_{A}$ is plotted additionally. As assumed in the previous section, the pressures have developed a plateau inside the range of the autoregulation mechanism and have in general a similar shape as the CBF (compare fig. 5.12). The average value of the plateau is around the corresponding default value.

Outside of the autoregulation the development of the pressures can be approximated by a linear increase/decrease. For decreasing ABP below $50 \mathrm{mmHg}$, the development of the graphs changes from its nearly linear behavior to a convergence to the value of $5 \mathrm{mmHg}$, the lowest possible value in the blood vessel system, as mentioned in the previous section.

When compared with the simulations done in case 1 , the range of the pressure inside each blood vessel type has more than halved, which can be seen in tables 5.1 and 5.5. Also, the minimal values of the pressures are higher than their corresponding pressures in case 1, in which the autoregulation is disabled. The reason for this increase stems from the active constriction of the arteries and thereby reducing their resistance, which results in a smaller reduced pressure gradient throughout the blood vessels. In an analog way the maximal value of the pressures are decreased, because of the dilation of the arteries.

\begin{tabular}{l|r|r|r|r} 
Pressure $[\mathrm{mmHg}]$ & $\min$ & default & $\max$ & range \\
\hline$p_{A}$ & 40.00 & 100.00 & 160.00 & 120.00 \\
$p_{C}$ & 10.20 & 25.00 & 33.66 & 23.46 \\
$p_{V}$ & 7.74 & 15.00 & 19.06 & 11.32 \\
$p_{B}$ & 6.30 & 10.00 & 12.16 & 5.86
\end{tabular}

Table 5.5: Pressure ranges with $\alpha=2.0$ and $V_{E}=0 \mathrm{ml}$ (bHOM; case 2).

The development of the corresponding resistances will be discussed in the next section.

\section{Resistances}

From the former sections it can be assumed, that in general there are two interesting domains: in- and outside of the autoregulation mechanism. For the interesting resistances, namely of the arteries $R_{A C}$ and veins $R_{V S}$, the graphs are shown in figures 5.17 (time domain) and 5.18 (against ABP).

With the findings, obtained in the previous sections about the compliances and pressures, the development of $R_{V S}$ can easily be explained: Due to the constant pressure and compliance between an $\mathrm{ABP}$ of about $60 \mathrm{mmHg}$ and $140 \mathrm{mmHg}$, the corresponding resistance has to be constant. Outside 


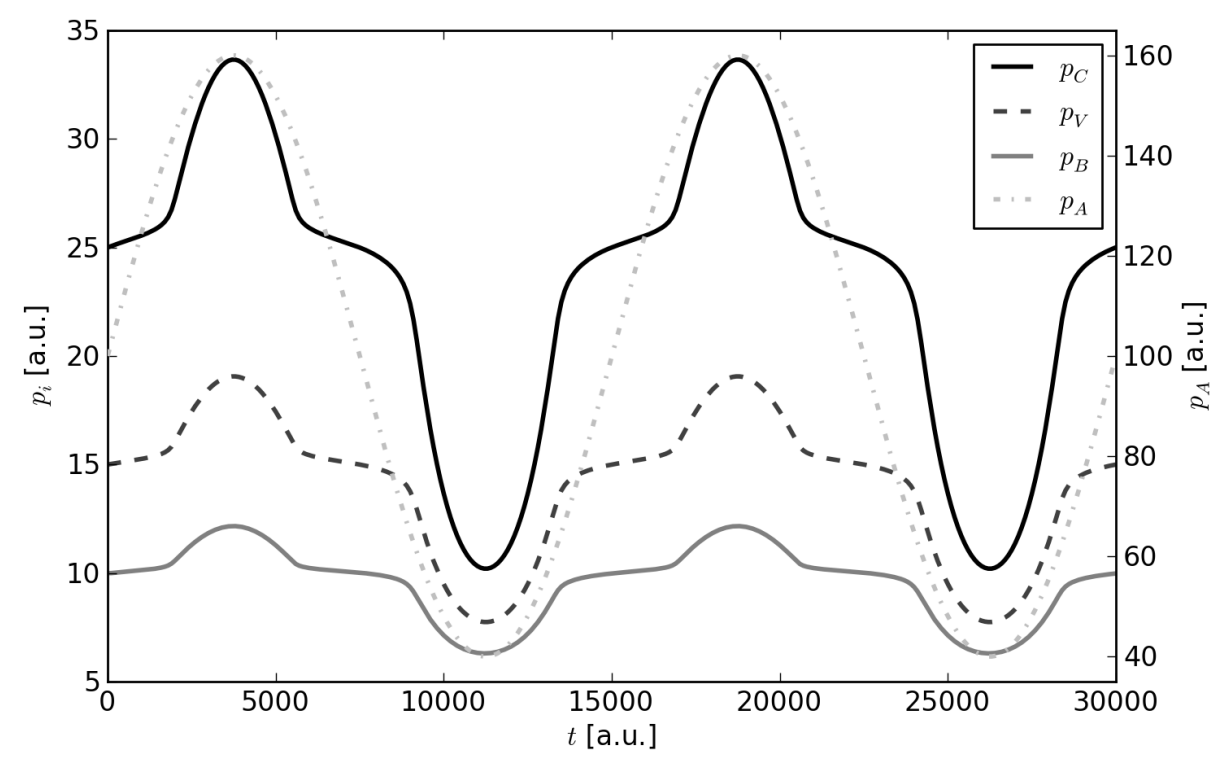

Figure 5.15: $p_{i}$ against time with $\alpha=2.0$ and $V_{E}=0 \mathrm{ml}$ (bHOM; case 2).

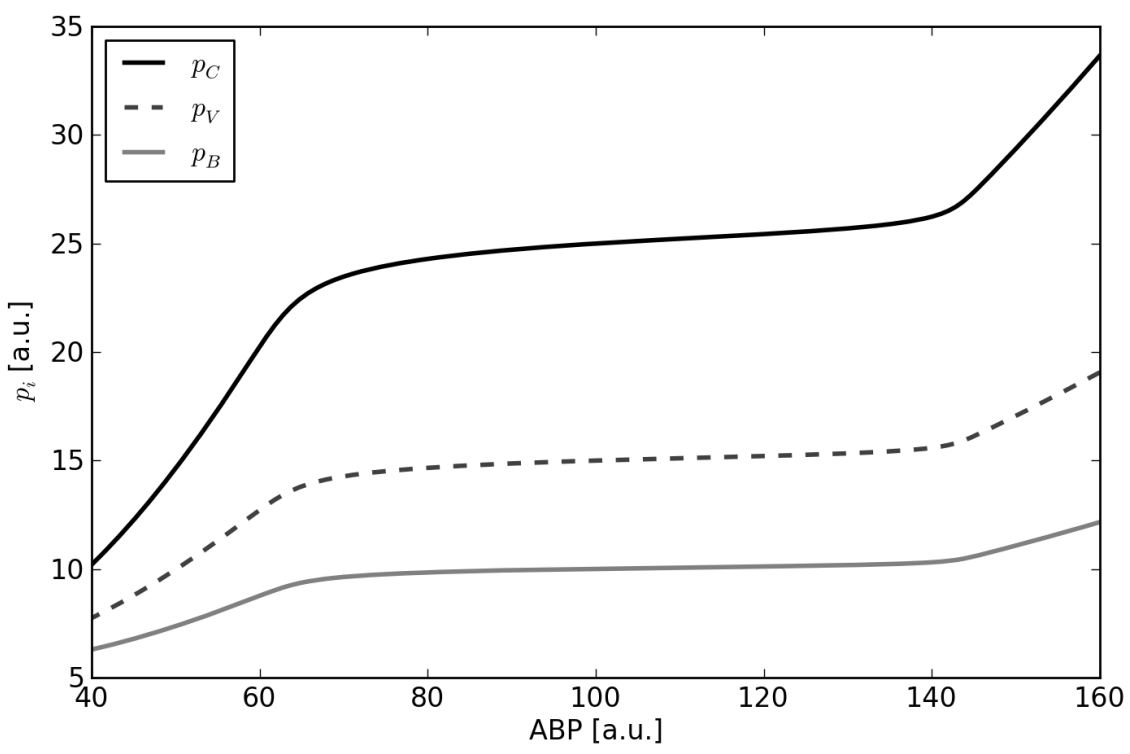

Figure 5.16: $p_{i}$ against ABP with $\alpha=2.0$ and $V_{E}=0 \mathrm{ml}$ (bHOM; case 2). 


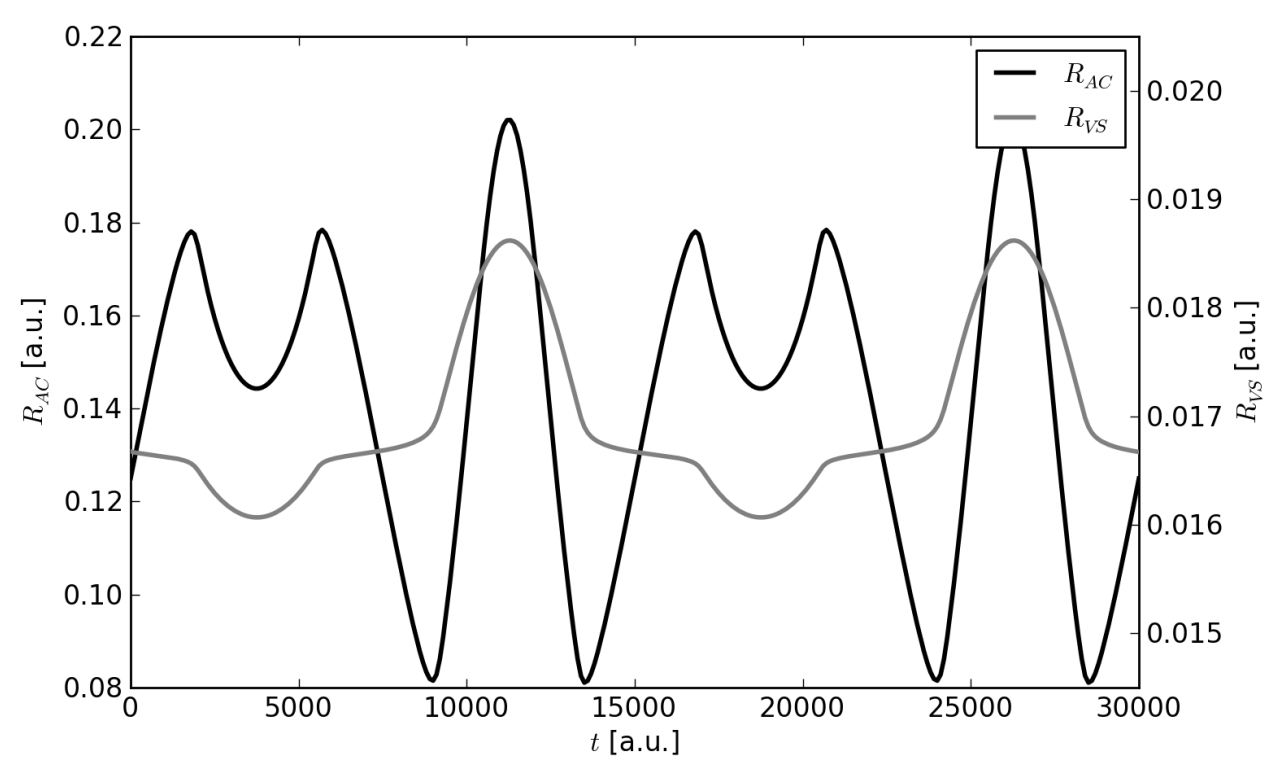

Figure 5.17: $R_{X Y}$ against time with $\alpha=2.0$ and $V_{E}=0 \mathrm{ml}$ (bHOM; case 2).

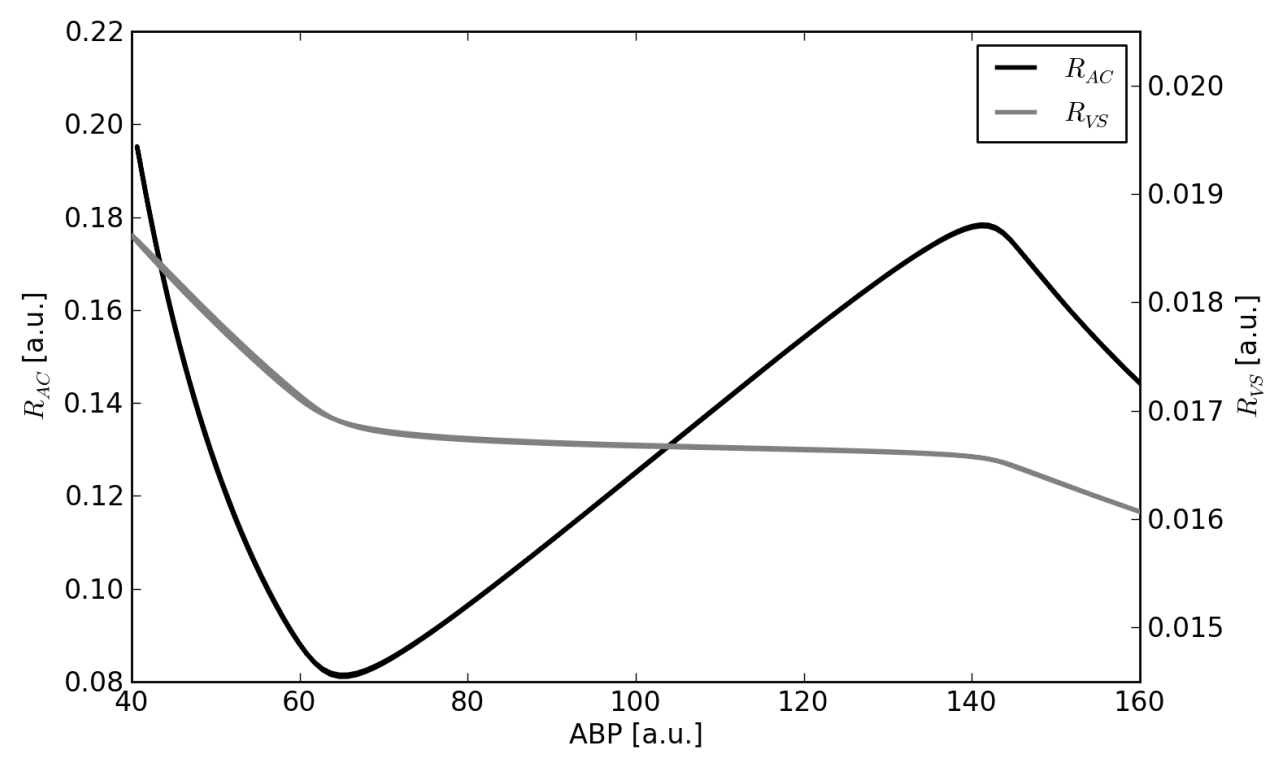

Figure 5.18: $R_{X Y}$ against $\mathrm{ABP}$ with $\alpha=2.0$ and $V_{E}=0 \mathrm{ml}$ (bHOM; case $2)$. 
this range, the resistance has to decrease with increasing ABP. As both, pressure and compliance, can be approximated by a linear function, the decrease in resistance has to be approximately linear, too.

The reason for the development of the resistance of the arteries is more complicated, due to the autoregulation mechanism: To reproduce a plateau in the CBF development, as seen in fig. 5.12, the resistance has to change in such a way, that the development of the pressure difference between arteries and capillaries is canceled out. As the pressure inside the capillaries is constant in this range, the form of the resistance of the arteries must follow the form of the ABP. This is exactly what can seen in fig. 5.18, where $R_{A C}$ develops nearly linearly inside the autoregulation range. For decreasing ABP values below $60 \mathrm{mmHg}, R_{A C}$ raises, as the volume of the arteries decreases, due to a constant compliance and decreasing pressure difference between $\mathrm{ABP}$ and ICP. For an increasing ABP above $140 \mathrm{mmHg}$, their compliance is constant, too. Due to an increase in pressure difference between ABP and ICP, the volume of the arteries increases, which results in a decrease of $R_{A C}$.

\section{Volumes}

Looking at the development of $\Delta V_{i}$ of each compartment in the time domain, the graphs shown in fig. 5.19 are much more complicated than for the former scenario, although only one aspect of the model has been changed. Plotting the volume changes against ABP (fig. 5.20), the picture becomes clearer, but is still more complex than before.

In this view, the effect of altering the compliance can easily be seen: Inside the autoregulation range, the volume of the arteries decreases with increasing $\mathrm{ABP}$, in order to maintain a constant $\mathrm{CBF}$ and therefore a constant supply of oxygen to the brain tissue. For decreasing ABP values below the regulation range, the volume decreases as the pressure drops, due to the constant compliance. The same happens in an analog way for increasing $\mathrm{ABP}$ values above. As the compliance for $\mathrm{ABP}$ below $60 \mathrm{mmHg}$ is higher than for a disabled autoregulation, the volume drop is steeper. On the contrary, the raise in volume above $140 \mathrm{mmHg} \mathrm{ABP}$ is flatter (compare fig. 5.9 and 5.20).

As before, the volume of the brain tissue decreases monotonically as the volume of the veins increases with the pressure inside themselves. The difference in the development of the volume for the brain tissue and veins in both scenarios lies mainly in the plateau: As the compliances and pressures are constant inside the autoregulation range, so are the corresponding volumes. The smaller range in volume exists mainly due to the smaller range in pressure.

When comparing the change of volume for brain tissue and veins, and for arteries and CSF, a similar result as in the former case is obtained: The 


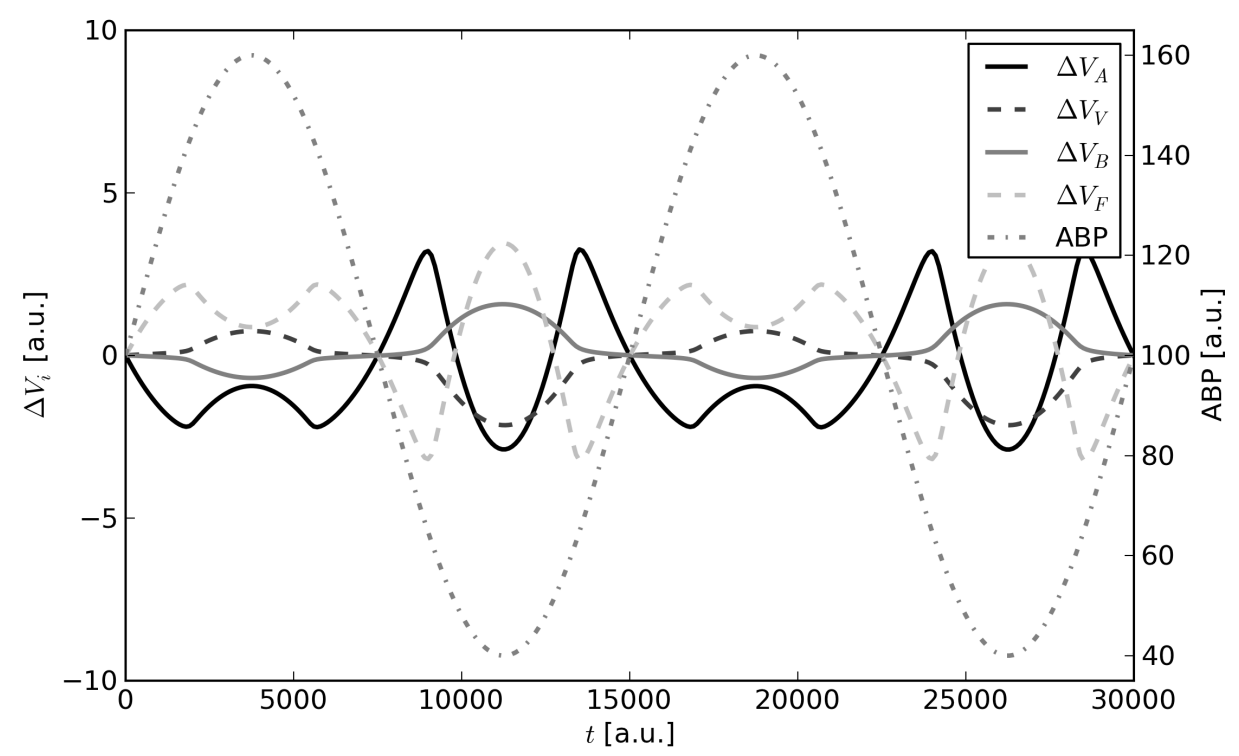

Figure 5.19: $\Delta V_{i}$ against time with $\alpha=2.0$ and $V_{E}=0 \mathrm{ml}$ (bHOM; case 2).

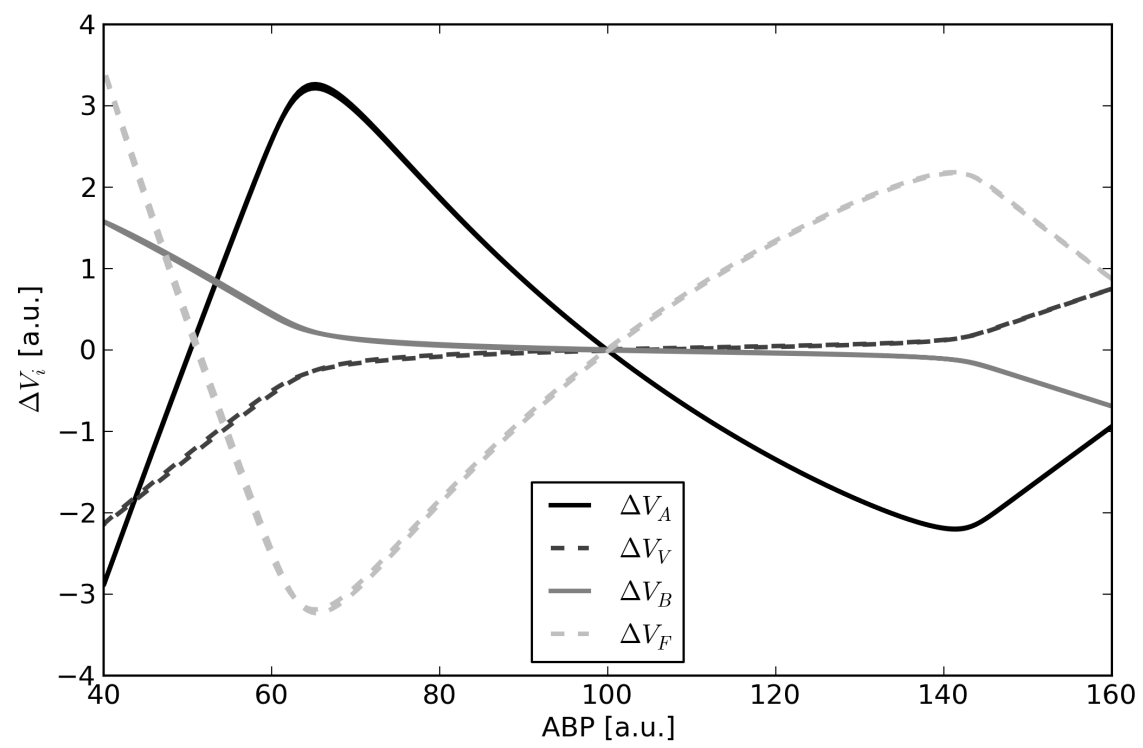

Figure 5.20: $\Delta V_{i}$ against $\mathrm{ABP}$ with $\alpha=2.0$ and $V_{E}=0 \mathrm{ml}$ (bHOM; case $2)$. 


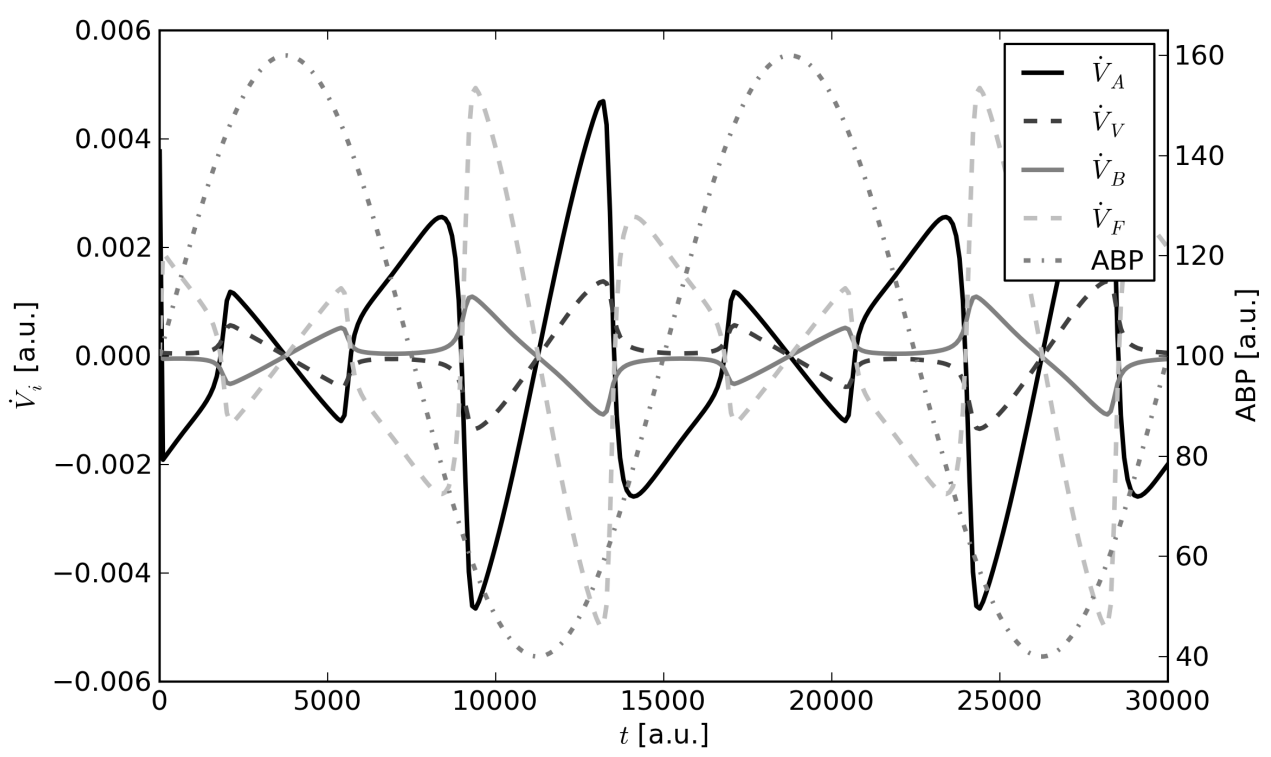

Figure 5.21: $\dot{V}_{i}$ against time with $\alpha=2.0$ and $V_{E}=0 \mathrm{ml}$ (bHOM; case 2).

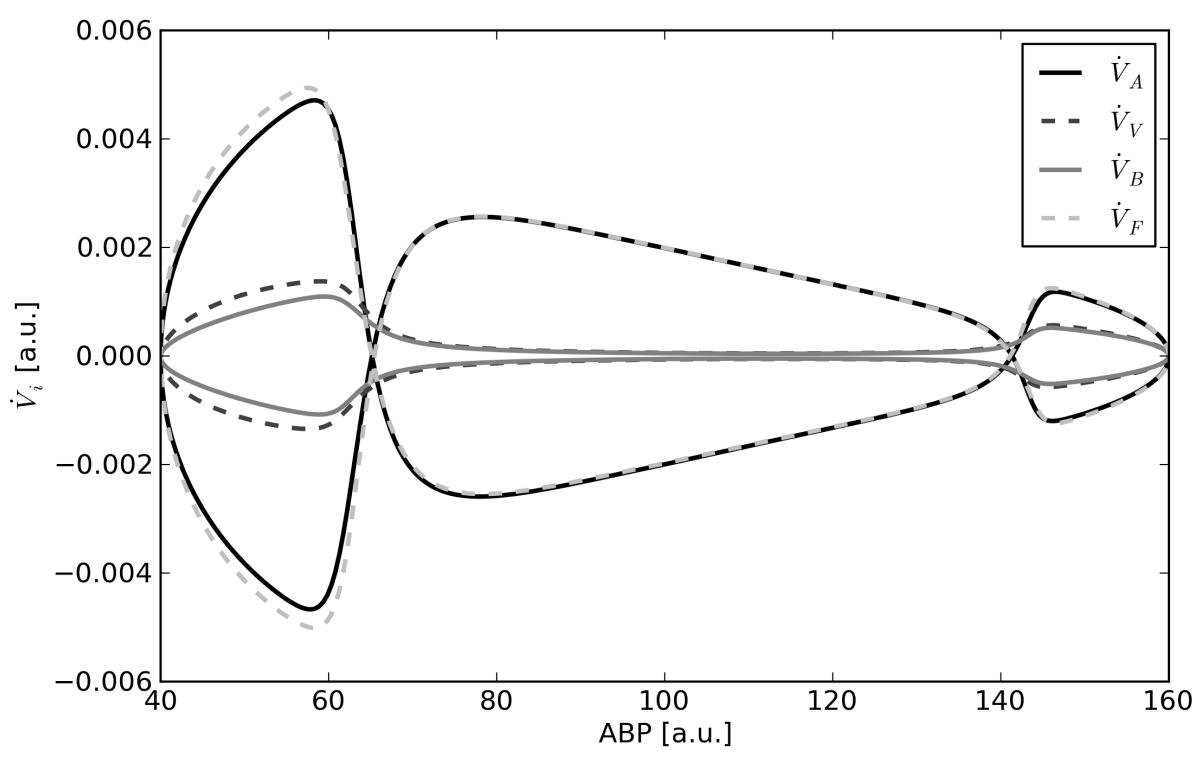

Figure 5.22: $\dot{V}_{i}$ against ABP with $\alpha=2.0$ and $V_{E}=0 \mathrm{ml}$ (bHOM; case 2). 
volume change of the veins and brain is once again anti-correlated, which can be seen in fig. 5.21, as it is for arteries and CSF. From fig. 5.22 it can easily be seen, that for ABP values inside the autoregulation and above, the corresponding changes are nearly identical. For ABP values below, the absolute change of volume for veins is bigger than the volume change of the brain tissue. This difference is absorbed once again by the CSF.

\subsection{Swelling}

The original motivation for developing a model was to better understand the underlying involved processes, and to better interpret measured data from patients with a severe head injury. Normally, with head injuries a swelling of the brain is accompanied.

Therefore, the simulations of the previous section are now repeated, but with the difference of a swelling as big as the standard volume of the fluid compartment $\left(V_{F}=30 \mathrm{ml}\right)$, in order to model a patient with such a severe head injury. This modification should alter the behavior of the simulations in an interesting way, as situations arise, in which there is no CSF left to absorb.

Before the actual simulations can be done, the system has to be prepared for the new state, which should represent the brain with a swelling. A naive approach would be to set the value for $V_{E}=30 \mathrm{ml}$ (the size of the swelling) and therefore subtracting this value from the standard value of the CSF, which would result in $V_{F}=0 \mathrm{ml}$. Here, a different approach was chosen: To prepare the system for this state, in which the swelling reached its maximum, the simulations were started with the standard values and a constant ABP. To simulate the development of the swelling, which does not occur spontaneously, the volume of the swelling $V_{E}$ increases until it reaches its maximum. From this point onwards, the real simulations are done.

\subsubsection{Case 3: swelling, no autoregulation}

As in the previous section, first, the simulations were done with an impaired autoregulation and are compared to the previously obtained results of the corresponding simulations in case 1 with no swelling. This will provide a better insight into the differences between both scenarios.

\section{CBF}

Figure 5.23 depicts side by side the CBF values for the model with and without a swelling.

Both simulations have a similar development for an $\mathrm{ABP}$ of $97 \mathrm{mmHg}$ and below. The difference of both graphs lies in the different gradient of the $\mathrm{CBF}$ for $\mathrm{ABP}$ values above $97 \mathrm{mmHg}$. Here, the $\mathrm{CBF}$ for the simulations 


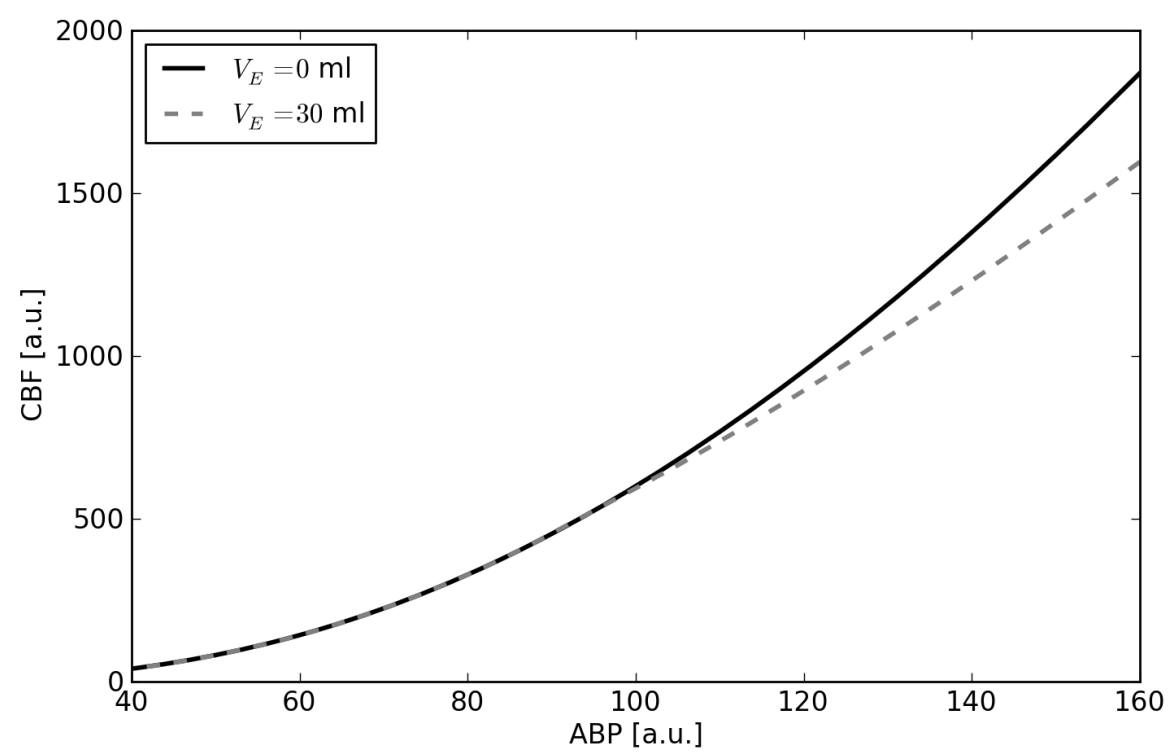

Figure 5.23: $\mathrm{CBF}$ against $\mathrm{ABP}$ for $\alpha=0.0$ and with $\left(V_{E}=30 \mathrm{ml}\right)$ and without $\left(V_{E}=0 \mathrm{ml}\right)$ a swelling (bHOM; case 3 ).

with a swelling has a smaller slope and the maximal CBF value for the highest ABP differs about $250 \mathrm{ml} / \mathrm{min}$.

The reason for this will be deduced in the next sections. But as the difference lies in the swelling and therefore in a diminished CSF volume, it can be assumed, that for increasing ABP values above the default value, there will be no CSF left to absorb.

\section{Pressure}

As before, the run of the pressures $p_{C}, p_{V}$ and $p_{B}$ follows in principal the development of the ABP, which can be deduced from figure 5.24 (see fig 5.2 and 5.3 for comparison), in which the graphs of the pressures inside the compartments are depicted against ABP.

Compared with the scenario without a swelling, the graphs are quite similar, with small differences in the range (compare tables 5.1 and 5.6), except for the ICP: Here, the pressure $p_{B}$ for ABP values below $97 \mathrm{mmHg}$ is similar in both scenarios. But after a sharp bend, it has a stronger increase for values above. As will be seen, this is due to the fact, that for an ABP above $97 \mathrm{mmHg}$, there is no further CSF to absorb, which results in a higher ICP.

Furthermore, comparing the pressures in tables 5.1 and 5.6 directly, the 


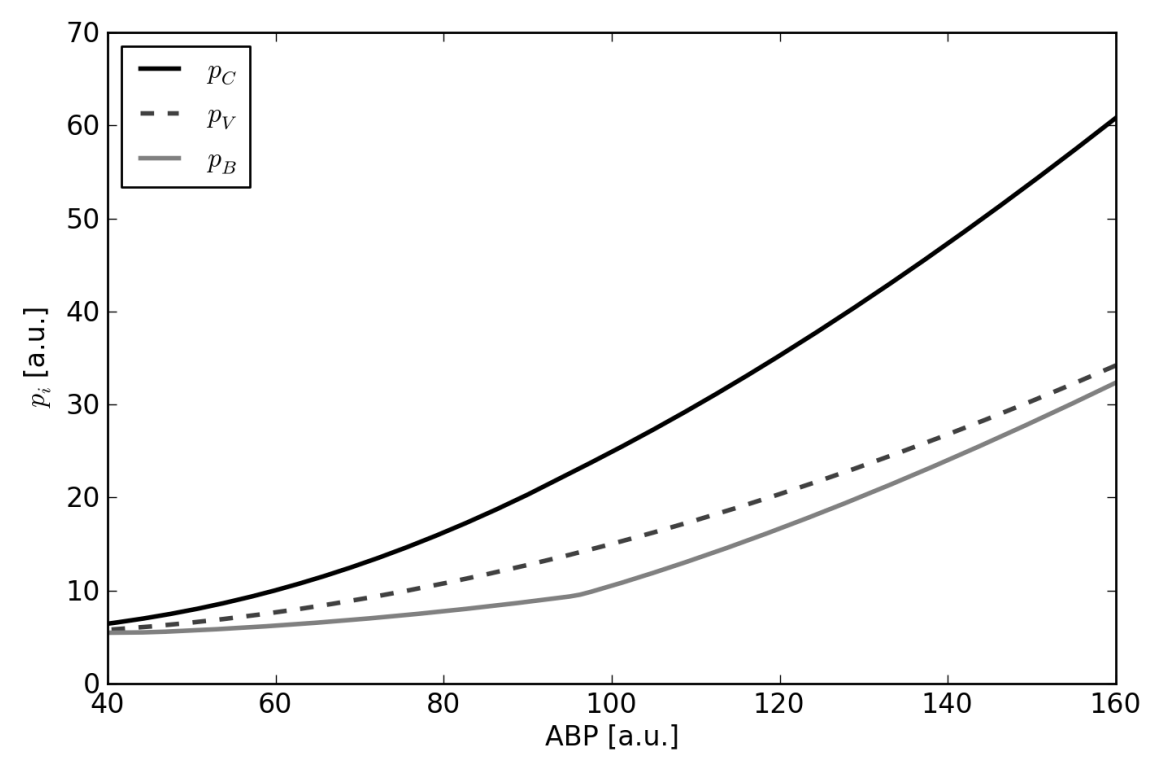

Figure 5.24: $p_{i}$ against ABP with $\alpha=0.0$ and $V_{E}=30 \mathrm{ml}$ (bHOM; case 3).

pressure drop of $3.04 \mathrm{mmHg}$ of the maximum inside the capillaries might be unexpected. But as the resistance in the arteries is increased, due to the decrease of the pressure difference between ABP and ICP (compare eq. 3.6 and next section), the flux into the capillaries decreases and therefore the pressure inside the capillaries.

As for the veins, their volume above $97 \mathrm{mmHg}$ will be smaller, due to the swelling and the resulting overall smaller volume for the other compartments. This does increase the resistance between veins and sagittal sinus. The flux into the sinus is therefore reduced, which will eventually result in a higher pressure inside the veins.

\begin{tabular}{l|r|r|r|r|r} 
Pressure $[\mathrm{mmHg}]$ & $\min$ & default & $\max$ & $\max \left(V_{E}=0\right)$ & range \\
\hline$p_{A}$ & 40.00 & 100.00 & 160.00 & 160.00 & 120.00 \\
$p_{C}$ & 6.44 & 25.00 & 60.81 & 63.85 & 54.37 \\
$p_{V}$ & 5.78 & 15.00 & 34.02 & 32.70 & 28.24 \\
$p_{B}$ & 5.47 & 10.00 & 32.35 & 26.71 & 26.88
\end{tabular}

Table 5.6: Pressure ranges with $\alpha=0.0$ and $V_{E}=30 \mathrm{ml}$ (bHOM; case 3). 


\section{Resistances}

The behavior of the resistances, which was stated in the previous section, can be seen in fig. 5.25.

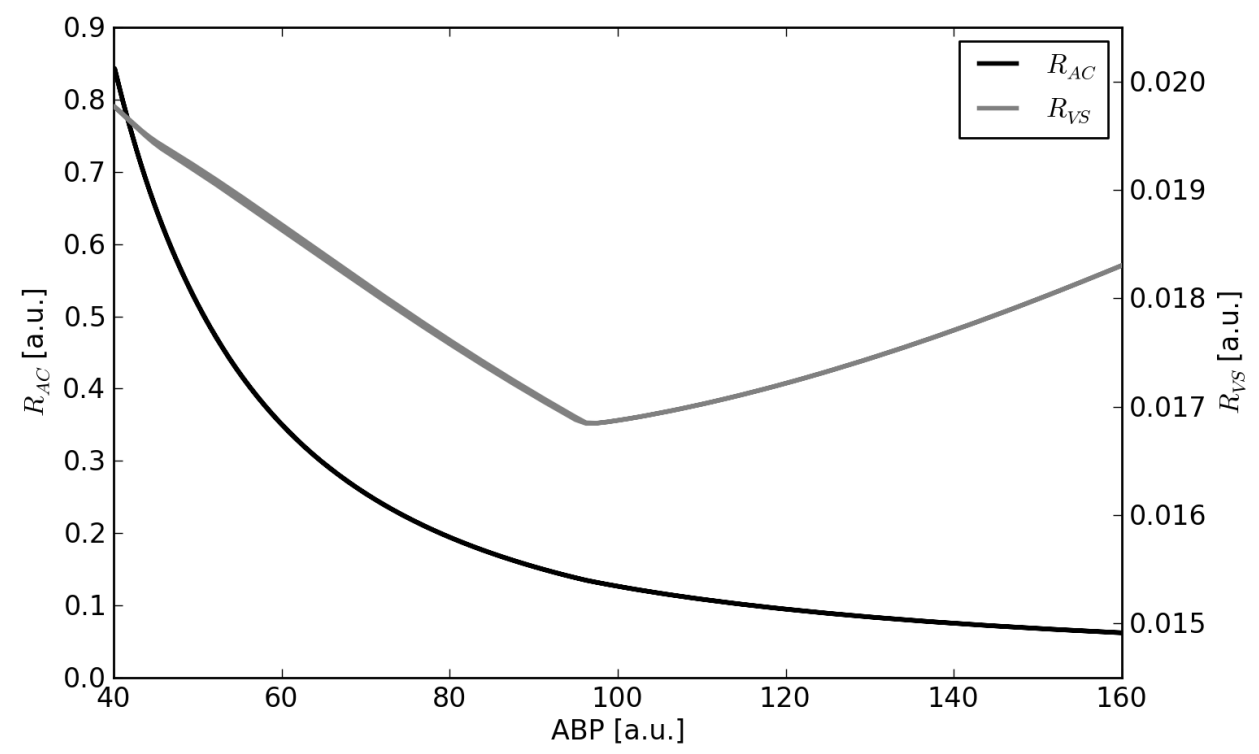

Figure 5.25: $R_{X Y}$ against $\mathrm{ABP}$ with $\alpha=0.0$ and $V_{E}=30 \mathrm{ml}$ (bHOM; case $3)$.

For ABP values below $97 \mathrm{mmHg}, R_{A C}$ is identical to the case with no swelling. For higher ABP values, the resistance inside the arteries is slightly lower. Based on eq. 3.6, $R_{A C}$ depends on the compliance of the arteries and pressure difference between ABP and ICP. As the compliance is constant $(\alpha=0)$, the ABP is equal to and the ICP bigger than in the former scenario, $R_{A C}$ has to be smaller. Table 5.7 summarizes the important values for $R_{A C}$.

\begin{tabular}{l|r|r|r|r} 
Resistance $\left[\frac{\mathrm{mmHg}}{\mathrm{ml} / \mathrm{min}}\right]$ & $\min$ & default & $\max$ & range \\
\hline$R_{A C}\left(V_{E}=30\right) \mathrm{ml}$ & 0.051 & 0.125 & 0.849 & 0.798 \\
$R_{A C}\left(V_{E}=0\right) \mathrm{ml}$ & 0.062 & 0.125 & 0.849 & 0.787
\end{tabular}

Table 5.7: Resistance ranges with $\alpha=0.0$ and $V_{E}=30 \mathrm{ml}$ (bHOM; case 3).

For $R_{V S}$ the increase around $97 \mathrm{mmHg}$ is obvious. Instead of decreasing monotonically, as in the previous case with no swelling, a sharp bend appears at approx. $97 \mathrm{mmHg}$, from where on the resistance increases. As will be seen in a later section, this is due to CSF reservoir depletion. As the total 
volume increase of all other compartments, with increasing ABP, cannot be compensated by the fluid compartment, due to the complete depletion of CSF, the expansion of the veins is restrained by a faster increasing ICP.

\section{Compliances}

The behavior of the compliances, seen in figure 5.26, can easily be explained, with the knowledge gained from the corresponding simulations without a swelling and the pressure curves of the previous section.

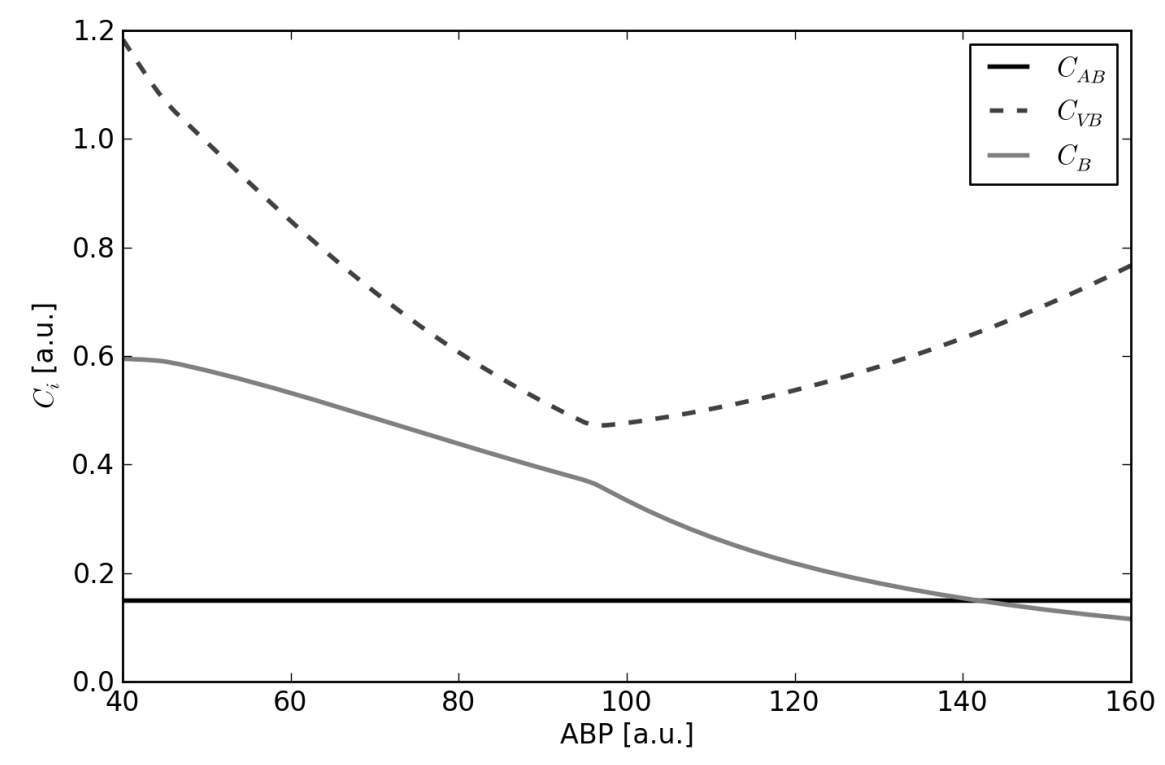

Figure 5.26: $C_{i}$ against $\mathrm{ABP}$ with $\alpha=0.0$ and $V_{E}=30 \mathrm{ml}$ (bHOM; case 3).

As for case 1 , the course of $C_{A B}$ is constant, as there is no active autoregulation.

Due to the same development of the pressure inside each compartment for $\mathrm{ABP}$ values below $97 \mathrm{mmHg}$, the gradients are similar to the former simulations (compare corresponding values from tables 5.2 and 5.8). As for an increasing ABP above approx. $97 \mathrm{mmHg}$, the pressure inside the brain raises more quickly than in case 1 (compare fig. 5.3 and 5.24). Therefore, the compliance of the brain tissue $C_{B}$ decreases faster, as $C_{B}$ is approx. proportional to $1 / p_{B}$.

The compliance of the veins depends on the pressure difference between veins and brain tissue. Therefore, it is approx. proportional to $1 /\left(p_{V}-p_{B}\right)$. As the ICP has a steeper increase than the pressure inside the veins with increasing $\mathrm{ABP}$, with its increase the compliance of the veins has to increase, 


\begin{tabular}{l|r|r|r} 
& \multicolumn{3}{|c}{$\mathrm{ABP}$} \\
Compliance $\left[\frac{\mathrm{ml}}{\mathrm{mmHg}}\right]$ & $40 \mathrm{mmHg}$ & $97 \mathrm{mmHg}$ & $160 \mathrm{mmHg}$ \\
\hline$C_{A B}$ & 0.15 & 0.15 & 0.15 \\
$C_{V B}$ & 1.18 & 0.47 & 0.77 \\
$C_{B}$ & 0.59 & 0.36 & 0.11
\end{tabular}

Table 5.8: Ranges for compliances with $\alpha=0.0$ and $V_{E}=30 \mathrm{ml}$ (bHOM; case 3$)$.

too, contrary to $C_{V B}$ of the former simulations in case 1.

With the previous sections it is now possible to explain the development of the volume of each compartment.

\section{Volumes}

The development of the volumes of each compartment is shown in fig. 5.27. Comparing fig. 5.27 with 5.9, it is easy to see, that for an ABP below $97 \mathrm{mmHg}$, the graphs for each compartment in both scenarios are quite comparable, except for the CSF compartment. Here, $\Delta V_{F}=V_{F}$ and not $V_{F}-V_{F n}$ is plotted, due to the swelling. Above, the graphs begin to differ and for some compartments, they are totally different. The reason for this is the complete depletion of the CSF compartment for an ABP higher than $97 \mathrm{mmHg}$. In this case, the total positive volume change of the other compartments cannot be compensated by the CSF compartment and has to result in a different behavior.

Comparing the deviations of the volume, shown in fig. 5.28 and 5.29, with case 1 (compare fig. 5.10 and 5.11), the difference can be seen more easily: As stated before, for an ABP below $97 \mathrm{mmHg}$, all graphs are identical, even the deviations of the CSF. As long as there is CSF to absorb, the amount of CSF is irrelevant, which can easily be seen from the differential equation system: The system behaves identically for different CSF standard values, as long as $R_{F S}$ is identical. When there is no fluid left to absorb, it becomes infinitely big and the behavior of the system changes.

For the arteries, the explanation for the different behavior above an ABP of $97 \mathrm{mmHg}$ is quite simple: As the ICP raises more quickly, with increasing $\mathrm{ABP}$, due to the lack of CSF left to absorb, the pressure difference of ABP and ICP decreases, as $C_{A B}$ remains constant. Therefore, the absolute change of volume decreases, too. This can easily be seen in fig. 5.29: Here, the right side of the graph for $\dot{V}_{A}$ is quenched compared to fig. 5.11.

For the brain tissue, the absolute deviations are higher than in case 1. As there is no CSF left to absorb, the ICP raises more quickly, due to the higher compression of the brain tissue. Therefore, the compliance of 


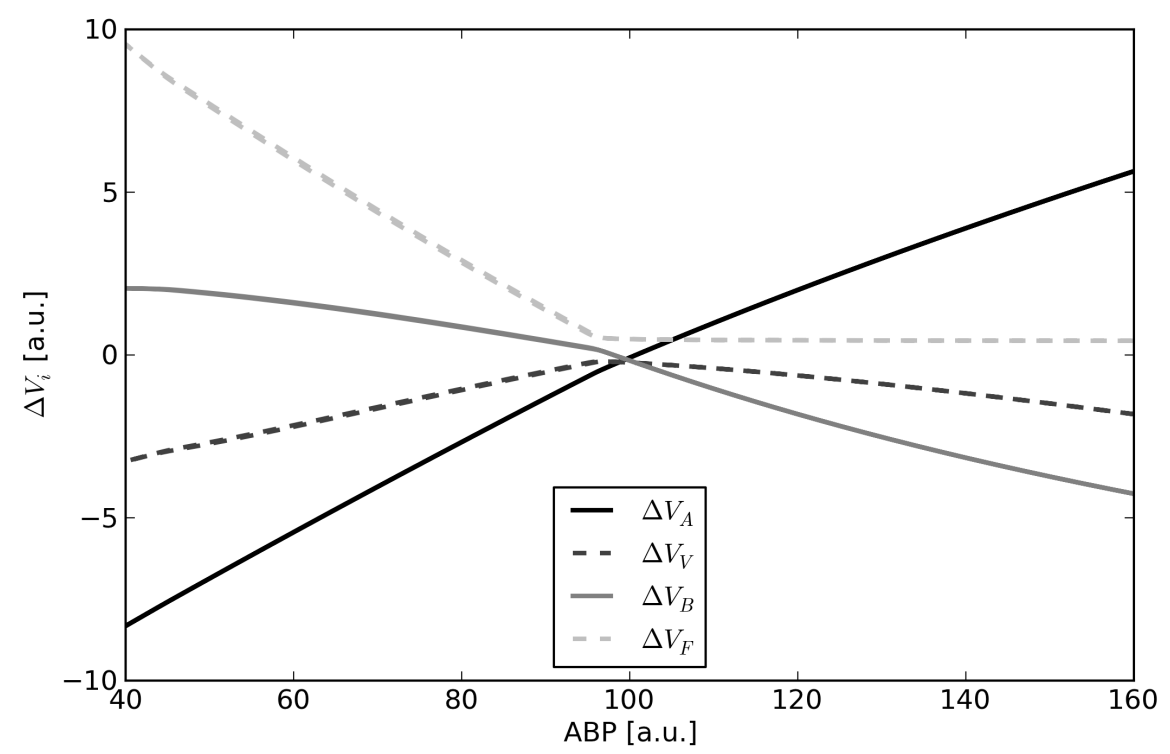

Figure 5.27: $\Delta V_{i}$ against ABP for $\alpha=0.0$ and $V_{E}=30 \mathrm{ml}$ (bHOM; case 3).

the veins increases and with it, the volume of the veins decreases, as the pressure difference between ICP and $p_{V}$ decreases. As the ICP raises more quickly than the pressure inside the veins, the deviation of the volume for the veins is even reverted, when compared to case 1 , in which no swelling occurred.

\subsubsection{Case 4: swelling, autoregulation}

After having examined the behavior of the model for enabled autoregulation and no swelling in section 5.2.2 and disabled autoregulation and a swelling in section 5.3.1, now the simulations are done with enabled autoregulation and a swelling.

\section{CBF}

Taking a look at the CBF, which is depicted in fig. 5.30, the graphs for an enabled autoregulation, but different $V_{E}$, are identical, except for a small segment between an ABP of approx. $50 \mathrm{mmHg}$ and $85 \mathrm{mmHg}$. There, the curve for the simulation, done with a swelling, has slightly smaller values.

The reason for this will become clear, after having a look at the development of the pressures, resistances, compliances and change of volume in the next sections. 


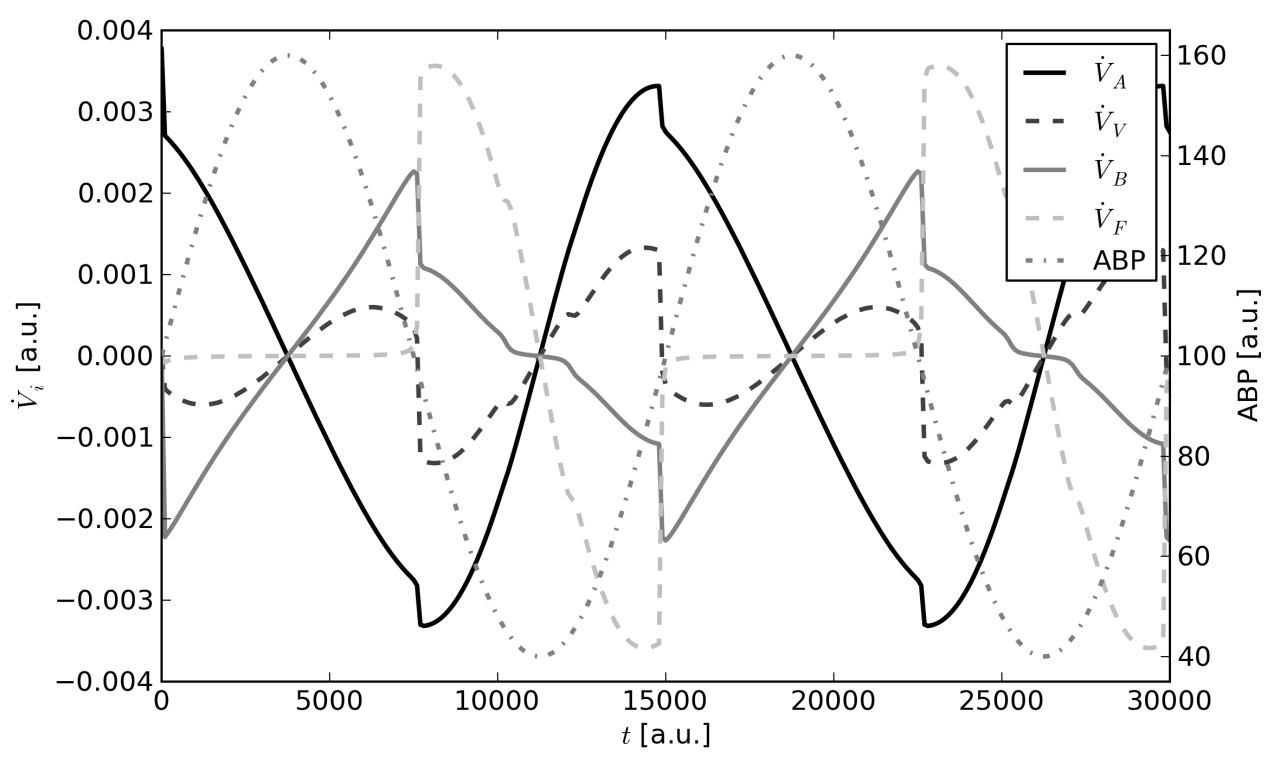

Figure 5.28: $\dot{V}_{i}$ against time for $\alpha=0.0$ and $V_{E}=30 \mathrm{ml}$ (bHOM; case 3).

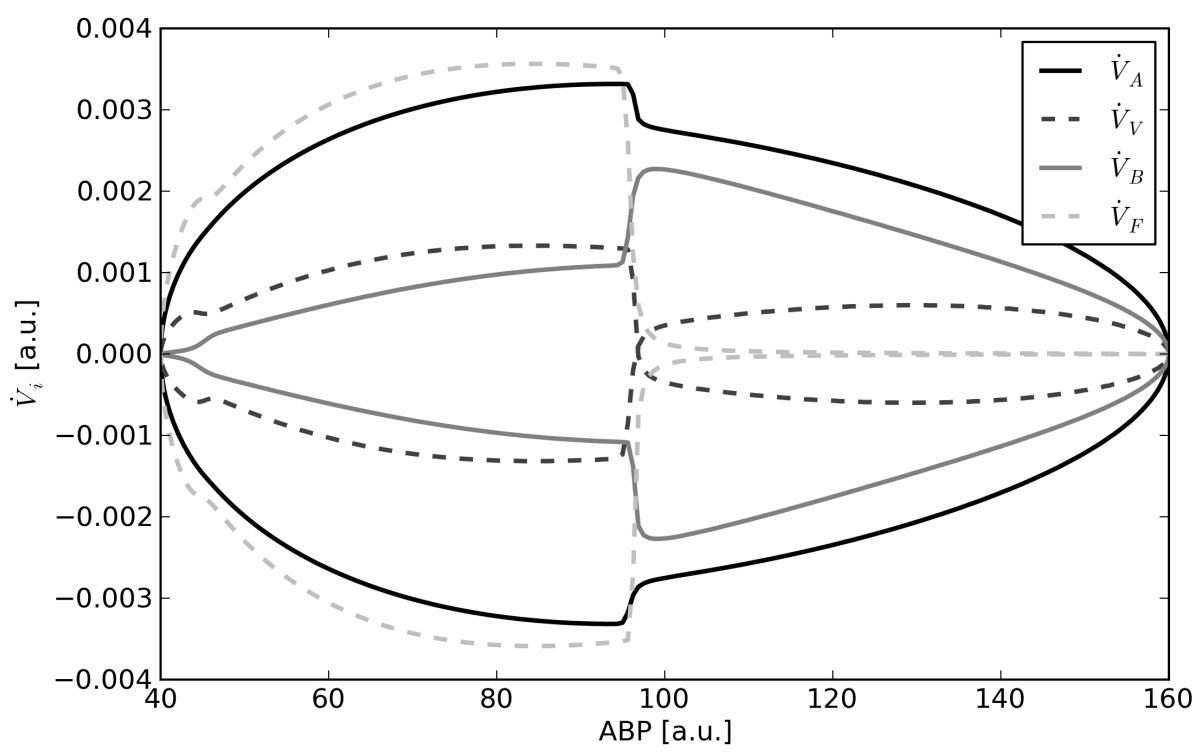

Figure 5.29: $\dot{V}_{i}$ against ABP with $\alpha=0.0$ and $V_{E}=30 \mathrm{ml}$ (bHOM; case 3). 


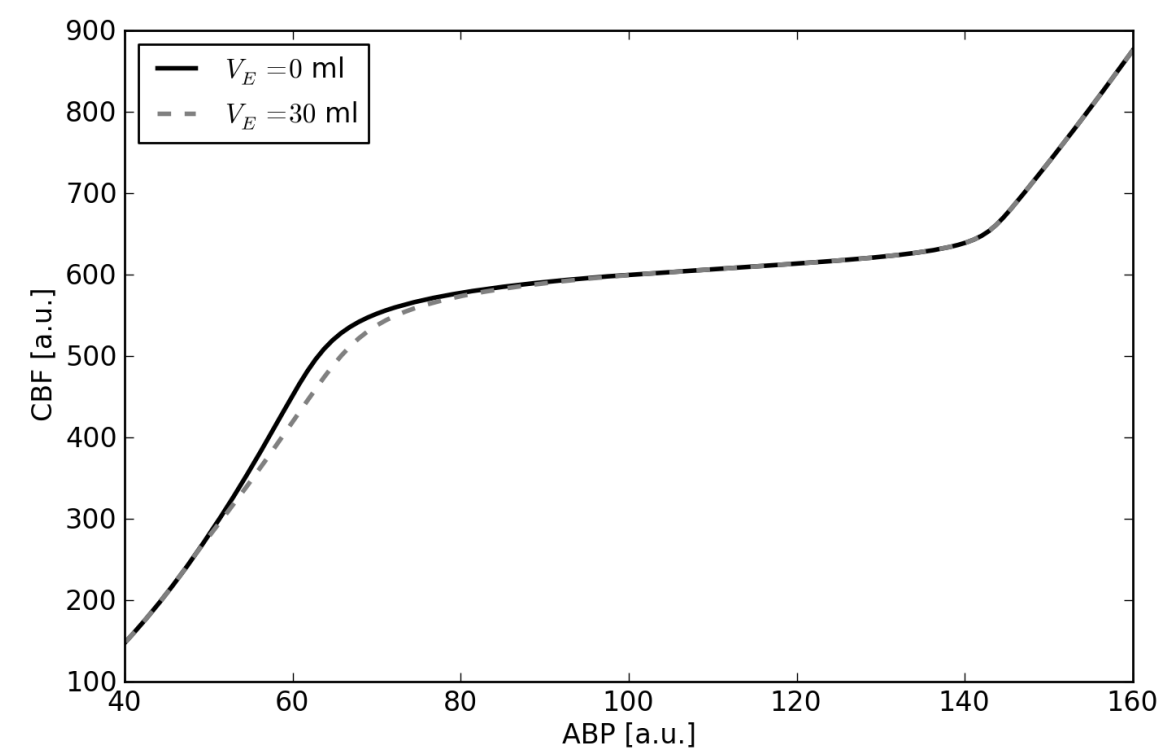

Figure 5.30: $\mathrm{CBF}$ against $\mathrm{ABP}$ for $\alpha=2.0$ and with $\left(V_{E}=30 \mathrm{ml}\right)$ and without $\left(V_{E}=0 \mathrm{ml}\right)$ a swelling (bHOM; case 4$)$.

\section{Pressures}

For the pressures inside each compartment, which is depicted in fig. 5.31, the development of the pressures inside the capillaries and veins is nearly identical to the case without a swelling. But the pressure development of $p_{B}$ differs a lot between an $\mathrm{ABP}$ of approx. $50 \mathrm{mmHg}$ and $105 \mathrm{mmHg}$ from the corresponding scenario.

From the previous sections it can be deduced, that between this interval, due to the swelling, there is no CSF left to absorb, which results in a stronger increase of ICP between this interval, as the volume of the arteries is increased, due to its dilation. The development of the CSF volume will be shown in a following section.

Taking a closer look at the pressure development of the other compartments, this increase can be seen, too. But the effect is much smaller, as it is smoothed out, due to the autoregulation.

\section{Resistances}

The graphs for the resistances $R_{A C}$ and $R_{V S}$ are shown in figure 5.32. As for the pressure $p_{C}$, the graph for $R_{A C}$ looks similar, on a first glance, to the corresponding simulation without a swelling. Taking a closer look (compare with fig. 5.18), one recognizes the slightly increased $R_{A C}$ between an $\mathrm{ABP}$ 


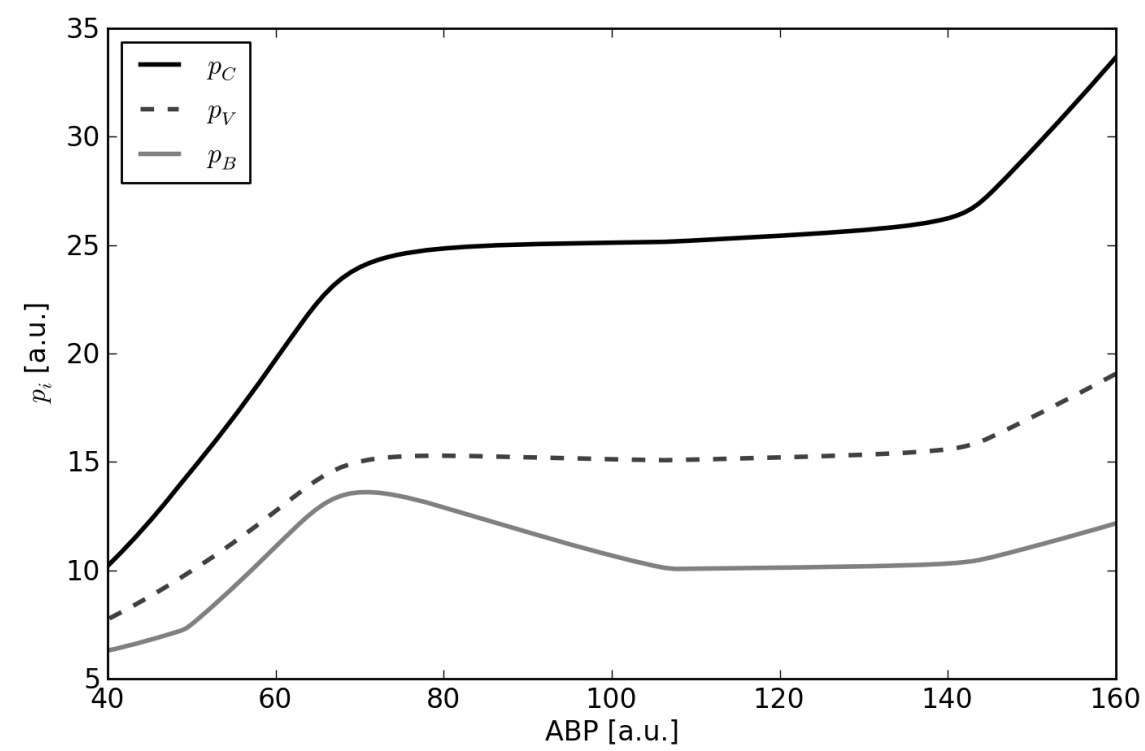

Figure 5.31: $p_{i}$ against $\mathrm{ABP}$ with $\alpha=2.0$ and $V_{E}=30 \mathrm{ml}$ (bHOM; case 4).

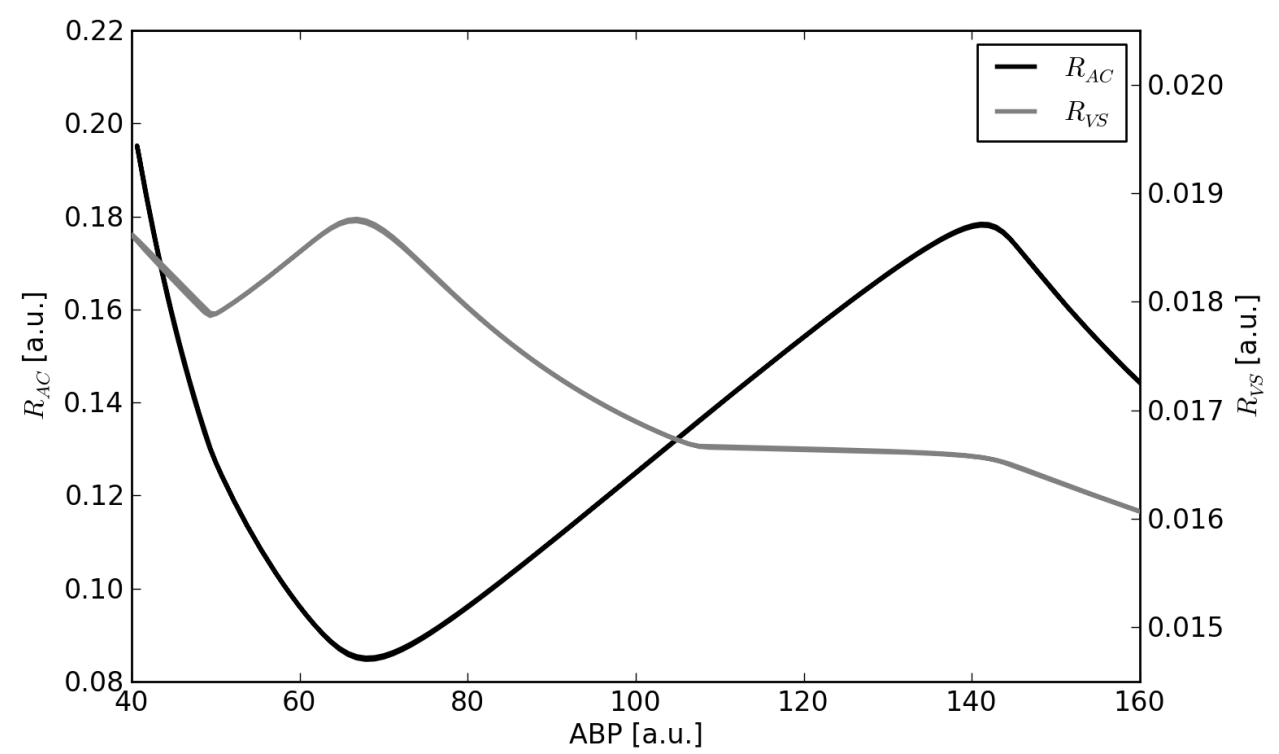

Figure 5.32: $R_{X Y}$ against ABP with $\alpha=2.0$ and $V_{E}=30 \mathrm{ml}$ (bHOM; case 4). 
range of about 50 to $105 \mathrm{mmHg}$. This increase is responsible for the CBF drop in the same interval, shown in the previous section about CBF. As before, the cause of the increased value lies in the increased ICP.

As for $R_{V S}$, the bump, seen in the ICP, can also be seen in the corresponding resistance. The reason for this is simple: As the brain tissue imposes more pressure upon the veins, their volume decreases more, when compared to case 2, and therefore the resistance of the veins is increased. Outside, both the resistance of the arteries and the resistance of the veins behave as before.

\section{Compliances}

Due to the different development of the pressures, the corresponding compliances will change, too. Figure 5.33 shows the associated graphs.

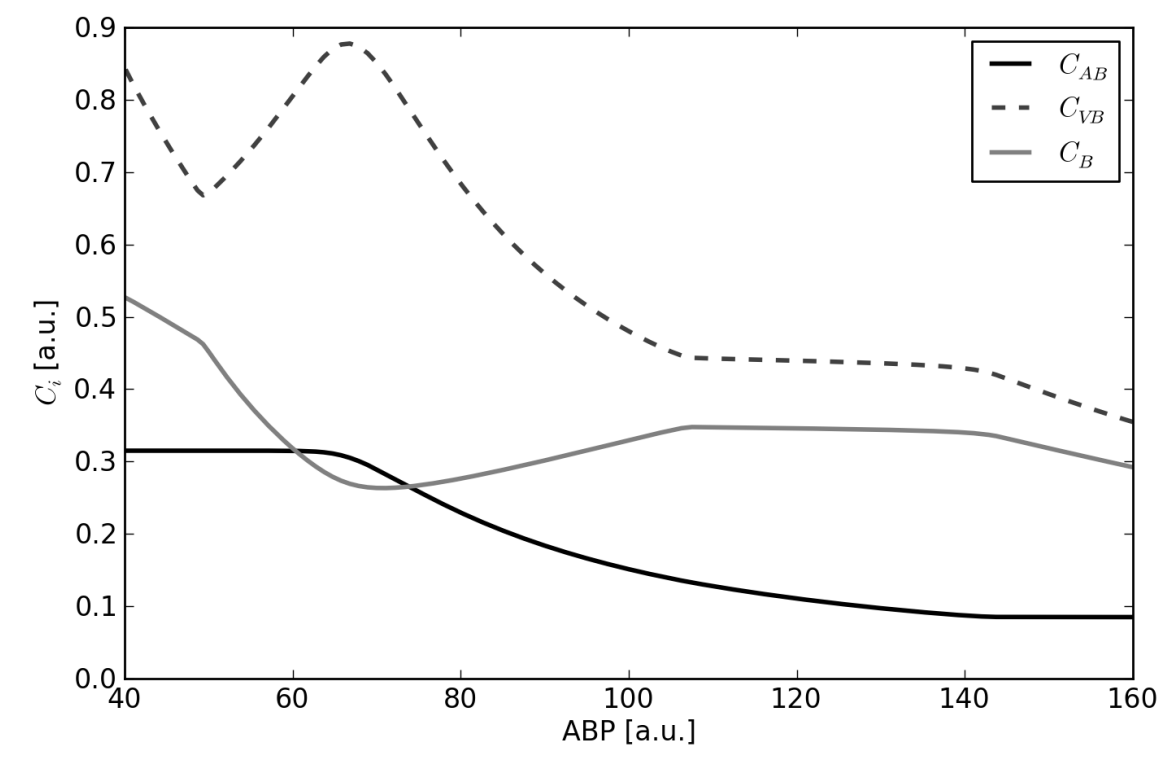

Figure 5.33: $C_{i}$ against ABP with $\alpha=2.0$ and $V_{E}=30 \mathrm{ml}$ (bHOM; case 4).

The shape of the compliance of the arteries has an identical maximum and minimum value for the highest and smallest $\mathrm{ABP}$, as in case 2. The difference between the case without a swelling lies in the fact, that here, the compliance reaches its maximum value for a higher $\mathrm{ABP}$ value, at around $67 \mathrm{mmHg}$, than before. As the veins are more compressed, due to the increased ICP, for decreasing ABP below $100 \mathrm{mmHg}$ the autoregulation compensates this by a bigger increase of their compliance, in order to increase their volume. Therefore, they reach their maximum value earlier. From this 
point on, the CBF can no longer be stabilized and has to drop more quickly with decreasing ABP, which is what can be seen in fig. 5.30.

The reason for the difference of the development of $C_{B}$ and $C_{V B}$ can easily be explained: With the increase of the ICP, the compliance of the brain tissue has to decrease in the corresponding interval, compared to case 2 (compare eq. 3.18). On the contrary, as $C_{V B}$ depends on the pressure difference $p_{V}-p_{B}$, according to eq. 3.12 , the bigger increase in $p_{B}$ results in an increase of $C_{V B}$, which is exactly what can be seen in fig. 5.33 .

As before, outside the $\mathrm{ABP}$ range of about $50 \mathrm{mmHg}$ to $105 \mathrm{mmHg}$, the values for the compliances are identical to the no swelling case.

\section{Volumes}

Looking at fig. 5.34, the graph of $\Delta V_{F}$ (as in case 3 , here, $\Delta V_{F}=V_{F}$ ) verifies the previously made proposition, that between 50 and $105 \mathrm{mmHg}$, there is no CSF left to absorb. As before, this is the only reason, why the simulations with and without a swelling differ.

The differences and similarities between both simulations are much more obvious, by looking at the deviations of the volumes, which are depicted against ABP in fig. 5.35. As stated before, for intervals of ABP, where there is still CSF left to compensate the volume changes of the other compartments, both simulations behave similarly. But inside the interval, in which the CSF compartment is completely depleted, the deviations differ.

As there is no CSF left to absorb, the brain tissue is more compressed, which results in a bigger compression of the veins than before, due to the increased ICP.

The volume change of the arteries happens in a different interval. As long as the active part of the arteries has not reached its maximum, which is around $65 \mathrm{mmHg}$, the graphs for different $V_{E}$ are similar. But as this maximum is reached, the deviation of $V_{A}$ changes. As the compliance of the arteries can no longer be altered, the volume change will be smaller with reduced $\mathrm{ABP}$, compared to case 2, as can be seen in fig. 5.34 compared to fig. 5.20.

\subsection{Summary}

In this chapter, the behavior of the bHOM model was examined. Therefore, simulations for four scenarios were analyzed. In the first part, the behavior of the model was examined without a swelling. In the first case the autoregulation mechanism was disabled, as this marks the simplest state, from a model point of view. Afterwards the simulations were repeated with an enabled autoregulation, as this scenario represents the system under normal circumstances. It was shown, how the compartments interact and influence 


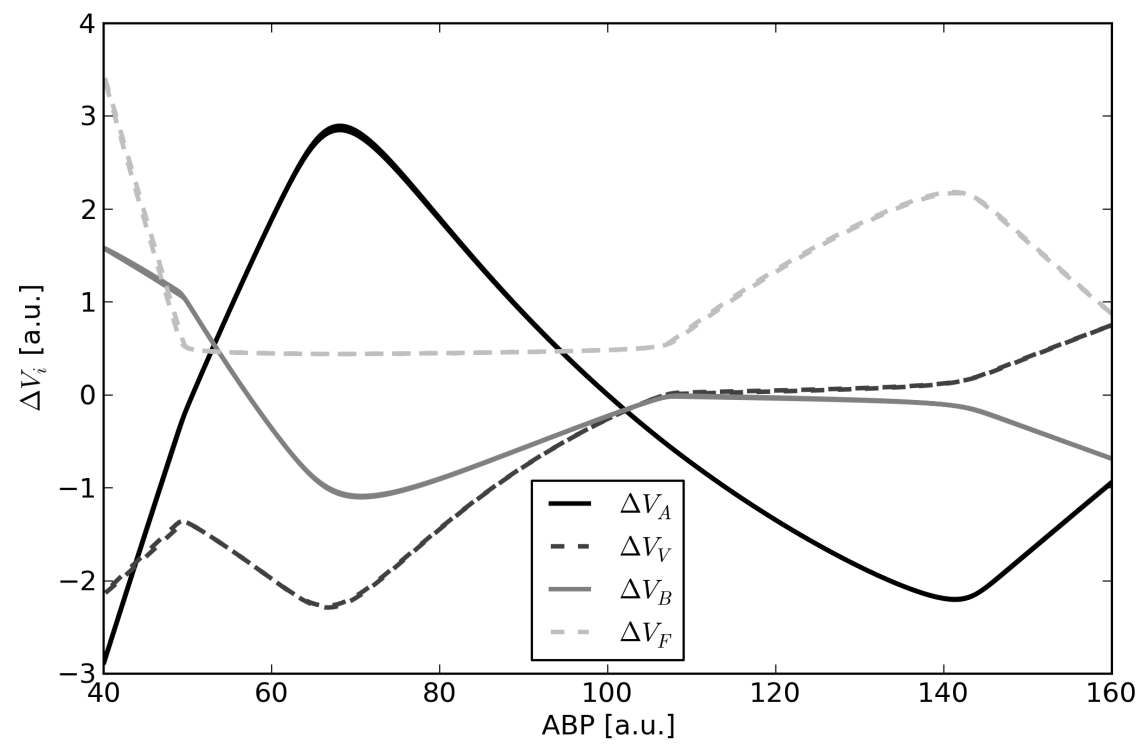

Figure 5.34: $\Delta V_{i}$ against $\mathrm{ABP}$ with $\alpha=2.0$ and $V_{E}=30 \mathrm{ml}$ (bHOM; case $4)$.

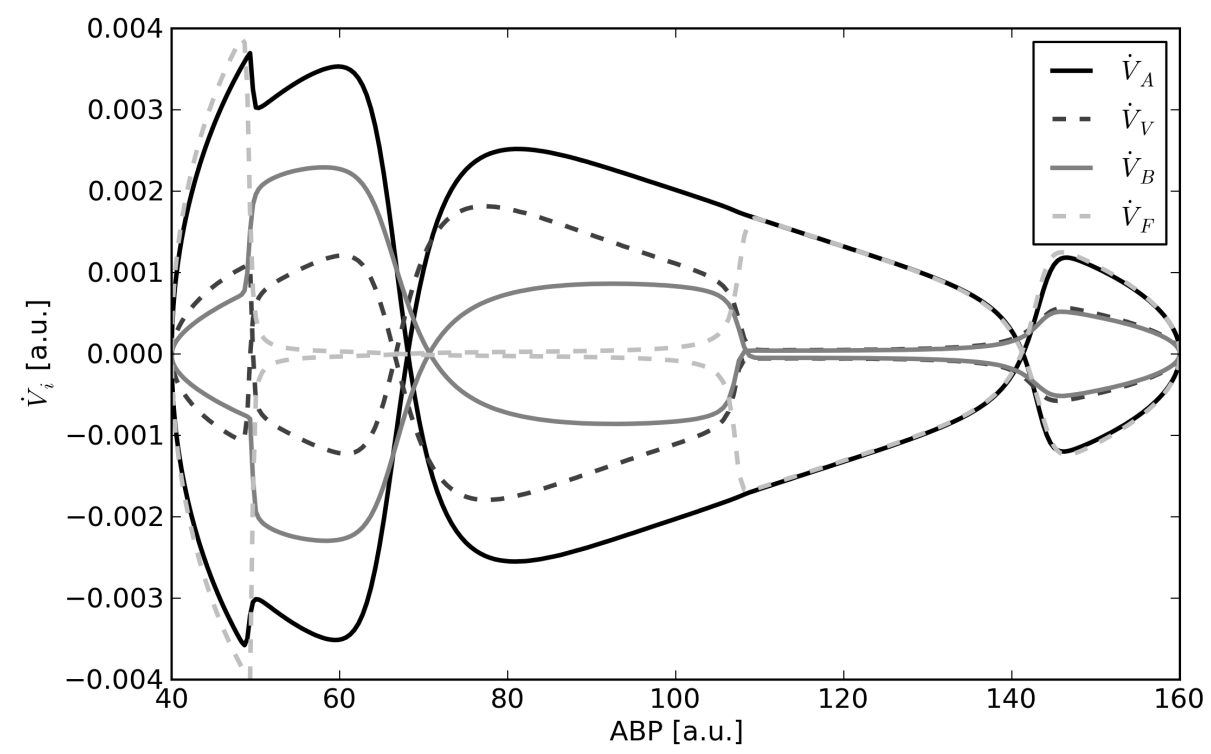

Figure 5.35: $\dot{V}_{i}$ against ABP with $\alpha=2.0$ and $V_{E}=30 \mathrm{ml}$ (bHOM; case 4). 
each other, especially how the autoregulation mechanism alters the behavior of the system.

In the second part, both simulations were repeated with the difference, that a swelling was assumed. It was shown, that in the simulations there is no real difference between the simulations with and without a swelling, as long as there is CSF left to absorb. When the CSF compartment is depleted, the system can behave in a quite different way, due to the increase in ICP.

But the bHOM model has also shown its weaknesses: As mentioned in a previous section, the vein compartment behaves in a strange way. Also, the influence of the ICP on the CBF is smaller than expected, which suggests, that the effect of the ICP on the vein compartment has to be altered. Therefore, in the next chapter the vein compartment will be altered by using the so called starling resistor. 


\section{Chapter 6}

\section{General behavior of the sHOM model}

In the previous chapter, the general behavior of the basic model (bHOM) was examined for four different cases. As mentioned before, there are small inconsistencies in the basic model: For one, the volume development of the veins has an odd behavior for disabled autoregulation for an arterial blood pressure (ABP) around $60 \mathrm{mmHg}$. Additionally, the differences between the simulations with and without a swelling are much less than expected.

In this chapter, the simulations of the previous chapter are repeated, but here, the vein compartment will be modeled differently, to overcome these inconsistencies. Therefore, the resistance of the veins will be modeled as a starling resistor, as described in section 3.4.1. Furthermore, this approach has the advantage, that the venous system can collapse, which is not possible in the previous approach.

To simplify matters, mainly the differences between the basic and the starling model (sHOM) will be described and therefore, the structure of the previous chapter is reused.

\subsection{No swelling}

Similar to the previous chapter, at first, the simulations for the sHOM model are done with dis- $(\alpha=0)$ and enabled $(\alpha=2)$ autoregulation mechanism and no swelling $\left(V_{E}=0 \mathrm{ml}\right)$ inside the brain.

\subsubsection{Case 1: no swelling, no autoregulation}

In figure 6.1, the cerebral blood flow (CBF) of the bHOM and sHOM model are shown for a direct comparison. The simulations were done for disabled autoregulation and no swelling. 


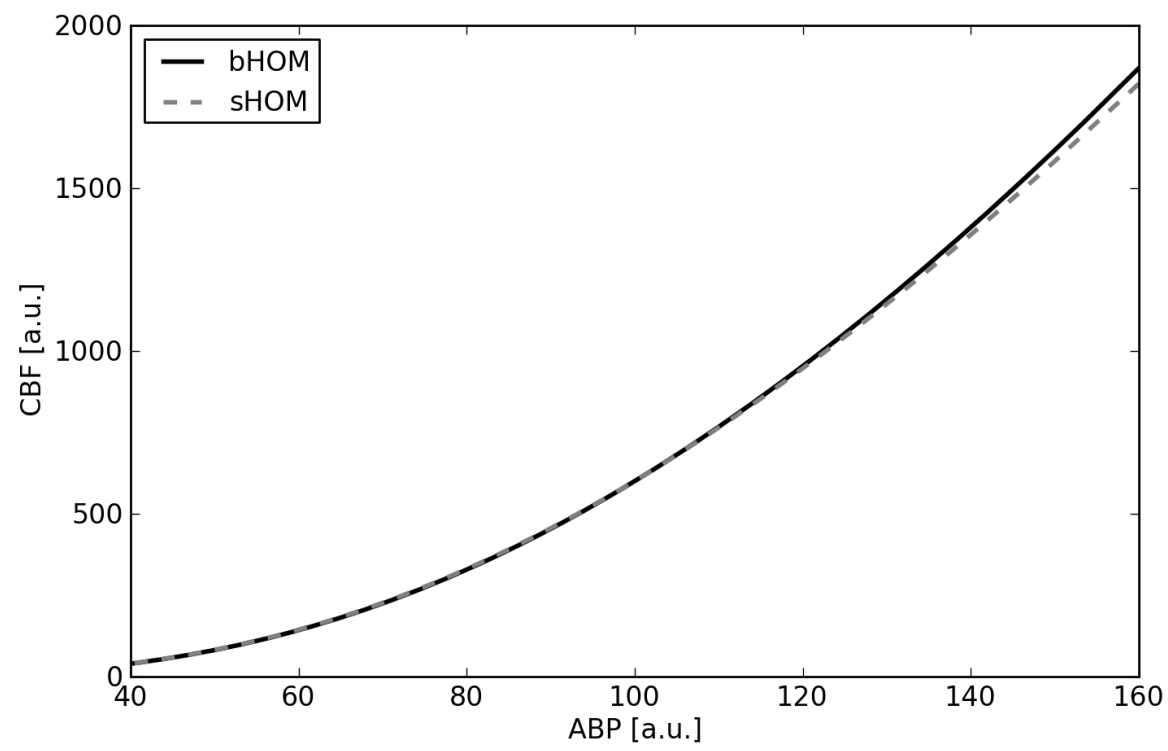

Figure 6.1: $\mathrm{CBF}$ against $\mathrm{ABP}$ with $\alpha=0.0$ and $V_{E}=0 \mathrm{ml}$ (bHOM and sHOM; case 1).

As it can be seen, inside the used ABP range between 40 to $160 \mathrm{mmHg}$, the curves differ only slightly. For ABP values below around $110 \mathrm{mmHg}$, both curves have more or less the same slope, whereas for higher ABP values, the $\mathrm{CBF}$ for the sHOM model stays below the bHOM model with increasing gap.

The cause for the difference between both curves will be examined in the following sections.

\section{Pressure}

The curves for the pressure inside the capillaries, veins and brain tissue are depicted in figure 6.2. The pressure curves have a similar slope as those of the bHOM model (compare fig. 5.3), which is the expected behavior and may be assumed from the CBF curves.

The ranges differ slightly, which can be seen in table 5.1 (bHOM) and 6.1 ( $\mathrm{sHOM}$ ). The minimal values for the pressures are nearly identical, whereas the pressure inside the veins has the biggest deviation and is about $0.02 \mathrm{mmHg}$ higher in case of the sHOM model. As for the maximum pressure inside each compartment, the values for the sHOM case are approximately $2 \mathrm{mmHg}$ higher (compare tables for the exact difference), but are still in the same order of magnitude. 


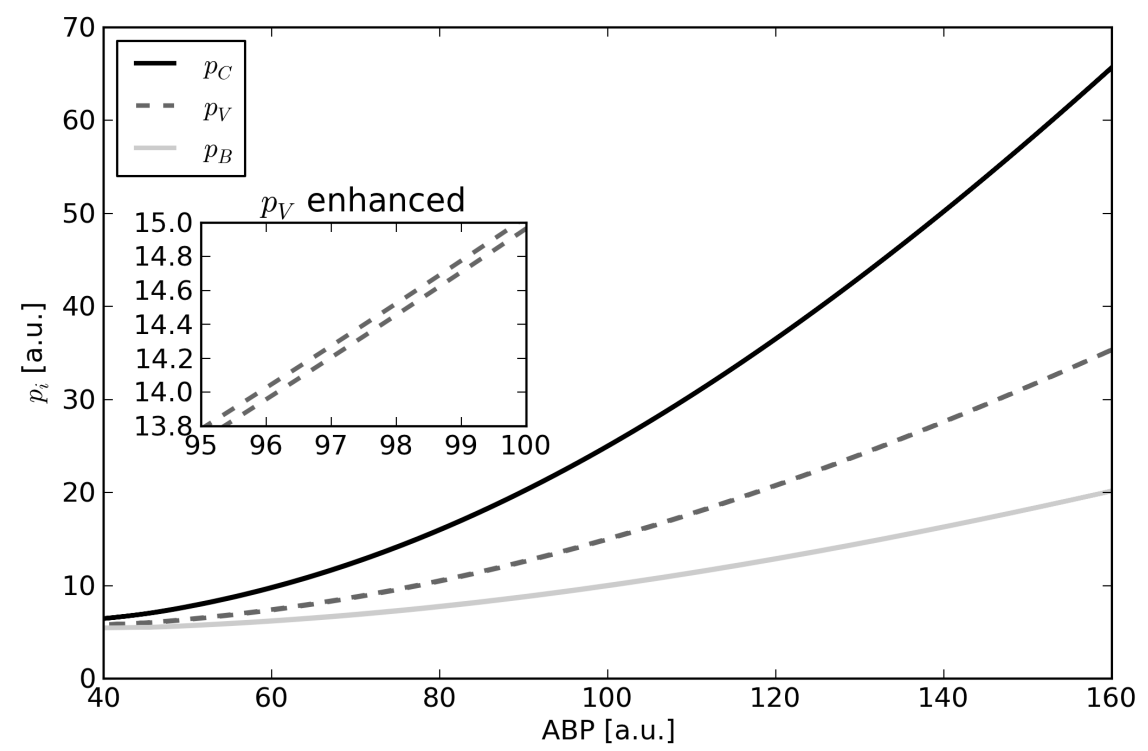

Figure 6.2: $p_{i}$ against ABP with $\alpha=0.0$ and $V_{E}=0 \mathrm{ml}$ (sHOM). A magnified section for $p_{V}$ between an $\mathrm{ABP}$ of $95 \mathrm{mmHg}$ and $100 \mathrm{mmHg}$ is show, to visualize the splitting of $p_{V}$.

In addition to the graphs of the pressures, an magnified plot for $p_{V}$ between an ABP of $95 \mathrm{mmHg}$ and $100 \mathrm{mmHg}$ is shown. As can be seen, there occurs a splitting in this graph. Responsible for the splitting may be numerical inaccuracies of the Runge Kutta Solver and will have an enhanced effect on other simulated values, as will be seen in the following sections. $p_{V}$ was shown as an example, but $p_{C}$ and $p_{B}$ are also affected.

\begin{tabular}{l|r|r|r|r} 
Pressure $[\mathrm{mmHg}]$ & min & default & $\max$ & range \\
\hline$p_{A}$ & 40.00 & 100.00 & 160.00 & 120.00 \\
$p_{C}$ & 6.45 & 25.00 & 65.70 & 59.25 \\
$p_{V}$ & 5.80 & 15.00 & 35.35 & 29.55 \\
$p_{B}$ & 5.47 & 10.00 & 20.17 & 14.70
\end{tabular}

Table 6.1: Pressure ranges with $\alpha=0.0$ and $V_{E}=0 \mathrm{ml}$ (sHOM; case 1).

\section{Resistances}

The development of the resistance of the veins for the bHOM and sHOM model are shown in figure 6.3 and 6.4. As the graph of the resistance of the arteries is nearly identical, it is left out. 


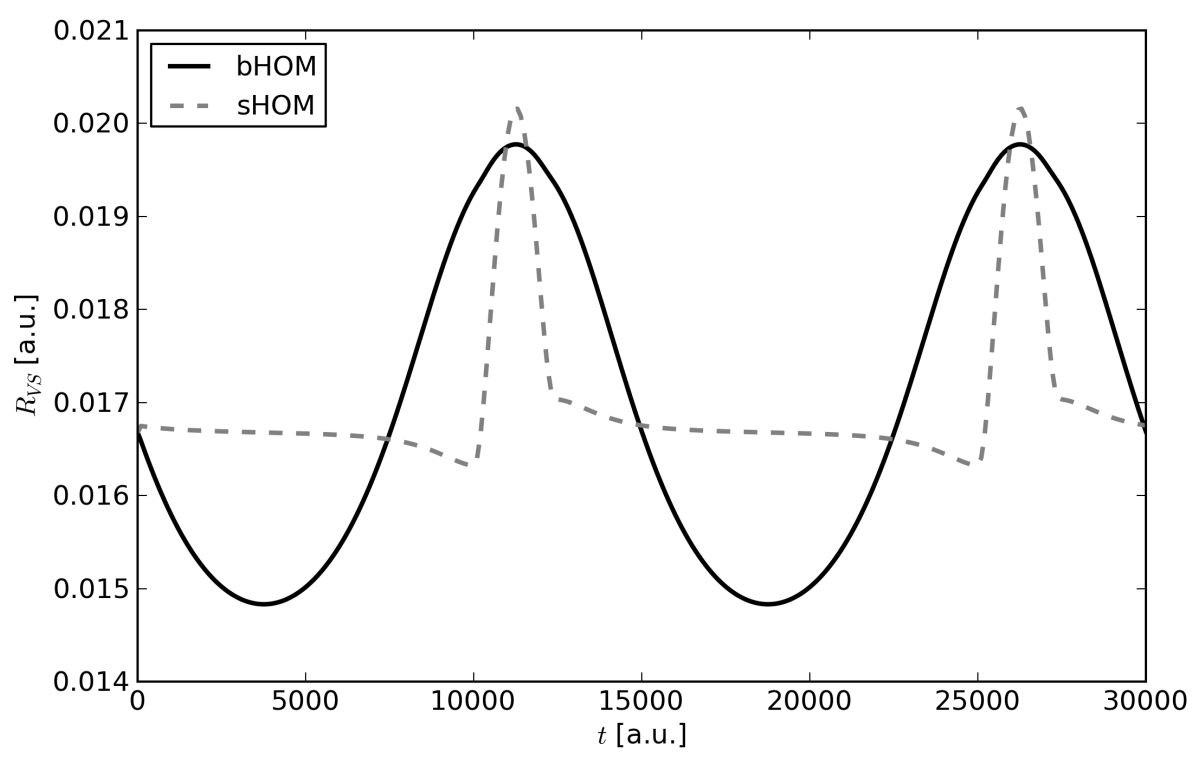

Figure 6.3: $R_{V S}$ against time with $\alpha=0.0$ and $V_{E}=0 \mathrm{ml}$ (bHOM and sHOM; case 1).

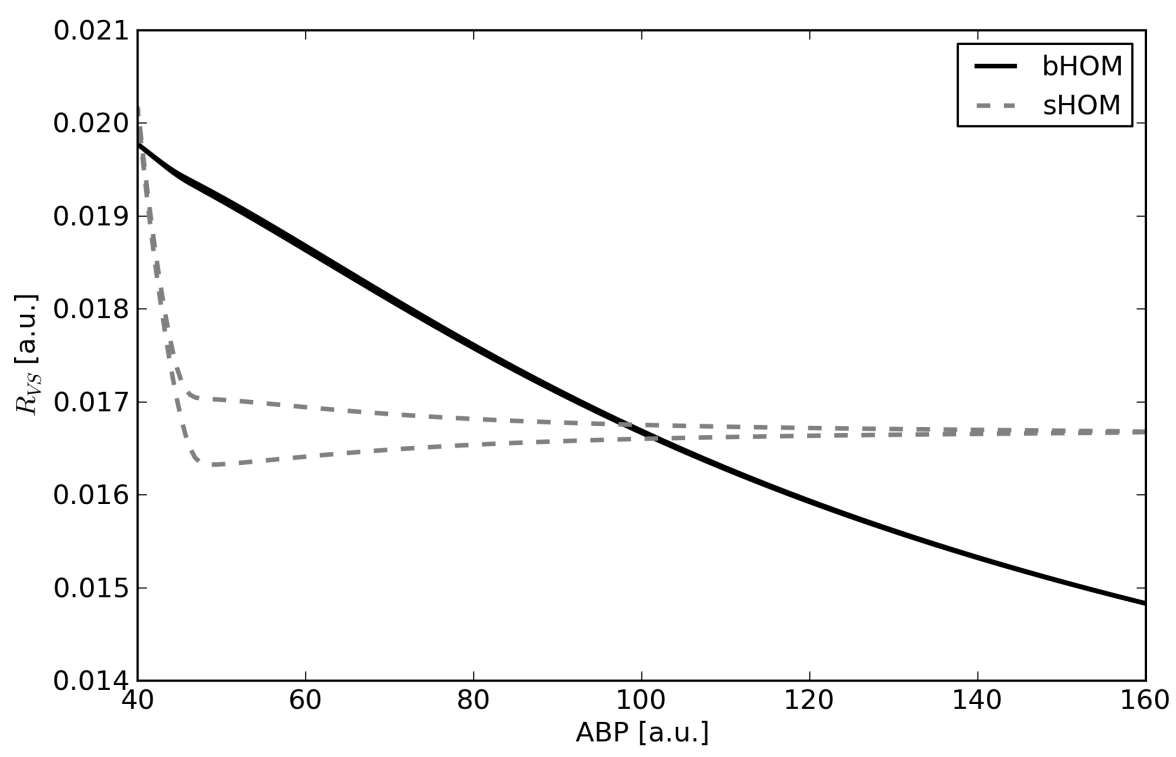

Figure 6.4: $R_{V S}$ against $\mathrm{ABP}$ with $\alpha=0.0$ and $V_{E}=0 \mathrm{ml}$ (bHOM and sHOM; case 1). 
The slope of $R_{V S}$ is totally different for both models. In the bHOM case, the development follows more or less a line with a descending slope. But for the sHOM case, the graph, as seen in fig. 6.4, has a steep descending slope in the beginning, and a sharp bend around $48 \mathrm{mmHg}$, from where on it converges around $0.01667 \frac{\mathrm{mmHg}}{\mathrm{ml} / \mathrm{min}}$.

To explain the behavior, equation 3.45 in its simpler form can be used:

$$
R_{V S}=g_{V S} \frac{p_{V}-p_{S}}{p_{V}-p_{B}}
$$

The splitting of the resistance, which can quite clearly be seen in figure 6.4, stems from the splitting of the pressure inside the veins (compare fig. 6.2). Interestingly, the splitting of the vein pressure and intracranial pressure (ICP), which was not magnified in fig. 6.2, cancel each other out in the difference between both.

The more interesting behavior is the convergence around $0.01667 \frac{\mathrm{mmHg}}{\mathrm{ml} / \mathrm{min}}$ for increasing ABP values, as this is the value of $g_{V S}$ times two.

From this it follows, that the deviation of $p_{V}$ for increasing ABP converges to the deviation of ICP times two, which means, that the normalized* pressure inside the veins increases twice as fast as the normalized ICP. The splitting of $R_{V S}$ has a maximum variation of about $2.5 \%$ and therefore, it can be neglected in further discussions.

Below an ABP of $47 \mathrm{mmHg}$ this development changes, whereas the pressure inside the veins converges more slowly to $5 \mathrm{mmHg}$ than ICP, which results in the raise of $R_{V S}$ for decreasing $\mathrm{ABP}$ values.

\section{Compliances}

The development of the compliances can be considered equal to the bHOM model. There are slight differences in the minimal and maximal values, but the range has approximately the same magnitude, as it was for the corresponding pressure values. Table 6.2 gives the minimal and maximal values, which can be compared with table 5.2.

\begin{tabular}{l|r|r|r} 
Compliance $\left[\frac{\mathrm{ml}}{\mathrm{mmHg}}\right]$ & $\min$ & $\max$ & range \\
\hline$C_{A B}$ & 0.15 & 0.15 & 0.00 \\
$C_{V B}$ & 0.19 & 1.18 & 0.99 \\
$C_{B}$ & 0.18 & 0.59 & 0.41
\end{tabular}

Table 6.2: Ranges for compliances with $\alpha=0.0$ and $V_{E}=0 \mathrm{ml}$ (sHOM; case 1$)$.

\footnotetext{
${ }^{*}$ Here, normalized means value of pressure minus its possible minimum value, which is equal to $p_{S}=5 \mathrm{mmHg}$.
} 


\section{Volumes}

The development of the volumes is shown in fig. 6.5 (for $\Delta V_{i}$ ) and fig. 6.6 (for $\dot{V}_{i}$ ). As it could have been concluded from the previous sections, the graphs show only small deviations from the corresponding graphs of the bHOM model.

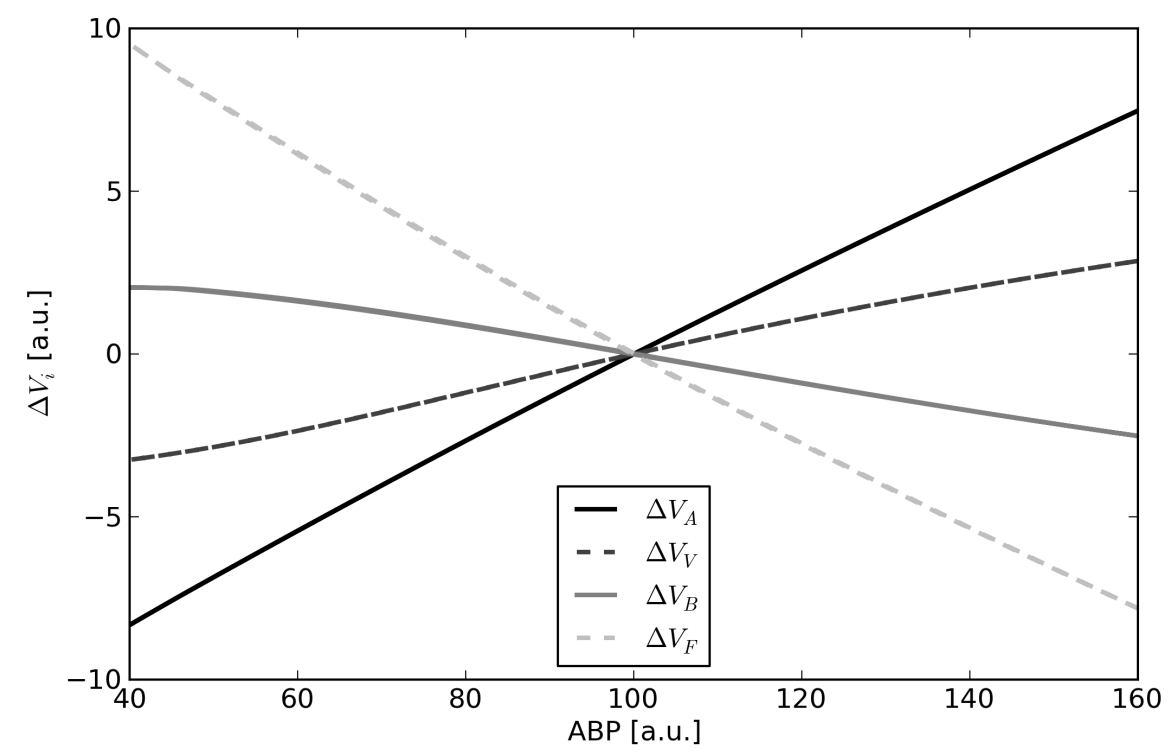

Figure 6.5: $\Delta V_{i}$ against $\mathrm{ABP}$ with $\alpha=0.0$ and $V_{E}=0 \mathrm{ml}$ (sHOM; case 1).

One might ask the question, why the splitting of $R_{V S}$, seen in fig. 6.4, does not have any impact on the dependent graphs. But $R_{V S}$ is only needed to calculate the deviation of the volume of the veins:

$$
\dot{V}_{V}=\frac{p_{C}-p_{V}}{R_{C V}}+\frac{p_{S}-p_{V}}{R_{V S}}
$$

As the splitting stems from the pressure difference between the veins and the sinus, the effect disappears, as the corresponding term can be canceled from the fraction (compare eq. 6.1) and therefore plays no role in the equation.

Another difference between both models is the smoother development of the volumes (compare fig. 5.11 and 6.6). The strange behavior of the bHOM model at an ABP around $46 \mathrm{mmHg}$, where the development of the volume of the veins was not monotonic, is gone.

For all other measurements, the change of volume of each compartment is in the same order of magnitude as in the previous simulation. The development of the volume of the arteries is nearly identical, which can be 


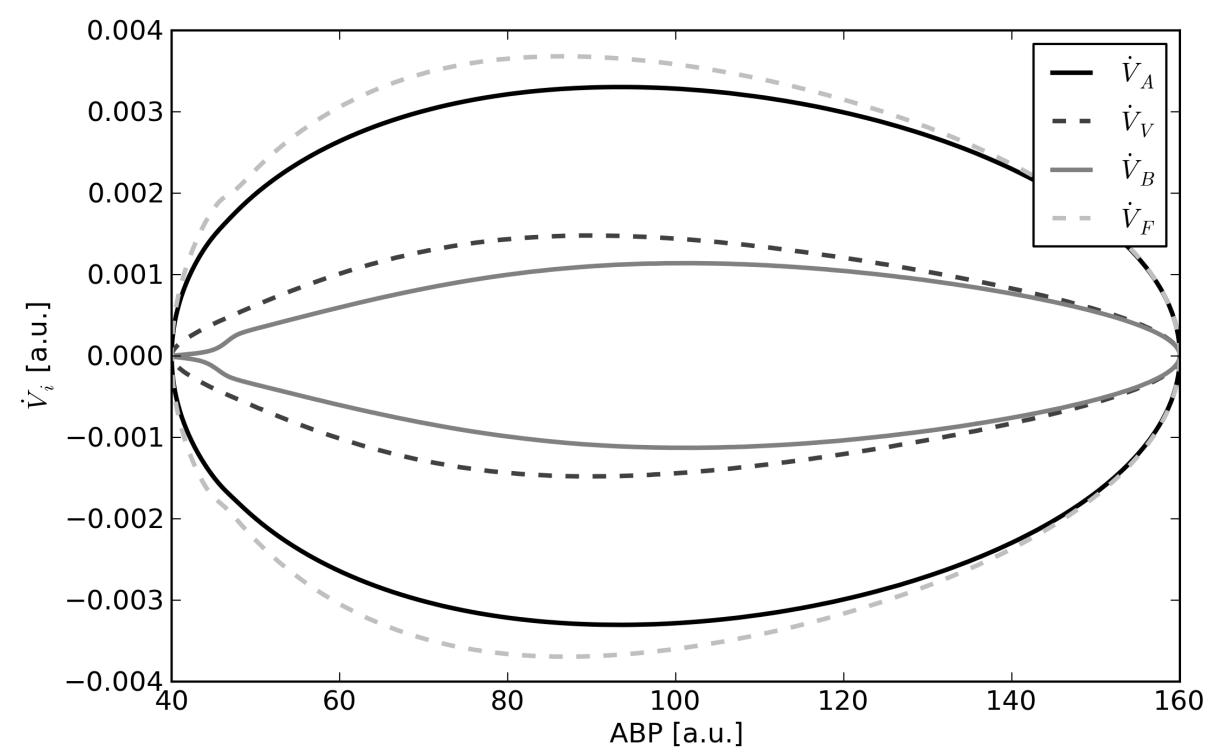

Figure 6.6: $\dot{V}_{i}$ against ABP with $\alpha=0.0$ and $V_{E}=0 \mathrm{ml}$ (sHOM; case 1).

seen in table 5.3 and 6.3. The volume of the brain compartment has the same maximal value in both scenarios, whereas its minimal value is slightly lower, as it is for the fluid compartment. The minimal value for the veins are around the same value, whereas the maximal value for the volume of the veins is higher in the sHOM case (about $7 \%$ ).

\begin{tabular}{l|r|r|r}
$\Delta$ Volume $[\mathrm{ml}]$ & $\min$ & $\max$ & range \\
\hline$\Delta V_{A}$ & -8.32 & 7.47 & 15.79 \\
$\Delta V_{B}$ & -2.52 & 2.04 & 4.56 \\
$\Delta V_{F}$ & -7.81 & 9.53 & 17.34 \\
$\Delta V_{V}$ & -3.25 & 2.86 & 6.11
\end{tabular}

Table 6.3: Ranges for the volume changes with $\alpha=0.0$ and $V_{E}=0 \mathrm{ml}$ (sHOM; case 1).

\subsubsection{Case 2: no swelling, autoregulation}

In the next scenario, the autoregulation mechanism is enabled. Figure 6.7 shows the simulated CBF of the bHOM and sHOM model for this case. The differences in $\mathrm{CBF}$ between both models is quite small: For ABP values above $100 \mathrm{mmHg}$, the $\mathrm{CBF}$ in the sHOM model is a little bit lower. As 
for values below $100 \mathrm{mmHg} \mathrm{ABP}$, the $\mathrm{CBF}$ in the sHOM case is a little bit higher. But the differences in CBF between both models can be neglected in general, as the deviation between both is smaller than $1 \%$. In the following sections, the differences between both models will be examined in more detail, but from the CBF graphs it can be assumed, that there will be no major variation between both models, due to the starling resistor.

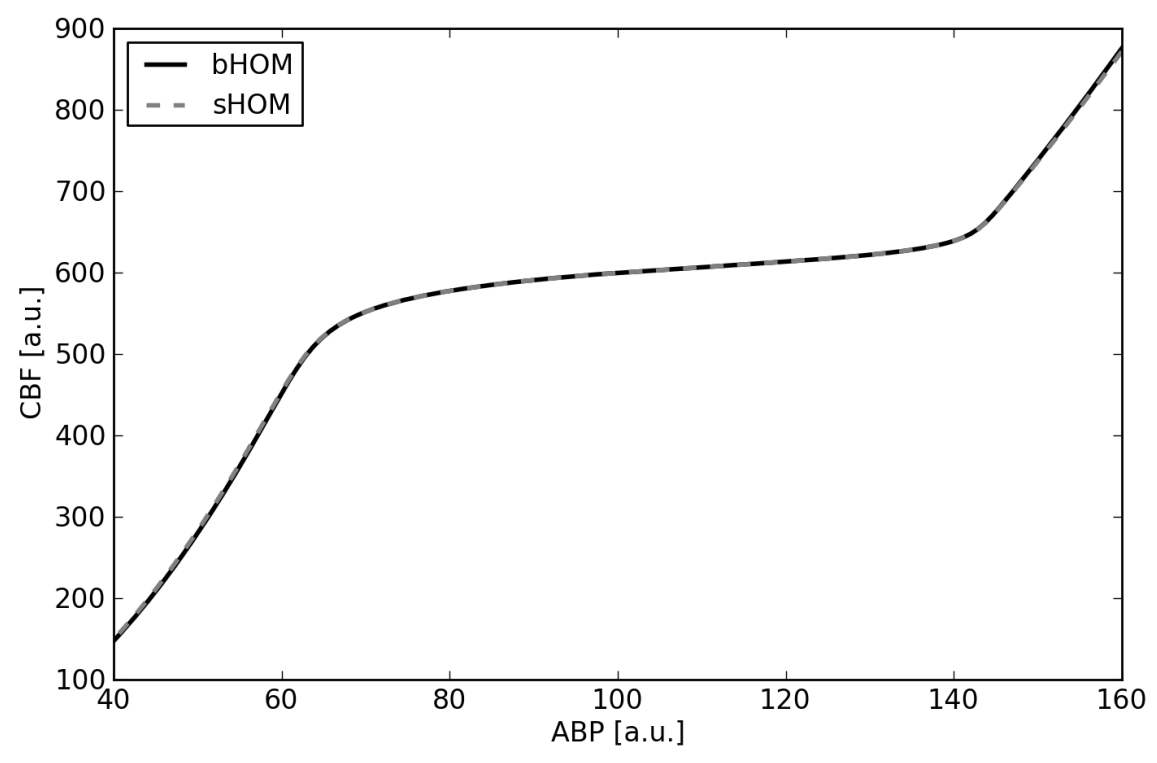

Figure 6.7: $\mathrm{CBF}$ against $\mathrm{ABP}$ with $\alpha=2.0$ and $V_{E}=0 \mathrm{ml}$ (bHOM and sHOM; case 2).

\section{Compliances}

The development of the compliances in both models is nearly identical, as it has been already in the former scenario, in which the autoregulation has been disabled. From a qualitative point of view, the graphs for the corresponding compliances between both models are the same. From a quantitative point of view, there are small differences: The compliance of the veins has a lower minimum and a higher maximum value in the sHOM case, both in a range of about $5 \%$. The same holds for the compliance of the brain tissue. But there, the difference is smaller than $1 \%$ between both.

As for the compliance of the arteries, there is no significant difference between both models and it can be considered equal. Table 6.4 summaries the important values for the compliances of the sHOM model. The corresponding values of the bHOM model can be found in table 5.4. 


\begin{tabular}{l|r|r|r} 
Compliance $\left[\frac{\mathrm{ml}}{\mathrm{mmHg}}\right]$ & $\min$ & $\max$ & range \\
\hline$C_{A B}$ & 0.085 & 0.315 & 0.230 \\
$C_{V B}$ & 0.341 & 0.891 & 0.550 \\
$C_{B}$ & 0.290 & 0.531 & 0.241
\end{tabular}

Table 6.4: Ranges for the compliances with $\alpha=2.0$ and $V_{E}=0 \mathrm{ml}$ (sHOM; case 2).

\section{Pressure}

As before, the development of the pressures in both models can be considered equal in a qualitative way. This behavior could be assumed from the corresponding CBF graphs, as both graphs are nearly equal and the CBF depends, aside from the resistance $R_{A C}$, on the pressure differences between arteries and capillaries.

From a qualitative point of view, the difference in the range of the pressure between both models is smaller than $1 \%$ for the arteries and veins. The difference for the range of the ICP is a little bit higher, around $3 \%$, but still in the same order of magnitude. Table 6.5 summarizes the pressure values for the sHOM model, whereas the corresponding values for the bHOM model can be found in table 5.5.

\begin{tabular}{l|r|r|r|r} 
Pressure $[\mathrm{mmHg}]$ & $\min$ & default & $\max$ & range \\
\hline$p_{A}$ & 40.00 & 100.00 & 160.00 & 120.00 \\
$p_{C}$ & 9.97 & 25.00 & 34.06 & 24.09 \\
$p_{V}$ & 7.84 & 15.00 & 19.53 & 11.69 \\
$p_{B}$ & 6.24 & 10.00 & 12.26 & 6.02
\end{tabular}

Table 6.5: Pressure ranges with $\alpha=2.0$ and $V_{E}=0 \mathrm{ml}$ (sHOM; case 2).

\section{Resistances}

Figure 6.8 and 6.9 shows the development of the resistances of the arteries and the veins in the time domain and against $\mathrm{ABP}$, respectively.

The development of the resistance of the arteries can be considered identical to the bHOM model, which could have been already deduced from the graphs of the $\mathrm{CBF}$, pressures and compliances.

The development of the veins is quite differently, when compared with the corresponding scenario of the bHOM model (section 5.2.2), but shows a similar behavior when compared with the simulations with disabled autoregulation, done in the previous section. The resistance of the veins remains 


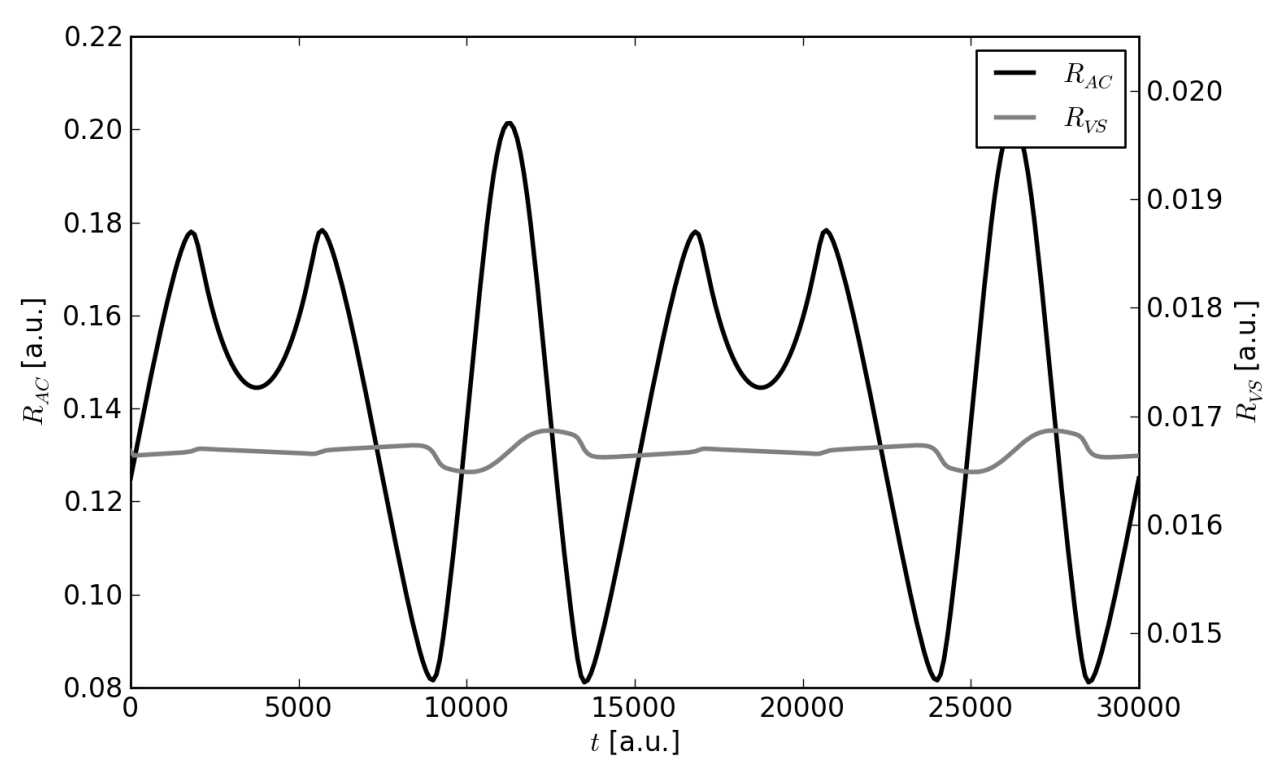

Figure 6.8: $R_{X Y}$ against time with $\alpha=2.0$ and $V_{E}=0 \mathrm{ml}$ (sHOM; case 2).

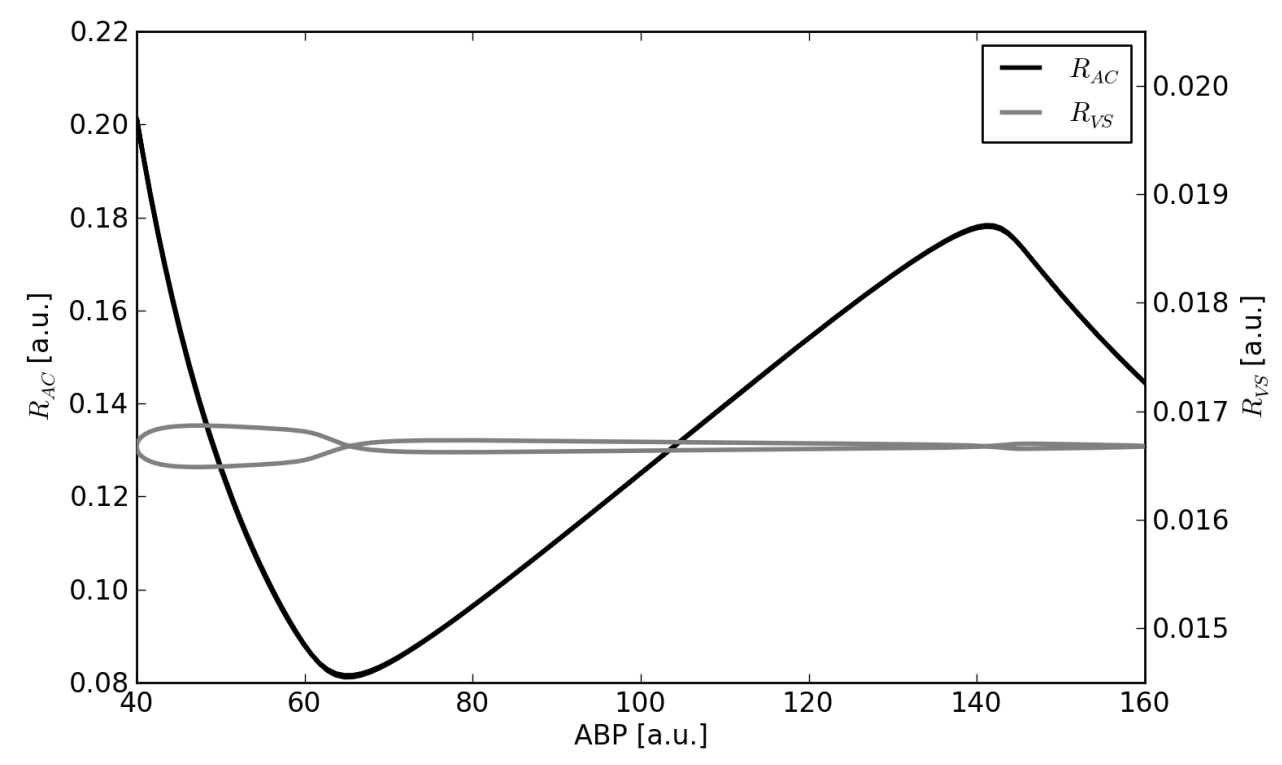

Figure 6.9: $R_{X Y}$ against $\mathrm{ABP}$ with $\alpha=2.0$ and $V_{E}=0 \mathrm{ml}$ (sHOM; case 2). 
nearly constant over the whole ABP range, whereas the absolute deviations are only $1 \%$ of the mean value for the vein pressure. ${ }^{\dagger}$

Therefore, the same conclusions can be drawn as in the previous scenario (compare section 6.1.1), namely, that the pressure inside the veins increases twice as fast as the ICP for increasing ABP.

\section{Volumes}

From the previous sections it is clear, that there will be not much difference in the volume development between both models, as can be seen in figure 6.10 (compare with fig. 5.22 for bHOM).

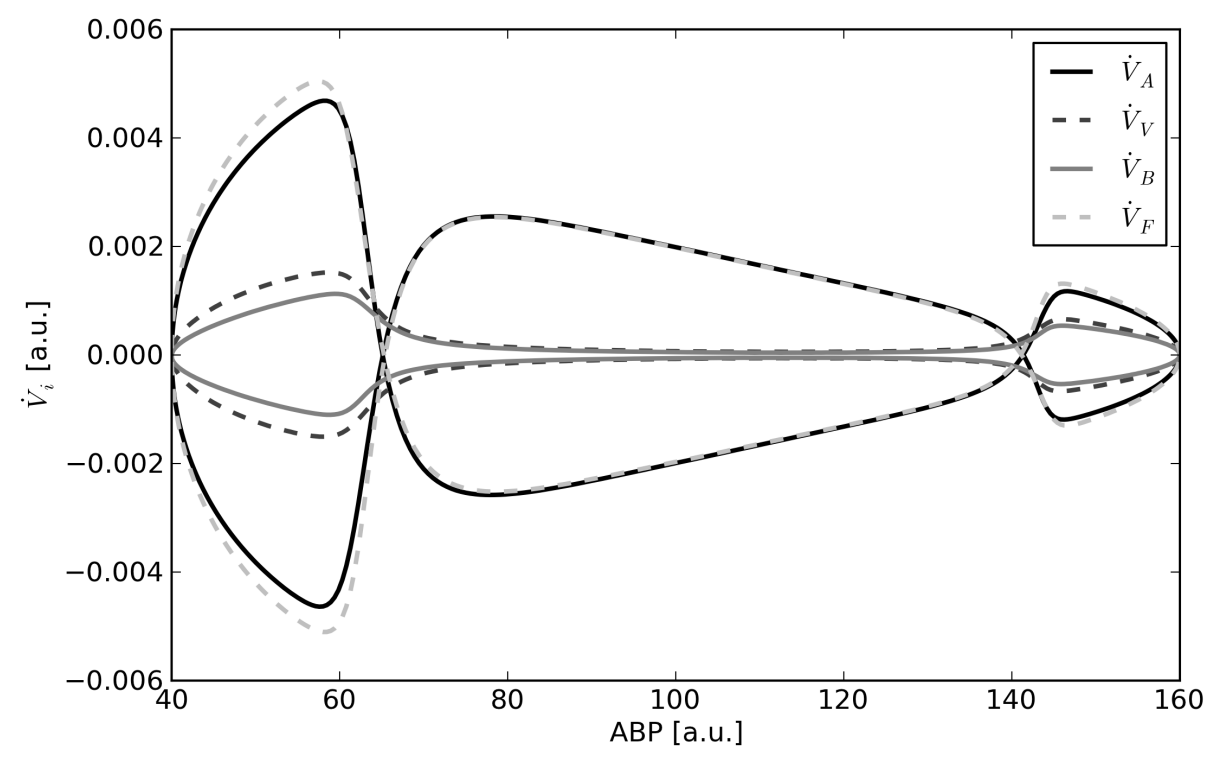

Figure 6.10: $\dot{V}_{i}$ against ABP with $\alpha=2.0$ and $V_{E}=0 \mathrm{ml}$ (sHOM; case 2).

Apart from the development of the resistance of the veins in the sHOM case, the development of the other quantities is more or less identical for both models. As also seen in the previous case, the different modeling of $R_{V S}$ does not have a big influence on the behavior of the model in general for the scenario, where there is no swelling of the brain.

\footnotetext{
${ }^{\dagger}$ The difference lies in the absence of the sharp bend, seen in case 1, where there is no autoregulation and the $\mathrm{ABP}$ values are below $47 \mathrm{mmHg}$.
} 


\subsection{Swelling}

Both simulations of the previous sections, namely without and with enabled autoregulation and no swelling, will now be repeated with the difference, that a swelling $\left(V_{E}=30 \mathrm{ml}\right)$ of the brain is modeled. From the previous chapter it is known, that with a swelling the pressures inside the compartments raise, when there is no cerebrospinal fluid (CSF) left to absorb. As the starling resistor and therefore the resistance of the veins is pressure dependent, here, the sHOM model should behave quite differently from the bHOM model. As before, here, $\Delta V_{F}=V_{F}$ and not $V_{F}-V_{F n}$ is plotted, due to the swelling.

\subsubsection{Case 3: swelling, no autoregulation}

Taking a look at the CBFs of the simulations with a swelling and disabled autoregulation for the bHOM and the sHOM model, which are shown in figure 6.11, the difference in the development, which was assumed, can quite clearly be seen.

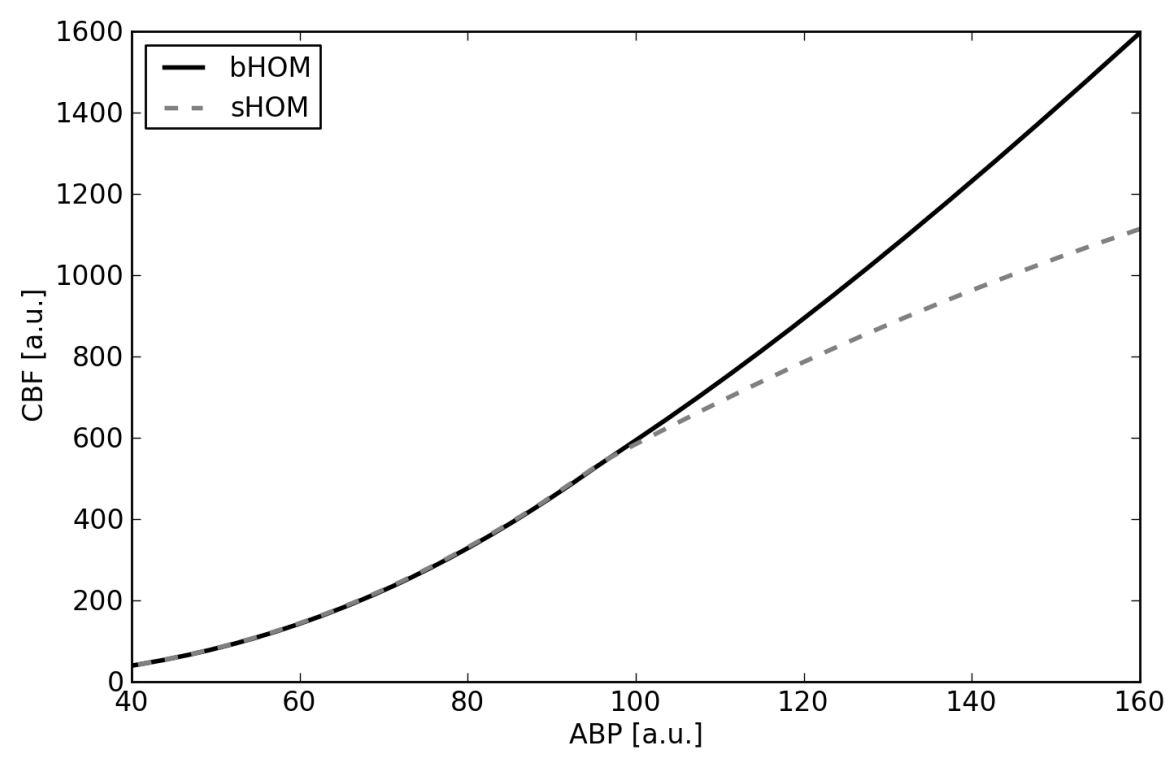

Figure 6.11: $\mathrm{CBF}$ against $\mathrm{ABP}$ with $\alpha=0.0$ and $V_{E}=30 \mathrm{ml}$ (bHOM and sHOM; case 3$)$.

For ABP values below $95 \mathrm{mmHg}$, both curves are practically identical, as in case 1. But above around $95 \mathrm{mmHg}$, the curves develop differently. Where the $\mathrm{CBF}$ of the bHOM model raises linearly in a first order approximation, the slope of the $\mathrm{CBF}$ for the sHOM model increases slower with raising ABP. 
The reason for this difference lies in the different pressure developments of the compartments, when the CSF is nearly completely depleted, as will be discussed in the next section.

\section{Pressure}

The development of the pressures can be seen in figure 6.12. Compared to the bHOM model (compare fig. 5.24), there are two obvious differences: First, the range of the pressures differs and second, the sharp bend, which was only obvious in the ICP of the bHOM case, can now be seen in all pressure curves.

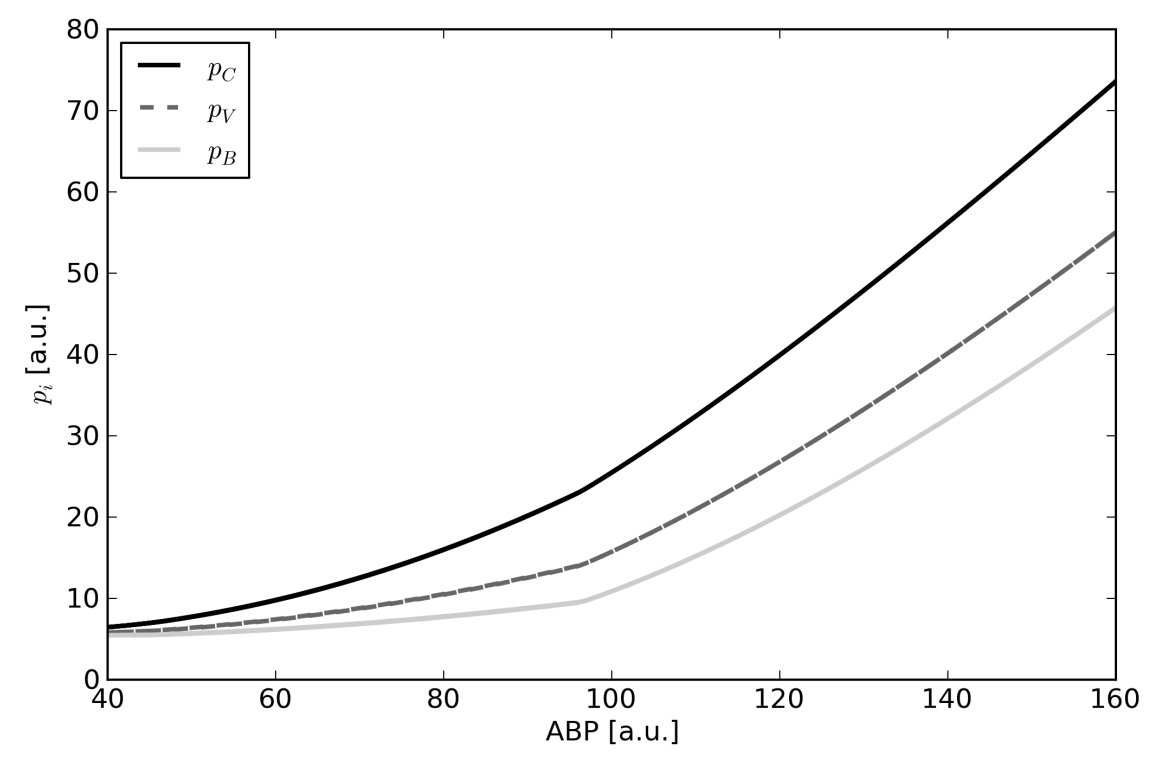

Figure 6.12: $p_{i}$ against $\mathrm{ABP} \alpha=0.0$ and $V_{E}=30 \mathrm{ml}$ (sHOM; case 3 ).

For ABP values below around $95 \mathrm{mmHg}$, the pressure curves are similar in both models. But around an ABP value of $95 \mathrm{mmHg}$, there is a bend in all pressure curves, which is not as sharp for the pressure inside the capillaries and veins as it is for the ICP, but still visible. Above a raising ABP of $95 \mathrm{mmHg}$, the pressure curves of all compartments have a steeper slope, when compared to the bHOM case.

The difference is the smallest for the pressure inside the capillaries. There, the maximal value for $p_{C}$ differs about $12.79 \mathrm{mmHg}$, which can be seen in table $6.6(\mathrm{sHOM})$ and table $5.6(\mathrm{bHOM})$. For the pressure inside the veins, the difference lies at $21.02 \mathrm{mmHg}$ and for ICP at $13.41 \mathrm{mmHg}$ between both models. Considering the standard values for $p_{V}=15 \mathrm{mmHg}$ 
and $p_{B}=10 \mathrm{mmHg}$, the increase of pressure inside the veins and ICP is quite similar, contrary to the bHOM model. There, the ICP increased faster than $p_{V}$.

\begin{tabular}{l|r|r|r|r} 
Pressure $[\mathrm{mmHg}]$ & min & default & max & range \\
\hline$p_{A}$ & 40.00 & 100.00 & 160.00 & 120.00 \\
$p_{C}$ & 6.45 & 25.00 & 73.60 & 67.15 \\
$p_{V}$ & 5.80 & 15.00 & 55.04 & 49.24 \\
$p_{B}$ & 5.47 & 10.00 & 45.76 & 40.29
\end{tabular}

Table 6.6: Pressure ranges with $\alpha=0.0$ and $V_{E}=30 \mathrm{ml}$ (sHOM; case 3).

Another difference is, that for the sHOM model, there is no drop in capillary pressure, as seen in the corresponding case of the bHOM model (compare section 5.3.1). Here, all pressures show an increase for raising $\mathrm{ABP}$ values higher than $95 \mathrm{mmHg}$.

Although, the pressure inside the capillaries increases, when compared to case 1, this should not be enough to cause the drop in CBF, seen in figure 6.11. Therefore, the behavior of the resistance $R_{A C}$ should also be different from case 1.

\section{Resistance}

The course of the resistances $R_{A C}$ and $R_{V S}$ are depicted in figure 6.13. The resistance of the arteries is quite similar in the bHOM and sHOM model. The only difference lies in a smaller decrease from a raising ABP around $95 \mathrm{mmHg}$ in the sHOM case. The minimal value for $R_{A C}$ is $0.078 \frac{\mathrm{mmHg}}{\mathrm{ml} / \mathrm{min}}$ contrary to $0.051 \frac{\mathrm{mmHg}}{\mathrm{ml} / \mathrm{min}}$ in the corresponding simulations with the bHOM model. Therefore, the resistance in the arteries is bigger for ABP values above $95 \mathrm{mmHg}$ than in the bHOM model, which together with the increased pressure inside the capillaries is responsible for the drop of $\mathrm{CBF}$ seen in fig. 6.11. The reason for the increase in $R_{A C}$ lies in the increased ICP, which can be deduced from eq. 3.6.

The development of $R_{V S}$ is similar as in case 1 and for ABP values below $95 \mathrm{mmHg}$. But here, from this point onwards there is a sharp bend upwards, which is similar to the development already seen in the pressure curves. With an increasing ABP above $95 \mathrm{mmHg}$, the resistance of the veins raises linearly, in a first order approximation, with increasing $\mathrm{ABP}$, until it reaches its maximum at the maximal $\mathrm{ABP}$ value, which is about three times bigger than for case 1 .

This behavior confirms the assumption from the previous section, in which it was stated, that the development of the pressure inside the veins and ICP for ABP values higher than $95 \mathrm{mmHg}$ is similar, which follows from 


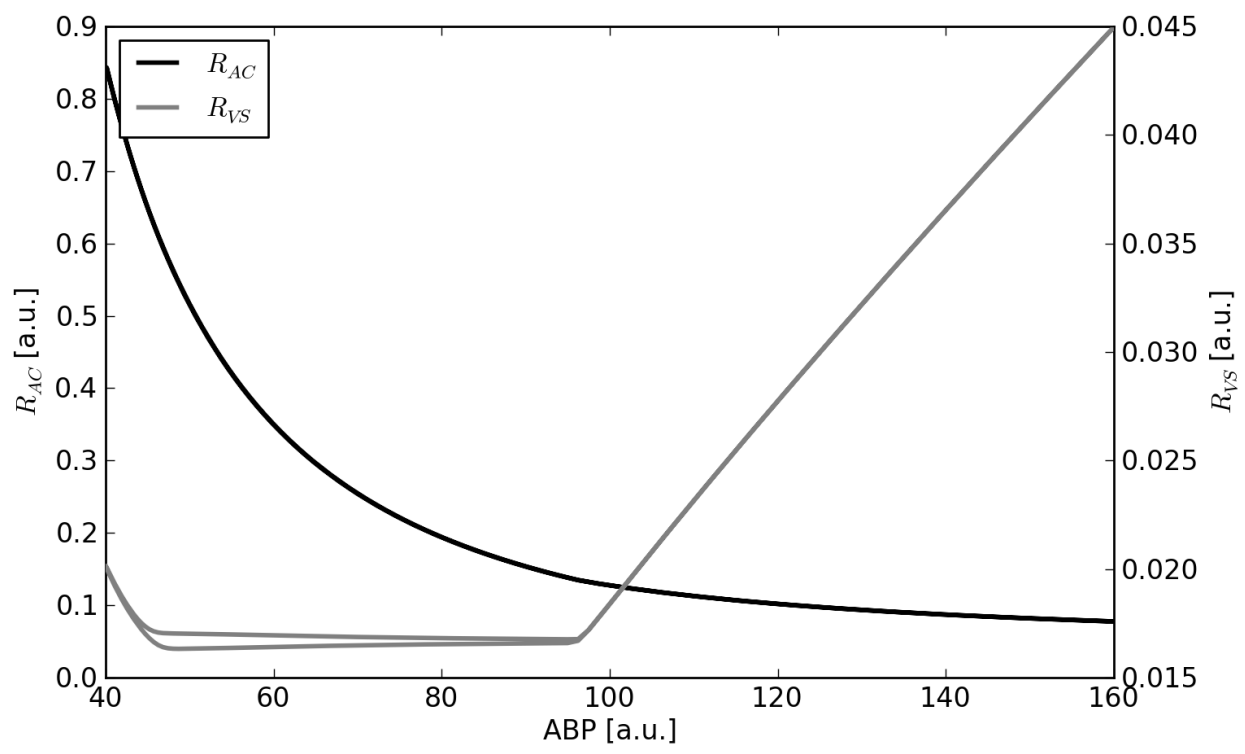

Figure 6.13: $R_{X Y}$ against ABP with $\alpha=0.0$ and $V_{E}=30 \mathrm{ml}$ (sHOM; case $3)$.

eq. 6.1 .

\section{Compliance}

The graphs for the compliances are shown in figure 6.14. As the autoregulation is disabled, the compliance of the arteries remains constant over the whole range of the ABP.

The development of the compliance of the brain tissue is, from a qualitative point of view, similar to the corresponding simulation of the bHOM model. The difference lies in a steeper decrease for ABP values above $97 \mathrm{mmHg}$ and therefore a slightly lower minimal value of $C_{B}$ (compare table 6.7 and 5.8).

But the development for $C_{V B}$ is completely different for ABP values above $97 \mathrm{mmHg}$, when compared with the corresponding simulation of the bHOM model. Whereas there, the compliance of the veins raises with increasing ABP, here, the compliance decreases (compare fig. 5.26). The difference between both developments lies in the different pressure development. Whereas in the bHOM model, the increase in pressure inside the veins is bigger than of the ICP, in the sHOM model both pressure curves have a similar slope. As the increase in $p_{V}$ is slightly higher than the increase in $\mathrm{ICP}$, the graph decreases with increasing ABP. 


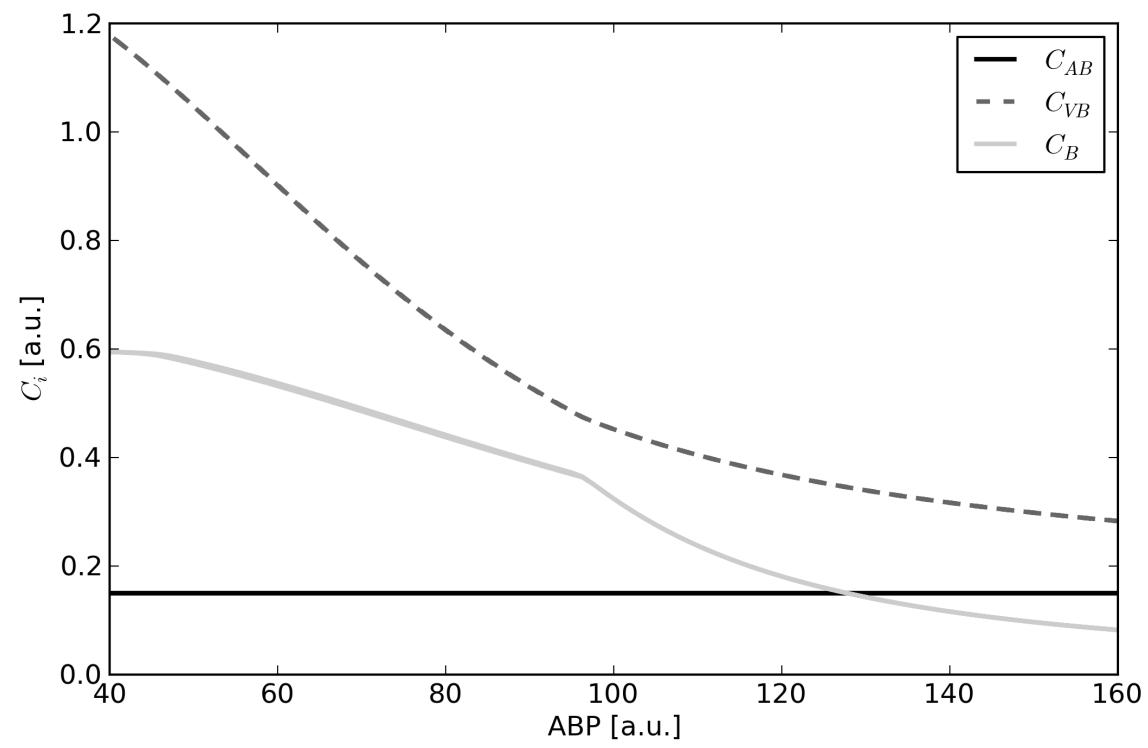

Figure 6.14: $C_{i}$ against ABP with $\alpha=0.0$ and $V_{E}=30 \mathrm{ml}$ (sHOM; case 3).

Due to the different development of the compliances and pressures, the volumes of the compartments should have a significantly different development, which will be shown in the next section.

\begin{tabular}{l|r|r|r} 
& \multicolumn{3}{|c}{$\mathrm{ABP}$} \\
Compliance $\left[\frac{\mathrm{ml}}{\mathrm{mmHg}}\right]$ & $40 \mathrm{mmHg}$ & $97 \mathrm{mmHg}$ & $160 \mathrm{mmHg}$ \\
\hline$C_{A B}$ & 0.15 & 0.15 & 0.15 \\
$C_{V B}$ & 1.18 & 0.47 & 0.28 \\
$C_{B}$ & 0.59 & 0.36 & 0.08
\end{tabular}

Table 6.7: Ranges for the compliances with $\alpha=0.0$ and $V_{E}=30 \mathrm{ml}$ (sHOM; case 3$)$.

\section{Volumes}

In figure 6.15 and 6.16 , the development of the volumes of each compartment can be seen. The first graphs shows $\Delta V_{i}$ against $\mathrm{ABP}$, whereas in the second graph, the deviations of the volumes $\dot{V}_{i}$ are shown.

As long as there is CSF left to absorb, the graphs do not differ from the simulations without a swelling and are therefore similar to the corresponding 


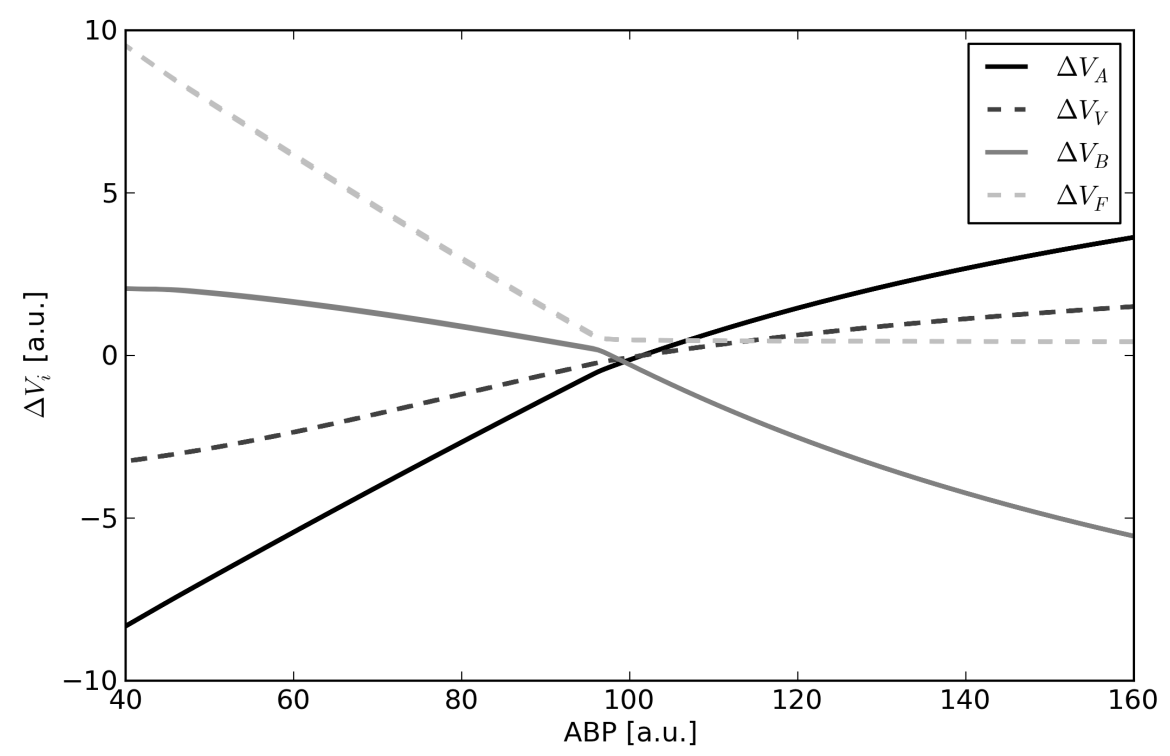

Figure 6.15: $\Delta V_{i}$ against $\mathrm{ABP}$ with $\alpha=0.0$ and $V_{E}=30 \mathrm{ml}$ (sHOM; case $3)$.

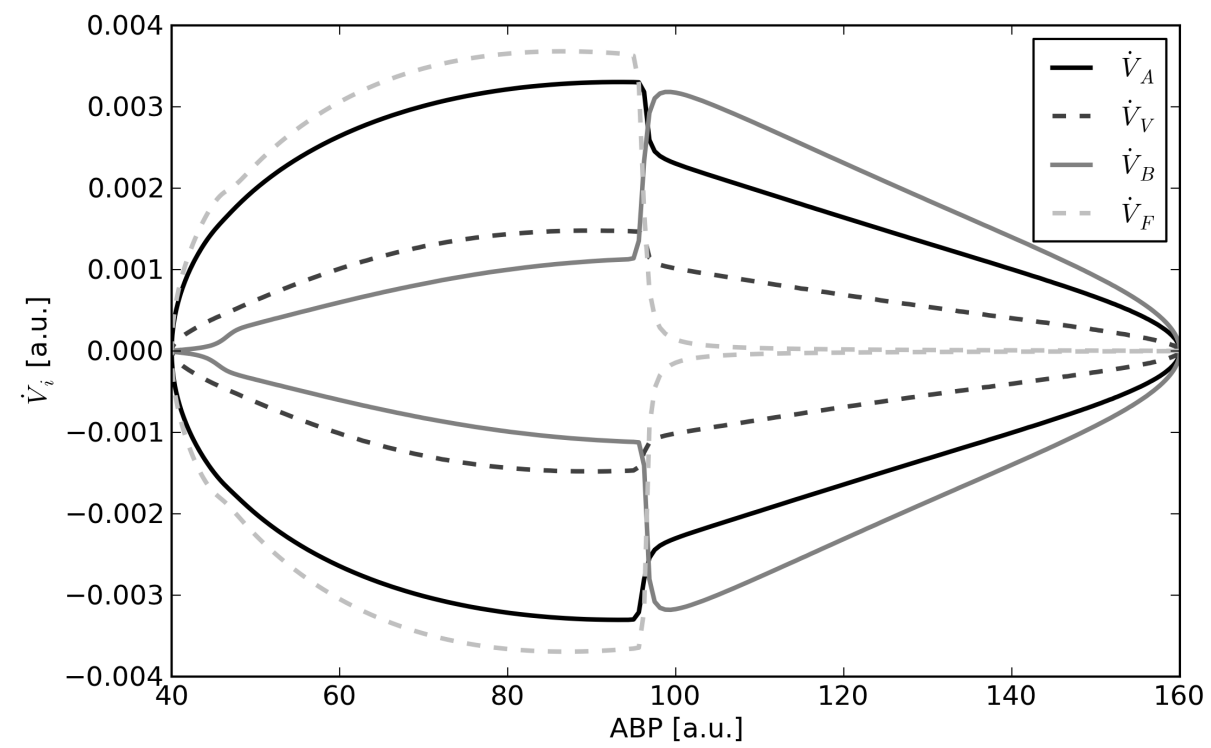

Figure 6.16: $\dot{V}_{i}$ against $\mathrm{ABP}$ with $\alpha=0.0$ and $V_{E}=30 \mathrm{ml}$ (sHOM; case 3 ). 
simulations with the bHOM model. But the behavior differs a lot, compared to the bHOM model, when there is no CSF left to absorb.

The course of the depletion of the CSF compartment can be considered equal for both models, which can be seen in fig. 5.27 and 6.15 .

Due to the higher ICP, the volume increase of the arteries is much smaller than in the bHOM model. Contrary to the nearly linear behavior of $\Delta V_{A}$ in the bHOM model, here, $\Delta V_{A}$ gets even flatter with increasing ABP.

The volume development of the veins has changed completely. Whereas in the former simulation, the change in volume was negative for ABP values above $97 \mathrm{mmHg}$, here, the volume increases. This behavior is more similar to the simulations done with the bHOM model for no autoregulation and no swelling. There, the development of the pressures and compliances has shown a similar behavior (compare section 5.2.1, and fig. 5.9 for volume development).

As both, the volume of the veins and arteries, increase, the volume of the brain tissue has to decrease, as there is no CSF left to absorb. This is responsible for the big increase of ICP.

In table 7.7 the extremal values for the minima and maxima of the volume changes in each compartment are summarized.

\begin{tabular}{l|r|r|r}
$\Delta$ Volume $[\mathrm{ml}]$ & $\min$ & $\max$ & range \\
\hline$\Delta V_{A}$ & -8.32 & 3.63 & 11.95 \\
$\Delta V_{B}$ & -5.56 & 2.05 & 7.61 \\
$\Delta V_{F}$ & 0.45 & 9.53 & 9.08 \\
$\Delta V_{V}$ & -3.25 & 1.51 & 4.76
\end{tabular}

Table 6.8: Ranges for volume changes with $\alpha=0.0$ and $V_{E}=30 \mathrm{ml}$ (sHOM; case 3$)$.

\subsubsection{Case 4: swelling, autoregulation}

Now, the last simulation of the previous chapter is repeated with the sHOM model, in which both a swelling is simulated and the autoregulation mechanism is enabled. From the previous sections and chapter it can be assumed, that at least below $100 \mathrm{mmHg}$ there will be an interval, in which there is no CSF left to absorb. In this ABP range, all pressures should have increased values, when compared to the same simulation, done with the bHOM model. Also, the volume development inside this range should look differently.

In figure 6.17 the development of the $\mathrm{CBF}$ is shown for both the bHOM and sHOM model. Contrary to the bHOM model, here, the drop in CBF is much more obvious. There, only a small drop in the CBF between an ABP of approx. $50 \mathrm{mmHg}$ and $85 \mathrm{mmHg}$ can be seen in case 4 , when compared to 
case 2. Also, the development of the CBF inside this interval has a different form and appears to be linear in a first approximation.

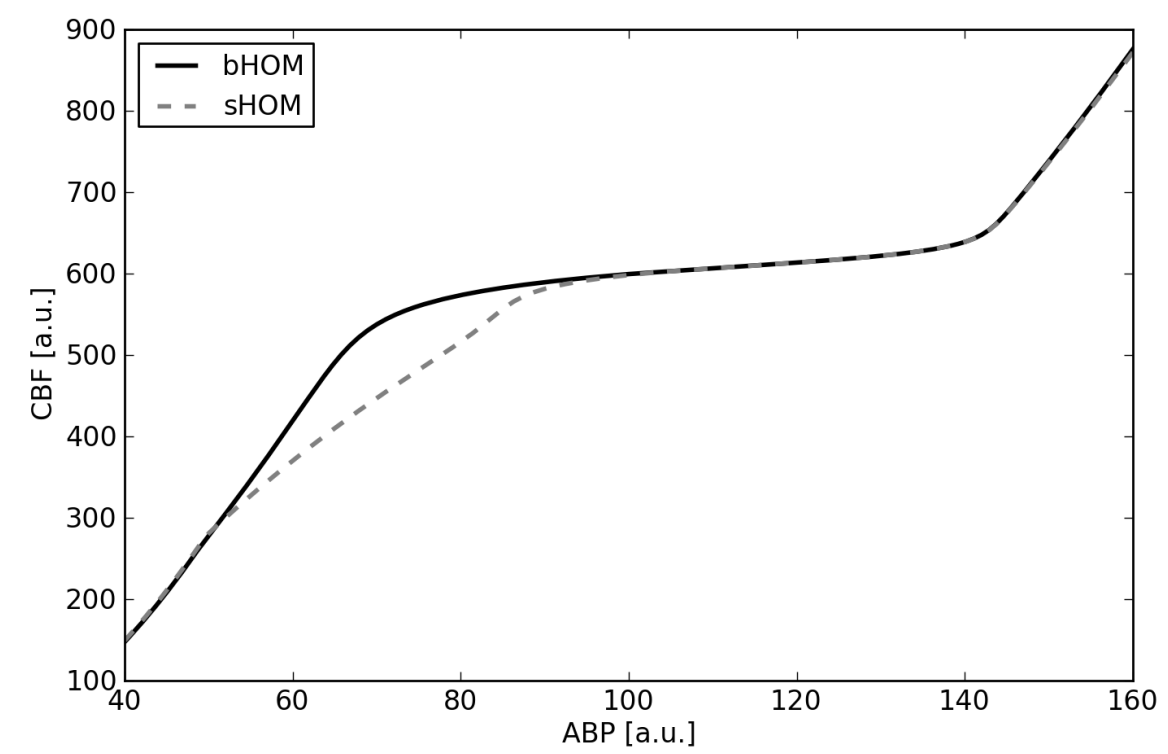

Figure 6.17: $\mathrm{CBF}$ against $\mathrm{ABP}$ with $\alpha=2.0$ and $V_{E}=30 \mathrm{ml}$ (bHOM and sHOM; case 4).

\section{Pressures}

As in the previous simulations for the sHOM model with disabled autoregulation and a swelling, a change in pressure can be seen in all compartments. The corresponding graphs are depicted in figure 6.18. At an increasing ABP of around $50 \mathrm{mmHg}$ all pressures values show an advanced increase, when compared to the simulations with enabled autoregulation and no swelling. This increase reaches its maximum around an $\mathrm{ABP}$ of $80 \mathrm{mmHg}$, from where it drops with increasing $\mathrm{ABP}$ and vanishes at around an $\mathrm{ABP}$ of $105 \mathrm{mmHg}$. From there onwards, the pressure development takes a similar course as in the no swelling case.

Between an ABP of $50 \mathrm{mmHg}$ and $105 \mathrm{mmHg}$, the increase for the pressure inside the capillaries is a little bit smaller than for the pressure inside the other compartments, with a maximum additional value of about $14 \mathrm{mmHg}$. For the pressure inside the veins and brain tissue, the increase has approx. the same amount and is around $15-16 \mathrm{mmHg}$. 


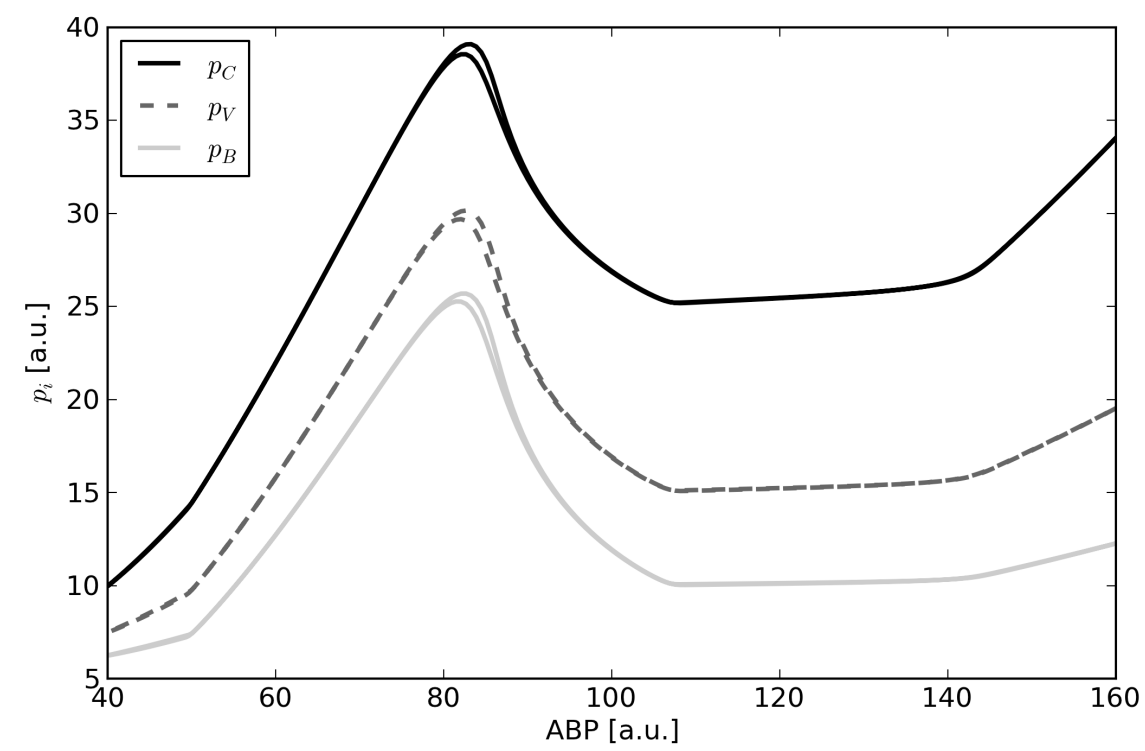

Figure 6.18: $p_{i}$ against ABP with $\alpha=2.0$ and $V_{E}=30 \mathrm{ml}$ (sHOM; case 4).

\section{Resistances}

As for the development of the resistance of the arteries and veins, in this case both curves differ from the similar simulations of the sHOM model without a swelling. This is different from the same simulations with the bHOM model, in which there was only a significant difference between the development of the resistance of the veins, but not for the arteries. The corresponding graphs are shown in figure 6.19.

An explanation for the resistance development of the veins can easily be given, with the knowledge of the previous chapter and sections: Outside of the interval, where the CBF of this scenario is identical to the corresponding simulation with the sHOM model and no swelling, the behavior of the pressure curves in both scenarios are similar and therefore, the development of $R_{V S}$ in both scenarios has to be similar, too, and is therefore nearly constant. Inside the interval, the development of the pressure inside the veins and ICP has a similar trend, which results in the increase of $R_{V S}$, which has a similar shape as the additional increase of ICP and pressure inside the veins. The same behavior could be seen in the simulations with the sHOM model, in which there was a swelling and no autoregulation. There, the reason for this behavior was the depletion of the CSF compartment. Therefore, it is safe to assume, that here, the cause of this peak is the same, namely, the total absorption of CSF. 


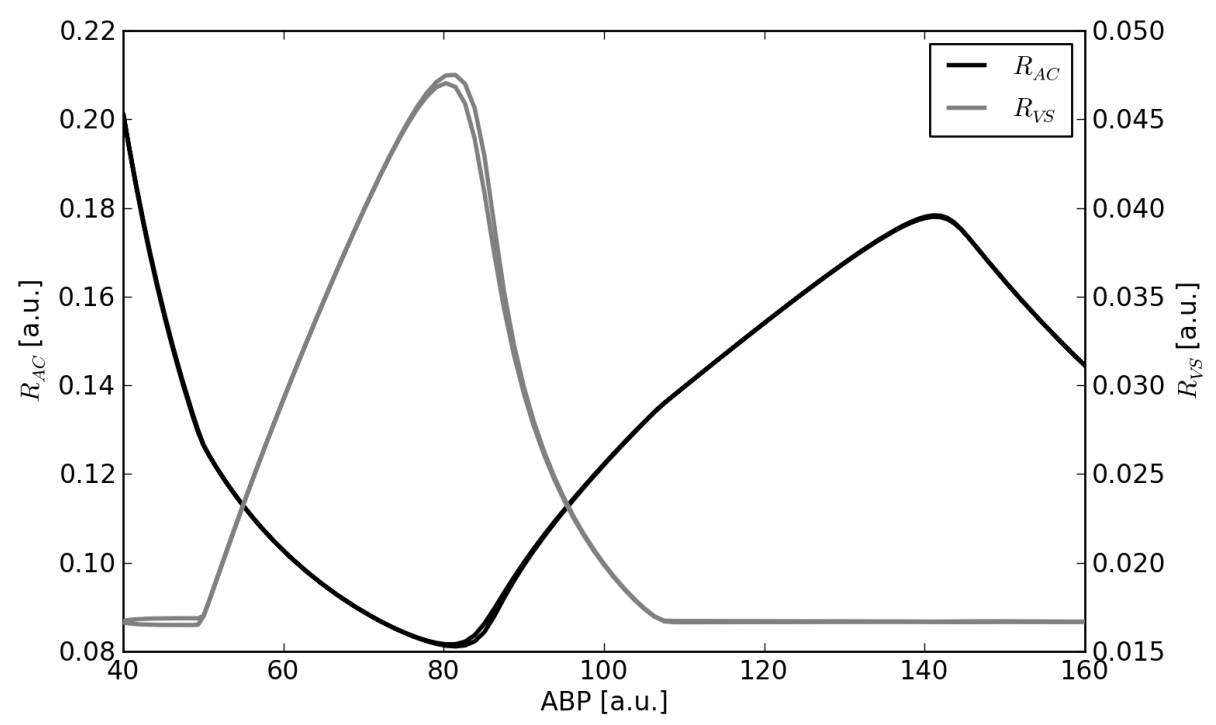

Figure 6.19: $R_{X Y}$ against $\mathrm{ABP}$ with $\alpha=2.0$ and $V_{E}=30 \mathrm{ml}$ (sHOM; case $4)$.

As for the development of the resistance of the arteries: For ABP values above approx. $105 \mathrm{mmHg}$, the pressure development inside the compartments is equal to the no swelling case and therefore identical. For decreasing $\mathrm{ABP}$ values below $105 \mathrm{mmHg}$, the pressure inside of each compartment raises, until an $\mathrm{ABP}$ of approx. $83 \mathrm{mmHg}$ is reached. This raise in pressure inside the capillaries is compensated by the autoregulations mechanism and therefore a change in their compliance and with it, the dilation of the arteries. But due to the increase in the volume of the arteries, the pressure inside the brain tissue will increase further, which will increase the pressure inside the veins. This ultimately results in an increase of the pressure inside the capillaries, which results in a premature maximal active dilation of the arteries. This can be seen in the resistance curve, where the minimal arteries resistance is already reached at around an $\mathrm{ABP}$ of $83 \mathrm{mmHg}$, contrary to the previous scenario, where the minimal value was around an $\mathrm{ABP}$ of $60 \mathrm{mmHg}$.

For further decreasing ABP values below $83 \mathrm{mmHg}$, the autoregulation mechanism has reached its maximum. As there is no more active contribution to the volume change of the arteries, brain pressure will decrease with decreasing ABP, as will the pressure for the other compartments, which ultimately results in an increase of $R_{A C}$. At around $50 \mathrm{mmHg}$, the depletion of the CSF compartment has stopped and the former development of the resistances for the no swelling case can be seen. 


\section{Compliances}

The graphs of the compliances are shown in figure 6.20. Between an ABP of approx. $50 \mathrm{mmHg}$ and $105 \mathrm{mmHg}$, there is no CSF left to absorb, which can be deduced from the graph of the compliance of the brain tissue. Outside this segment, in which there is still CSF left to absorb, the graphs are identical to the previous simulations for the sHOM model with enabled autoregulation and no swelling and for this reason are not discussed (compare section 6.1.2 for discussion).

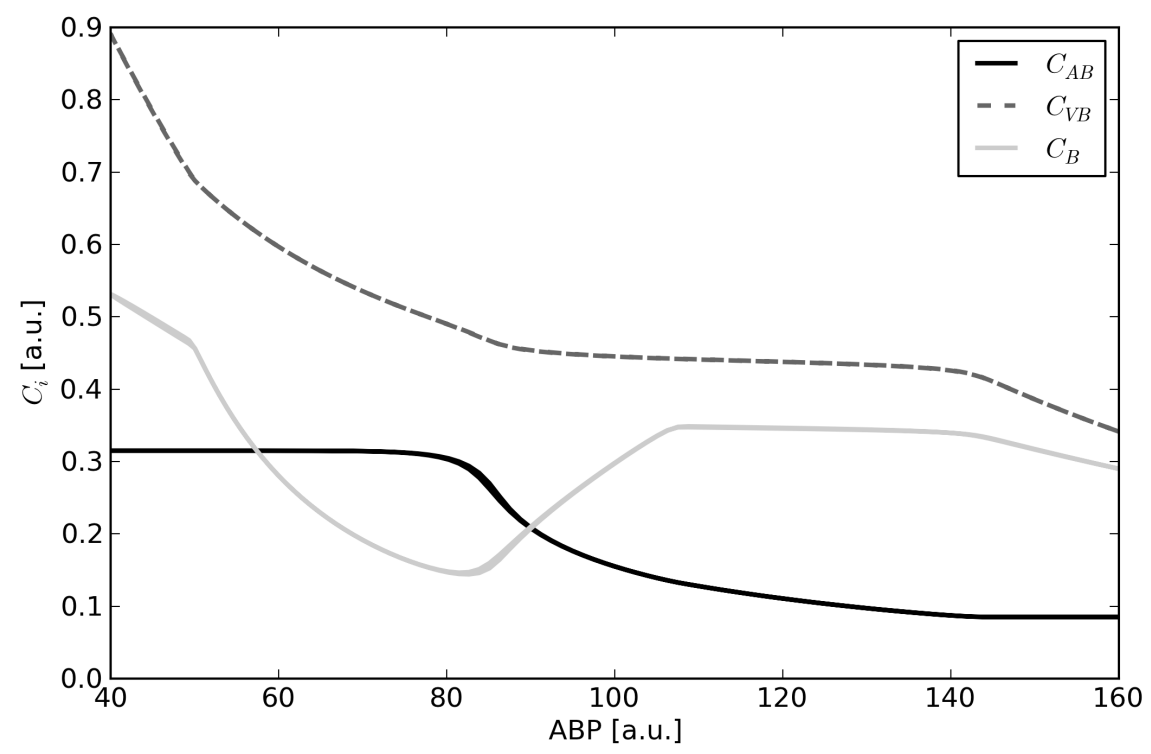

Figure 6.20: $C_{i}$ against ABP with $\alpha=2.0$ and $V_{E}=30 \mathrm{ml}$ (sHOM; case 4).

Therefore, the segment of interest is between an ABP of approx. $50 \mathrm{mmHg}$ and $105 \mathrm{mmHg}$. This segment can be divided into two subsegments for discussion, namely:

Segment 1: outside the autoregulation range $(\mathrm{ABP} \in[50,83][\mathrm{mmHg}])$

Segment 2: inside the autoregulation range $(\mathrm{ABP} \in[83,105][\mathrm{mmHg}])$

As it has already been mentioned in the previous section, the autoregulation range is reduced, with its new lower limit at an $\mathrm{ABP}$ of about $83 \mathrm{mmHg}$. This limit also marks the maximum of the compliance of the arteries and with it, the lower end of segment 2 , from where on the arteries cannot actively change the compliance with decreasing ABP anymore and is from there on at its maximum value and constant, which describes the behavior in segment 1. 
The development of the compliance of the brain tissue is similar to the corresponding simulation of the bHOM model. Primarily, there are two differences between both models. First, the minimal value for $C_{B}$ is much smaller, due to the increased pressures and second, due to the different lower limit of the autoregulation mechanism, the minimum of $C_{B}$ has moved to the lower limit of the $\mathrm{ABP}$ of the corresponding extremal autoregulation point. This also marks the lower end of segment 2 and the upper end of segment 1.

The development of the compliance of the veins shows a different development for both models. Inside segment 2 , the compliance $C_{V B}$ remains nearly constant, which is the behavior seen in case 2, where there was no swelling simulated. Outside segment 2 and therefore inside segment 1, there is an increase of $C_{V B}$ with decreasing $\mathrm{ABP}$, which develops more linearly than in case 2. It is always above the values of $C_{V B}$ in case 2 , until it reaches the end of segment 1 , from where on the former behavior is reproduced, as the CSF compartment is no longer depleted. In the bHOM model, $C_{V B}$ had a local maximum between its corresponding segments, due to the different pressure developments for $p_{V}$ and $p_{B}$. Here, as $p_{V}$ and $p_{B}$ have a similar shape, this peak is absent. From this it can be deduced, that the development of pressure inside the veins and ICP is similar in segment 2, but $p_{V}$ drops more rapidly than ICP in segment 1 with decreasing ABP, which is exactly what can be seen in figure 6.18 .

\section{Volumes}

The normalized volume development $\Delta V_{i}$ of each compartment is shown in figure 6.21. From a qualitative point of view, the volume developments for the corresponding simulations for both models can be considered equal for the case, where there is still CSF left to absorb. The development of the CSF compartment itself can be assumed to be identical to the same simulation with the bHOM model. When the CSF compartment is depleted, the other graphs differ in the following way.

Due to the different lower autoregulation point, the arteries reach their maximal value at the new extremal autoregulation point at about an ABP of $83 \mathrm{mmHg}$, from where on the volume decreases approximately linearly with decreasing ABP until it reaches the lower end of segment 1.

The same holds for the volume development of the brain tissue. The minimal value has moved to the new lower autoregulation point and the trend inside segment 1 and 2 has changed accordingly, as long as the CSF compartment is depleted. Also, the minimal value is lower than in the former scenario.

Contrary to the bHOM model, the volume of the veins increases monotonically with increasing $\mathrm{ABP}$ over the whole $\mathrm{ABP}$ range. This change in behavior of the veins was already discussed in case 2 of the sHOM model 


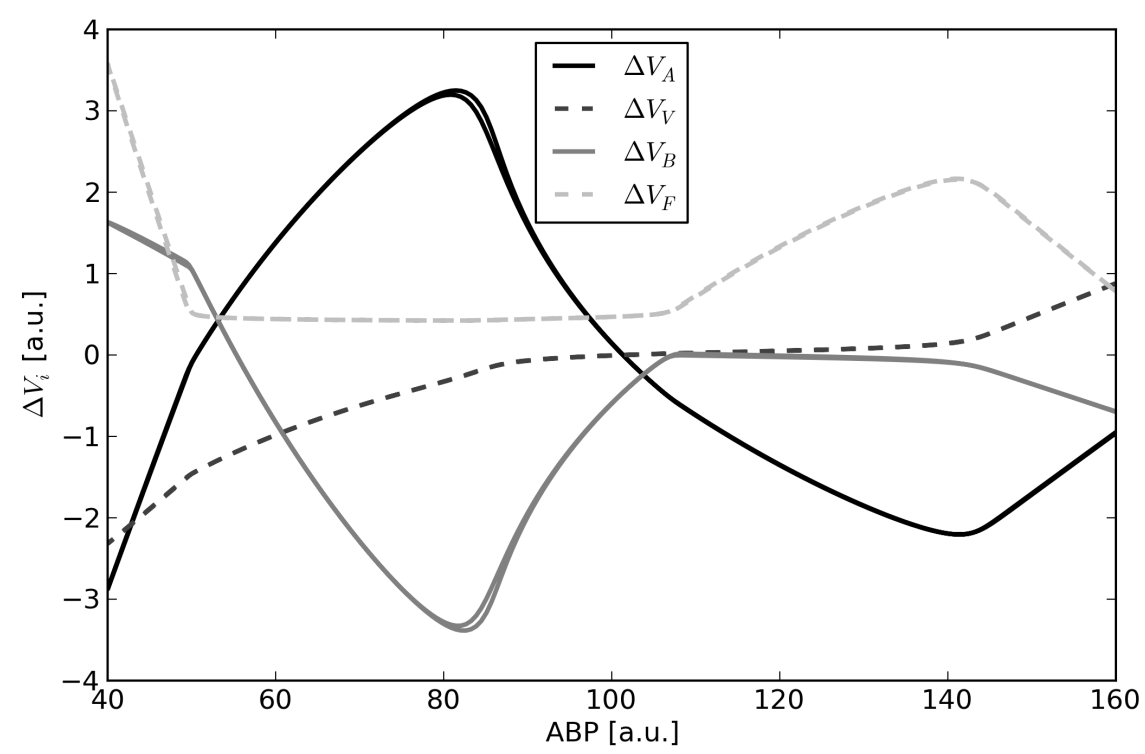

Figure 6.21: $\Delta V_{i}$ against $\mathrm{ABP}$ with $\alpha=2.0$ and $V_{E}=30 \mathrm{ml}$ (sHOM; case 4).

(compare section 6.1.2 and therefore, fig. 5.20).

For a more quantitative explanation, the deviations $\dot{V}_{i}$ can be used, which are depicted in figure 6.22 (against time) and 6.23 (against ABP). Here, the more complicated development of the volumes can be seen. As before, the graphs can be considered identical with case 2 outside the interval, in which the CSF compartment is depleted (compare section 6.1.2 and therefore, fig. 5.22).

When compared directly with the corresponding simulations of the basic model (see fig. 5.35), the deviations are comparable outside of segment 1 and 2 , but are totally different inside.

The development of $\dot{V}_{i}$ in segment 1 differs mainly in two points: First, the deviations increase/decrease monotonically, when the endpoints of this segment are neglected. There are no local extrema as in the volume development of the bHOM model. And second, the absolute deviation of the volume of the brain tissue is bigger than for the arteries.

But the main difference can be seen in segment 2. Here the change of volume of the arteries is mostly compensated by the inversed volume change of the brain tissue. The volume of the veins remains constant most of the time. Its absolute deviation increases for decreasing ABP values around $90 \mathrm{mmHg}$ and reaches an extremum between segment 1 and 2, from where on it remains nearly constant again, until it reaches the end of segment 1 . 


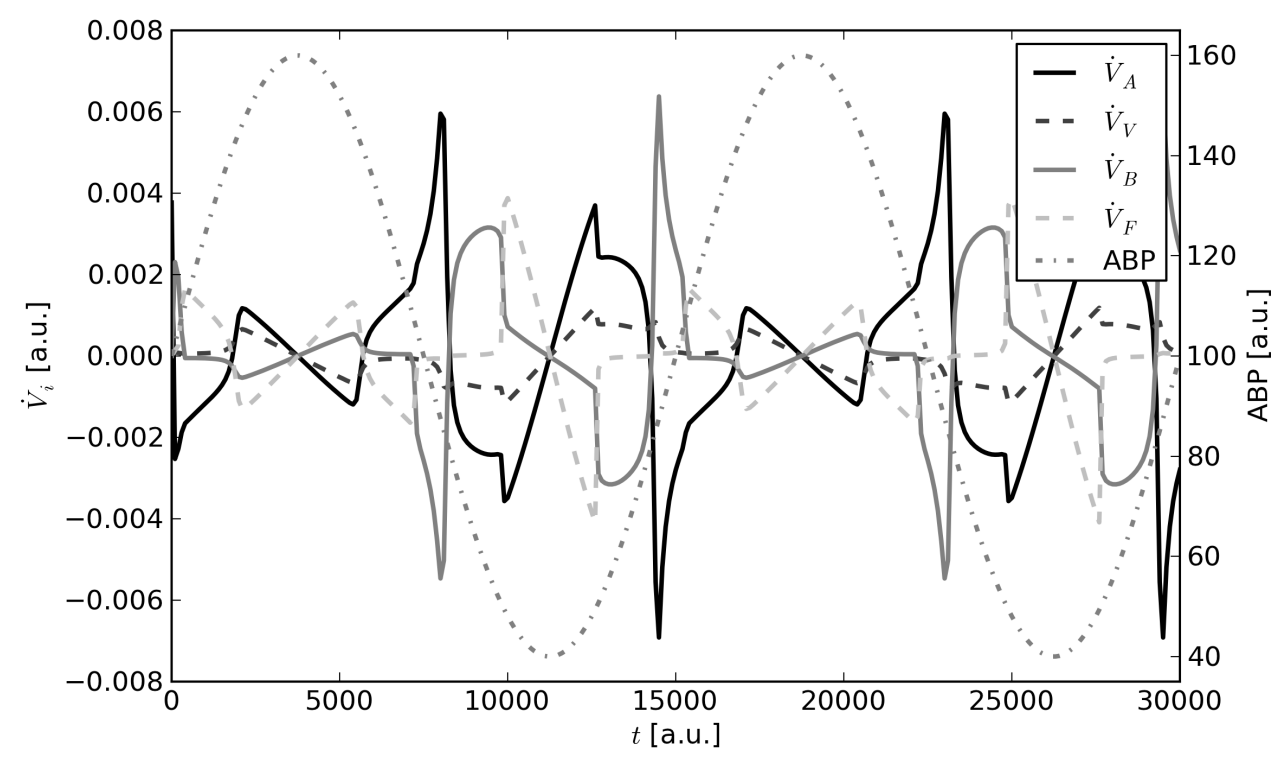

Figure 6.22: $\dot{V}_{i}$ against time with $\alpha=2.0$ and $V_{E}=30 \mathrm{ml}$ (sHOM; case 4$)$.

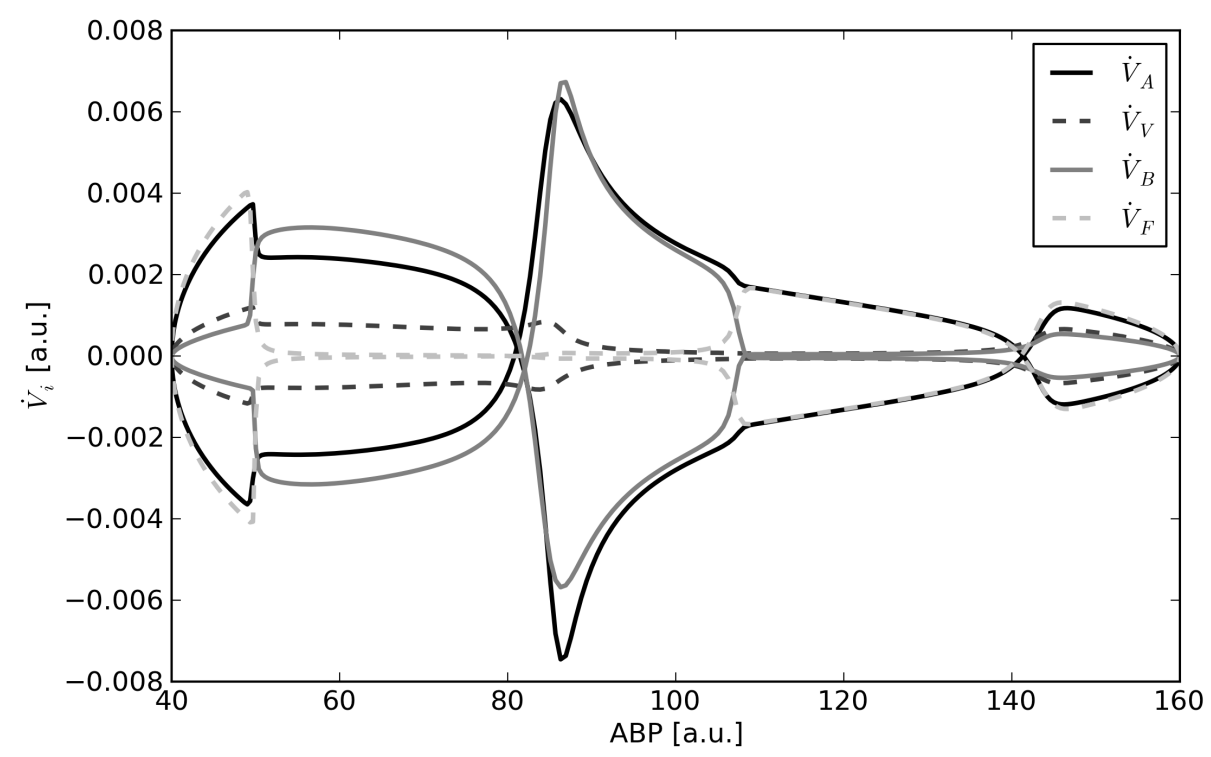

Figure 6.23: $\dot{V}_{i}$ against ABP with $\alpha=2.0$ and $V_{E}=30 \mathrm{ml}$ (sHOM; case 4). 


\subsection{Summary}

In this chapter, the differences between the bHOM and sHOM models have been examined. For the scenarios, in which the simulations were done without a swelling, the deviations between both models are relatively small. Therefore, the resulting CBFs are nearly identical. The biggest differences between both models lies in the different characteristics of the resistances of the veins and slightly increased volume changes of the veins. Also, the volume development in the sHOM model for the first case, is smoother than for the bHOM model.

But for the simulations, which included a swelling, the sHOM model differs a lot from the bHOM model for the time segment, when there is no CSF left to absorb. As for case 3, a drop in CBF for the sHOM model can be seen for ABP values above $100 \mathrm{mmHg}$. The cause for this difference lies in the increased volume change in the blood vessels system. As the CSF compartment cannot compensate this increase, the brain tissue gets more compressed which results in an increased ICP and an increased pressure inside the capillaries and veins. The pressure increase of the capillaries is then responsible for the drop in the CBF.

The same holds for case 4, but the reason is different. There, mainly the increase of the artery compartment inside the ABP range, in which the CSF compartment is depleted, is responsible for the increase in volume of the blood vessel system. This is due to the autoregulation mechanism, which is trying to compensate the drop in $\mathrm{CBF}$ for $\mathrm{ABP}$ values below $100 \mathrm{mmHg}$ by increasing the diameter of the arteries. But the CSF compartment is already depleted and therefore not able to compensate this volume change. The results is, that the brain tissue gets more compressed, which results in the exact behavior, seen in case 3 .

In the next chapter, in addition to the starling resistor, another alteration of the model will be incorporated. There, to better reflect the natural circumstances, the model will be altered to simulate the haemodynamics of both hemispheres of the human brain. 


\section{Chapter 7}

\section{General behavior of esHOM model}

The modification of the basic Haemodynamical and Oxygen transport Model (bHOM), by using a starling resistor, was the first step, in order to simulate the natural circumstances of the haemodynamics of the human brain better. In this chapter, another step is taken. Here, the blood flow is divided into two flows for each hemisphere of the human brain. As described in section 3.4.2, in both hemispheres the autoregulation is independent and both hemispheres contribute to the cerebrospinal fluid (CSF) production.

For the scenario, in which the autoregulation is identical in the sense, that at both hemispheres the same parameters are used, there should only be small differences between the sHOM and the esHOM model for the cases without a swelling. From a simulating point of view, the more interesting scenarios are, when a swelling occurs and the autoregulation differs between both hemispheres. As this can happen in real life, with this extension it is possible to simulate the case, in which the autoregulation is impaired in one hemisphere and works as intended in the other.

Therefore, in the next sections, the differences between the sHOM and esHOM models are shown for the corresponding simulations. This will be done for the same type of simulations as in the previous chapters, with an autoregulation mechanism in the same state in each hemisphere. Afterwards, the coupling of each hemisphere with the other is examined, in case of different states of the autoregulation in each hemisphere.

As the extension of the sHOM model is implemented in a symmetrical way, the values for pressures, compliances, resistances and volume developments of and inside each corresponding compartment are identical, as long as the same set of parameters is used for each hemisphere. Therefore, for simplicity and clarity, in the following only the graphs for one hemisphere are shown. For the last cases in this chapter, in which a different $\alpha$ for each hemisphere is used, the values of each hemisphere will be shown separately. 


\subsection{Introductory remark}

In order to compare the sHOM model with the esHOM model, the parameters of the autoregulation mechanism have to be altered, according to chapter 4 . In addition, the parameter $\tau$ has to be altered, too.

As already seen in the previous chapter, due to the starling resistor, a splitting in some of the graphs occurs. This splitting can be reduced with a smaller default value for $\tau$. But as the effect only occurs for the last case (compare section 6.2.2), the value for $\tau$ was not altered.

In the esHOM model, the splitting gets worse and even results in some non-realistic values for the cerebral blood flow $(\mathrm{CBF})$ in case 4 . In order to compare the esHOM model with the sHOM model, a different value for $\tau$ was chosen, namely $\tau=2.5 \mathrm{~s}$. With this value, the non-realistic results vanish and the splittings, seen in some graphs, have a comparable magnitude.

This alteration can be justified, as the primary goal of this chapter is to examine, how the splitting into two hemispheres alters the CBF, when compared to the sHOM model, and the coupling between both hemisphere for a different autoregulation in both hemispheres.

\subsection{No Swelling}

In this section, the behavior of the esHOM model is examined for those scenarios, in which the autoregulation in both hemispheres is identical $\left(\alpha^{L}=\right.$ $\left.\alpha^{R}\right)$ and no swelling occurs $\left(V_{E}=0 \mathrm{ml}\right)$. In case 1 , the autoregulation is disabled in general $\left(\alpha^{i}=0\right)$, whereas in case 2 , the autoregulation is enabled in both hemispheres $\left(\alpha^{i}=1.4\right)$.

\subsubsection{Case 1: no swelling, no autoregulation}

In figure 7.1 the $\mathrm{CBF}$ of the sHOM model and the $\mathrm{CBF}^{*}$ of one hemisphere of the esHOM model is plotted against the arterial blood pressure (ABP), whereas both CBFs are plotted against different $y$-axes. Both graphs have an identical slope, whereas the CBF of the sHOM model is twice as big as for one hemisphere in the esHOM model. As the whole flux through the brain is the sum of the fluxes through each hemisphere,

$$
\mathrm{CBF}=\mathrm{CBF}^{L}+\mathrm{CBF}^{R}
$$

the blood flow in both models can be considered equal. This suggests, that there will be no big differences for this case between both models. For example, the pressures inside each compartment are identical within the computational accuracy.

\footnotetext{
${ }^{*}$ Here, CBF does not mean CBF from a medical point of view, but from a model point of view. In case of the esHOM model, this means the flow through each hemisphere.
} 


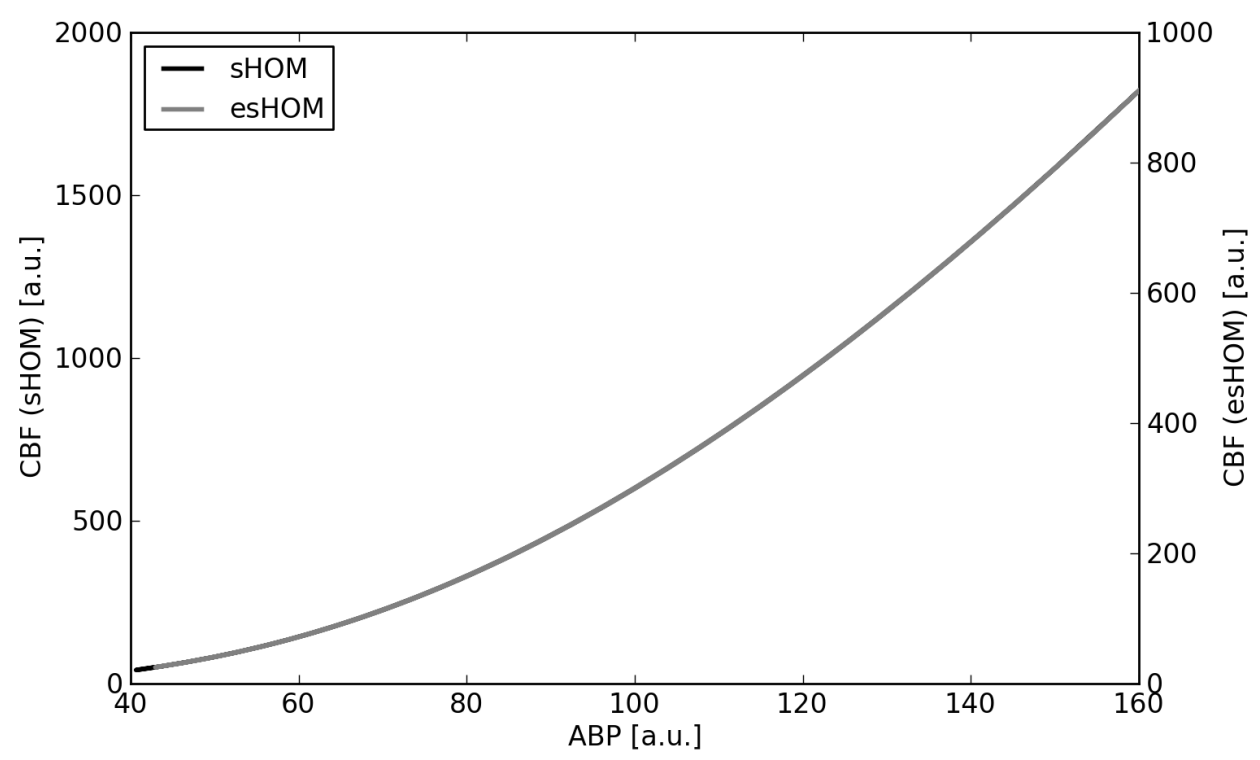

Figure 7.1: $\mathrm{CBF}$ against $\mathrm{ABP}$ with $\alpha^{(i)}=0$ and $V_{E}=0 \mathrm{ml}$ ( $\mathrm{sHOM}$ and esHOM; case 1)

Therefore, in the rest of this chapter, only those parts of the model are discussed, where there are differences between the two models.

\section{Resistance}

From the identical pressures it can already be deduced, that $R_{A C}^{i}$ of each hemisphere has to be two times $R_{A C}$ for all $\mathrm{ABP}$ values. For the resistance of the veins in each hemisphere, the same holds, namely: $R_{V S}^{i}$ is two times bigger than $R_{V S}$ for all $\mathrm{ABP}$ values, as deduced for the standard values for the resistances in section 4.4.1. The default values of the resistance of the arteries and veins of both models are summarized in table 7.1, as are their minimal and maximal values.

\section{Compliance}

The compliances for the brain and the veins are identical to the sHOM model, which can be seen in table 6.2 (sHOM) and 7.2 (esHOM). The reason for this is quite simple: As the pressures are identical for this case in both models, so has to be the compliance of the brain, as this part of the model has not changed. The same is true for the veins: Although there are two separated compartments for the veins, the implementation of the compliance 


\begin{tabular}{l|r|r|r|r} 
Resistance $\left[\frac{\mathrm{mmHg}}{\mathrm{ml} / \mathrm{min}}\right]$ & $\min$ & default & $\max$ & range \\
\hline$R_{A C}$ (single) & 0.052 & 0.125 & 0.849 & 0.797 \\
$R_{A C}^{i}$ (dual) & 0.104 & 0.250 & 1.698 & 1.594 \\
$R_{V S}$ (single) & 0.0167 & 0.0167 & 0.0202 & 0.0035 \\
$R_{V S}^{i}$ (dual) & 0.0334 & 0.0334 & 0.0403 & 0.0069
\end{tabular}

Table 7.1: Resistance ranges with $\alpha^{i}=0$ and $V_{E}=30 \mathrm{ml}$ (sHOM and esHOM; case 1 ). Values for $R_{X Y}^{i}$ are two times higher than the corresponding values for $R_{X Y}$.

has not changed. As the pressure inside each compartment of the veins is the same as in the sHOM model, so is their compliance.

\begin{tabular}{l|r|r|r|r} 
Compliance $\left[\frac{\mathrm{ml}}{\mathrm{mmHg}}\right]$ & $\min$ & default & $\max$ & range \\
\hline$C_{A B}^{i}$ & 0.106 & 0.106 & 0.106 & 0.00 \\
$C_{V B}^{i}$ & 0.189 & 0.444 & 1.178 & 0.989 \\
$C_{B}$ & 0.182 & 0.350 & 0.595 & 0.413
\end{tabular}

Table 7.2: Ranges for the compliances with $\alpha^{i}=0$ and $V_{E}=30 \mathrm{ml}$ (esHOM; case 1$)$.

The compliance of the arteries is constant in both models, due to the disabled autoregulation. Their values differ by a factor $\sqrt{2}$, which stems from the deduced value in section 4.4.1 as the new default value.

In the following, these kind of differences, like that for the resistances and compliances, will also be ignored, as they do not contribute to a better understanding of the underlying mechanisms, that drives the haemodynamic processes of the human brain.

\section{Volume}

Due to the similarities between the sHOM and esHOM model, the volume developments of the compartments, which are depicted in fig. 7.2, can easily be explained. As the pressures have the same development in both models and their compliances are modeled the same, the graphs for $\Delta V_{B}$ and $\Delta V_{V}^{i}$ are identical compared to the sHOM model.

The difference between both models is, that for one, in the extended model, the volume change of the complete venous blood vessel system is twice as big, when compared to the sHOM model, due to the same volume development of each venous system in both hemispheres. This stems from the design of the esHOM model, for which the motivation was to alter as much as is necessary, in order to be able to compare the sHOM directly with the esHOM model and reuse everything else. 


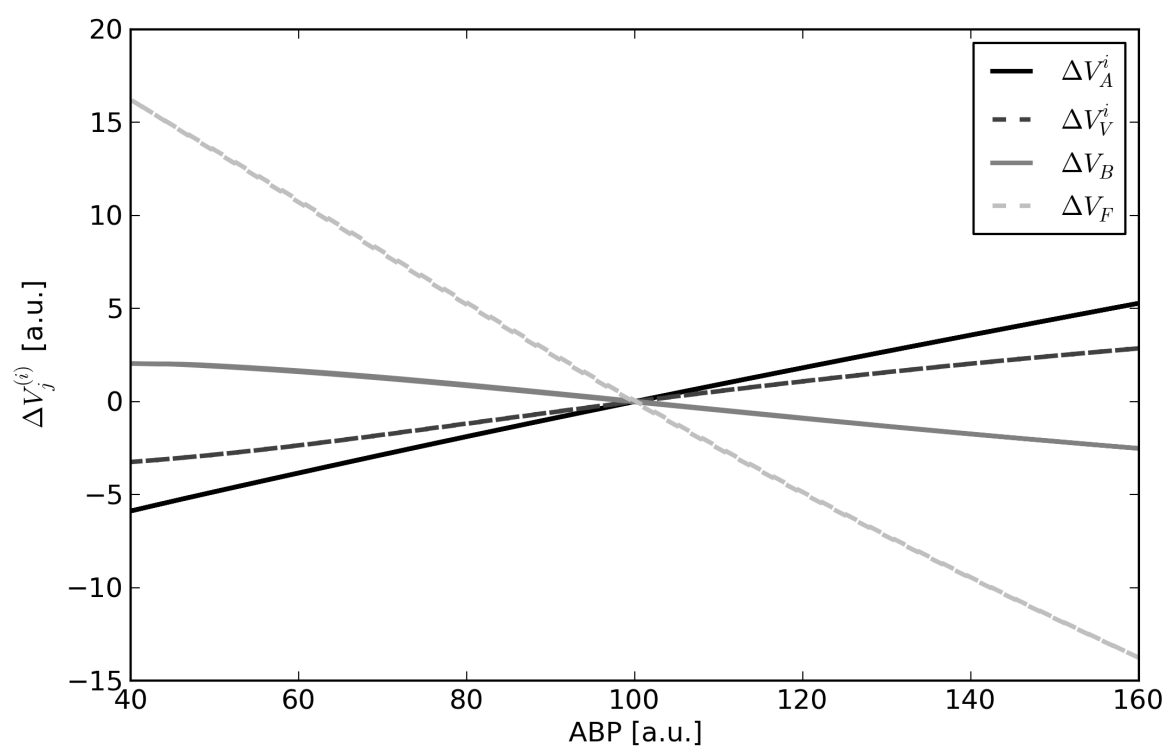

Figure 7.2: $\Delta V_{j}^{(i)}$ against $\mathrm{ABP}$ with $\alpha^{i}=0$ and $V_{E}=0 \mathrm{ml}$ (esHOM; case $1)$.

The course of the volume change of the arteries can be considered similar in both models. The change in volume for the arteries is smaller in each hemisphere, when compared to the sHOM model, but the total absolute change of the artery system is about $40 \%$ bigger in the extended model.

As before, the resulting volume difference is compensated by the CSF compartment. Due to the bigger volume change of the arteries and veins, here, the change in CSF volume is higher when compared to the sHOM model.

In table 7.3 the important values for the volume changes of each compartment are summarized.

\begin{tabular}{l|r|r|r}
$\Delta$ Volume $[\mathrm{ml}]$ & $\min$ & $\max$ & range \\
\hline$\Delta V_{A}^{i}$ & -5.88 & 5.28 & 11.16 \\
$\Delta V_{B}$ & -2.52 & 2.04 & 4.56 \\
$\Delta V_{F}$ & -13.77 & 16.22 & 29.99 \\
$\Delta V_{V}^{i}$ & -3.25 & 2.86 & 6.11
\end{tabular}

Table 7.3: Ranges for the volume changes for $\alpha^{i}=0$ and $V_{E}=0 \mathrm{ml}$ (esHOM; case 1$)$. 


\subsubsection{Case 2: no swelling, autoregulation}

Similarly to the previous section, there are no significant differences between both models for the case, in which there is no swelling $\left(V_{E}=0 \mathrm{ml}\right)$ and the autoregulation is enabled $\left(\alpha^{i}=1.4\right)$. The CBF through one hemisphere in the esHOM model is twice as small as in the sHOM model, due to the splitting of the flux through both hemispheres (compare figure 7.3).

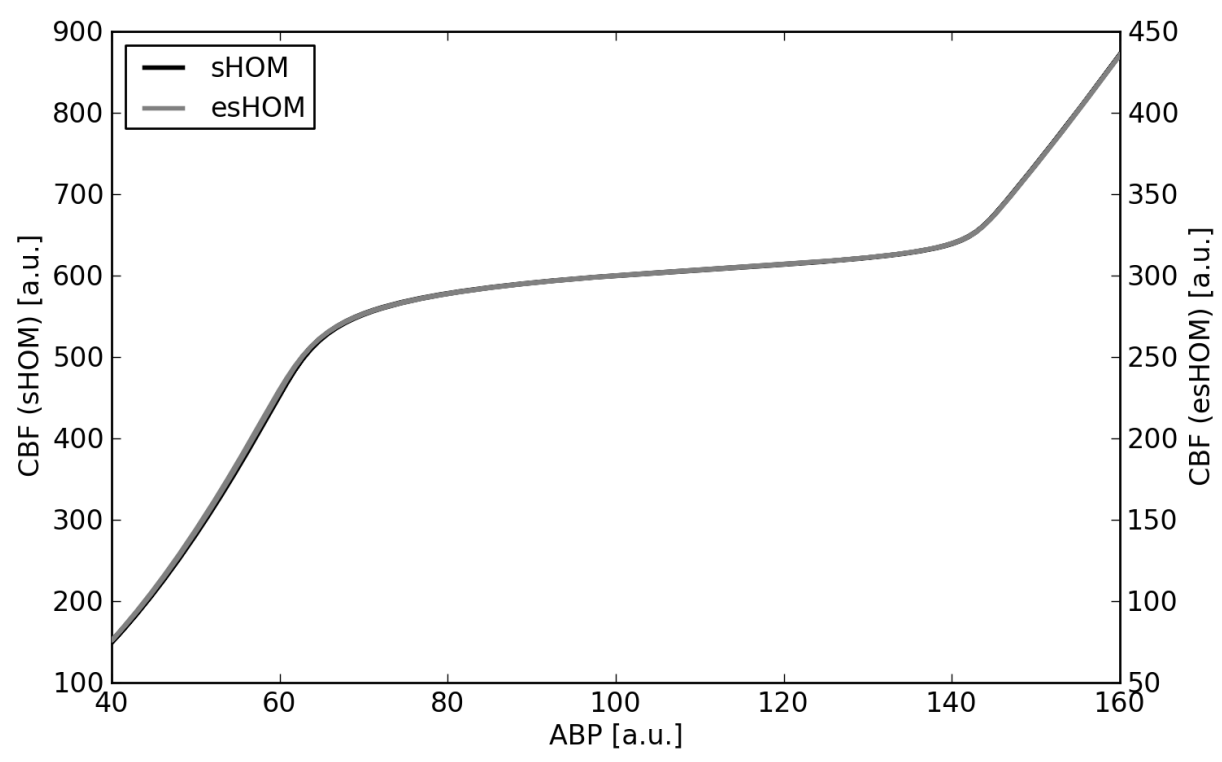

Figure 7.3: CBF against ABP with $\alpha=2(\mathrm{sHOM}), \alpha^{i}=1.4(\mathrm{esHOM})$ and $V_{E}=0 \mathrm{ml}$ (sHOM and esHOM; case 2).

Therefore, the development of the compliances, resistances and pressures can be deduced from the corresponding curves of the sHOM model and from the knowledge gained in the previous section. The differences between the expected and calculated values are in the range of one-tenth percent and can be neglected.

The same holds for the development of the volumes of each compartment, whereas before, the remaining difference in volume development is compensated by the CSF compartment. The corresponding graphs for the volume development are shown in figure 7.4.

\subsection{Swelling}

In this section, the simulations of the previous section are repeated with the difference, that a swelling occurs $\left(V_{E}=30 \mathrm{ml}\right)$. As before in case 3, 


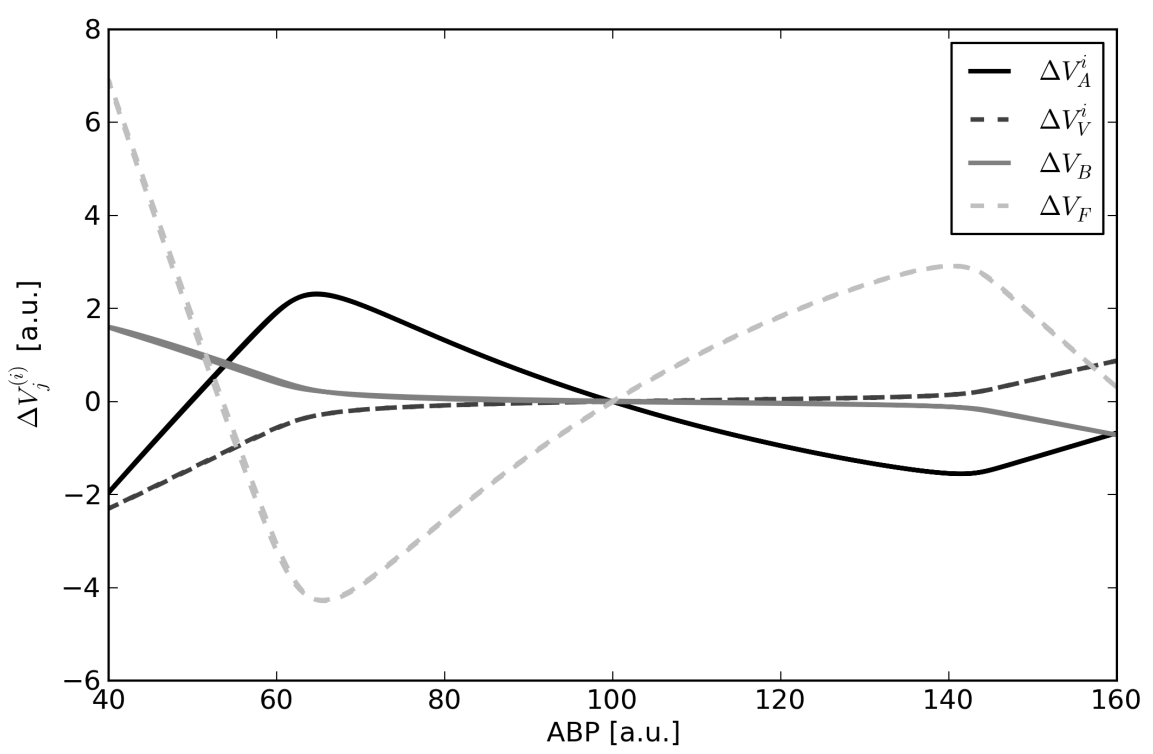

Figure 7.4: $\Delta V_{j}^{(i)}$ against ABP with $\alpha^{i}=1.4$ and $V_{E}=0 \mathrm{ml}$ (esHOM; case $2)$.

the autoregulation is disabled in general $\left(\alpha^{i}=0\right)$, whereas in case 4 , the autoregulation is enabled in both hemispheres $\left(\alpha^{i}=1.4\right)$. Furthermore, $\Delta V_{F}=V_{F}$ and not $V_{F}-V_{F n}$ is plotted, due to the swelling.

\subsubsection{Case 3: swelling, no autoregulation}

From the previous simulations for the bHOM and sHOM models, the CBF for the esHOM model can be deduced for the case, in which the autoregulation is disabled and a swelling is simulated. The effect of the starling resistor on the $\mathrm{CBF}$, which resulted in a decrease of $\mathrm{CBF}$ for the segment, where the CSF compartment was depleted, should be bigger.

Similar to case 3 for the sHOM model, this segment should be starting around a raising ABP of approx. $100 \mathrm{mmHg}$. As the increase of volumes of the total artery and vein compartments is bigger (compare case 1), this should result in a bigger increase of the intracranial pressure (ICP) than for the ICP in the corresponding simulations with the sHOM model. Therefore, a bigger increase in pressures in the other compartments is expected than in case 3 for the sHOM model.

This is exactly, what can be seen in figure 7.5, which shows the CBF of the sHOM and esHOM model for disabled autoregulation and a swelling. Here, for an ABP above approx. $100 \mathrm{mmHg}$, the drop in $\mathrm{CBF}$ in each hemi- 
sphere for the esHOM model is quite obvious.

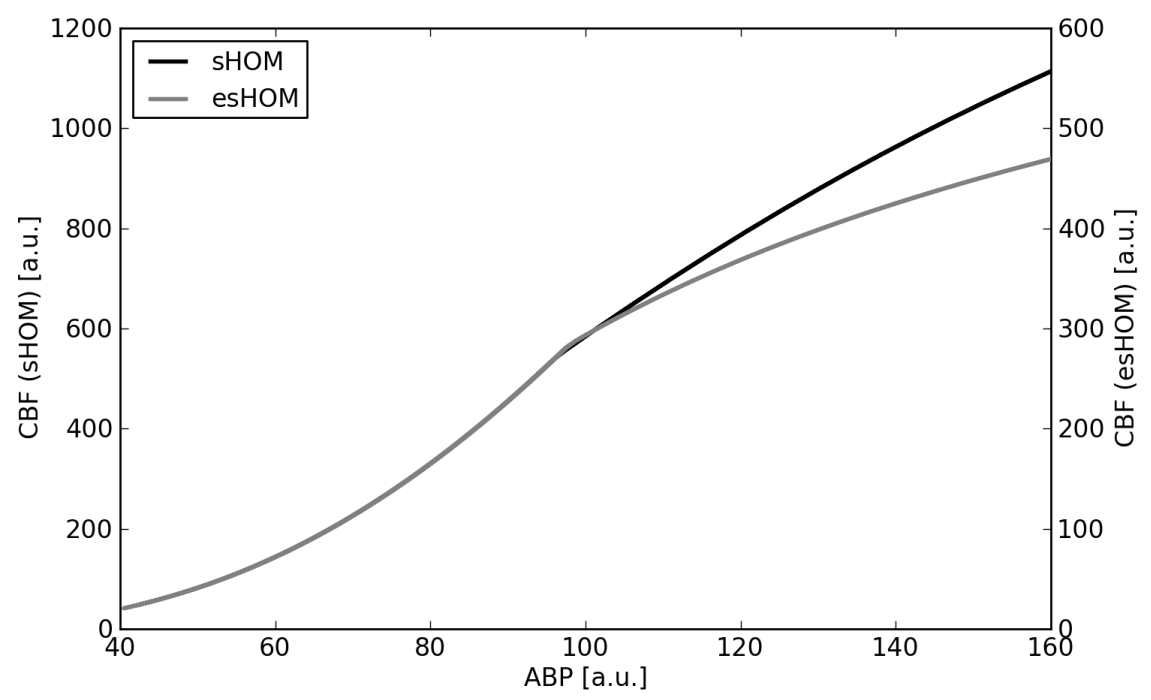

Figure 7.5: $\mathrm{CBF}$ against $\mathrm{ABP}$ with $\alpha^{i}=0$ and $V_{E}=30 \mathrm{ml}$ (sHOM and esHOM; case 3 ).

From the CBF, it can be deduced, that there will only be small differences between both models from a qualitative point of view, although the extremal values of some properties should be significantly higher in the esHOM model, when compared to their corresponding values in the sHOM model.

\section{Pressure}

For ABP values below approx. $95 \mathrm{mmHg}$ there is no difference between both models. Above $95 \mathrm{mmHg}$, the pressure inside the blood vessels and brain tissue increases more rapidly in the esHOM model, but the slope of the pressure curves has a similar run. The extremal points are summarized in table 7.4.

\begin{tabular}{l|r|r|r|r} 
Pressure $[\mathrm{mmHg}]$ & min & default & $\max$ & range \\
\hline$p_{A}^{i}$ & 40.00 & 100.00 & 160.00 & 120.00 \\
$p_{C}^{i}$ & 6.45 & 25.00 & 76.67 & 71.22 \\
$p_{V}^{i}$ & 5.80 & 15.00 & 61.93 & 56.13 \\
$p_{B}$ & 5.47 & 10.00 & 53.21 & 47.74
\end{tabular}

Table 7.4: Pressure ranges for $\alpha^{i}=0$ and $V_{E}=30 \mathrm{ml}$ (esHOM; case 3).

Compared to the pressure values of the sHOM model (see table 6.6), 
the pressure increase at an $\mathrm{ABP}$ of $160 \mathrm{mmHg}$ inside the capillaries is slightly smaller (around $4 \mathrm{mmHg}$ ) than for the veins and brain tissue (around $7 \mathrm{mmHg})$.

\section{Resistance}

As for the resistances, for ABP values below $95 \mathrm{mmHg}$, the same development occurs as in previous simulation of the esHOM model with disabled autoregulation and no swelling.

Above an $\mathrm{ABP}$ of $95 \mathrm{mmHg}$, the resistance of the veins increases almost linearly, but with a slightly higher slope than in the corresponding simulation with the sHOM model. The decrease of the resistance of the arteries for a increasing volume of the arteries is a little bit smaller, when compared to the sHOM model. The extremal points for the resistances are summarized in table 7.5 for the sHOM and esHOM model. To compare the values between both models, the values for the esHOM model have to be multiplied by two, as derived in section 4.4.1.

\begin{tabular}{l|r|r|r} 
Resistance $\left[\frac{\mathrm{mmHg}}{\mathrm{ml} / \mathrm{min}}\right]$ & $\min (\mathrm{ABP})$ & default & $\max (\mathrm{ABP})$ \\
\hline$R_{A C}$ (single) & 0.8490 & 0.125 & 0.0776 \\
$R_{A C}^{i}$ (dual) & 1.1698 & 0.250 & 0.178 \\
$R_{V S}$ (single) & 0.0202 & 0.0167 & 0.0450 \\
$R_{V S}^{i}$ (dual) & 0.0403 & 0.0334 & 0.1195
\end{tabular}

Table 7.5: Resistance ranges for $\alpha^{i}=0$ and $V_{E}=30 \mathrm{ml}$ (sHOM and esHOM; case 3$)$.

Responsible for the difference between both models concerning the resistances is a difference in the development of the corresponding compliances, due to the different pressures.

\section{Compliances}

The development of the compliances is shown in figure 7.6. As before, for $\mathrm{ABP}$ values below $95 \mathrm{mmHg}$, there is no difference between the simulations with and without a swelling for disabled autoregulation.

Due to the bigger increase of ICP for increasing ABP values above $95 \mathrm{mmHg}$, the compliance of the brain tissue decreases more rapidly, when compared to the simulation with the sHOM model (compare table 6.7 and 7.6). But the slope of both models is still identical from a qualitative point of view.

As for the compliance of the veins, there is a slight bend around an ABP of $95 \mathrm{mmHg}$, which is hard to see in case 3 of the sHOM model. From there on, the decrease is slightly slower, compared to the simulations with the 


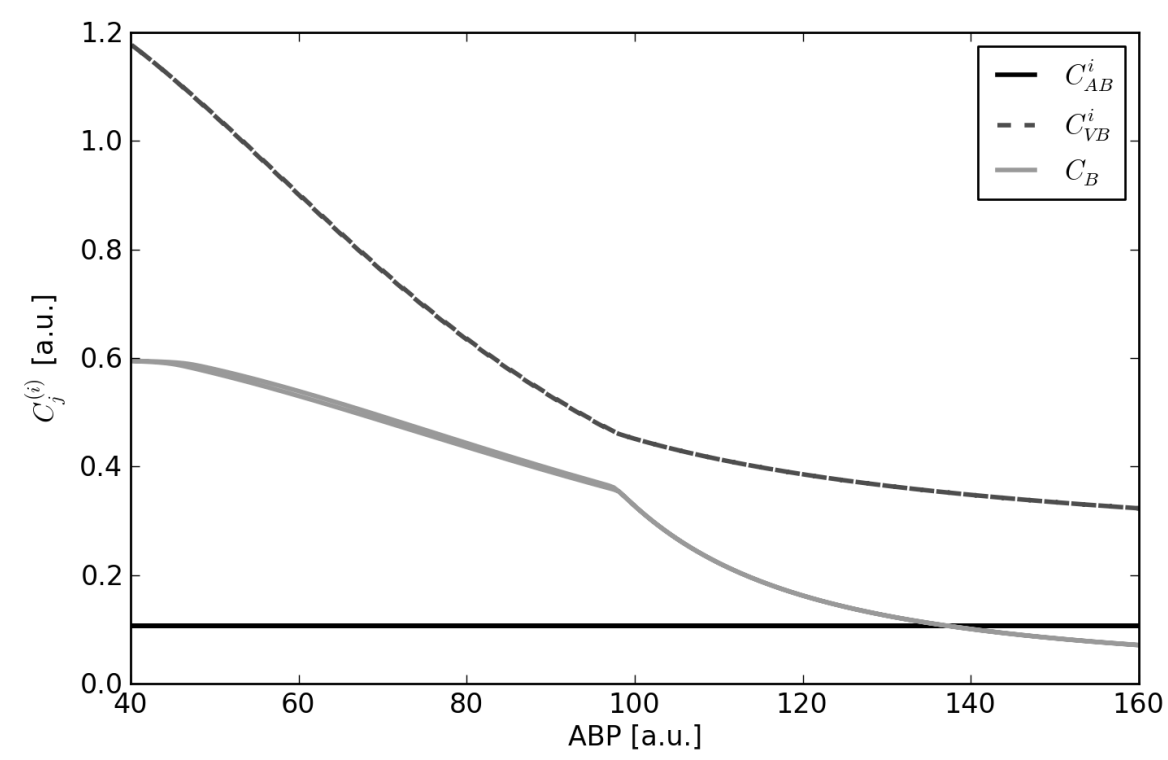

Figure 7.6: $C_{j}^{(i)}$ against $\mathrm{ABP}$ with $\alpha^{i}=0$ and $V_{E}=30 \mathrm{ml}$ (esHOM; case 3).

\begin{tabular}{l|r|r|r|r} 
Compliance $\left[\frac{\mathrm{ml}}{\mathrm{mmHg}}\right]$ & $\min$ & default & $\max$ & range \\
\hline$C_{A B}^{i}$ & 0.106 & 0.106 & 0.106 & 0.00 \\
$C_{V B}^{i}$ & 0.032 & 0.444 & 1.178 & 1.146 \\
$C_{B}$ & 0.071 & 0.350 & 0.595 & 0.524
\end{tabular}

Table 7.6: Ranges for the compliances for $\alpha^{i}=0$ and $V_{E}=30 \mathrm{ml}$ (esHOM; case 3$)$.

sHOM model. From this behavior can be deduced, that the development of the ICP and pressure inside the veins has a more similar slope here, than in the sHOM model, as the compliance of the veins mainly depends on the pressure difference between both.

\section{Volume}

Figure 7.7 shows the volume development of each compartment, which contains no surprises, when compared to the results of the previous simulations with the esHOM model without a swelling and the sHOM model with a swelling.

For $\mathrm{ABP}$ values below $95 \mathrm{mmHg}$, the results for simulations with the sHOM and esHOM model can be more or less considered equal. Above this 


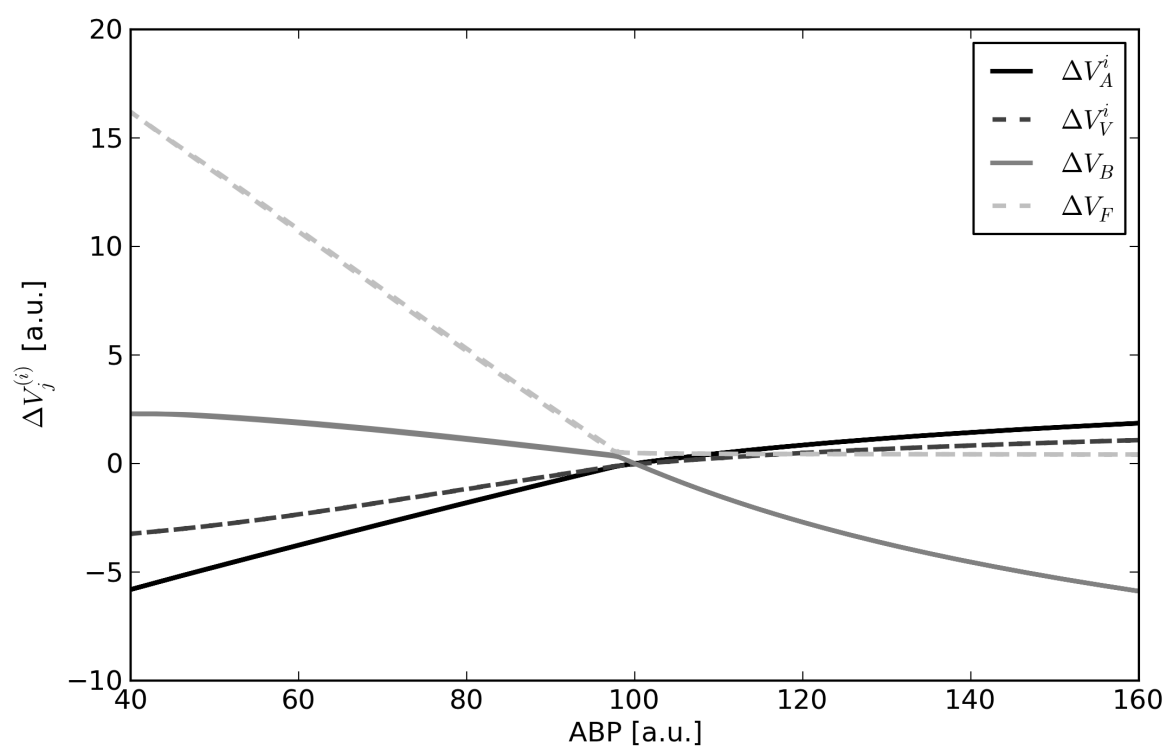

Figure 7.7: $\Delta V_{j}^{(i)}$ against $\mathrm{ABP}$ with $\alpha^{i}=0$ and $V_{E}=30 \mathrm{ml}$ (esHOM; case $3)$.

value, the volume of the brain tissue decreases a little bit faster than for the corresponding simulation with the sHOM model. This can be seen in table 7.7, which summarizes the extremal values for the minima and maxima, and table 6.8. Responsible for this development is the bigger increase of the total volume of all blood vessels, which cannot be compensated by the depleted CSF compartment.

\begin{tabular}{l|r|r|r}
$\Delta$ Volume $[\mathrm{ml}]$ & $\min$ & $\max$ & range \\
\hline$\Delta V_{A}^{i}$ & -5.80 & 1.86 & 7.66 \\
$\Delta V_{B}$ & -5.88 & 2.30 & 8.18 \\
$\Delta V_{F}$ & 0.42 & 16.19 & 16.61 \\
$\Delta V_{V}^{i}$ & -3.24 & 1.08 & 4.32
\end{tabular}

Table 7.7: Ranges for the volume changes with $\alpha^{i}=0$ and $V_{E}=30 \mathrm{ml}$ (esHOM; case 3).

As for the small differences between the corresponding simulations with and without a swelling for an ABP of $40 \mathrm{mmHg}$ : These stem from numerical inaccuracies, when calculating the corresponding values of the simulation. This can be deduced from the fact, that there are no significant differences between the simulated values for the pressures, resistances and compliances 
between both the simulations with and without a swelling.

\subsubsection{Case 4: swelling, autoregulation}

As for the last case with identical autoregulation mechanism in both hemispheres, in which the simulations are done with enabled autoregulation and a swelling: The course of the CBF differs from the sHOM model inside the interval, in which the CSF compartment is depleted, as it was the case in the previous section, in which the autoregulation was disabled. For ABP values outside this interval, the $\mathrm{CBF}$ of the sHOM model and esHOM model can be considered identical, which can be seen in figure 7.8.

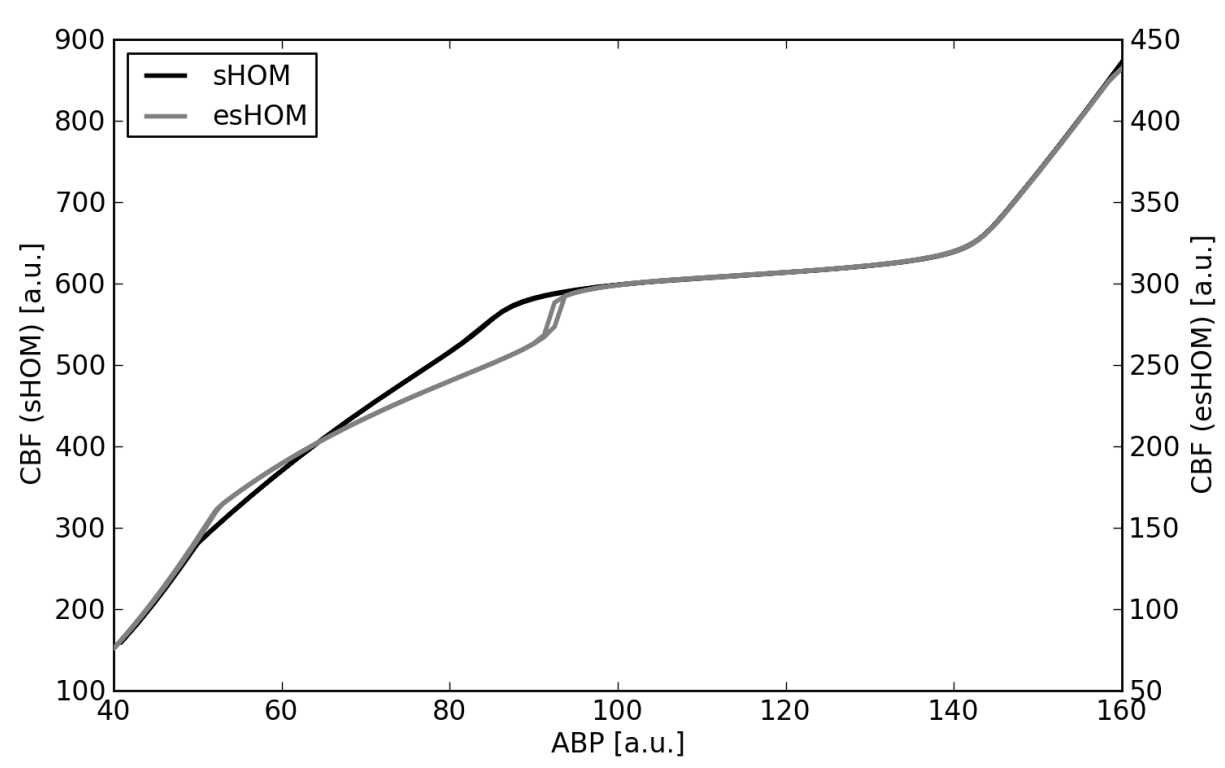

Figure 7.8: $\mathrm{CBF}$ against ABP with $\alpha=2(\mathrm{sHOM}), \alpha^{i}=1.4(\mathrm{esHOM})$ and $V_{E}=30 \mathrm{ml}$ (case 4$)$.

But between an $\mathrm{ABP}$ of around 50 and $95 \mathrm{mmHg}$, the behavior differs. For decreasing $\mathrm{ABP}$ at $95 \mathrm{mmHg}$, there is a sharp drop in $\mathrm{CBF}$ for the esHOM model. From there on, the CBF decreases with a smaller slope than the $\mathrm{CBF}$ of the sHOM model. At an ABP around $52 \mathrm{mmHg}$, there is another sharp bend. The corresponding bend of the sHOM model appears at a smaller $\mathrm{ABP}$ of around $50 \mathrm{mmHg}$, from where on both have a similar slope for decreasing ABP.

The sharp drop of the $\mathrm{CBF}$ at $95 \mathrm{mmHg} \mathrm{ABP}$ in the esHOM model also appears in an analog way in the sHOM model. But there, the interval, in which this drop takes place, is broader and not as steep, and also happens 
for smaller $\mathrm{ABP}$ values at around 82 to $86 \mathrm{mmHg}$.

\section{Pressure}

The reason for the sharp drop in CBF, seen in fig. 7.8, is the step increase of pressure inside all compartments at a decreasing ABP of approx. $95 \mathrm{mmHg}$, as can be seen in fig. 7.9.

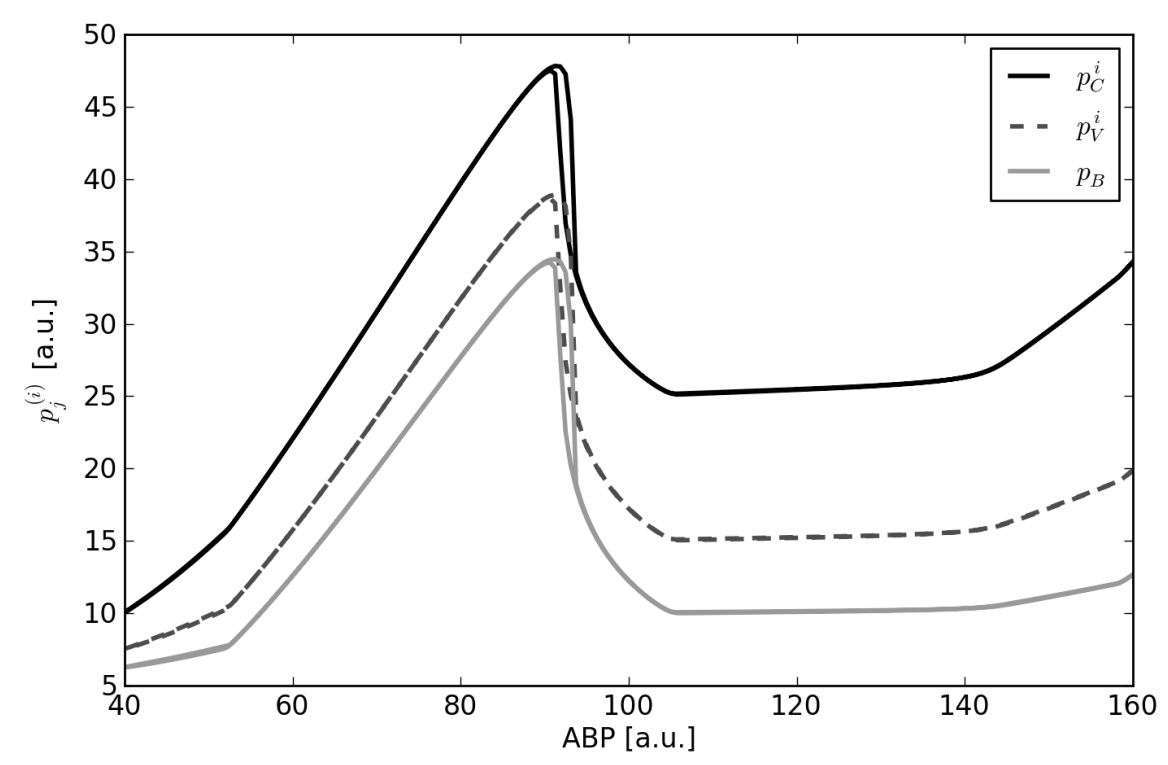

Figure 7.9: $p_{j}^{(i)}$ against ABP with $\alpha^{i}=1.4$ and $V_{E}=30 \mathrm{ml}$ (esHOM; case $4)$.

When compared to the local maxima of pressure values for the $\mathrm{SHOM}$ model, here, the pressure reaches values, that are approx. $8 \mathrm{mmHg}$ higher. Also, the increase for decreasing $\mathrm{ABP}$ values takes place inside a much shorter ABP interval.

There is another difference between both simulations. For increasing ABP values above $156 \mathrm{mmHg}$, there is a small increase of the pressure inside each compartment, when compared to the sHOM case. As will be seen later, here, due to the increased volume of the blood vessels, the CSF compartment is depleted, again.

\section{Resistance}

The graphs for the resistances are shown figure 7.10. As for the resistance of the veins, the maximum value has moved from an $\mathrm{ABP}$ of around $80 \mathrm{mmHg}$ in the sHOM model to an ABP of $90 \mathrm{mmHg}$. Similar to the development 
of the pressures, at an $\mathrm{ABP}$ of approx. $90 \mathrm{mmHg}$, there is a sharp drop for increasing ABP.

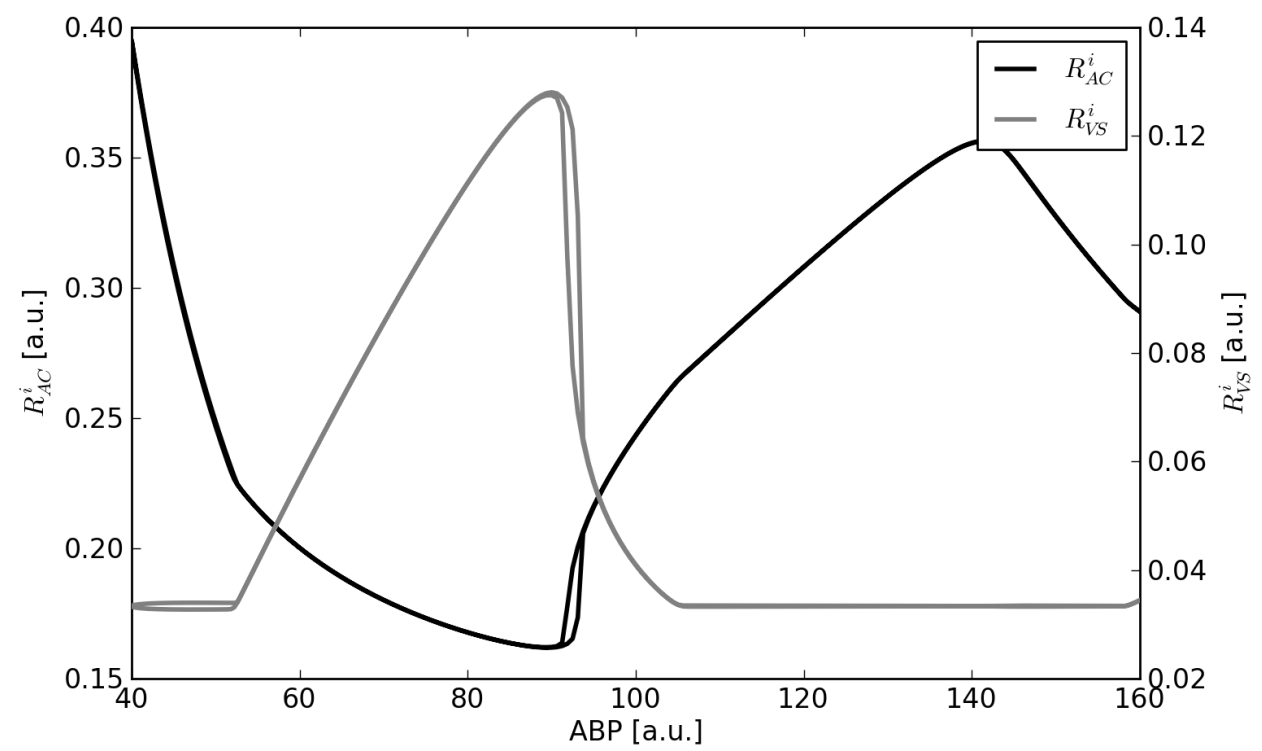

Figure 7.10: $R_{X Y}^{i}$ against $\mathrm{ABP}$ with $\alpha^{i}=1.4$ and $V_{E}=30 \mathrm{ml}$ (esHOM; case 4$)$.

The same can be seen in an analog way for the resistance of the arteries. Here, the minimal value of $R_{A C}^{i}$ has moved towards a higher ABP value, from which onwards there is a sharp increase with increasing ABP. For higher ABP values afterwards this sharp increase, the course is similar to the development of the resistances in the sHOM model.

At the upper end of the ABP range, $R_{V S}^{i}$ increases, whereas $R_{A C}^{i}$ decreases, due to the aforementioned depletion of the CSF compartment.

\section{Compliance}

Not surprisingly, the jump seen in CBF can also be seen in all graphs for the compliances (compare figure 7.11).

From the compliance of the arteries $C_{A B}^{i}$, it can be seen, that the extremal autoregulation point for an $\mathrm{ABP}$ below $100 \mathrm{mmHg}$ is reached at a higher $\mathrm{ABP}$ value, than for the sHOM model. Also, the increase in $C_{A B}^{i}$ for decreasing $\mathrm{ABP}$ values below $100 \mathrm{mmHg}$ happens in a shorter interval. The maximal value of $C_{A B}^{i}$ is already reached for an ABP of approx. $90 \mathrm{mmHg}$, whereas for the sHOM model, this point was reached not until an ABP of around $60 \mathrm{mmHg}$. This behavior of the autoregulation is responsible for 


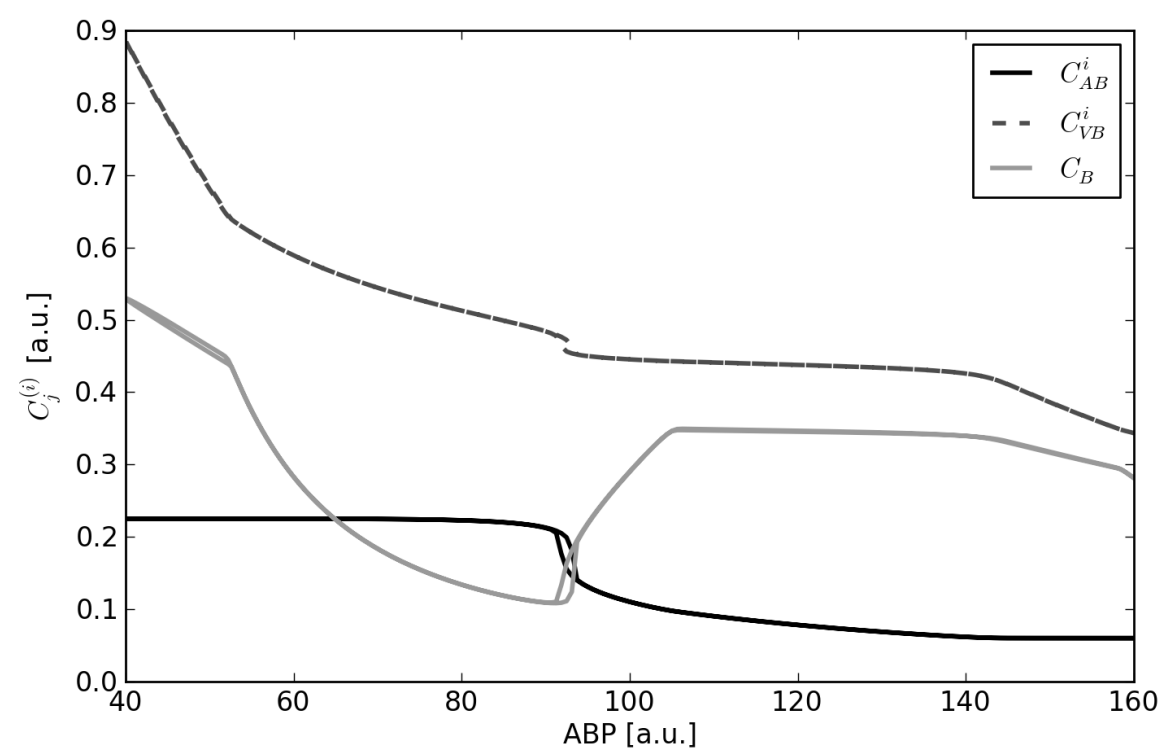

Figure 7.11: $C_{j}^{(i)}$ against $\mathrm{ABP}$ with $\alpha^{i}=1.4$ and $V_{E}=30 \mathrm{ml}$ (esHOM; case $4)$.

the different behavior of the esHOM model, when compared to the sHOM model. As the maximal value for $C_{A B}^{i}$ is reached for a higher $\mathrm{ABP}$ value, all local extremal points of the pressures and resistances are also occurring at this $\mathrm{ABP}$ value.

The reason for this can easily be seen in the development of the volumes of each compartment, which will be examined in the next section.

\section{Volume}

In figure 7.12, the difference of the behavior of the previous graphs compared to the sHOM model can easily be explained: For an ABP below $100 \mathrm{mmHg}$, the arteries try to compensate the drop in $\mathrm{CBF}$, due to the decrease in $\mathrm{ABP}$, by increasing the diameter of the arteries.

The difference between the sHOM and esHOM model lies in the fact, that, to obtain the same effect for stabilizing the CBF, the dilation of the arteries must be bigger in order to reduce their resistance (compare volume development in sections 7.2.2 and 6.1.2). As the CSF compartment is already completely depleted, this volume change results in an increase of pressure inside all compartments. To compensate this increase in pressure, which would result in a decrease of $\mathrm{CBF}$, the diameter of the arteries has to be increased further. This results in the steep increase in volume of the arteries 


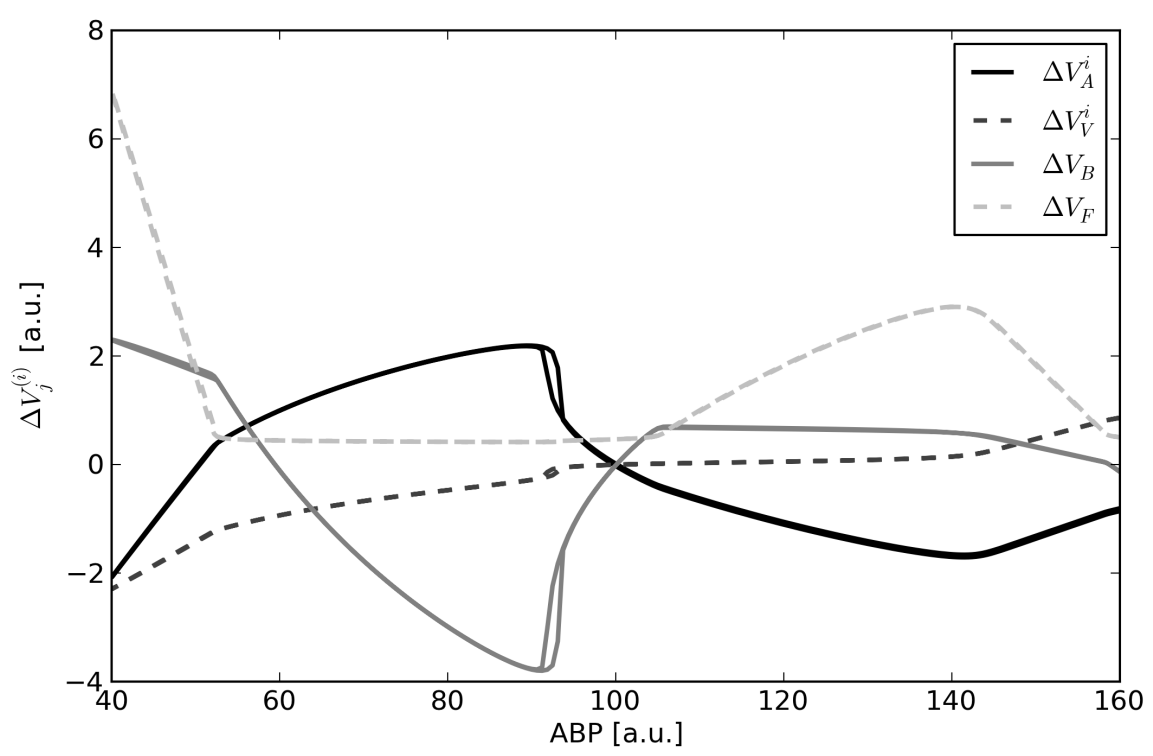

Figure 7.12: $\Delta V_{j}^{(i)}$ against $\mathrm{ABP}$ with $\alpha^{i}=1.4$ and $V_{E}=30 \mathrm{ml}$ (esHOM; case 4$)$.

for decreasing ABP. As the active dilation of the arteries is limited, the maximum value is reached at a higher ABP.

Due to this different behavior of the arteries, the brain tissue is more compressed than for the corresponding simulations with the sHOM model, which results in a higher ICP. As the ICP increases faster than the pressure inside the veins, the veins are more compressed, which can be seen in the graphs for the volume development.

\subsection{Mixed autoregulation}

As mentioned earlier, the autoregulation is modeled independently in each hemisphere. Therefore, it is possible to simulate the situation, in which the autoregulation is impaired in one hemisphere while still working in the other, which actually can happen in real life. This will be done, as before, for the cases, in which there is no swelling and a swelling is simulated.

The model allows to simulate this state and examine, how the haemodynamical processes in each hemispheres are coupled. For the following simulations, the autoregulation was disabled in the left $\left(\alpha^{L}=0\right)$ and enabled in the right hemisphere $\left(\alpha^{R}=1.4\right)$. For simplicity, here, only the CBFs, the pressures and volume developments of each hemisphere is examined, as from these in principal the course of the resistances and compliances 
can be deduced.

The graphs for the simulations with mixed and identical autoregulation are depicted in the same figure, when possible. This is done for the CBF and pressures inside of each compartment, in order to better illustrate the differences between the simulation with mixed autoregulation and the previous simulations, in which the autoregulation has the same state in both hemispheres. Otherwise, in addition to the results, the difference of the simulated values of the mixed simulation with the corresponding values of the previous simulations are depicted for each hemisphere:

$$
\Delta_{l}^{k} X^{i}=\left.X^{i}\right|_{\text {case } k} ^{\text {mixed }}-\left.X^{i}\right|_{\text {case } l} ^{\text {identical }}
$$

whereas $i$ denotes the left or right hemisphere, and $k$ and $l$ are a placeholder for the number of the used case for the simulation. This is done in the following way: For the left hemisphere, the corresponding values of the simulation with disabled autoregulation in both hemispheres, are subtracted. The same is done in an analog way for the right hemisphere with enabled autoregulation. Due to its importance, this will also be done for the CBF. Here and in the following, identical in the context with autoregulation means, that for the autoregulation mechanism the same set of parameters is used. Therefore, when the autoregulation mechanism is identical, it is either en$\left(\alpha^{i}=1.4\right)$ or disabled $\left(\alpha^{i}=0\right)$.

It should be noted, that for the simulations, in which the autoregulation has the same state, the states in both hemispheres are identical. So in principal, it does not matter, which hemisphere is used. Also, for pressures and volume changes, only 7500 points in time with an offset of 3750 points in time are depicted, which still covers the whole ABP range. The reason for this is, that the splitting seen before in most of the graphs of the (e)sHOM model is not plotted. As the splitting distorts the information gained from the graph, it will not be depicted. This can be justified, as, on the one hand, the order of magnitude of the splitting can be determined by the previous simulations. On the other hand, no valuable information can be extracted from this splitting and can therefore be neglected.

\subsubsection{Case 5: no swelling, mixed autoregulation}

The CBF of each hemisphere for the case, in which there is no swelling, is depicted in figure 7.13, together with the CBF of the corresponding hemisphere of the simulations, in which the autoregulation has the same state in both sides of the brain. As for the left hemisphere, the results are compared with case 1, whereas for the right hemisphere, case 2 is used.

The difference between the CBF for mixed and identical autoregulation is relatively small for an ABP below $100 \mathrm{mmHg}$, which can directly be seen in figure 7.14. In the right hemisphere, the plateau, which stems from the 


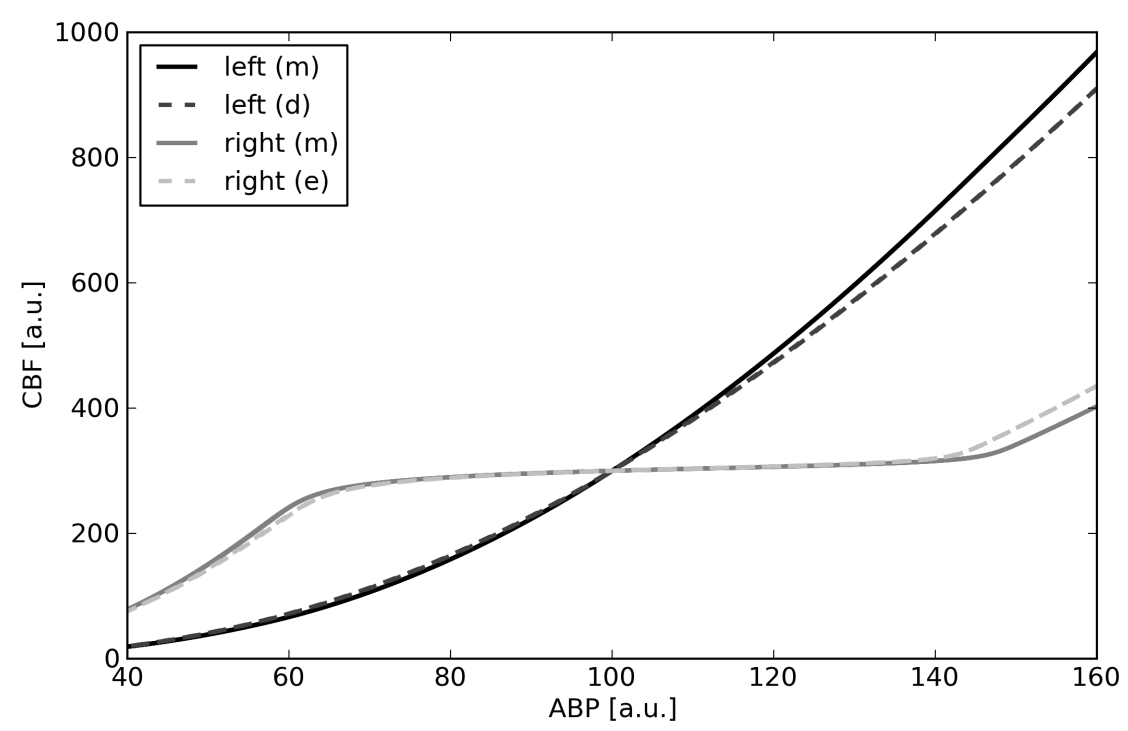

Figure 7.13: $\mathrm{CBF}$ against $\mathrm{ABP}$ with $V_{E}=0 \mathrm{ml}$; $(\mathrm{m})$ is used for mixed autoregulation, whereas for (d), autoregulation is disabled and for (e) enabled in both hemispheres (esHOM; case 5).

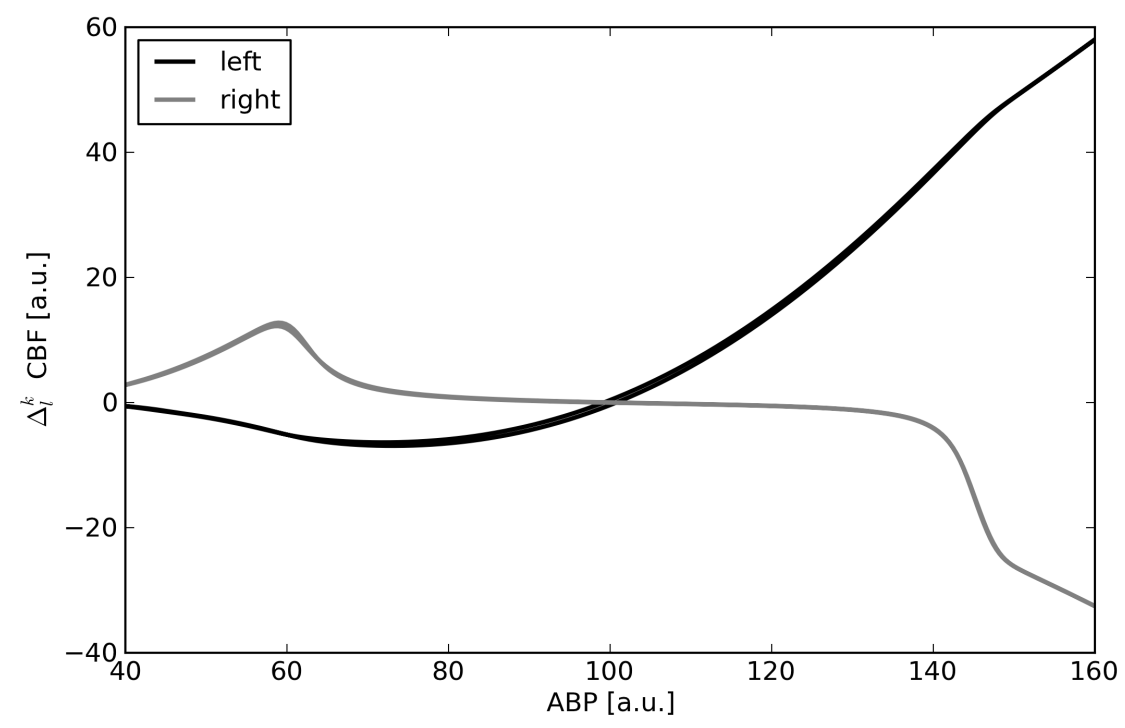

Figure 7.14: Difference of CBF between mixed and identical autoregulation against ABP for each hemisphere with $V_{E}=0 \mathrm{ml}$ (esHOM; case 5$)$. For $\Delta_{l}^{k}$, here, $k=5$, whereas $l=1$ (left) and $l=2$ (right). 
autoregulation, is slightly extended, but from there, the difference between both simulations decreases with decreasing ABP. As for the left hemisphere, the $\mathrm{CBF}$ decreases a little bit faster for decreasing ABP, until the difference reaches its minimum around an $\mathrm{ABP}$ of $70 \mathrm{mmHg}$, from where on the difference between the CBF in both simulations, decreases (see fig. 7.14).

For increasing ABP values above $100 \mathrm{mmHg}$, the difference between both simulations is more distinctive, although the graphs still have a similar slope. The CBF in the left hemisphere increases more rapidly, when compared to case 1 , in which the autoregulation was completely disabled. For the CBF in the right hemisphere, the autoregulation plateau is extended until an ABP of approx. $147 \mathrm{mmHg}$, as it was the case for decreasing ABP values below $100 \mathrm{mmHg}$. From there on, the increase in $\mathrm{CBF}$ in the right hemisphere is smaller for increasing ABP than it is for the other simulation (see fig. 7.14).

In addition, in figure 7.14 the new extremal points of the autoregulation mechanism at an ABP around 61 and $147 \mathrm{mmHg}$ are easy to identify. Outside of the autoregulation mechanism, the difference in CBF between both simulations has a quite linear course for the difference in the left hemisphere.

The reason for the changed CBF development in each hemisphere lies in the different volume development of the other hemisphere, when compared to the simulations with identical autoregulation on every side, and a different pressure development, as will be seen in the next sections.

\section{Pressure}

In figure 7.15 (left hemisphere) and 7.16 (right hemisphere) the pressures of the simulations with a mixed autoregulation $(\mathrm{m})$ and identical autoregulation (d) and (e) are depicted.

At first, the pressure development in the left hemisphere is discussed. Here, for ABP values below $100 \mathrm{mmHg}$, the pressure inside each compartment is slightly higher than in case 1 . The pressure difference of each compartment between both simulations reaches a maximum around an ABP of $64 \mathrm{mmHg}$. On the contrary, for ABP values above $100 \mathrm{mmHg}$ the pressure in each compartment is lower than before and drops in a first order approximation linearly, until it reaches the upper extremal autoregulation point at an $\mathrm{ABP}$ around $147 \mathrm{mmHg}$, from where on the slope of the difference in pressure is slightly reduced.

In the right hemisphere, the difference in pressure development is reverted. Here, for ABP values below $100 \mathrm{mmHg}$, the pressure inside each compartment is lower, when compared to the previous simulation (case 2) with identical autoregulation and is higher for $\mathrm{ABP}$ values above $100 \mathrm{mmHg}$. The correlation between the graphs of the pressures for the mixed and identical autoregulation is similar to the graphs, depicted in fig. 4.3, which shows the CBF for different $\alpha$. The effect of the hemisphere with disabled autoregulation on the other hemisphere, is in some way similar to a reduced $\alpha$ in 


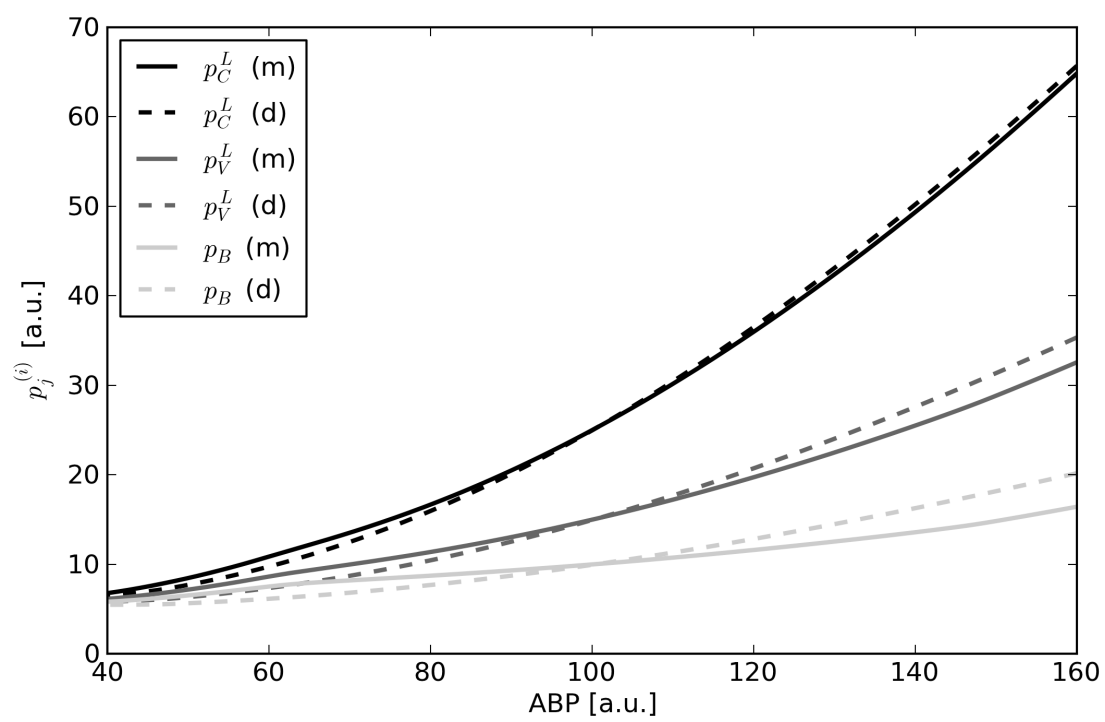

Figure 7.15: $p_{j}^{(i)}$ against ABP (left hemisphere) with $V_{E}=0 \mathrm{ml} ;(\mathrm{m})$ is used for mixed autoregulation and for (d), autoregulations is disabled in both hemispheres (esHOM; case 5).

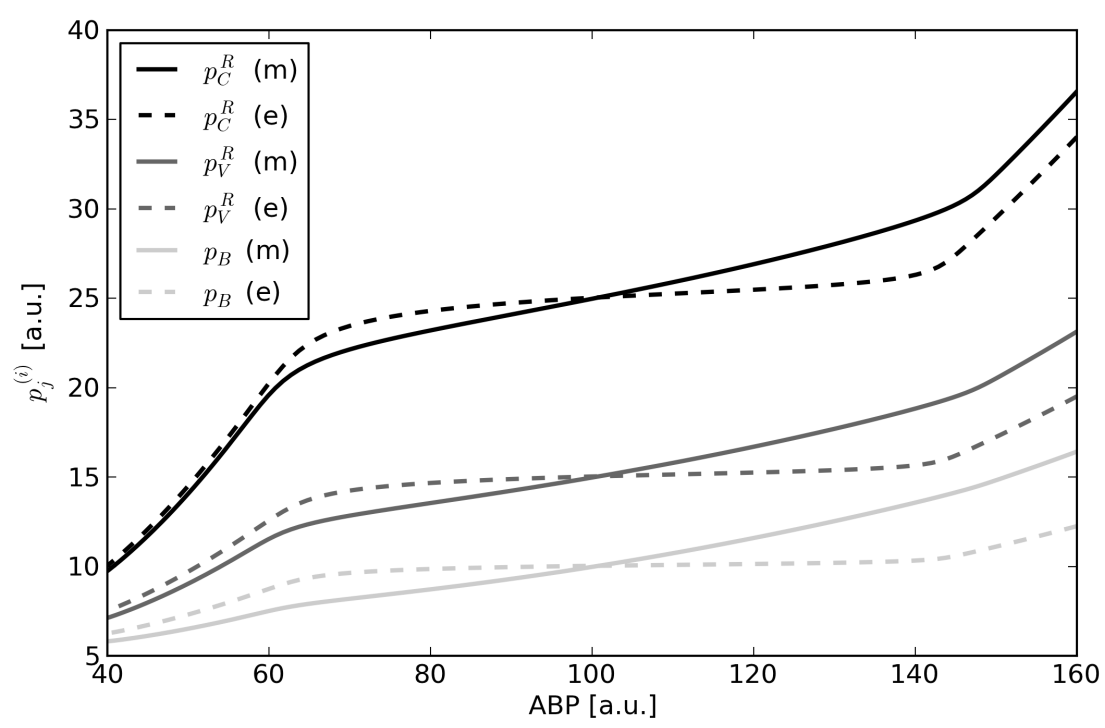

Figure 7.16: $p_{j}^{(i)}$ against $\mathrm{ABP}$ (right hemisphere) with $V_{E}=0 \mathrm{ml} ;(\mathrm{m})$ is used for mixed autoregulation and for (e), autoregulations is enabled in both hemispheres (esHOM; case 5). 
this hemisphere. The same holds the other way round, except that for the hemisphere with disabled autoregulation, $\alpha$ would be increased.

As before, the extremal autoregulation points at an ABP of around 61 and $147 \mathrm{mmHg}$ can be seen in fig. 7.16 and are also present in fig.7.15.

\section{Volume}

From the previous sections it can be deduced, that the difference in volume development should be relatively small, which will be seen in the following. At first, the volume development of the left hemisphere is discussed, which can be seen in figure 7.17, as its behavior is easier to explain. The difference in volume development between the simulations with mixed (case 5) and identical autoregulation (case 1) is depicted in 7.18.

For decreasing ABP values below $100 \mathrm{mmHg}$, all volumes decrease more rapidly in a nearly linear way, when compared to the corresponding simulation with identical autoregulation, until the lower autoregulation point is reached at an ABP at approx. $61 \mathrm{mmHg}$. The cause for the decreased volume development of all compartments in the left hemisphere lies in the active autoregulation in the right hemisphere. There, to maintain a steady $\mathrm{CBF}$, the arteries increase their volume with decreasing ABP. This results in a bigger volume for the sum of all blood vessels in the right hemisphere than in case 1. Therefore, the pressure inside the brain tissue is increased, when compared to case 1 , which in addition to the increased volume of the blood vessels in the right hemisphere is responsible for the decreased difference of volume in all compartments in the left hemisphere.

Below the lower extremal autoregulation point at an ABP of about $61 \mathrm{mmHg}$, the slope of the decrease changes its sign and the difference between both simulations is reduced with decreasing ABP. As the autoregulation has reached its limit, the volume of the blood vessels in the right hemisphere has no additional increase, due to the regulation mechanism. Therefore, the ICP decreases with decreasing ABP, as does the sum of the volumes of all blood vessels. With a decreasing ABP below the lower autoregulation point, the difference in volume of the blood vessels between both hemispheres is reduced and with it the difference in volume between all corresponding compartments between both hemispheres.

On the contrary, for increasing ABP values above $100 \mathrm{mmHg}$, all volumes increase more rapidly in a linear way, until the upper autoregulation point is reached. As before, responsible for this is the autoregulation in the right hemisphere in an analog way. But this time, the effect is reversed, as the diameter of the arteries is reduced, to maintain a stable $\mathrm{CBF}$.

Above the upper autoregulation point, the difference in volume for the veins and brain tissue is slightly increased, as is the volume difference of the arteries. This is due to the decreased sum of the volume of all blood vessels, when compared to the corresponding simulation with disabled autoregula- 


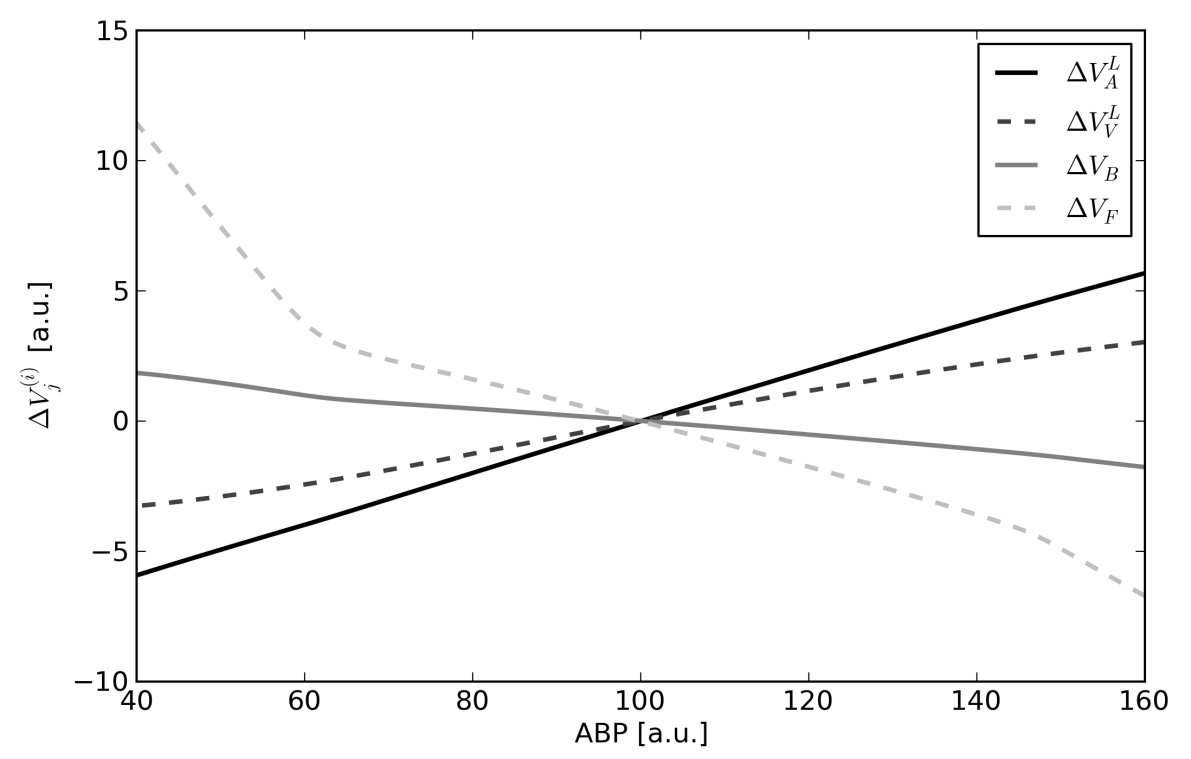

Figure 7.17: $\Delta V_{j}^{i}$ against $\mathrm{ABP}$ (left hemisphere) with $V_{E}=0 \mathrm{ml}$ (esHOM; case 5$)$.

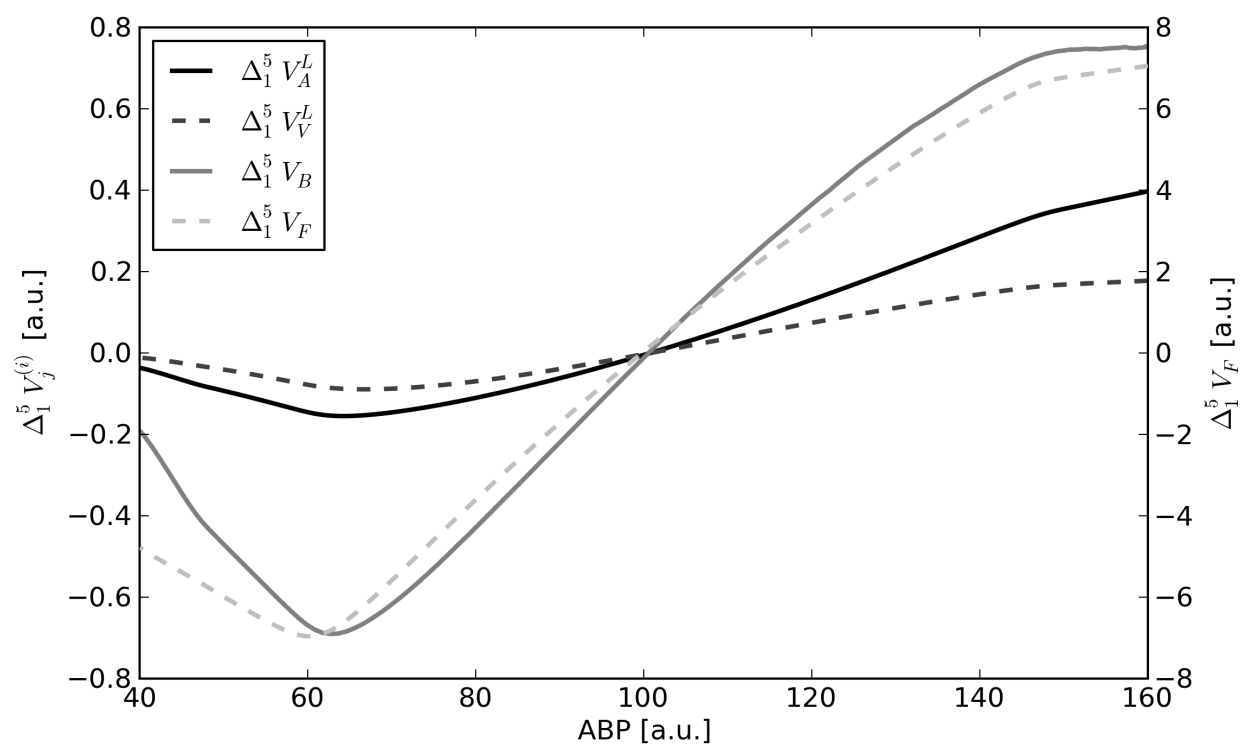

Figure 7.18: ABP against $\Delta_{1}^{5} V_{j}^{i}$ (left hemisphere) with $V_{E}=0 \mathrm{ml}$ (esHOM; case 5$). \Delta_{1}^{5} V_{F}$ is plotted on a separate $y$-axis (right). 
tion in both hemispheres (case 1). Therefore, the ICP is smaller, due to a reduced compression of the brain tissue, and therefore results in an increase in volume of the blood vessels in the left hemisphere.

The difference of the volume development of the CSF fluid is shown for completeness. But an interpretation of its development is omitted, as it depends on the volume development of both hemispheres. An interpretation in this context with only one hemisphere can be misleading, as for its development more factors than just the difference between the previous simulations have to be taken into account. The only interesting aspect is, that the volume changes of all other compartments can be compensated by the CSF compartment, as it is never fully depleted.

As for the volume developments in the right hemisphere, the graphs have a more complicated course and are shown in fig. 7.19, whereas the differences in volume development between case 5 and 2 are depicted in fig. 7.20. The graph depicting the volume difference for the brain tissue has more or less the same course as in the left hemisphere, but with different sign. The same holds for the difference of CSF.

Inside the autoregulation range, the volume of the veins is practically identical to the corresponding simulation, done in case 2. The reason for this is, that the pressure difference between veins and brain tissue stays the same (compare fig. 7.16). At the lower limit of the autoregulation, the volume difference increases and reaches a maximum around an ABP value of $60 \mathrm{mmHg}$, from where on the difference decreases with decreasing ABP (see fig. 7.20). This is due to the faster decrease of the ICP in case 5 than in case 2 . In addition, the ICP decreases faster than the pressure inside the veins, as the volume of all blood vessels in both hemispheres decreases with decreasing ABP.

Around the upper autoregulation point, the previously described process happens in an analog way, but here, the increase of the volume of the veins is reduced, contrary to the former simulation done in case 2 . This is due to the higher ICP in the right hemisphere, when compared to the corresponding simulation with identical autoregulation (compare section 7.2.2).

The change in the volume development of the arteries is the most complex one. As the pressure inside the capillaries in the right hemisphere is smaller than in case 2 of the previous simulation for decreasing ABP below $100 \mathrm{mmHg}$, the compliance of the arteries must be altered less, due to the already increased difference $p_{A}-p_{C}$. Therefore, the necessary dilation of the arteries is smaller than in case 2 . This is also the reason, why the autoregulation plateau can be elongated, as the full dilation is reached at a lower ABP value. After passing the lower autoregulation point of case 2, the dilations continues, until the new lower autoregulation point is reached at an ABP around $61 \mathrm{mmHg}$. From there onwards, due to a in general decreased ICP in the right hemisphere and with it a reduced pressure difference between $\mathrm{ABP}$ and ICP, the volume of the arteries remains bigger, when compared to 


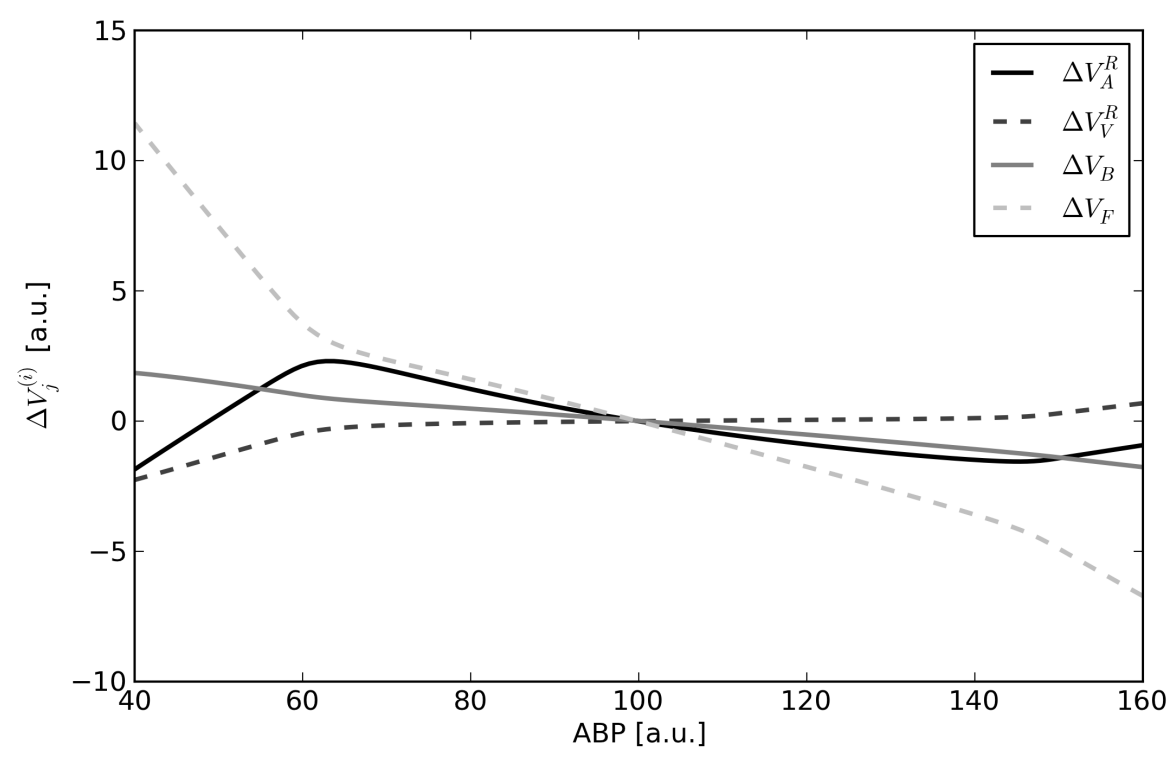

Figure 7.19: $\Delta V_{j}^{i}$ against $\mathrm{ABP}$ (right hemisphere) with $V_{E}=0 \mathrm{ml}$ (esHOM; case 5).

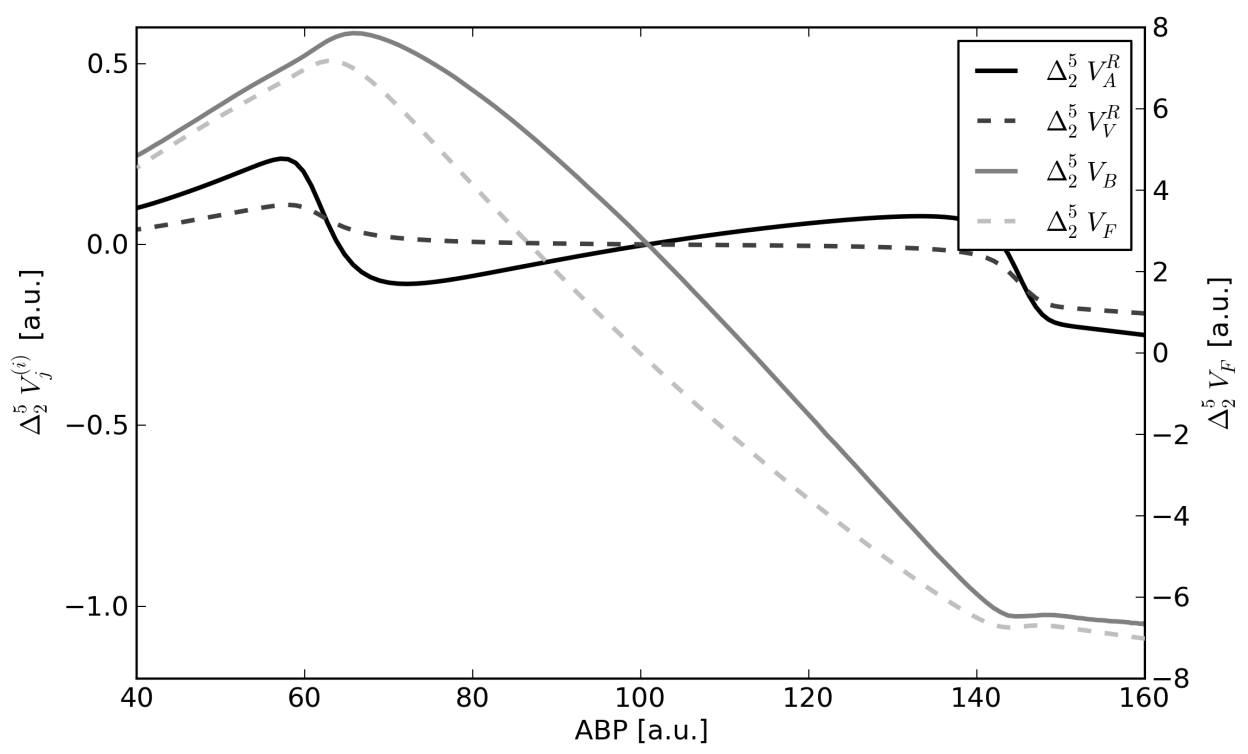

Figure 7.20: $\Delta_{2}^{5} V_{j}^{i}$ against ABP (right hemisphere) with $V_{E}=0 \mathrm{ml}$ (esHOM; case 5). $\Delta_{2}^{5} V_{F}$ is plotted on a separate $y$-axis (right). 
the previous simulations in case 2 .

The process happens in an analog way for an increasing ABP above $100 \mathrm{mmHg}$ inside the autoregulation range, but with different sign. For increasing $\mathrm{ABP}$ values above the upper autoregulation point, the volume of the arteries decreases less than in case 2. The compliance $C_{A B}^{R}$ has to be altered less, due to the increased ICP. The reason for the increase of the ICP, when compared to case 2 , is the increased total volume of all blood vessels.

\subsubsection{Case 6: swelling, mixed autoregulation}

From the previous simulations it can be assumed, that a swelling should have a much bigger effect on the coupling between both hemispheres, than the one, which has been seen in section 7.4.1.

The altered CBFs in each hemisphere in addition to the corresponding CBFs of the simulations with identical autoregulation can be seen in figure 7.21. The difference between the simulations with mixed and identical autoregulation can be seen in figure 7.22. The left hemisphere is compared to case 3 , whereas for the right hemisphere, case 4 is used.

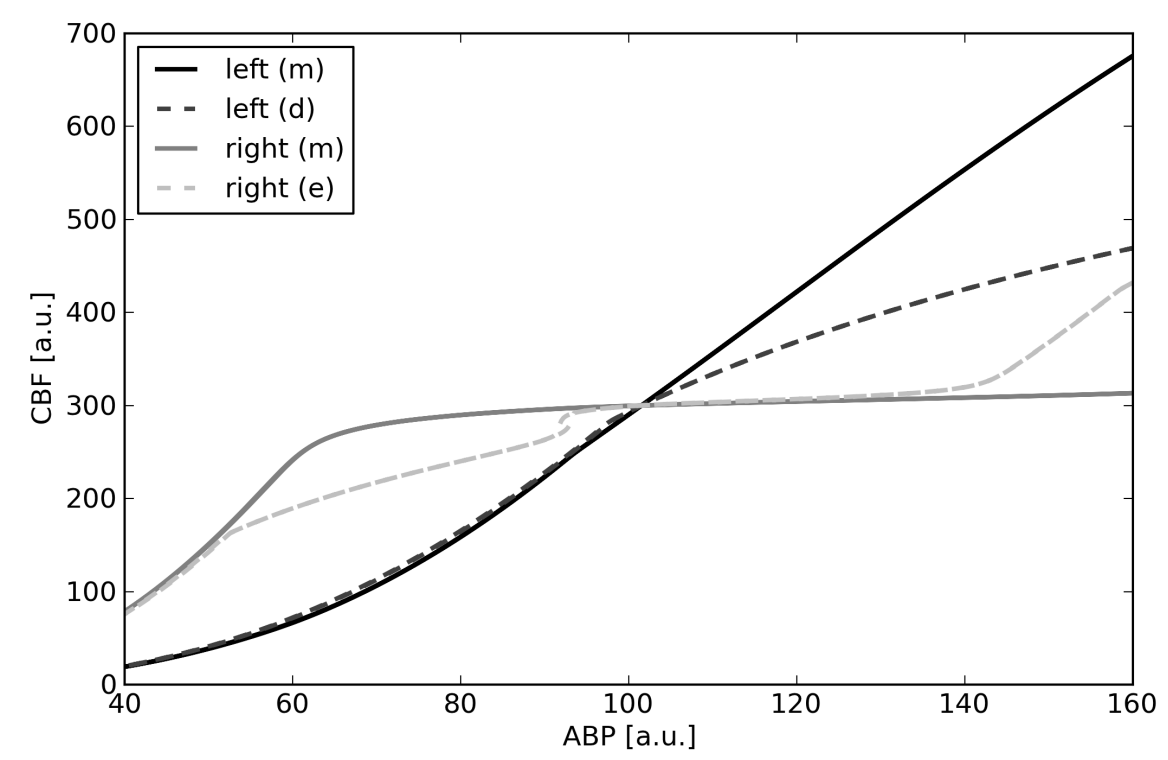

Figure 7.21: CBF against ABP with $V_{E}=30 \mathrm{ml} ;(\mathrm{m})$ is used for mixed autoregulation, whereas for (d), autoregulation is disabled and for (e) enabled in both hemispheres (esHOM; case 6).

First, the effect of the swelling on the coupling between both hemispheres 


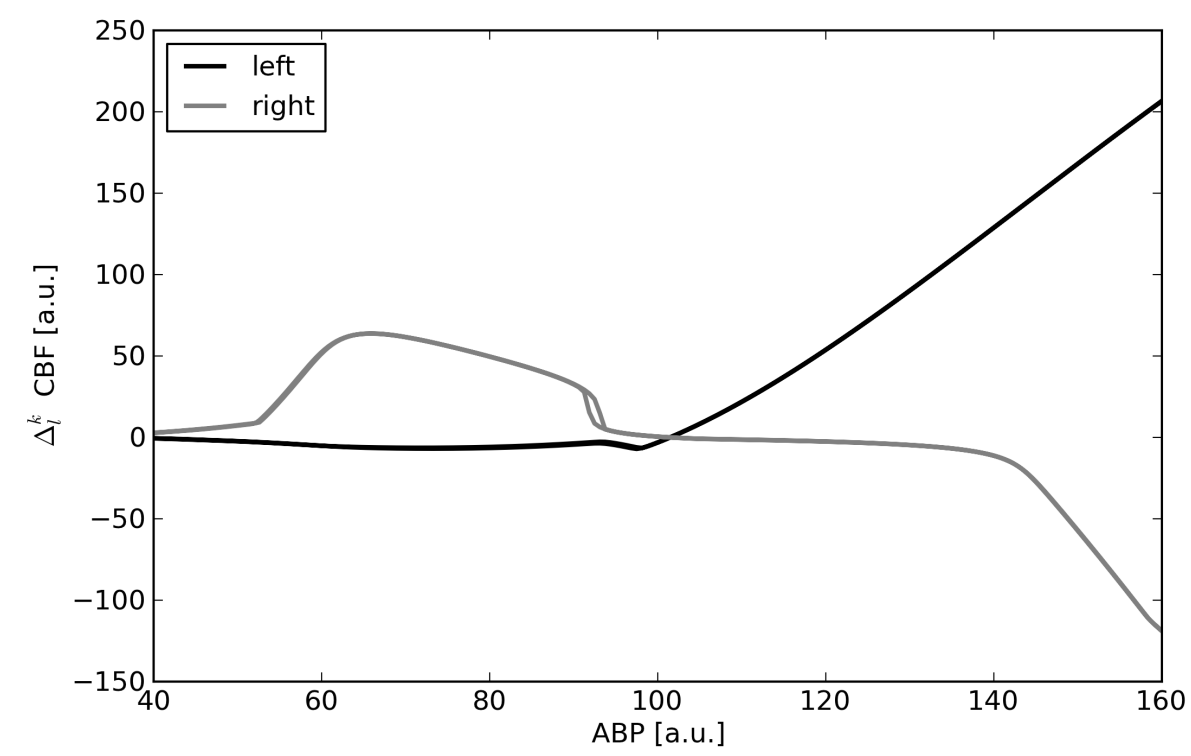

Figure 7.22: Difference of CBF between mixed and identical autoregulation against $\mathrm{ABP}$ for each hemisphere with $V_{E}=30 \mathrm{ml}$ (esHOM; case 6). For $\Delta_{l}^{k}$, here, $k=6$, whereas $l=3$ (left) and $l=4$ (right).

is examined for the left hemisphere, in which the autoregulation is disabled. As for ABP values below $100 \mathrm{mmHg}$, both curves have a similar run. For higher ABP values the graphs between both simulations differ. Here, the increase of the $\mathrm{CBF}$ in the left hemisphere is much higher and is more comparable to the simulations done with the bHOM model.

A similar result can be seen for the $\mathrm{CBF}$ in the right hemisphere. Here, when compared with the corresponding simulation (case 4), the drop in $\mathrm{CBF}$ vanishes for an ABP below $100 \mathrm{mmHg}$. For higher ABP values, the upper part of the autoregulation plateau is extended over the whole upper $\mathrm{ABP}$ range. Because of its shape, the whole course of the $\mathrm{CBF}$ in the right hemisphere is more similar to the $\mathrm{CBF}$ of the bHOM model than to the sHOM model.

\section{Pressure}

The pressure curves for mixed and identical autoregulation are shown in figure 7.23 (left hemisphere) and 7.24 (right hemisphere).

For ABP values below $100 \mathrm{mmHg}$, there are only small deviations between both simulations for the left hemispheres, similar to the changes seen in case 5. For increasing $\mathrm{ABP}$ values above $100 \mathrm{mmHg}$, the pressures in- 


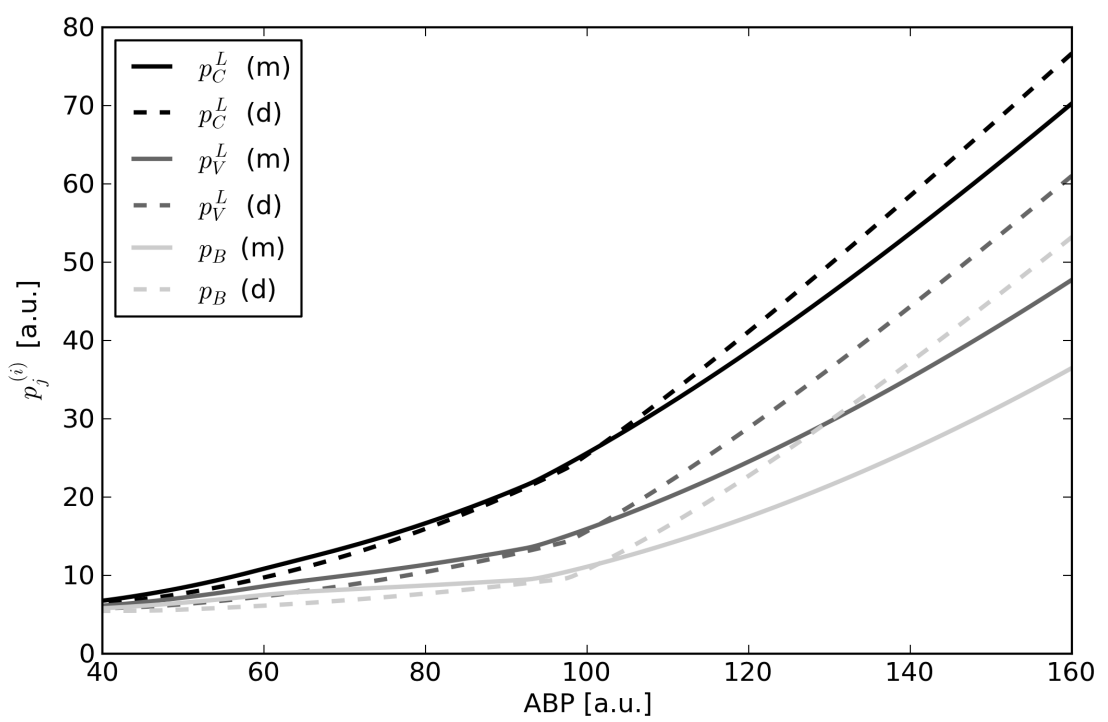

Figure 7.23: $p_{j}^{(i)}$ against ABP (left hemisphere) with $V_{E}=30 \mathrm{ml} ;(\mathrm{m})$ is used for mixed autoregulation and for (d), autoregulations is disabled in both hemispheres (esHOM; case 6).

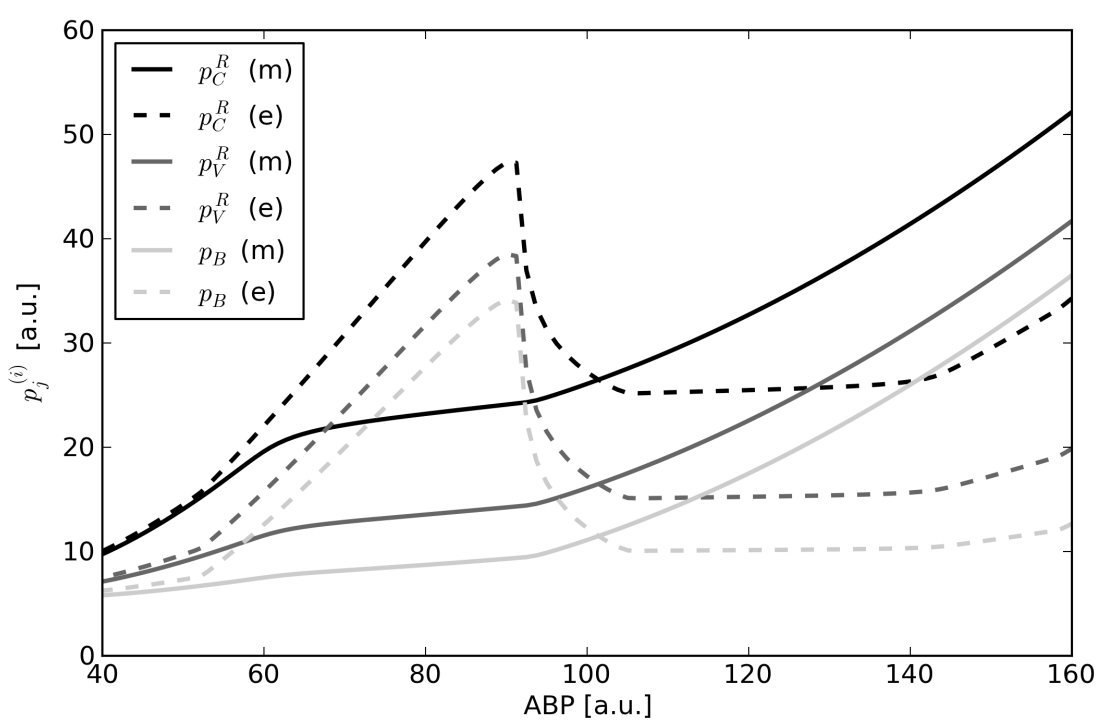

Figure 7.24: $p_{j}^{(i)}$ against ABP (right hemisphere) with $V_{E}=30 \mathrm{ml} ;(\mathrm{m})$ is used for mixed autoregulation and for (e), autoregulations is enabled in both hemispheres (esHOM; case 6). 
crease slower for the mixed autoregulation simulation, than they increase in the previous simulations (case 3 ).

As for the right hemisphere, here, the big increase in pressure for all compartments for the simulation, seen in case 4, vanishes. For ABP values above $105 \mathrm{mmHg}$, the pressures in the mixed autoregulation simulation are at first slightly higher (around $4 \mathrm{mmHg}$ ) and have an additional linear increase onwards.

\section{Volume}

The volume development in each hemisphere is much more complicated, than in the previous case, in which there was no swelling. The differences in volume development between the mixed and identical autoregulation simulations are depicted in figure 7.25 (right hemisphere) and 7.27 (left hemisphere). Additionally, the differences in volume development between both simulations are depicted in 7.26 (right hemisphere) and 7.28 (left hemisphere).

From figure 7.25 it can be deduced, that the CSF compartment is completely depleted for ABP values above $95 \mathrm{mmHg}$, similar to case 3 .

For the case, that there is still CSF left to absorb, this yields the following behavior: In the right hemisphere, the development of all compartments is similar to case 2. This is not what is depicted in figure 7.26, as here, the results are compared to case 4 . In case 4 , for ABP values between 55 and $105 \mathrm{mmHg}$, the CSF compartment was depleted. Both, the differences in CSF production and autoregulation, can be seen quite clearly in the graphs. But as long as there is CSF to absorb (40 to $55 \mathrm{mmHg} \mathrm{ABP}$ ), the simulations in case 4 and here have similar results. In the left hemisphere, the development of all compartments is similar to case 5, except for the CSF compartment, due to the different volume development in the right hemisphere.

As already discussed in section 7.3.2, due to the depletion in CSF for ABP values below $95 \mathrm{mmHg}$, in case 4, the autoregulation of the arteries reaches its lower extremal autoregulation point at an $\mathrm{ABP}$ value of around $95 \mathrm{mmHg}$. This can be seen in figure 7.26 , where the difference in volume development of the arteries drops in the right hemisphere. Here, the lower autoregulation point is reached at around an $\mathrm{ABP}$ of $60 \mathrm{mmHg}$, which results in an increase of the volume of the arteries (compare fig. 7.25), until the lower extremal autoregulation point is reached. The difference in autoregulation is also reflected in the development of the veins. Due to the smaller total volume of all blood vessels in both hemispheres, the brain tissue is less compressed, which can also be seen in figure 7.26.

As for the difference in volume development for an ABP above $97 \mathrm{mmHg}$, first, the right hemisphere is discussed. For ABP values between 97 to $105 \mathrm{mmHg}$, the CSF compartment is depleted in both simulations. The 


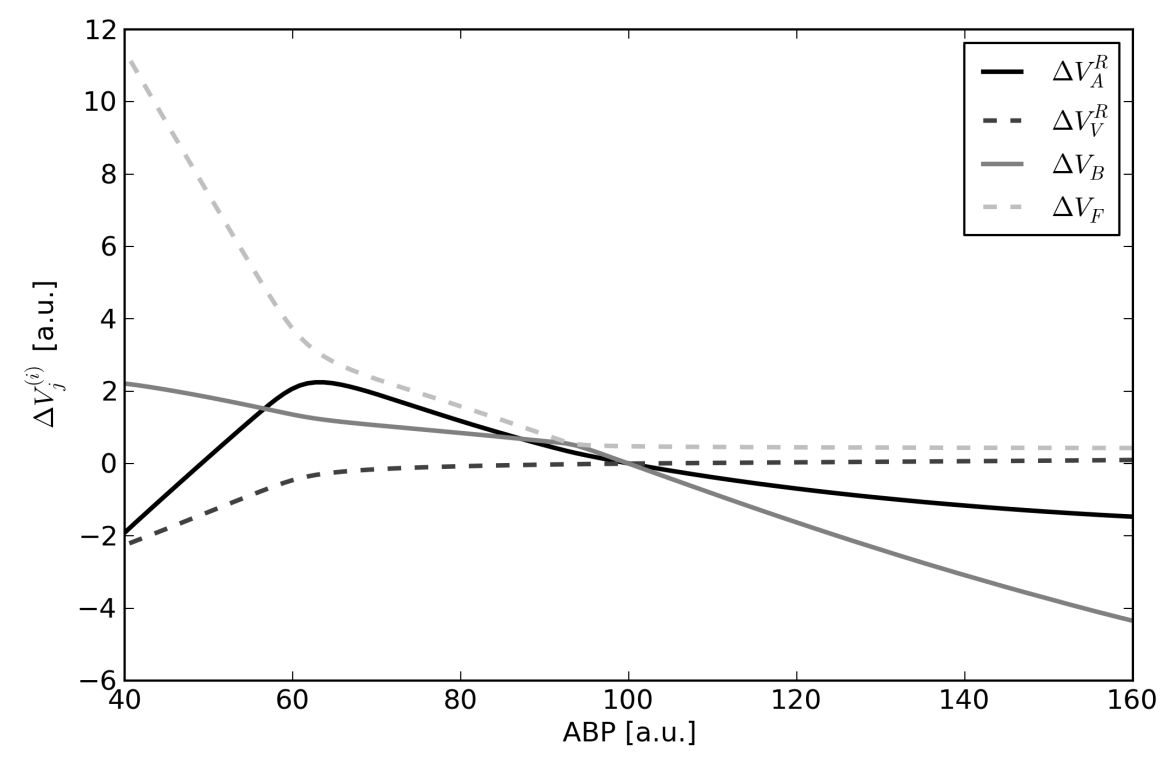

Figure 7.25: $\Delta V_{j}^{i}$ against $\mathrm{ABP}$ (right hemisphere) with $V_{E}=30 \mathrm{ml}$ (esHOM; case 6$)$.

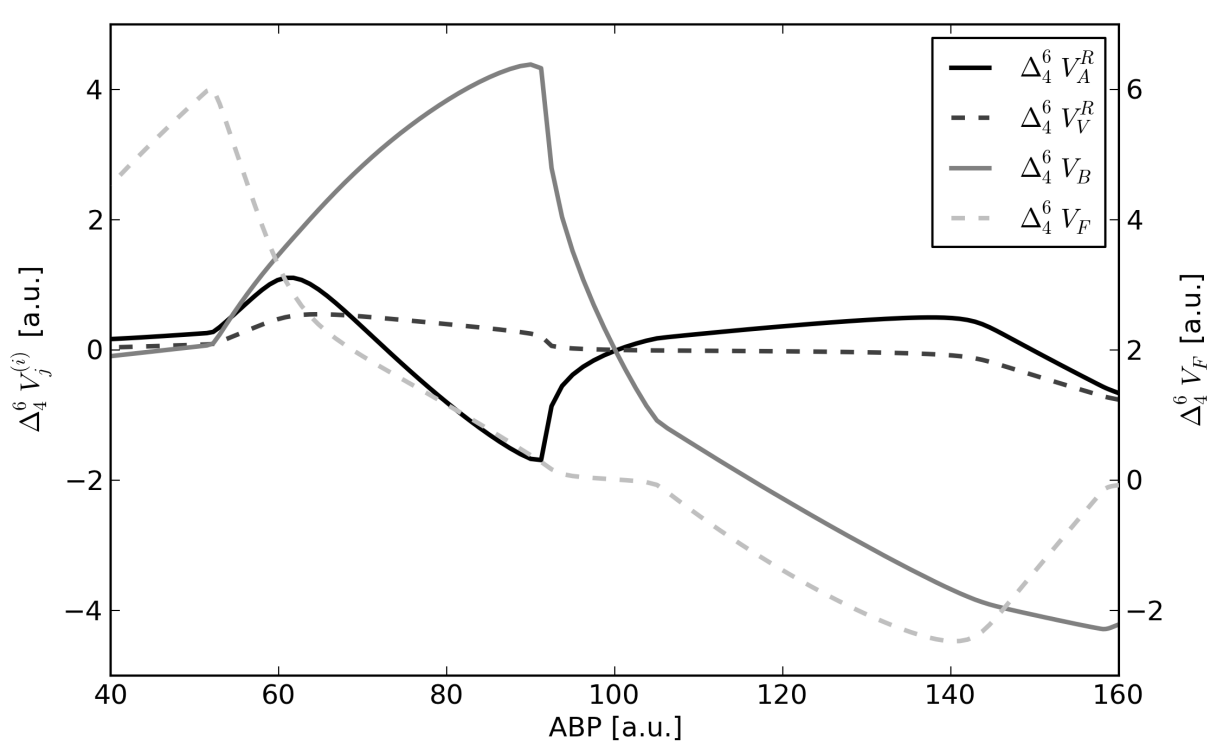

Figure 7.26: $\Delta_{4}^{6} V_{j}^{i}$ against ABP (right hemisphere) with $V_{E}=30 \mathrm{ml}$ (esHOM; case 6 ). $\Delta_{4}^{6} V_{F}$ is plotted on a separate $y$-axis (right). 


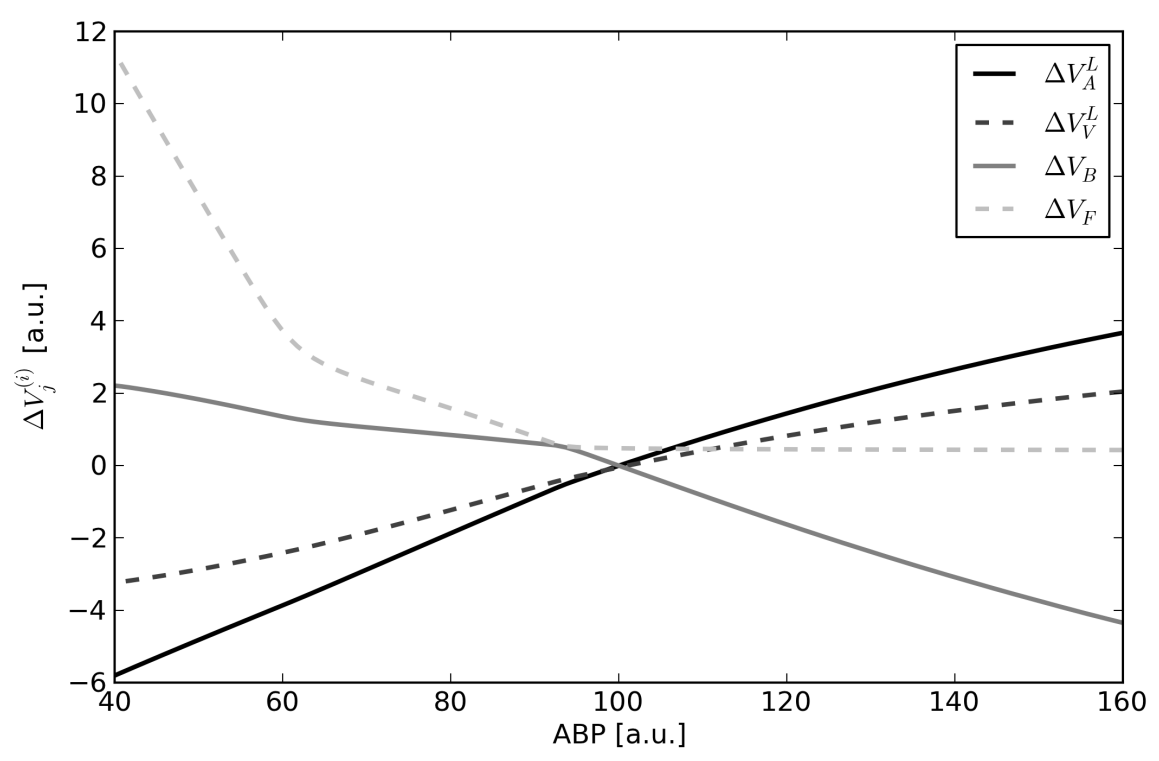

Figure 7.27: $\Delta V_{j}^{i}$ against ABP (left hemisphere) with $V_{E}=30 \mathrm{ml}$ (esHOM; case 6$)$.

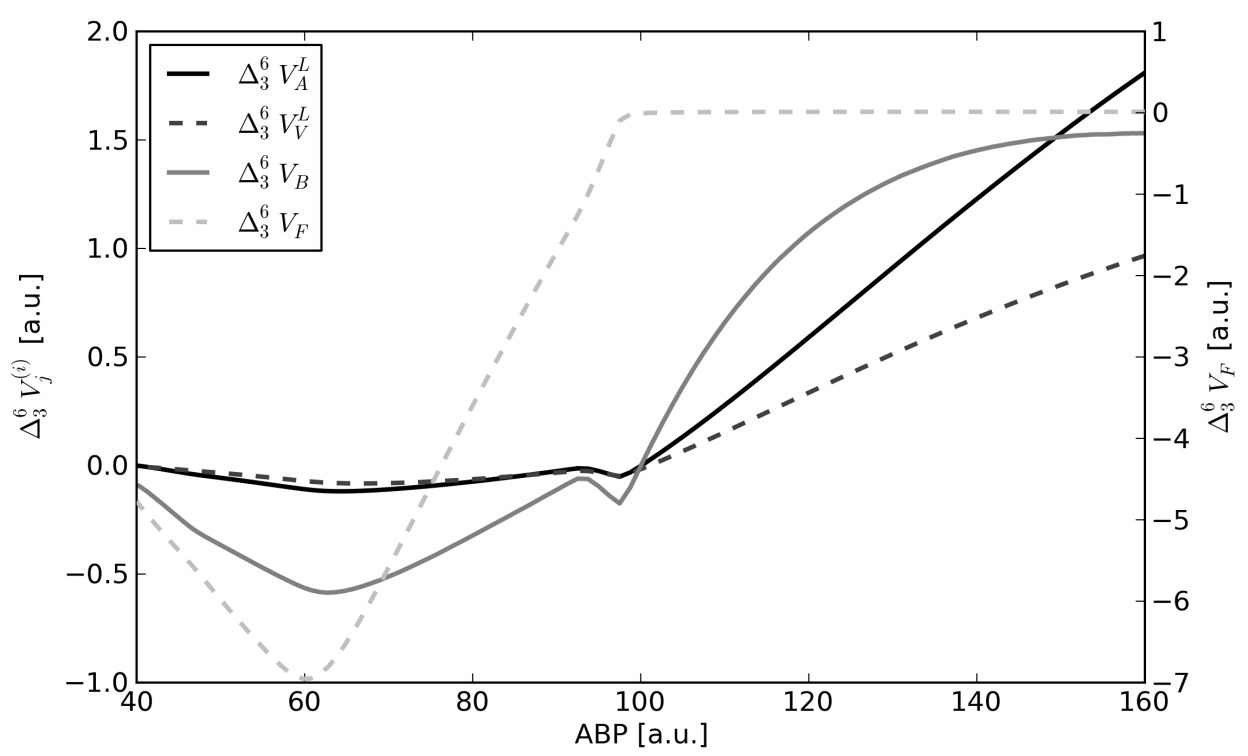

Figure 7.28: $\Delta_{3}^{6} V_{j}^{i}$ against ABP (left hemisphere) with $V_{E}=30 \mathrm{ml}$ (esHOM; case 6$). \Delta_{3}^{6} V_{F}$ is plotted on a separate $y$-axis (right). 
differences, seen inside this segment in fig. 7.26, stem from the different total volume of the blood vessels in the left hemisphere. Here, the total volume of the blood vessels below an ABP of $100 \mathrm{mmHg}$ is smaller than in case 4 and bigger for an ABP above $100 \mathrm{mmHg}$. This can be seen in the corresponding graphs, depicting the volume change of the arteries and brain tissue. As for the veins and CSF compartment, both graphs have constant values inside this range. The reason for this behavior of the CSF compartment is clear, because in both scenarios, it is depleted. As for the veins, because of the same development of the pressure inside the veins and brain tissue, the difference between both is constant, which results in the constant volume difference.

For ABP values between an ABP of around 105 to $140 \mathrm{mmHg}$, there is no difference between the volume development of the veins, because of the same pressure difference between veins and brain tissue in both simulations. The volume of the arteries in the right hemisphere is slightly increased. The reason for this are the increased pressures inside each compartment in the right hemisphere, due to the depletion of the CSF compartment. Therefore, the adjustment of the compliance of the arteries, due to the autoregulation mechanism, is smaller (compare previous section). As there is no CSF left to absorb, the brain tissue gets more compressed with increasing volume of the blood vessels.

Above an ABP of $140 \mathrm{mmHg}$, contrary to the corresponding simulation (case 4), the upper extremal autoregulation point is still not reached, as the necessary adjustment to $C_{A B}^{R}$ is smaller. Therefore, the difference in volume of the arteries and veins drops, as the total volume of the blood vessels in the corresponding simulation (case 4) raises in the right hemisphere, but here, the corresponding total volume decreases. The difference in volume of the brain tissue is still smaller, which is because of the still depleted CSF compartment and a bigger total volume of all blood vessels as in case 4 .

In the left hemisphere, for ABP values above $100 \mathrm{mmHg}$, the difference in volume development stems from the depleted CSF compartment and autoregulation in the right hemisphere.

As the upper extremal autoregulation lies outside of the maximal ABP value, the volume of the arteries in the right hemisphere decreases in a more or less linear way with increasing ABP, in order to keep a constant CBF. As the total volume of the blood vessels in the right hemisphere decreases with increasing $\mathrm{ABP}$, the volume of the veins and arteries in the left hemisphere show a nearly linear but smaller increase, too. Although the CSF compartment is depleted, due the aforementioned decrease in volume of the blood vessel system in the right hemisphere, the ICP inside the left hemisphere is smaller than in case 3 . Therefore, the difference in volume of the brain tissue increases for the left hemisphere. With increasing ABP, the increase for the brain tissue flattens and becomes nearly constant for a maximal ABP.

For ABP values between 93 and $100 \mathrm{mmHg}$, the drop in volume difference 
for all compartments in the left hemisphere reaches its minimum at the start of the depletion of the CSF compartment, which is around an ABP of $97 \mathrm{mmHg}$. Responsible for the drop is the increased ICP, as the positive volume change of all blood vessels, due to the autoregulation, cannot be compensated by the depleted CSF compartment. With decreasing ABP below $97 \mathrm{mmHg}$, the differences decrease and converge to the development, seen in the previous simulations.

\subsection{Summary}

In this chapter, the effect of splitting the model into two hemispheres was examined and compared to the sHOM model. As for the simulations with and without enabled autoregulation, but without a swelling, there are no significant differences in the CBF of the whole brain. Due to the adjustments, resistances and compliances of divided compartments differ only by a factor, given in section 4.4.1.

The main difference between both models lies in the different volume development of the compartments. The compliance of the arteries has been adjusted in such a way, that the $\mathrm{CBF}$ in the models is as similar to each other as possible. The outcome of this adjustment is, that the volume change of each artery compartment in the esHOM model is smaller than in the sHOM model. But the absolute volume change of the sum of both artery compartments is bigger. As for the veins, due to the same used compliance and similar pressures as in the sHOM model, the volume change of each compartment is practically identical to the development of the venous compartment of the sHOM model. But therefore, for the whole venous compartment in the esHOM model the volume change is twice as big. This is due to the design of the esHOM model, for which the intention was, to alter as much as necessary, in order to separate the brain model into two hemisphere, but leave the rest as is. The important aspect of the first two simulations is, that the blood vessel system can, depending on the pressures inside each compartment, use much more space than in the sHOM model.

As for case 3 and 4 , in which a swelling was simulated, the CBF between sHOM and esHOM model is similar as long as there is CSF left to absorb. Inside the segment, in which the CSF compartment is depleted, both models differ a lot, mainly due to the different volume development of the blood vessels. In case 3 , for $\mathrm{ABP}$ values above approx. $95 \mathrm{mmHg}$, the $\mathrm{CBF}$ increases slower in the esHOM model than in the sHOM model. As the blood vessels occupy more space than in the sHOM model, the pressure inside the capillaries, veins and brain tissue increases. This ultimately results in the decreased $\mathrm{CBF}$, when compared to the CBF of the sHOM model.

In principal, the same holds for case 4 , but there, the situation is slightly more complicated, due to the autoregulation mechanism. There, the effect, 
seen in the CBF development of the sHOM model, is enhanced, as the arteries try to compensate the drop in CBF. Therefore, they have to increase their volume, in order to reduce their resistance. But as this increase can no longer be compensated by the depleted CSF compartment, it results in an increase of ICP and all other pressures, which hampers the CBF. This is the cause for the premature reaching of the maximal dilation of the arteries. From there on, the volume of the blood vessels decreases with decreasing $\mathrm{ABP}$, which reduces the compression of the brain tissue. But all pressures are still higher, when compared to the sHOM model. At an ABP of approx. $50 \mathrm{mmHg}$, the situation normalizes and both models behave comparably again.

Case 5 and 6 were only done for the esHOM model, as there are no corresponding scenarios for the bHOM and sHOM models. Here, the simulations were done with a disabled autoregulation in one hemisphere and enabled autoregulation in the other. This was done without (case 5) and with (case 6 ) a simulated swelling. The reason for this simulation was, to see, how both hemispheres are coupled with each other, whereas the coupling is induced by the brain tissue.

As for case 5, the coupling was minimal. There are small differences in the development of the CBF. For the hemisphere with disabled autoregulation, the $\mathrm{CBF}$ was slightly increased below an $\mathrm{ABP}$ of $100 \mathrm{mmHg}$, and slightly decreased above, when compared to case 1 . As for the hemisphere with enabled autoregulation, the CBF plateau, induced by the autoregulation mechanism, was elongated. In a first order approximation, the effect of the coupling may be described by an adjustment of $\alpha$ in each hemisphere towards $\alpha$ in the other hemisphere.

In the last case, the coupling was examined for the scenario, in which a swelling was simulated. Here, the CSF compartment was depleted for an ABP higher than $95 \mathrm{mmHg}$. Contrary to case 5 , in this scenario the effect of the coupling is much higher. Due to the enabled autoregulation in one hemisphere, the total volume of the blood vessels is reduced for an ABP above $95 \mathrm{mmHg}$, which results in a decreased ICP in the hemisphere with disabled autoregulation, compared to case 3 . This is the reason, why the $\mathrm{CBF}$ in this hemisphere is increased. For the hemisphere with enabled autoregulation, the CBF can be stabilized longer, as the ICP is increased, when compared to case 4 . Due to the increased ICP, the compliance of the arteries is already reduced and with it the volume of the arteries, without active regulation. Therefore, the necessary regulation of the autoregulation is smaller, which means, that the point of maximal constriction is reached for higher ABP values. 


\section{Chapter 8}

\section{Evaluation of the models}

In the previous chapters, the haemodynamical processes of the human brain were simulated with different models and examined in detail. The models allow to get a better understanding, how the underlying mechanisms, as the autoregulation and cerebrospinal fluid (CSF) production, influence the cerebral blood flow (CBF) of the brain.

Unfortunately, the CBF itself is a value, which is hard to measure. Measurements, which are done on patients with a severe head injury, include among others the intracranial pressure (ICP) and partial oxygen pressure in tissue $\left(\mathrm{TiPO}_{2}\right)$. But the last observable can be simulated, using the simulated $\mathrm{CBF}$ and the model for calculating the oxygen transport, introduced in the second part of chapter 3 .

Therefore, in this chapter, the oxygen transport into the tissue is calculated for the different simulations, done so far. In addition, the simulated ICP will also be depicted, as both observables represents parameters, that are measured during neuromonitoring. As for this part, the basic Haemodynamical and Oxygen transport Model (bHOM) is omitted in this discussion, due to the reasons given in the previous chapters.

As all different types of models are able to reproduce known behavior of haemodynamical processes, in the last section all models are used to interpret a time segment of real measured data from patients with a severe head injury. The physiological background of the correlation between the shown measured values, namely the arterial blood pressure (ABP), ICP and $\mathrm{TiPO}_{2}$, is still quite ambiguous. In the measured data the partial oxygen pressure in tissue is anti-correlated to the mean arterial blood pressure and intracranial pressure, which are both correlated. But this kind of behavior can be seen in around $66 \%$ of the patient specific data sets (compare [5]) and can therefore be assumed as relevant scenario for this type of patients.

It should be mentioned, that measured data represents a local measurement, whereas the simulations can generate only results for the whole brain and each hemisphere, respectively. For example, local variations of a global 
state could be generated, induced by variation of the local autoregulation inside a smaller section of the human brain, due to a different local consumption of oxygen. Only, if there are no local phenomena or ones with small effects, the simulations will be able to reproduce measured behavior.

\section{1 sHOM model}

As before, at first, the graphs for the ICP and partial oxygen pressure in tissue are discussed for the sHOM model, whereas the results for the esHOM model will be discussed in section 8.2.

\subsubsection{Case 1: no swelling, no autoregulation}

In figure 8.1, the simulated partial oxygen pressure in tissue $\left(\mathrm{TiPO}_{2}\right)$ as well as the brain pressure (ICP) are plotted against the arterial blood pressure $(\mathrm{ABP})$.

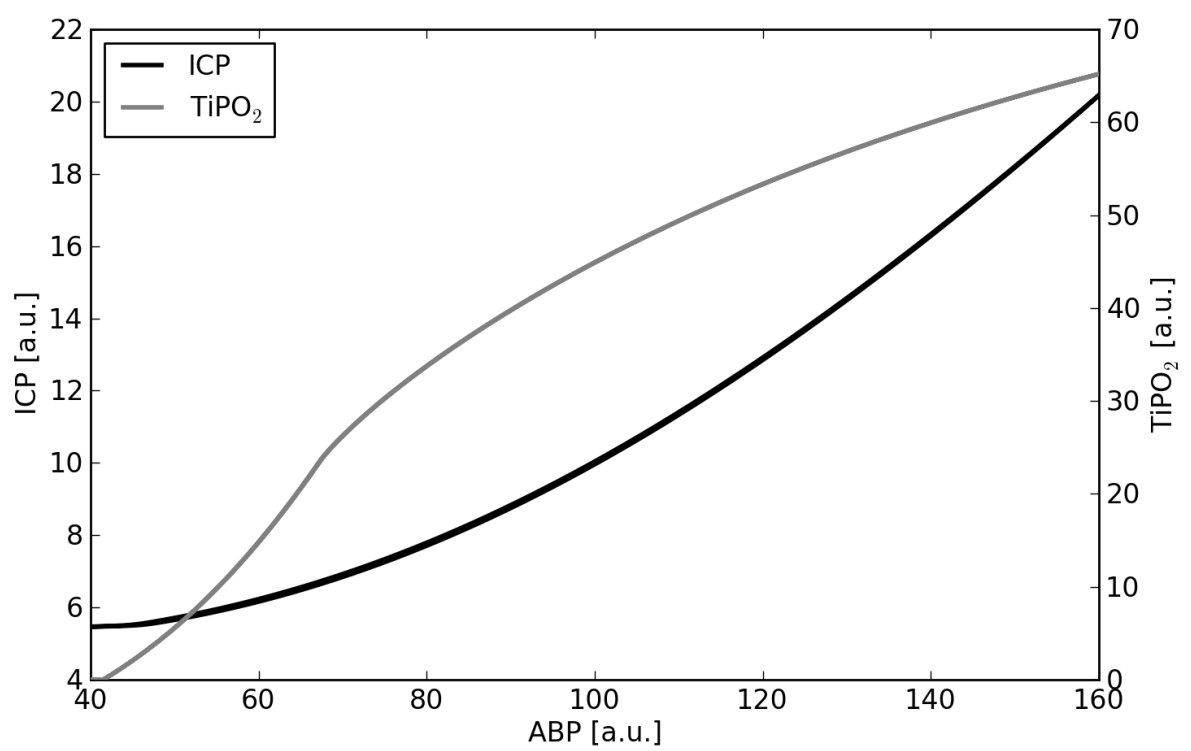

Figure 8.1: $\mathrm{TiPO}_{2}$ and ICP against ABP $\alpha=0.0$ and $V_{E}=0 \mathrm{ml}(\mathrm{sHOM}$; case 1$)$.

When compared directly with the corresponding CBF (seen in fig. 6.1), the run of the $\mathrm{TiPO}_{2}$ and $\mathrm{CBF}$ graph differ. Here, both the run of the CBF and the ICP have a similar slope, with respect to the curvature, but the $\mathrm{TiPO}_{2}$ looks quite different. 
First, for ABP values below $42 \mathrm{mmHg}$, the partial oxygen pressure equals zero, which means, that all oxygen is consumed that is transported into the tissue. From there on, the graph can be separated into two parts. Between an $\mathrm{ABP}$ of 42 and $68 \mathrm{mmHg}$, there is an rapid increase in $\mathrm{TiPO}_{2}$ with a similar slope as the increase of CBF or ICP. But above an ABP of $68 \mathrm{mmHg}$, the convex increase changes into a concave increase: with a linear increase in $\mathrm{ABP}$ the increase in $\mathrm{TiPO}_{2}$ decreases.

From equation 3.36 it is clear, that the diffusion into the tissue depends on the partial oxygen pressure in blood $\left(\mathrm{pbO}_{2}\right)$. As can be seen from equation $3.28, \mathrm{pbO}_{2}$ depends on the velocity of blood $v$, which is directly proportional to the $\mathrm{CBF}$.

As the CBF increases inside the whole ABP range, so does the $\mathrm{TiPO}_{2}$, with the aforementioned exception below $42 \mathrm{mmHg}$. Responsible for the concave development of the $\mathrm{TiPO}_{2}$ is the buffer like property of the saturated haemoglobin. As long as there is more oxygen in the system than the amount, which is consumed, the increase in $\mathrm{pbO}_{2}$ will be small with increasing CBF.

This behavior will change, when the oxygen buffers start to become depleted. From the graphs it can be seen, that this starts to happen in the simulations around an ABP of $68 \mathrm{mmHg}$ and below. As the oxygen buffers inside the blood gets depleted with decreasing $\mathrm{ABP}$, the $\mathrm{TiPO}_{2}$ decreases, until the point is reached, from which onwards there is not enough oxygen left to supply the metabolism of the brain tissue.

\subsubsection{Case 2: no swelling, autoregulation}

As for the graphs for enabled autoregulation, which can be seen in figure 8.2, the development of the $\mathrm{TiPO}_{2}$ has more or less a similar slope as the ICP and the corresponding CBF (compare fig. 6.7).

The difference between the development of the $\mathrm{CBF}$ and $\mathrm{TiPO}_{2}$ lies in the different development outside the autoregulation range. Above the upper autoregulation point, the increase in $\mathrm{TiPO}_{2}$ is smaller. Below the lower autoregulation point, the drop in $\mathrm{TiPO}_{2}$ is more rapidly than the drop of CBF, as the oxygen buffers start to become depleted. Furthermore, the decrease of $\mathrm{TiPO}_{2}$ has a concave like behavior, contrary to the convex like decrease of ICP and ABP.

\subsubsection{Case 3: swelling, no autoregulation}

Figure 8.3 shows the graphs for ICP and $\mathrm{TiPO}_{2}$ for the case, in which the autoregulation mechanism was disabled and a swelling was simulated.

For ABP values below $98 \mathrm{mmHg}$, both graphs have a similar run as in the previous simulation without a swelling and disabled autoregulation. As the CSF compartment is depleted above $98 \mathrm{mmHg}$, the ICP raises more 


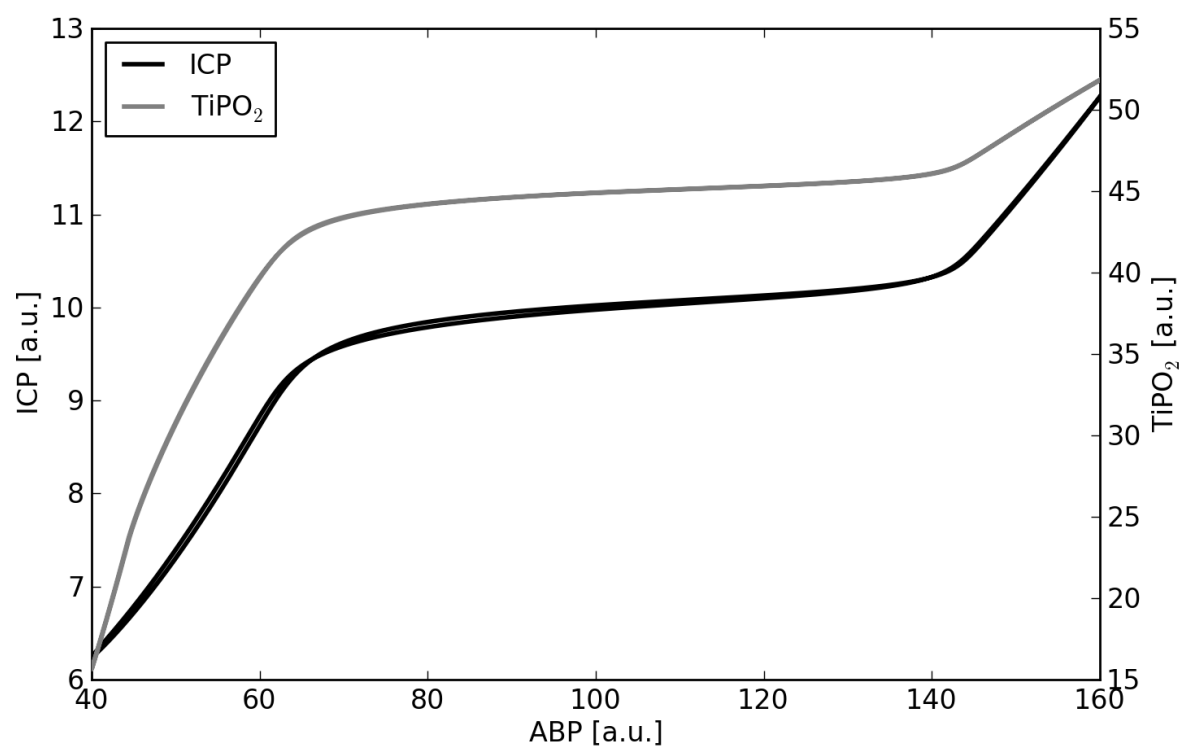

Figure 8.2: $\mathrm{TiPO}_{2}$ and ICP against ABP with $\alpha=1.4$ and $V_{E}=0 \mathrm{ml}$ (sHOM; case 2).

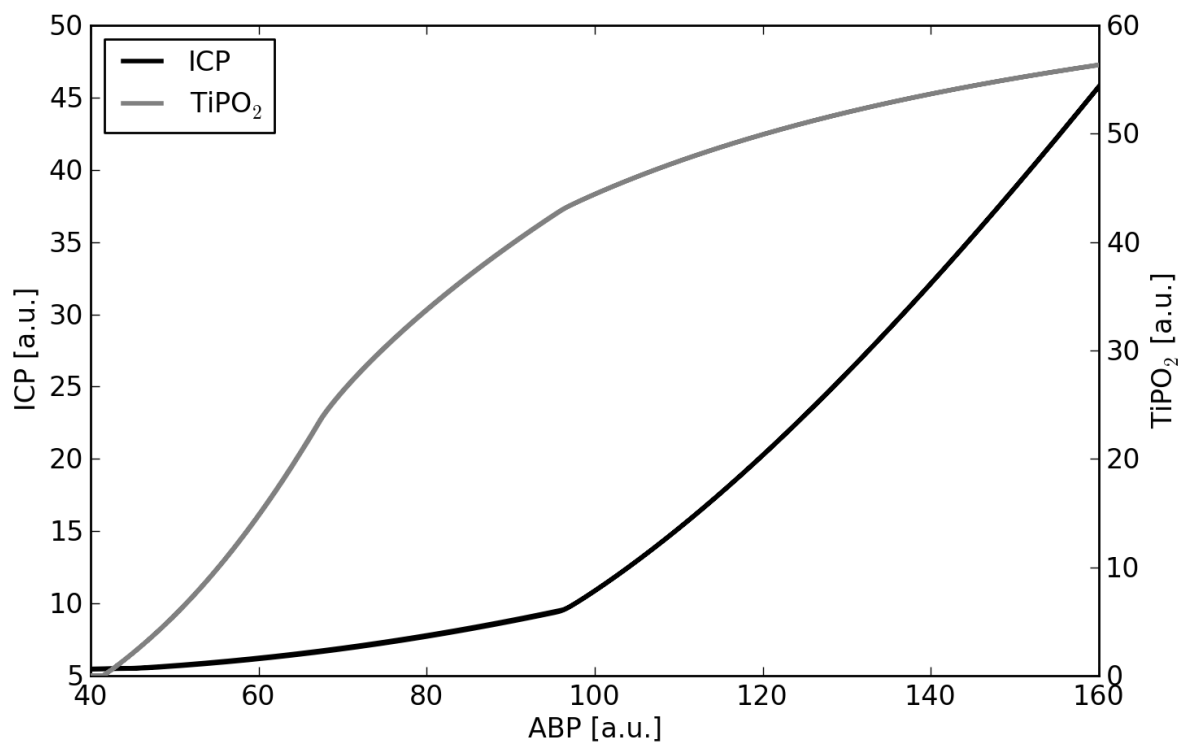

Figure 8.3: $\mathrm{TiPO}_{2}$ and ICP against ABP with $\alpha=0.0$ and $V_{E}=30 \mathrm{ml}$ (sHOM; case 3 ). 
rapidly than in the corresponding simulation. This results in a decrease in CBF, which ultimately results in a diminished increase of $\mathrm{TiPO}_{2}$, when compared to the simulation with no swelling (case 1 ). The maximal values for $\mathrm{TiPO}_{2}$ between both simulations differ by $10 \mathrm{mmHg}$.

\subsubsection{Case 4: swelling, autoregulation}

The last simulation for the starling model, in which the autoregulation is enabled and a swelling is simulated, is the most interesting one. As can be seen in figure 8.4, the drop in $\mathrm{CBF}$ between an $\mathrm{ABP}$ of $85 \mathrm{mmHg}$ and $50 \mathrm{mmHg}$ results in a drop in $\mathrm{TiPO}_{2}$, as expected.

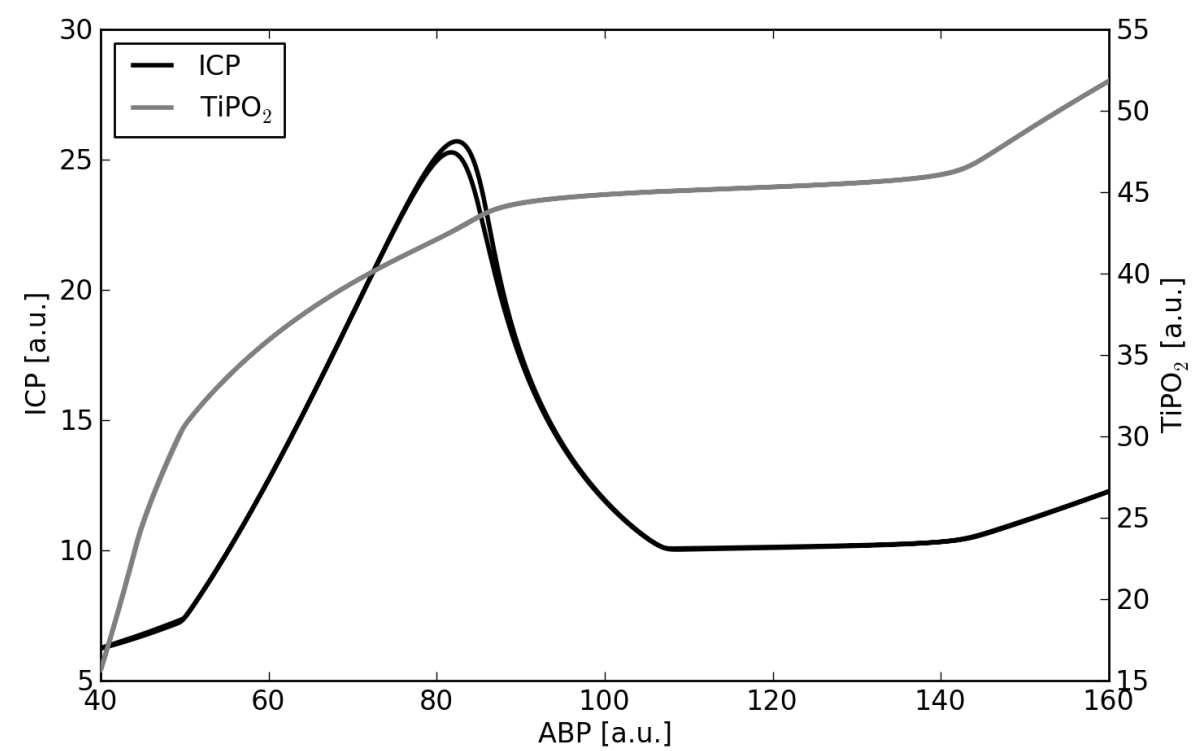

Figure 8.4: $\mathrm{TiPO}_{2}$ and ICP against ABP with $\alpha=1.4$ and $V_{E}=30 \mathrm{ml}$ (sHOM; case 4).

An interesting aspect is the small interval of negative correlation between $\mathrm{TiPO}_{2}$ and ICP. Here, for decreasing ABP below $105 \mathrm{mmHg}$, the partial oxygen pressure inside the tissue decreases. But due to the depleted CSF compartment, the ICP increases with decreasing ABP, which results in a negative coupling of ICP and $\mathrm{TiPO}_{2}$.

\section{2 esHOM model}

In this sections, the development of the partial oxygen pressure is discussed for the esHOM model. As the sHOM and the esHOM models produce 
equal results for the non-swelling scenario and identical autoregulation in each hemisphere, case 1 and 2 are omitted, as their results yield no new information. Besides case 3 and 4 , in addition the simulations for a mixed autoregulation are examined, as have been done in chapter 7 .

\subsubsection{Case 3: swelling, no autoregulation}

For disabled autoregulation and a swelling, there are only small differences between the corresponding simulations done with sHOM and esHOM. The graphs for esHOM are shown in figure 8.5.

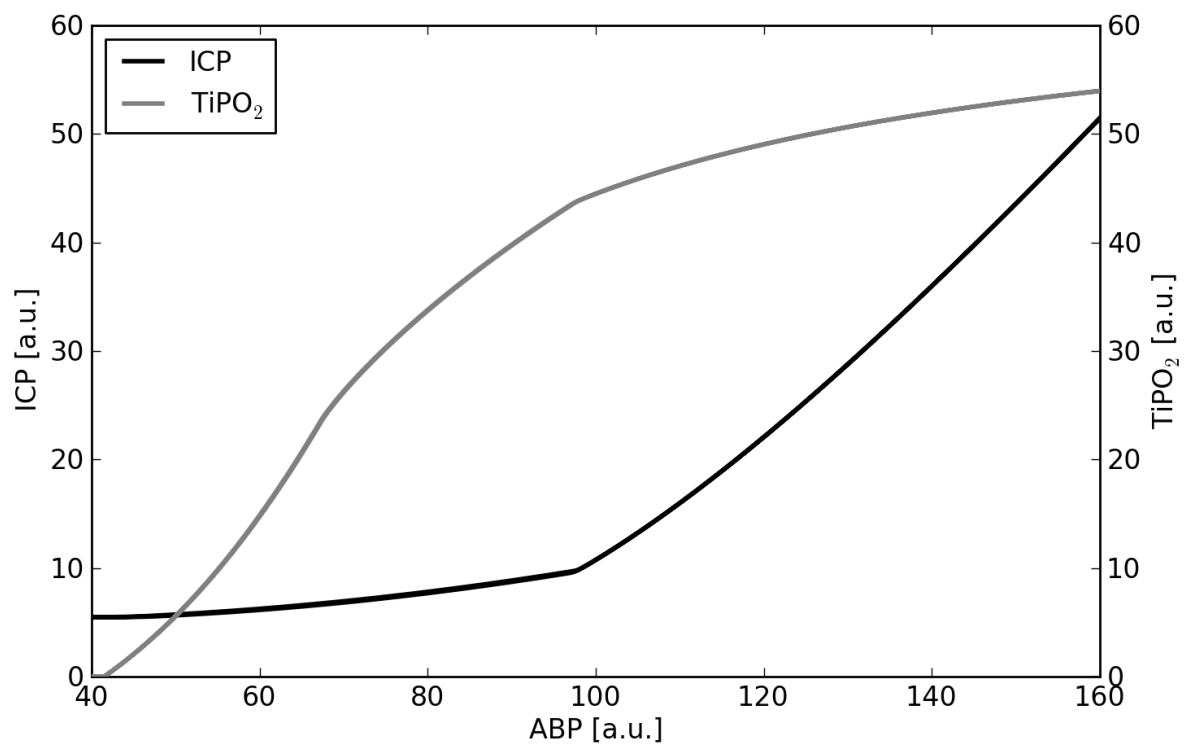

Figure 8.5: $\mathrm{TiPO}_{2}$ and ICP against $\mathrm{ABP}$ with $\alpha^{i}=0.0$ and $V_{E}=30 \mathrm{ml}$ (esHOM; case 3).

For $\mathrm{ABP}$ values below $98 \mathrm{mmHg}$, the obtained values in both scenarios are identical, as the CSF compartment is able to absorb the volume changes (when comparing fig. 8.5 with fig. 8.3, take note of the different $y$-axis for ICP in both graphs).

Due to its depletion and the different volume development of the blood vessels, the behavior differs above an $\mathrm{ABP}$ of $98 \mathrm{mmHg}$. This results in an increased ICP for the simulations with a swelling for the esHOM model. Due to the increased ICP, the CBF reaches smaller values and therefore, the $\mathrm{TiPO}_{2}$ is smaller and its maximal value for an $\mathrm{ABP}$ of $140 \mathrm{mmHg}$ is about $4 \mathrm{mmHg}$ below the value in the simulations with the sHOM model. 


\subsubsection{Case 4: swelling, autoregulation}

From the previous sections it can be assumed, that the simulations for a swelling and enabled autoregulation will contain only predictable results. The graphs for the $\mathrm{TiPO}_{2}$ and ICP are depicted in figure 8.6.

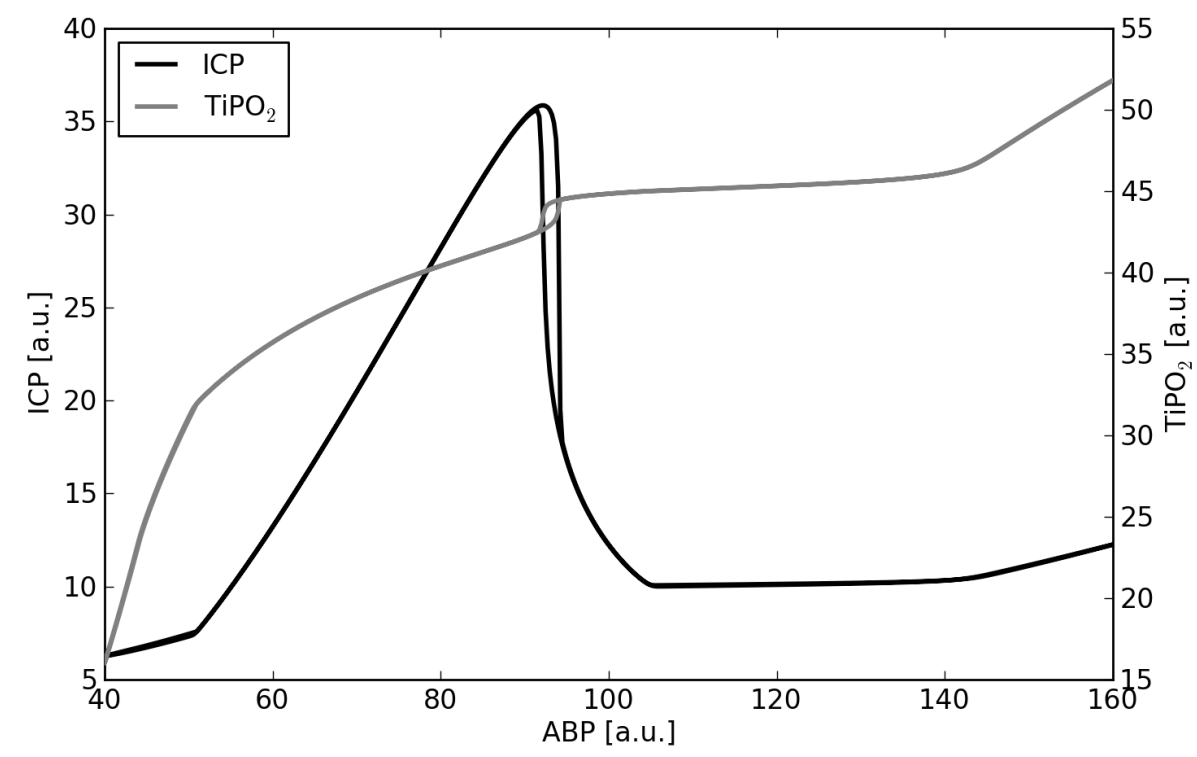

Figure 8.6: $\mathrm{TiPO}_{2}$ and ICP against ABP with $\alpha^{i}=1.4$ and $V_{E}=30 \mathrm{ml}$ (esHOM; case 4).

As in the corresponding simulations the drop, seen in the CBF, can also be seen in the $\mathrm{TiPO}_{2}$ around the maximum of the sharp peak in ICP, but the result looks unnatural and, with its occurrence, conflicts with medical experience. But it should be emphasized, that the simulations were done under assumptions, which may as such not or only rarely occur in reality.

Additionally it should be mentioned, that the maximum of the ICP can be adjusted by altering the constant $k_{V}$ and with it the compliance of the veins. A decrease of $k_{V}$ will result in a smaller maximum of ICP. But due to the different volume development of the veins, the drop in CBF between an $\mathrm{ABP}$ of 55 and $90 \mathrm{mmHg}$, and with it in $\mathrm{TiPO}_{2}$, is smaller.

As the esHOM model reflects the assembling of the brain more accurately, it should in general yield better results. But as already seen in the previous chapters, a one on one adoption of the used parameters, even if altered accordingly, as were the compliances and resistances, is no longer possible. Therefore, new values have to be found or new boundary conditions and restrictions for variables may be introduced. 


\subsubsection{Case 5: no swelling, mixed autoregulation}

Figure 8.7 shows the graphs for ICP and $\mathrm{TiPO}_{2}$ for the esHOM simulations with no swelling and a mixed autoregulation.

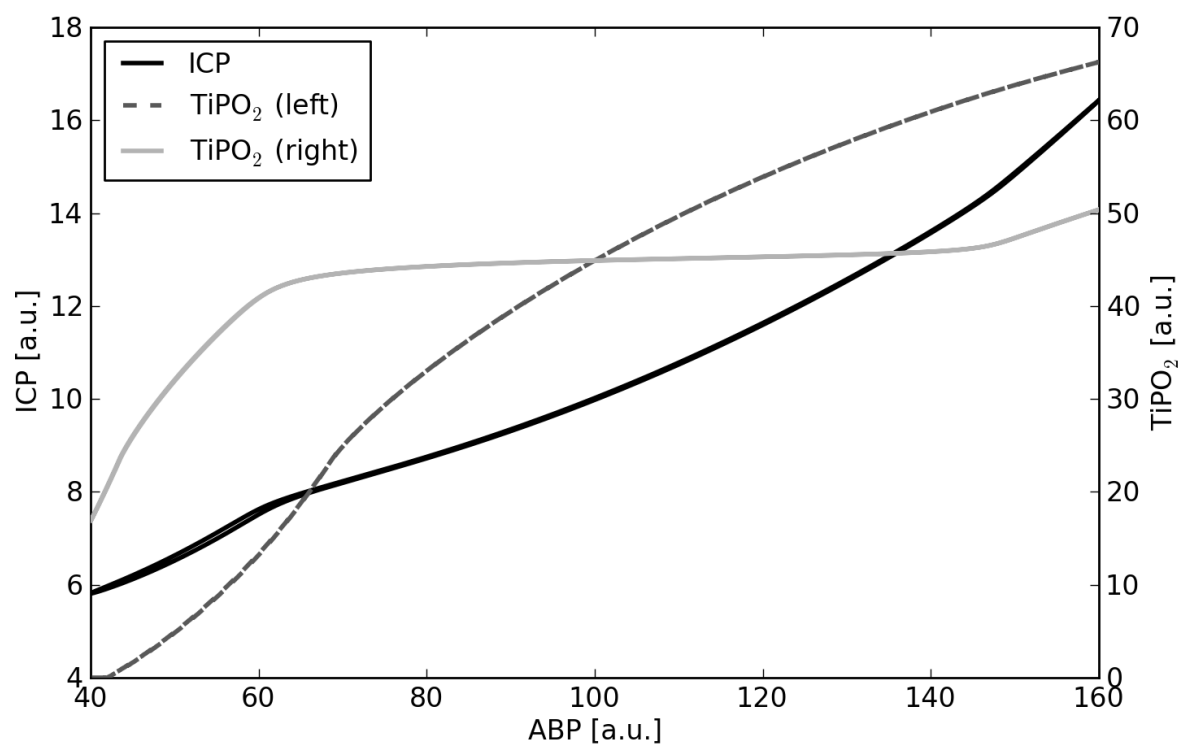

Figure 8.7: $\mathrm{TiPO}_{2}$ and ICP against ABP with $\alpha^{L}=0, \alpha^{R}=1.4$ and $V_{E}=0 \mathrm{ml}$ (esHOM; case 5).

From the previous sections and the discussion in section 7.4.1, the graphs for the $\mathrm{TiPO}_{2}$ in the left and right hemisphere could have been deduced. In principal, the values for the $\mathrm{TiPO}_{2}$ in each hemisphere are equal to the values obtained by the corresponding simulations with identical autoregulation, with some minor deviations.

The difference between case 1, 2 and 5 mainly lies in the different ICP development. Here, the run of the ICP has a more linear development, contrary to the concave development in case 1 and the plateau, as seen in the simulations done in case 2 .

\subsubsection{Case 6: swelling, mixed autoregulation}

As for the last case, in which the simulations were done with a swelling and mixed autoregulation, the results for the $\mathrm{TiPO}_{2}$ in each hemisphere and the associated ICP are depicted in figure 8.8.

For an $\mathrm{ABP}$ below $95 \mathrm{mmHg}$, the graphs for case 5 and 6 are equal, as the the volume changes inside the skull can be absorbed by the CSF compartment. As there is no CSF left to absorb for higher ABP values, the 


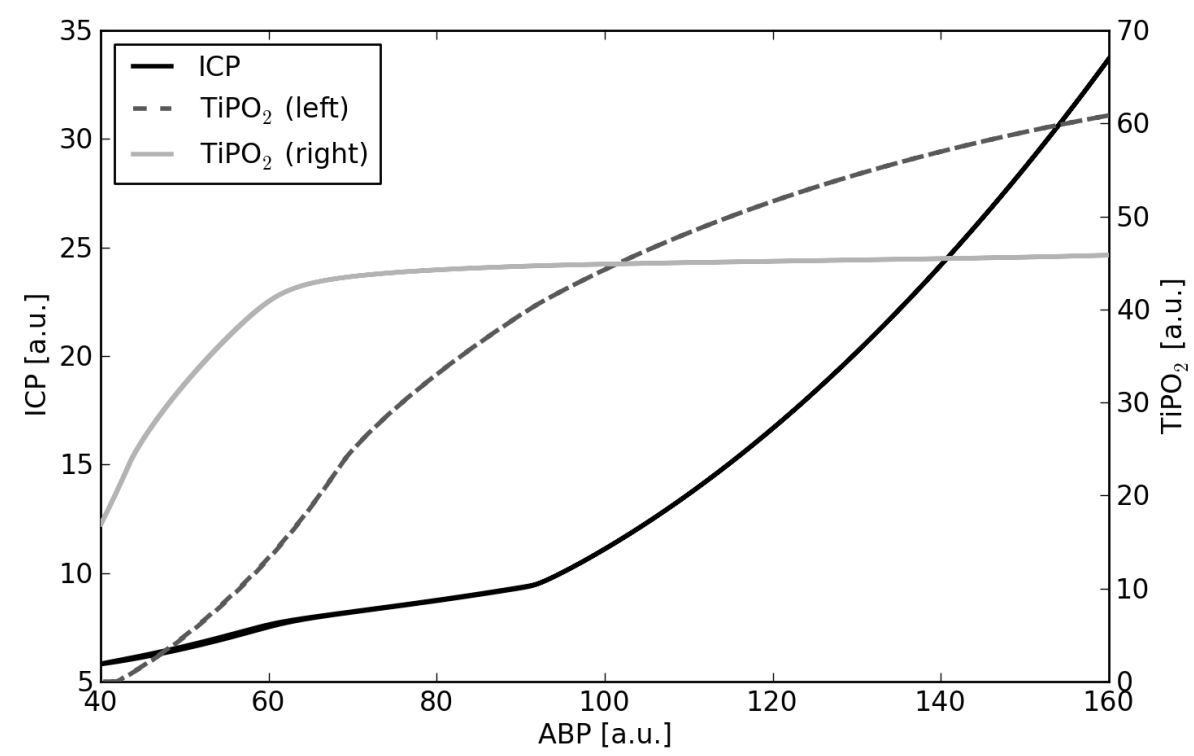

Figure 8.8: $\mathrm{TiPO}_{2}$ and ICP against $\mathrm{ABP}$ with $\alpha^{L}=0, \alpha^{R}=1.4$ and $V_{E}=30 \mathrm{ml}$ (esHOM; case 6).

ICP increases due to the net increase in volume of the blood vessels with increased ABP. This results in a reduced $\mathrm{TiPO}_{2}$ development in the left hemisphere, as there the increase of $\mathrm{CBF}$ grows slower in case 6 than in case 5.

In the right hemisphere, the autoregulation plateau is extended, which results in the situation, that the $\mathrm{TiPO}_{2}$ remains nearly constant, although the ICP increases. The reason for this development is the smaller difference between ABP and ICP, which results in a smaller constriction of the arteries, when compared e.g. to case 2 (also see section 7.4.2).

\subsection{Placement of the models on the basis of ex- perimental data}

The original intention of modeling the cerebral haemodynamics and the oxygen transport was, to gain a more detailed picture of the interaction between the various cerebral regulatory systems, which govern the CBF. Therefore, a valid model is supposed to be able to reproduce known behavior, but with it, it should also be possible to explain developments in measured data, which are not yet fully understood.

In the previous chapters it was shown, that in general all different models 
presented in this work are able to reproduce the effects seen in standard experiments, like the autoregulation curve, introduced in section 2.2.1. To be able to compare the quality of the models among each other, a special time section of measured data is used, in which $\mathrm{ABP}$ and $\mathrm{TiPO}_{2}$ are correlated, but each are anti-correlated with respect to ICP. In addition an attempt is made, to determine the underlying processes of this development from a model point of view, as the origin of this effect is not yet fully understood.

\subsubsection{Experimental data}

The measured data shown in the following, stems from a patient with severe head injury. It was recorded at the Department of Neurosurgery of the University Hospital Regensburg. In addition, the data has been preprocessed. Information on the preprocessing can be found in [5]. But primarily, the measured data is filtered with a low pass filter with a cutoff frequency of $0.002 \mathrm{~Hz}$, in order to remove oscillatory components like heartbeat and breathing.

In figure 8.9, a segment of these recordings is shown for an ABP development against time. In addition, the development of the corresponding partial oxygen pressure in tissue is depicted, too. From a qualitative point of view, the graphs for $\mathrm{ABP}$ and $\mathrm{TiPO}_{2}$ have more or less the same course. This type of behavior can be reproduced by all models, for the case, where $\alpha$ remains small or the autoregulation is completely disabled. There, with increasing ABP the partial oxygen pressure in tissue also rises. The difference between all cases and models only lies in the amount of the increase.

Figure 8.10 shows the run of the ABP and, in addition, the ICP development for the same time interval. But here, the development of the ICP is totally different, when compared to the ABP. For raising ABP the ICP decreases and vice versa. The same holds, when the ICP development is compared to the partial oxygen pressure in tissue, as the $\mathrm{ABP}$ and $\mathrm{TiPO}_{2}$ have a similar development, as can be seen in figure 8.11. Also, the opposed development of both measurements is more obvious.

\subsubsection{Comparison with modeled data}

In the following, the prerequisites will be determined, which are necessary to reproduce this scenario with the models. In addition, an explanation for this behavior from a model point of view will be given.

\section{Preliminary considerations}

The main characteristic of the measured data is an increasing ICP with decreasing ABP. Due to the in-depth examinations done in the previous chapters for all introduced variations of the model, the following conditions 


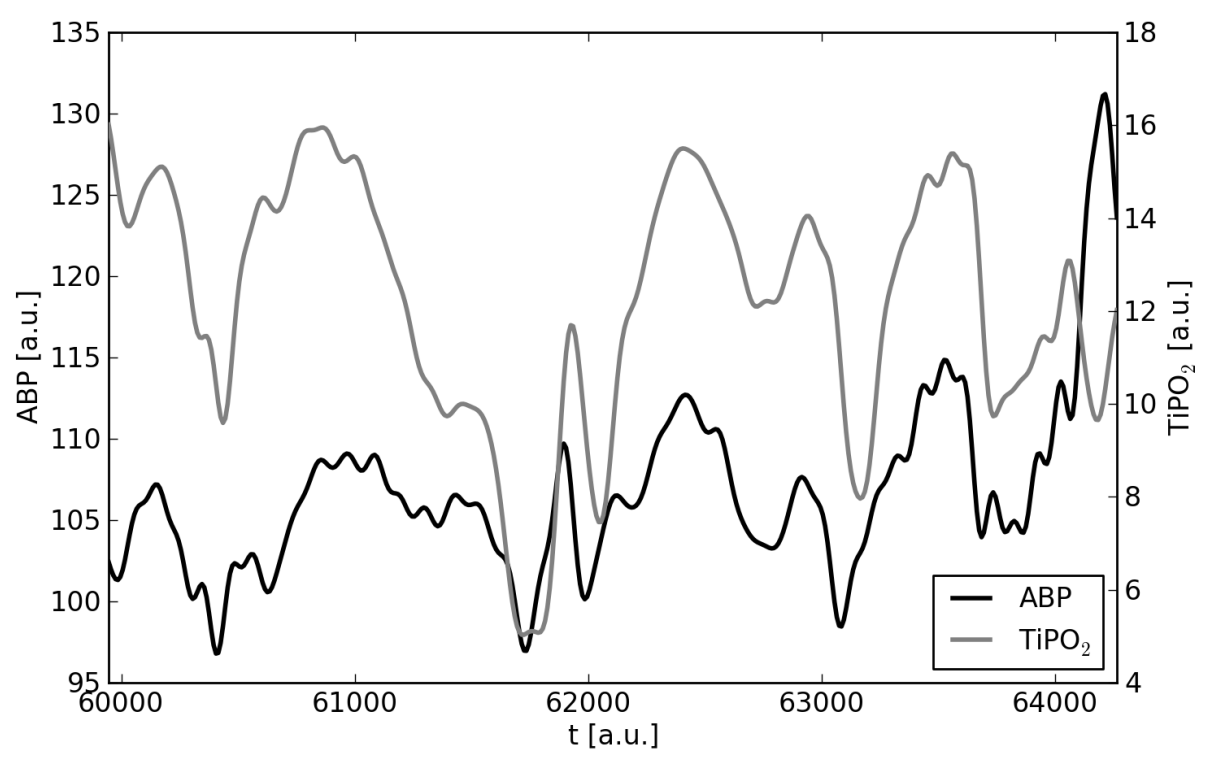

Figure 8.9: Measured data; $\mathrm{ABP}$ and $\mathrm{TiPO}_{2}$ against time.

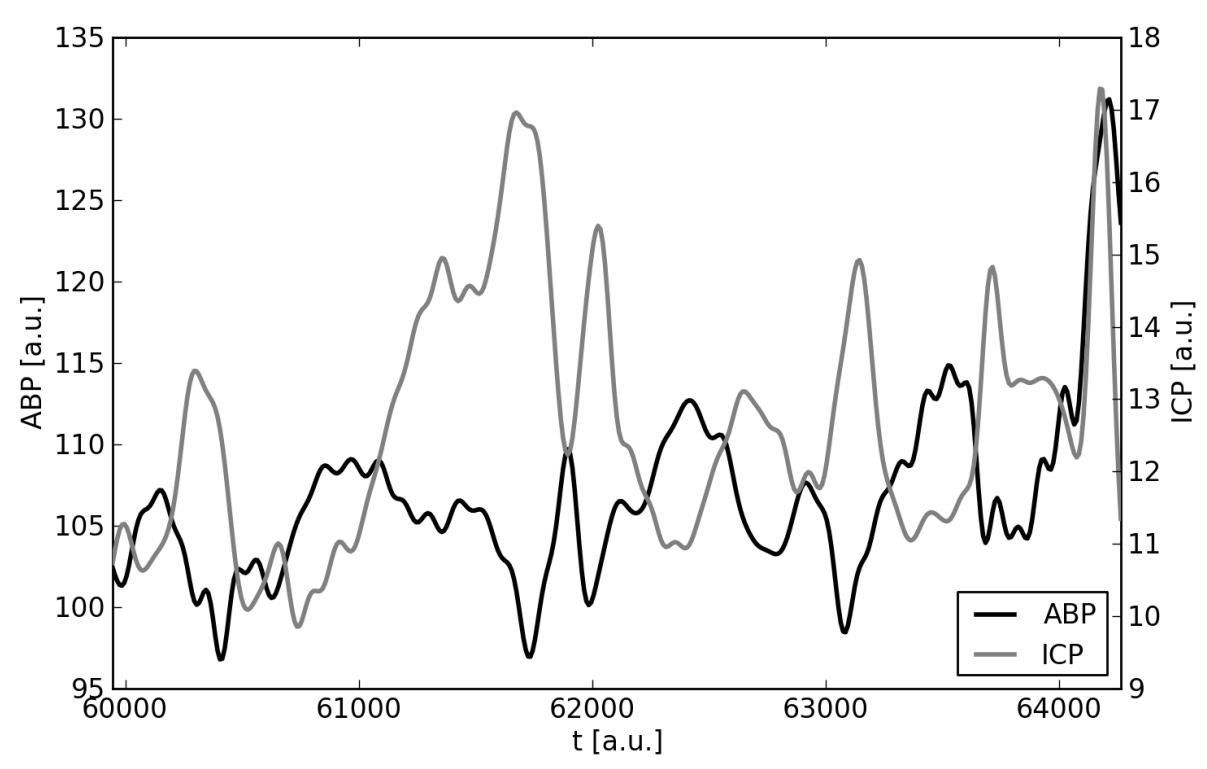

Figure 8.10: Measured data; ABP and ICP against time. 


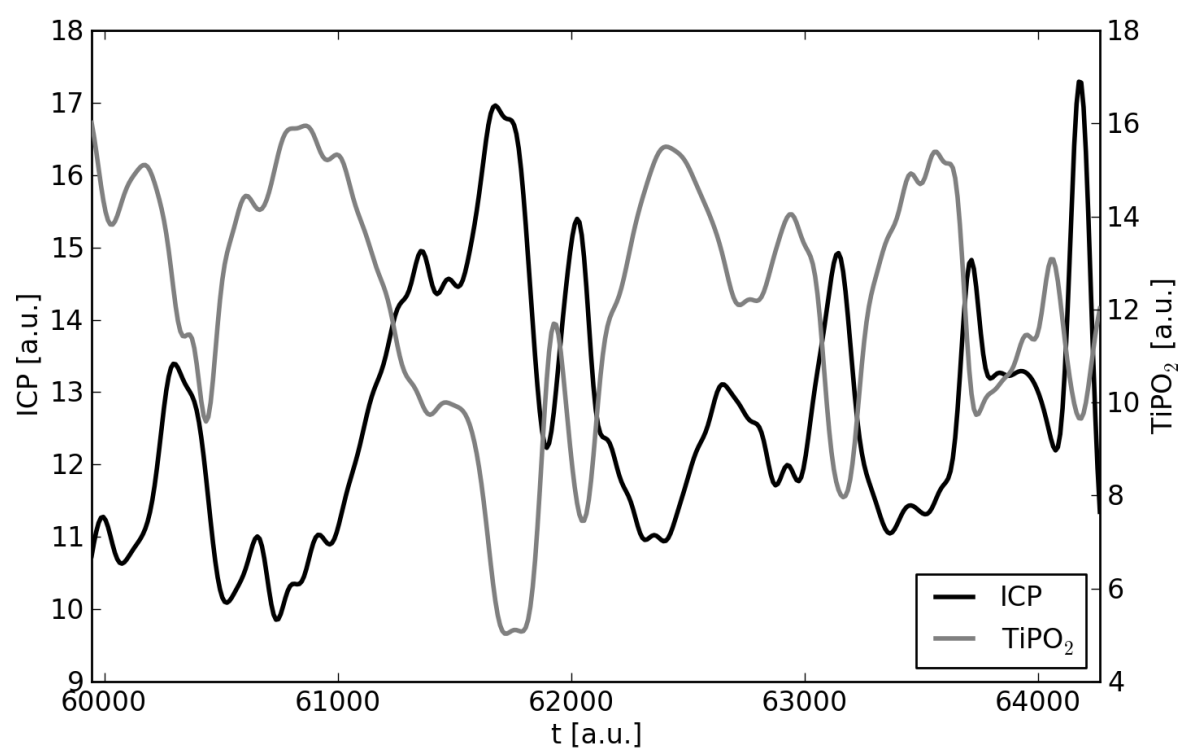

Figure 8.11: Measured data; ICP and $\mathrm{TiPO}_{2}$ against time.

can be extracted, with which it is possible to simulate this behavior in general:

- a swelling exists

- the autoregulation mechanism is not disabled

- the autoregulation mechanism is somehow restricted (compare fig. 4.3)

A swelling is needed, in order to create an interval, in which no CSF is left to absorb. In this interval, the ICP will have increased values, when compared to the no swelling case (compare case 1 and 3 or case 2 and 4, respectively; true for all variations of the model).

To achieve an increase in ICP with decreasing ABP, the total volume of the blood vessels has to increase. As the ABP is decreasing, this is only possible, if the autoregulation mechanism is not disabled. For an ABP below the equilibrium point of the autoregulation mechanism, the arteries then will compensate the decreasing $\mathrm{ABP}$ by increasing their diameter. If this happens inside an ABP range, in which there is no CSF left to absorb, this will result in an increasing ICP for decreasing ABP, as long as the lower autoregulation point is not reached.

In figure 8.9 it can be seen, that the partial oxygen pressure has a noticeable variation. With the previous deduction, the autoregulation mechanism 
has to work in a restricted way, in order to increase the total volume of the blood vessels, but also to allow a variation in $\mathrm{TiPO}_{2}$. Also, to reproduce the measured $\mathrm{TiPO}_{2}$ values, the parameters for the oxygen transport have to be adjusted. When directly comparing the simulated $\mathrm{TiPO}_{2}$ values of the previous simulations in this chapter with the measured values in this section, the simulated values are too high.

From the previous chapters it can also be deduced, that the bigger the simulated swelling, the bigger the interval, in which the CSF compartment is depleted. In order to have an increasing ICP with decreasing ABP above $100 \mathrm{mmHg}$, the swelling must be at least of the order of the absorbable CSF volume.

\section{Conditions for simulation}

From the preliminary considerations the following conditions for the simulations of the CBF must be met:

- $0<\alpha<2$

- $V_{E}>30 \mathrm{ml}$

As for the oxygen transport, the parameters have to be altered in such a way, that the partial oxygen pressure is reduced, when compared to the deduced standard values.

With these boundary conditions, there is a broad band of parameters, which fulfill the assumed requirements. Due to the focus of this work, there is no algorithm implemented yet, to determine the optimal set of parameters, which reproduces the measured data best.

The parameters $\alpha$ and $V_{E}$ were chosen in such a way, that the ICP and $\mathrm{TiPO}_{2}$ of the simulations show a desired behavior. To reduce the $\mathrm{TiPO}_{2}$, only the oxygen consumption rate was adjusted. This still allows a qualitative and quantitative comparison between the simulated and measured experiment. The simulations itself were done with the following adjusted set of parameters:

$$
\begin{aligned}
\alpha^{(i)} & =0.5 \\
V_{E} & =31 \mathrm{ml} \\
A & =13.5 \cdot 10^{-4} \frac{{\mu \mathrm{m}^{3} \mathrm{O}_{2}}_{\mathrm{m}^{3} \cdot \mathrm{s}}}{\mathrm{u}^{2}}
\end{aligned}
$$

For comparison, here the standard values for $V_{E}$ and $A$ in the previous simulations are shown:

$$
\begin{aligned}
V_{E} & =30 \mathrm{ml} \\
A & =4.5 \cdot 10^{-4} \frac{\mathrm{\mu m}^{3} \mathrm{O}_{2}}{\mu^{3} \cdot s}
\end{aligned}
$$


The ABP interval of interest lies between an ABP of approx. 96 and $116 \mathrm{mmHg}$. With it, the range of the ICP is about $7 \mathrm{mmHg}$. For the $\mathrm{TiPO}_{2}$, the range is approx. $11 \mathrm{mmHg}$. Therefore, the simulations will be done for an ABP between 80 and $120 \mathrm{mmHg}$, in order to obtain the whole interval of interest.

\section{Simulations with bHOM model}

Figure 8.12 shows the results of the simulation for the (e)bHOM models with and without separated hemispheres. A prepended "b" denotes the basic model for one hemisphere and a prepended "e" the basic model with two hemispheres.* As the parameters for the esHOM model were chosen symmetrically, the results of only one hemisphere are shown.

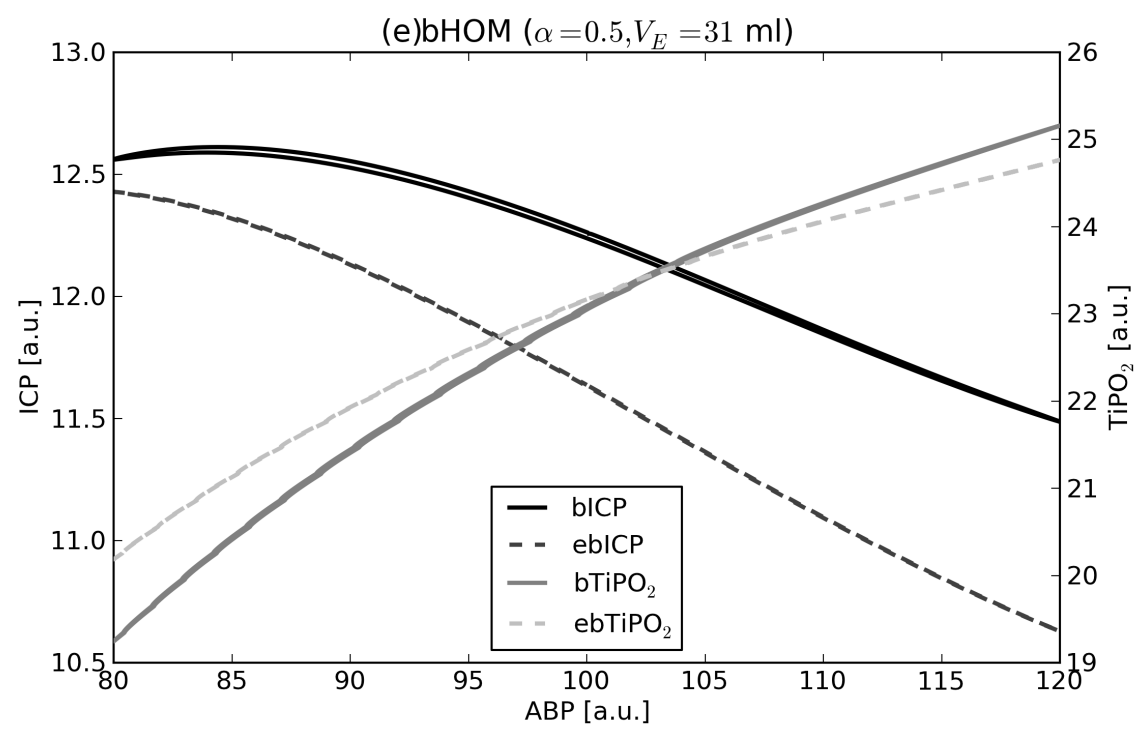

Figure 8.12: Simulation: ICP and $\mathrm{TiPO}_{2}$ against $\mathrm{ABP}$ for (e)bHOM models (with ("eb") and without ("b") separated hemispheres) for $\alpha^{(i)}=0.5$ and $V_{E}=31 \mathrm{ml}$.

Although the graphs show a similar behavior for $\mathrm{TiPO}_{2}$ in both simulations, as seen in fig. 8.9, the obtained variations of approx. $6 \mathrm{mmHg}$ are about a factor of two too small. The obtained ranges for ICP, with a variation smaller than $2 \mathrm{mmHg}$, are outside the needed scope. It is save to say, that the simulations are not able to reproduce the desired behavior, even from a qualitative point of view.

${ }^{*}$ Although the simulations with the ebHOM model were not discussed in the previous chapters, here, its results are shown for completeness. 
Therefore, the basic and extended basic model are not sufficient to simulate this type of measured data and must be altered.

\section{Simulations with (e)sHOM models}

The results for the sHOM and esHOM models are shown in fig. 8.13. To distinguish both simulations, as before, each simulated parameter is prepended with a "s" for the sHOM model and an "e" for the esHOM model with two hemispheres. As the autoregulation parameters in both hemispheres are identical, only the graphs for one hemisphere are shown.

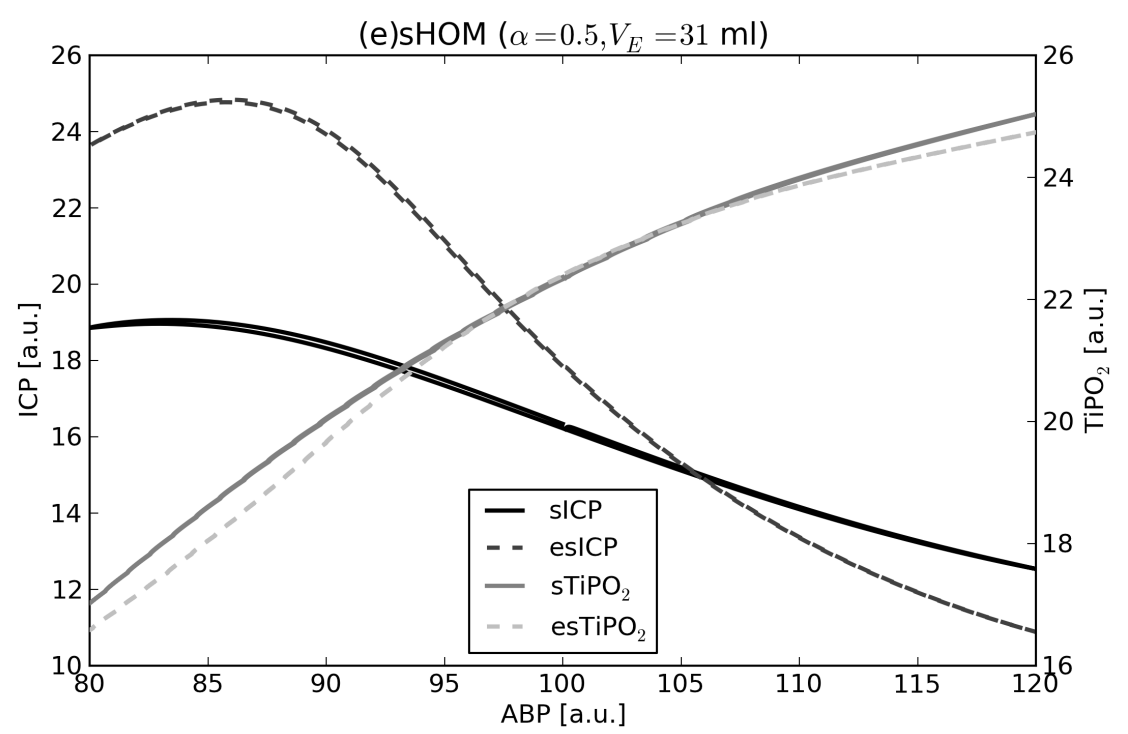

Figure 8.13: Simulation: ICP and $\mathrm{TiPO}_{2}$ against ABP for (e)sHOM model (with ("es") and without ("s") separated hemispheres) for $\alpha^{i}=0.5$ and $V_{E}=31 \mathrm{ml}$.

From a qualitative point of view, both simulations reproduce the measured dataset. For decreasing ABP below $120 \mathrm{mmHg}$, the partial oxygen pressure in tissue drops, whereas the ICP increases.

But from a quantitative point of view, the simulations with the esHOM model yield better results than the simulations done with the sHOM model. The interval for the ICP is much smaller in case of the sHOM model, than the corresponding one of the measured data. But the obtained values for ICP are still much better than for the basic model with and without two hemispheres.

On the contrary, the ranges of $\mathrm{ICP}$ and $\mathrm{TiPO}_{2}$ in the esHOM simulations are comparable to their corresponding measured values, even from a quantitative point of view. The crucial difference between sHOM and esHOM 
is the fact, that the blood vessel system in the esHOM simulations needs more space for a not disabled autoregulation than for the sHOM simulations, which results in an increased ICP.

The interaction between the volume of the swelling and the strength of the autoregulation and therefore the volume of the blood vessel system inside an ABP interval, in which the CSF compartment is depleted, determines the range of $\mathrm{TiPO}_{2}$ and ICP: In general with increasing swelling, the interval, in which the ICP is increased, broadens. With increasing strength of the autoregulation, the ICP raises, but the range of the $\mathrm{TiPO}_{2}$ is reduced. Both parameters have to be balanced in such a way, that their interaction results in the desired behavior.

However, with different parameters for the autoregulation mechanism in each hemisphere, the results can be further improved (compare fig. 8.14): With a fully functional autoregulation in the right $\left(\alpha^{R}=1.4\right)$ and a restricted autoregulation in the left $\left(\alpha^{L}=0.3\right)$ hemisphere, the CBF in the left hemisphere has a comparable range, whereas the ICP would increase for a $\mathrm{CBF}$ below $300 \mathrm{ml} / \mathrm{min}$, due to the increased volume of the blood vessel system. On the other hand, the range of the ICP could be maintained, but the range of the $\mathrm{TiPO}_{2}$ increased by using a smaller $\alpha$.

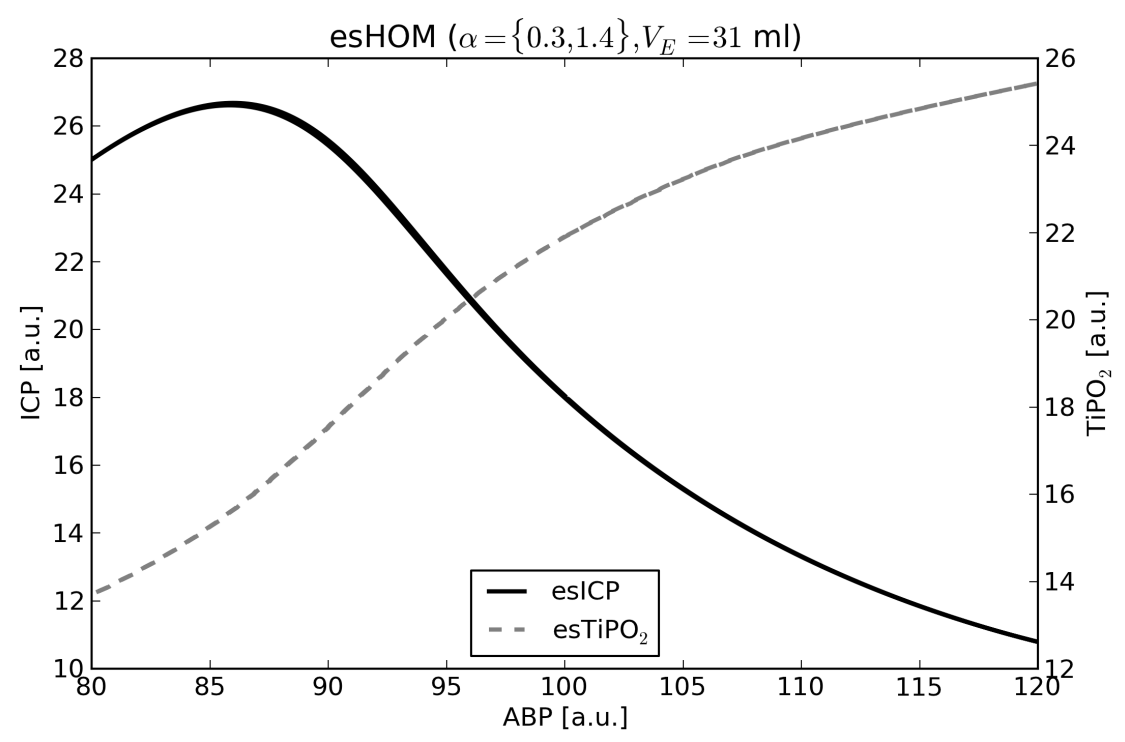

Figure 8.14: Simulation: ICP and $\mathrm{TiPO}_{2}$ against ABP for esHOM model, with mixed autoregulation, where $\alpha^{L}=0.3, \alpha^{R}=1.4$ and $V_{E}=31 \mathrm{ml}$. 


\subsubsection{Conclusion}

From this section, the following can be summarized:

- The basic models are not able to fully reproduce the haemodynamical processes of the brain.

As shown in this chapter, the basic models are not able to reproduce time intervals in measured data, in which $\mathrm{ABP}$ and $\mathrm{TiPO}_{2}$ are correlated and $\mathrm{ABP}$ and ICP are anti-correlated, which occur in more than $65 \%$ of patient specific measurements [5]. As this is a significant amount, this kind of behavior cannot be neglected and has to be assumed to be a relevant occurrence for measurements under the conditions, mentioned in the beginning of this section.

- To reproduce the aforementioned behavior of haemodynamical processes of the brain, the venous system has to be at least modeled with a starling resistor.

In order to achieve the increase of ICP with decreasing ABP, the starling resistor plays a crucial role: Without it, the increase of ICP, under the previously discussed circumstances, is too weak to explain the seen behavior. Therefore, the incorporation of the starling resistor into the model is essential.

- The best results can be achieved, when in addition to the starling resistor, a model is used, which separates the brain into two hemispheres.

With the sHOM, the behavior of the measured data can be explained and simulated in a qualitative way. But to simulate this behavior even in a quantitative way, the esHOM has to be used. Even in the case with a symmetrical autoregulation, the esHOM achieves better results, which can be further improved by using an asymmetrical autoregulation for each hemisphere. In addition, as the esHOM reflects natural circumstances of the human brain better, a wider range of simulations can be done, in which the state of the brain differs between the left and right hemisphere, like a disabled CSF production or a collapsed venous system in one hemisphere. Even simulating an asymmetrical blood supply in each hemisphere is possible with no additional changes to the model itself. 


\section{Chapter 9}

\section{Summary and outlook}

In this work, a series of models to simulate the haemodynamical processes and oxygen transport of the human brain have been introduced. After a short description of the interesting anatomical properties, a basic model, based on a compartmental approach, was derived, which incorporates these anatomical properties, in order to simulate the haemodynamical processes and the oxygen transport of the human brain. In addition to the original model, which was developed by Andreas Jung in cooperation with the Department of Neurosurgery of the University Hospital Regensburg, two extensions were proposed. On the one hand, for modeling the resistance of the veins a starling resistor was used. On the other hand, additionally, the model was split into two hemispheres, representing the left and right part of the human brain.

In the subsequent chapter, a detailed numerical analysis of the behavior of the basic model was done with respect to four different cases: A dis- and enabled autoregulation mechanism in combination with and without a cerebral swelling. For each above mentioned case, the inter-correlations of the underlying physiological processes or their absence were examined extensively. Therefore, non- or not easily measurable values like the volume development of each compartment and pressures, compliances and resistances of each compartment were discussed in detail. In particular, the influence of the autoregulation mechanism was shown, as was the influence of a swelling on the system. From a model point of view, the volume development of each compartment plays a crucial role, especially, when the cerebrospinal fluid (CSF) compartment is depleted. In this case, the behavior of the system differs significantly, as the volume changes of the blood vessels and brain tissue can no longer be compensated by the CSF itself. Another important results is, that the model reproduces experimentally well known behavior, like the autoregulation curve and the pressure volume curve.

To see the differences between the original model and the proposed extensions, first, the same type of simulations were redone in chapter 6 with 
the sHOM model, which is incorporating a starling resistor to describe the venous compartment. For the scenarios without a swelling, only small differences between both models were found. But for the scenarios with a swelling, the effects of the starling resistor became quite obvious. There, due to the increased volume change of the venous compartment, the intracranial pressure (ICP) reached increased values inside the arterial blood pressure (ABP) range, in which the CSF compartment was depleted. The increased volume change could no longer be compensated and therefore resulted in a more compressed brain tissue, which ultimately resulted in an increase in ICP.

Afterwards, the simulations were redone with both extensions, namely, including the starling resistor and the splitting of the brain into two hemispheres, in chapter 7. Similar to the sHOM model, the simulations with the esHOM model for identical autoregulation in both hemispheres and no swelling yielded a similar CBF. The main difference between both models is the increased volume change of the blood vessels, as the sum of the volume change of both arteries and veins has increased. For the scenarios with identical autoregulation in both hemispheres and a swelling, this increase in volume change was more dominant, due to the swelling. The CBF in both cases dropped significantly. In the scenario with enabled autoregulation and a simulated swelling the ICP rose dramatically, due to the autoregulation mechanism in addition to the swelling. The reason for this was the response of the arteries to the drop in CBF, caused by the decreasing ABP. Because of the dilation of the arteries, in order to stabilize the CBF, the ICP increased. With the increase in ICP, the pressure in the veins increased and with it, the pressure inside the capillaries. This resulted ultimately in the drop in CBF, which was larger for the esHOM model, than seen in the sHOM model.

In addition to the four scenarios, two new simulations with and without a swelling were done, in which the autoregulation was disabled in one hemisphere and enabled in the other. For the no swelling scenario, the coupling between both hemisphere was minimal and could be described as a small adjustment of the autoregulation in one hemisphere towards the autoregulation in the other hemisphere. In the scenario with a swelling, the coupling between both hemispheres was much higher in the ABP range, in which the CSF compartment was depleted. Due to a different ICP and volume development, the $\mathrm{CBF}$ in the hemisphere with disabled autoregulation was increased for $\mathrm{ABP}$ values inside this segment. The autoregulation plateau, as seen for the $\mathrm{CBF}$, was elongated in the other hemisphere, when compared to the scenario with an enabled and identical autoregulation in both hemispheres. This was due to the increased ICP, as the necessary regulations of the arteries were smaller, in order to maintain a constant CBF.

In the last chapter, a closer look was taken on the interplay of ABP, ICP and partial oxygen pressure in tissue $\left(\mathrm{TiPO}_{2}\right)$, as these quantities represent those values, which are measurable by physicians. In order to evaluate the quality of the models, each model was used to reproduce an interval of real 
world measured data of patients with a severe head injury. Inside these time segments, the ABP and $\mathrm{TiPO}_{2}$ are correlated, whereas the ABP and ICP are anti-correlated and therefore, ICP and $\mathrm{TiPO}_{2}$, too. The consequence of this type of correlation is, that the ICP raises, although the ABP decreases, which may be counterintuitive. The cause of the behavior seen in this type of data is not fully understood yet, but will occur in more than $65 \%$ of the patient specific data sets, which is a significant amount and therefore not unusual for the corresponding circumstances.

It could be shown, that the original model, on which all proposed extensions were based on, was not able to reproduce this behavior. For a qualitative reproduction of this effect, at least the venous system has to be modeled with a starling resistor. The fitting between experiment and simulation was further improved by using a separation of the brain into two hemispheres, which reflects the assembly of the human brain.

The explanation from a model point of view lies in the interplay between the brain tissue and volume development of the blood vessel system. Due to the assumed swelling, the CSF compartment is completely depleted. As the autoregulation mechanism is slightly restricted, but not disabled, the volume of the blood vessel system increases, in order to compensate the decrease of the ABP. This increase in volume of the blood vessel system cannot be compensated by the depleted CSF compartment and ultimately results in the increase of ICP with decreasing ABP.

In this work, the primary focus was laid on the aforementioned four particularly important states of the human brain. A next step will be, to use the model to reproduce a whole set of recorded data and interpret it. Therefore, an automated parameter fitting algorithm will have to be developed, to customize the parameter set to the monitored patient. This could be done by using a small interval in the recorded data with an appropriate development as a starting point. The idea is to create a tool, which is able to automatically determine the state of the haemodynamical processes. This tool should then analyze, if the autoregulation mechanism is working properly, and the state of the CSF compartment and with it, the magnitude of a swelling. Also, a forecast of trends, whether the state of a patient will improve or degrade, should be possible. This tool could then be used, to assist physicians in the interpretation of the monitored data and the physiological state of a patient.

Most of this work was concerned with a detailed understanding of the haemodynamical processes inside the human brain. The second part, namely the oxygen transport, played a minor (but necessary) role. Another step will be, to improve the understanding of the oxygen transport itself, in order to better address the shortcomings, seen in chapter 8 .

In general, a detailed understanding of the underlying processes is gained from a model point of view and therefore, it will be much easier to further adjust this compartmental approach, when deemed necessary. 


\section{Bibliography}

[1] H. An, W. Lin, A. Celik and Y. Z. Lee: Quantitative measurements of cerebral metabolic rate of oxygen utilization using MRI: a volunteer study, NMR in Biomedicine, 14:441-447, (2001)

[2] W. Bargmann: Histologie und mikroskopische Anatomie des Menschen, Georg Thieme Verlag, 7th edition, (1977)

[3] S. Cirovic, C. Walsh, W.D. Fraser: Mathematical study of the role of non-linear venous compliance in the cranial volume-pressure test, Med. Biol. Eng. Comput., 41, 579-588, (2003)

[4] S. Cunningham, S. Deere, A. Symon, R.A. Elton, N. McIntosh: A randomized, controlled trial of computerized physiological trend monitoring in an intensive car unit, Crit Care Med., 26(12) 2053-2060, (1998)

[5] R. Faltermeier, M.A. Proescholdt, and A. Brawanski: Computerized Data Analysis of Neuromonitoring Parameters Identifies Patients with Reduced Cerebral Compliance as Seen on CT, Intracranial Pressure and Brain Monitoring XIV, Acta Neurochirurgica Supplementum, Supplement 114, in print, (2012)

[6] H. Gray: Anatomy of the Human Body, taken from wikipedia, Lea \& Febiger, Philadelphia, (1918)

[7] M.S. Greenburg: Handbook of Neurosurgery, Greenburg Graphics Inc., (1991)

[8] R. Greger and U. Windhorst: Comprehensive Human Physiology, volume 2, chapter "Oxygen supply and energy metabolism", Springer Verlag, (1996)

[9] A.V. Hill: The possible effects of the aggregation of the molecules of hemoglobin on its dissociation curves, Journal of Physiology, XL:iv-vii, (1910)

[10] A. Jung: A mathematical model of the hydrodynamical processes in the brain - a rigorous approach, Proceedings of Workshop GK "Nonlinearity" - Regensburg, (2002) 
[11] A. Jung: Statistical analysis of biomedical data, PhD Thesis, (2003)

[12] G.I. Mchedlishvili: Arterial behaviour and blood circulation in the brain, Plenum Press, New York, (1986)

[13] N. McIntosh, A.J. Lyon, J. Reiss et al.: The cognitive process of doctors and nurses in the interpretation of physiological monitoring data in the neonate, Early Hum Dev, 58: 73, (2000)

[14] M.A. Mintun, B.N. Lundstrom, A.Z. Snyder, A.G. Vlassenko, G.L. Shulman and M.E. Raichle: Blood flow and oxygen delivery to human brain during functional activity: Theoretical modeling and experimental data, Proceedings of the National Academy of Sciences, 98(12):6859-6864, (2001)

[15] F.H. Netter: Farbatlanten der Medizin, The Ciba Collection of Medical Illustrations, Band 5, Nervensystem I, Georg Thieme Verlag Stuttgart, New York, (1987)

[16] M.J. Purves: The Physiology of the Cerebral Circulation, Cambridge University Press, (1972)

[17] U. Raff, A.L. Scherzinger, P.F. Vargas, and J.H. Simon: Quantitation of grey matter, white matter, and cerebrospinal fluid from spin-echo magnetic resonance images using artificial neural network techniques, Medical Physics, 21(12):1933-1942, (1994)

[18] D.D. Reneau, D.F. Bruley and M.H. Knisely: Chemical Engineering in Medicine and Biology, chapter "A mathematical simulation of oxygen release, diffusion, and consumption in the capillaries and tissue of the human brain", Plenum Press, New York, pages 135-241, (1967)

[19] D.D. Reneau, D.F. Bruley and M.H. Knisely: A digital simulation of transient oxygen transport in capillary-tissue systems (cerebral grey matter), AIChE Journal, 15(6):916-925, (1969)

[20] F.J.W. Roughton and J.W. Severinghaus: Accurate determination of $\mathrm{O}_{2}$ dissociation curve of human blood above $98.7 \%$ saturation with data on $\mathrm{O}_{2}$ solubility in unmodified human blood from $0^{\circ}$ to $37^{\circ} \mathrm{C}$, Journal of Applied Physiology, 35(6):861-869, December (1973)

[21] J. Sahuquillo: Does multimodality monitoring make a difference in neurocritical care?, European Journal of Anaesthesiology, 25 (Suppl 42): 83-86, (2008)

[22] R.F. Schmidt, G. Thews, and F. Lang: Physiologie des Menschen, Springer Verlag, 28th edition, (2000) 
[23] J.W. Severinghaus: Simple, accurate equations of human blood $\mathrm{O}_{2}$ dissociation computations, Journal of Applied Physiology, 46:599-602, January-June (1979)

[24] M.R. Shah, V. Hasselblad, L.W. Stevenson et al.: Impact of the pulmonary artery catheter in critically ill patients: meta-analysis of randomized clinical trials, JAMA, 294: 1664-1670, (2005)

[25] M. Sharan and A.S. Popel: A compartment model for oxygen transport in brain microcirculation in the presence of blood substitutes, Journal of theoretical Biology, 216:479-500, (2002)

[26] H.G. Sullivan and J.D. Allison: Physiology of cerebrospinal fluid, Neurosurgery, volume 3, pages 2125-2135, (1985)

[27] G.A. Truskey, F. Yuan, and D.F. Katz: Transport Phenomena in Biological Systems, chapter "Oxygen-Hemoglobin Equilibria", Prentice Hall, (2004)

[28] M. Ursino: A mathematical study of human intracranial hydrodynamics: Part 1 - The cerebrospinal fluid pulse pressure, Annals of Biomedical Engineering, 16:379-401, (1988)

[29] M. Ursino and C. Lodi: A simple mathematical model of the interaction between intracranial pressure and cerebral haemodynamics, Journal of Applied Physiology, 82(4):1256-1269, (1997)

[30] M. Ursino and C.A. Lodi: Interaction among autoregulation, $\mathrm{CO}_{2} \mathrm{re}$ activity, and intracranial pressure: a mathematical model, American Physiological Society, (1998)

[31] Wikipedia, http://en.wikipedia.org/wiki/File:Circle_of_Willis_en.svg, (08.12.2009)

[32] C. Woertgen, R. Faltermeier, R. Rothörl and A. Brawanski: Vergleich von ventrikelnahen und kortikalen intracraniellen Drücken, Jahrestagung der Sektion Intracranieller Druck, Hirndurchblutung, Hirnödem der Deutschen Gesellschaft für Neurochirugie, Berlin, (2003)

[33] A. Zwart, G. Kwant, B. Oeseburg and W.G. Zijlstra: Human wholebody oxygen affinity: effect of temperature, Journal of Applied Physiology, 57:429-434, July-December (1984) 



\section{Danksagung}

Diese Arbeit wurde finanziell im Rahmen eines Stipendiums von der Deutschen Forschungsgemeinschaft im Graduiertenkolleg

„Nichtlinearität und Nichtgleichgewicht in kondensierter Materie“

unterstützt, der ich hiermit dafür danken möchte.

Alle Personen aufzulisten, die diese Arbeit ermöglicht haben, würde wohl den Rahmen dieser Arbeit (endgültig) sprengen. Von daher werde ich mich auf die drei folgenden Personenkreise beschränken.

Der erste Personenkreis setzt sich aus meiner alten Arbeitsgruppe am Lehrstuhl Kalbitzer unter Leitung meines Doktorvaters Elmar Lang und Hauptverantwortlichen, dass diese Arbeit als solches generell existiert, zusammen. Auf Grund meiner langen Halbwertszeit in dieser Arbeitsgruppe gibt es zu viele Personen, als das alle aufgelistet werden können. Bedanken möchte ich mich aber trotzdem bei Daniela Herold, Peter Gruber, Harold Gutch, Dominik Senninger und Angela Zeiler, die direkt und indirekt für ein angenehmes Arbeitsklima gesorgt haben. Explizit bei Angela, zum einen dafür, dass sie es sich mehrfach angetan hat, diese Arbeit Korrektur zu lesen und zum anderen für ihre hilf- und zahlreichen Verbesserungsvorschläge.

Im zweiten Personenkreis finden sich Herr Professor Brawanski, Direktor der Neurochirurgie am Universitätsklinikum in Regensburg, und Rupert Faltermeier. Die Zusammenarbeit kann nur als hervorragend bezeichnet werden. Explizit möchte ich Rupert danken, der diese Arbeit mehrfach Korrektur gelesen hat, aber bereits von Anfang an diese Arbeit mitbetreut und durch seine Vorschläge und Anmerkung die Qualität dieser deutlich verbessert hat.

Zum Schluss möchte ich mich auch noch bei meinen Kollegen und Kolleginnen aus dem Rechenzentrum bedanken, die es mir ermöglicht haben, trotz einer anstrengenden Woche doch noch etwas Zeit zu finden, diese Arbeit fertigzustellen. Zusätzlich möchte ich mich noch zum einen bei meinem Abteilungsleiter Josef Spangler und dem Rechenzentrumsleiter Martin Wimmer bedanken, die es mir durch mehrmalige Anpassung mein Arbeitszeit ermöglichten, neben Urlaub die notwendige Zeit am Stück zu finden, größere Teile in der Arbeit vorwärts und letztendliche diese Arbeit zu Ende zu bringen. Zum anderen möchte ich natürlich noch den aktiven Teilnehmern der 
tägliche Kaffeerunde danken, für ein angenehmes Arbeitsklima und interessante Diskussionen. 